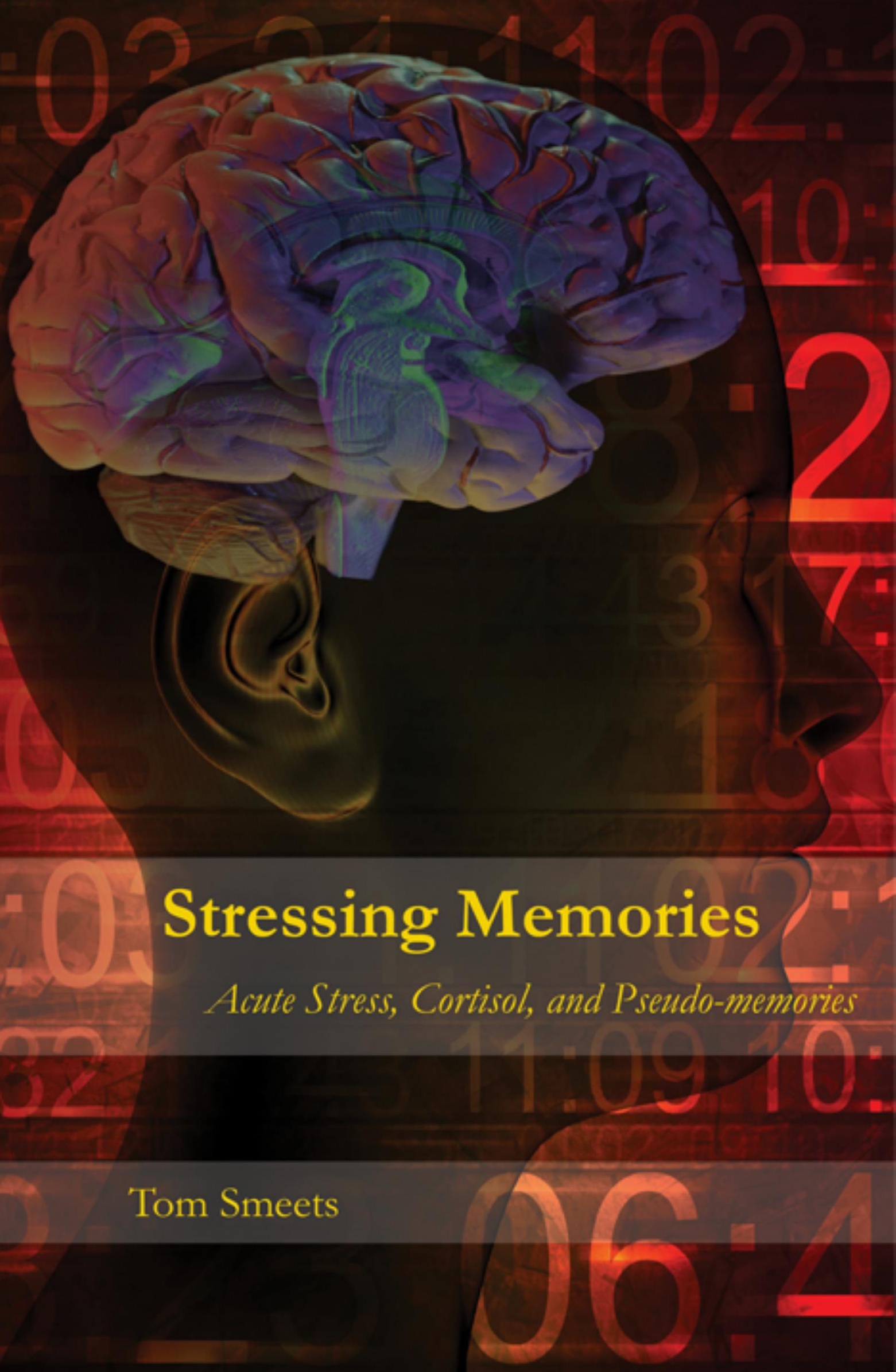




\section{STRESSING MEMORIES}

Acute Stress, Cortisol, and Pseudo-memories 
ISBN: 978-90-5278-590-5

Universitaire Pers Maastricht

Copyright @ ( Tom Smeets, Maastricht 2007 


\section{STRESSING MEMORIES}

Acute Stress, Cortisol, and Pseudo-memories

\section{PROEFSCHRIFT}

ter verkrijging van de graad van doctor aan de Universiteit Maastricht, op gezag van de Rector Magnificus, prof. mr. G.P.M.F. Mols volgens het besluit van het College van Decanen, in het openbaar te verdedigen op donderdag 25 januari 2007 om 14.00 uur

door

Tom Smeets

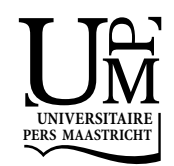




\section{Promotor:}

Prof. dr. H.L.G.J. Merckelbach

\section{Copromotor:}

Dr. M. Jelicic

\section{Beoordelingscommissie:}

Prof. dr. J. Jolles (voorzitter)

Prof. dr. A.R. Arntz

Prof. dr. L.J.P. van Doornen (Universiteit Utrecht)

Dr. B.M. Elzinga (Universiteit Leiden)

Dr. N.A. Nicolson

The research presented in this dissertation was supported by the Netherlands Organization for Scientific Research (NWO, The Hague) grant number 452-02-006 awarded to dr. M. Jelicic. 


\section{Contents}

Chapter 1: Some general remarks on stress, glucocorticoids, memory processes, and pseudo-memories 9

A history of "stress". 10

The stress response 10

Does stress damage the brain? 13

Acute stressors and their cortisol responses 15

Acute stress, glucocorticoid receptor types, and memory 17

Variables modulating the memory effects of acute stress and glucocorticoids 18

Sex differences 21

Cortisol, valence, and emotional arousal 21

Stress and pseudo-memories $\quad 23$

Concluding remarks $\quad 25$

$\begin{array}{ll}\text { Chapter 2: Dissertation outline } & 27\end{array}$

A typical case: The case of A.M.N. 28

Outline of the present dissertation $\quad 28$

Stress, cortisol, and (pseudo-)memory 29

Opinions and limitations in pseudo-memory research $\quad 30$

Chapter 3: The effect of acute stress on memory depends on word valence

Summary 34

Introduction $\quad 34$

Methods $\quad 37$

Results $\quad 41$

Discussion $\quad 44$ 
Chapter 4: Stress-induced cortisol responses, sex differences, and false recollections in a DRM paradigm

Summary

Introduction

STUDY 1

Methods

Results

Discussion

STUDY 2

Methods

Results

Discussion

General Discussion

Chapter 5: Enhanced memory performance on an internalinternal source monitoring task following acute psychosocial stress

Summary

Introduction

Methods

Results

Discussion

Chapter 6: Delayed recall of childhood sexual abuse memories and the awakening rise and diurnal pattern of cortisol

Summary

Introduction

Methods

Results

83

Discussion 
Chapter 7: Trying to recollect past events: Confidence, beliefs, and memories

Summary

Introduction

Neurotransmitters as a metaphor for false memories

Imagination

Suggestion

The El-Al crash

The story so far and its significance

Confidence estimates, false beliefs, and false memories 101

Models

Behavioural sequence

Anxiety research

A look at future research

Chapter 8: "Of course I remember seeing that film!"-How ambiguous questions generate crashing memories

Summary

Introduction

Methods

Results

Discussion

Introduction

Stressing memories: Stress and cortisol as determinants of false recollections?

Summary of research findings

Contradictions and limitations

Reconstruction: Adaptive or maladaptive?

An empirical interlude

State- and mood-state dependent effects

A glance at future research

Concluding remarks 
Works cited

Summary

Samenvatting

Dankwoord

Curriculum vitae 183

List of publications 185 


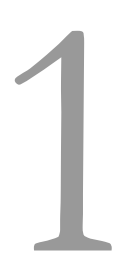

\section{Some general remarks on stress, glucocorticoids, memory processes, and pseudo-memories}




\section{A history of "stress" 1}

19th Century French physiologist Claude Bernard (1865/1961) advanced the idea that one's ability to regulate the milieu interieur (i.e., the internal environment) is critical to life. In order to survive, the internal environment of the body has to be controlled within narrow limits to provide individual cells with enough nutrition and opportunity to remove waste products (Lovallo \& Thomas, 2000). This way, humans could go through life in a steady, constant state called the dynamic equilibrium. Hence, environmental changes or external forces that are capable of changing the dynamic equilibrium must be reacted to and compensated for if the organism has any intention of surviving.

Walter Cannon (1929), a prominent neurologist and first chair in Physiology at Harvard University, coined the term "homeostasis" to refer to the regulation of the dynamic equilibrium and to further define the processes Bernard had described. In his groundbreaking experiments, Cannon demonstrated that humans and animals share a certain "fight or flight" response when confronted with a threatening situation. Here, the internal environment adapts itself to meet demands. Cannon employed the term "stress" to refer to these physiological and psychological demands imposed on organisms and their subsequent attempts to return to "homeostasis". ${ }^{2}$ He was also the first to recognize that stressors could be emotional as well as physical.

Though Cannon was the first author to use the term stress, it was Hans Selye who further popularized the concept of stress through a pioneering series of studies on severe stress in rats (Selye, 1936). In these experiments, rats that were exposed to chronic stress were found to respond with distinctive and stable psychophysiological changes he called the general adaptation or stress syndrome. Although he initially labelled these reactions adaptive (i.e., healthy, normal adjustment), Selye (e.g., 1956) later discovered that these processes, when excessive, could actually damage the body (e.g., adrenal glands enlargement, gastrointestinal ulcers). It is through these initial observations that the concept of stress has acquired such a negative connotation.

\section{The stress response}

Seyle (e.g., 1956) was also the first to describe the stress response. According to Selye, the stress response could be attributed to hypothalamic-pituitary-adrenal

\footnotetext{
${ }^{1}$ For a more elaborate review on how the concept of stress initially developed, see Selye (1973).

2 Man's ability to physiologically respond to threat and to re-establish homeostasis was later called “allostasis" by Sterling and Eyer (1988). Even later, McEwen (2002) added to this definition the concept of a "set point" that varies to maintain a stable internal environment.
} 
(HPA) axis functioning. The HPA axis is responsible for the release of hormones in response to both psychological and physical stressors. This is why we breathe heavily and our perspiration increases, our pupils are dilated, we feel our heart racing, and are ready to react to confrontations with stressors. When we experience the stressor as something positive (e.g., the kick of speed when driving a race car), the adrenaline rush is regarded as positive and exciting. On the other hand, in case the stressor is perceived as something bad or dangerous (e.g., a parachute that will not open in mid air), the appraisal of the stressor will likely to be negative (at best).

However, in emphasizing the role of the HPA axis, Selye largely ignored sympathetic nervous system contributions to the stress response, including the activation of the adrenal medulla that triggers the release of adrenaline (or epinephrine) and noradrenaline (or norepinephrine). Most contemporary theories now acknowledge the importance of both systems in the stress response (e.g., Lovallo, 2005; Stanford \& Salmon, 1993). These two components of the endocrine stress system share considerable central anatomic interconnection. The acute response to stress, usually lasting a few minutes, comprises sympathoadrenal medullary (SAM) activation. The chronic stress response, on the other hand, is mediated by the HPA axis. Although the SAM component is highly relevant to the stress response, the following paragraphs will be mainly restricted to the functioning of the HPA axis. ${ }^{3}$

A typical stress response to a dangerous situation is as follows (see Figure 1.1 for a schematic depiction). First, the sympathetic part of the autonomic nervous system is activated while the parasympathetic branch is simultaneously suppressed. In reaction to the activation, neurons in the sympathetic nervous system trigger the secretion of noradrenaline throughout the body. As a result, the entire body prepares itself for the fight or flight reaction by increasing blood flow to the muscles, increasing heart rate, and rising blood pressure. Then, merely a few seconds later, the adrenal glands release adrenaline serving to further activate the body. In the meantime, corticotropin releasing factor (CRF) is secreted by the hypothalamus in a closed circuit in which the hypothalamus and the anterior pituitary gland play a role. About 15 seconds later, CRF sets off the release of adrenocorticotropic hormone (ACTH) by the pituitary gland. Subsequently, ACTH released within the circulatory system moves via the bloodstream to the adrenal glands which, within minutes, secrete glucocorticoids (GCs; i.e., cortisol in humans and monkeys; corticosterone in rodents) into the bloodstream. These GCs together with the sympathetic nervous system and glucagons released by the pancreas, are then responsible for raising the glucose levels in the blood, in turn producing more energy for the body to use in its reaction to the stressor. Finally, the brain and pituitary gland emit endorphins and enkephalins which serve to diminish pain perception should the

\footnotetext{
${ }^{3}$ For a complete review on neuroendocrine factors mediating the stress response other than those related to the HPA axis (e.g., vasopressin, serotonin, catecholamines), see Charney (2004) and Carrasco and Van de Kar (2003).
} 
organism be harmed. A negative feedback system in which cortisol, a lipophilic steroid, returns to the pituitary and hypothalamus in order to inhibit additional secretion of CRF and ACTH prevents the HPA axis from overshooting. It also helps the system to terminate the stress-induced HPA response and restore the HPA status to normal.

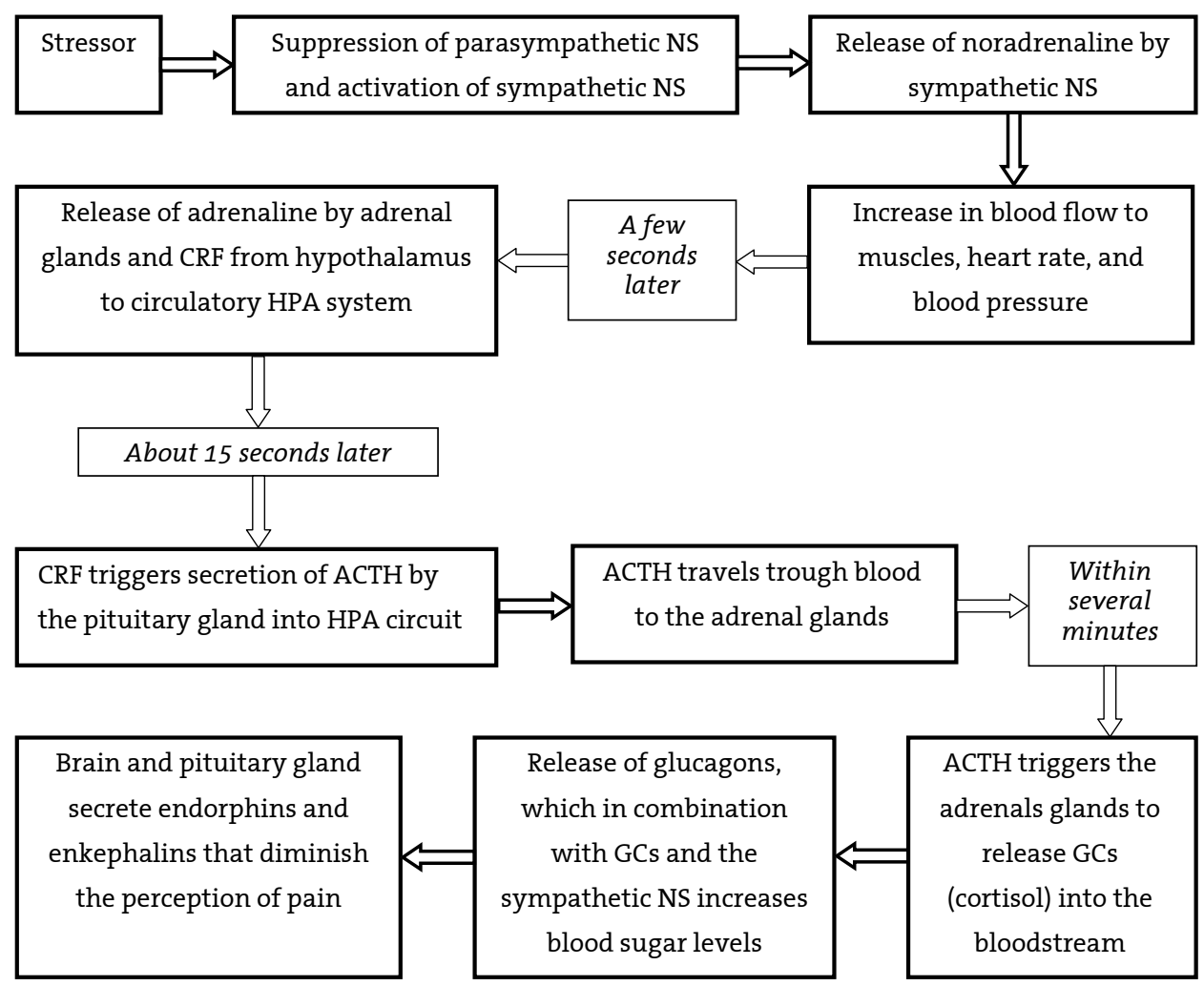

Figure 1.1. Regulation of cortisol secretion during a typical stress response. Notes: NS $=$ nervous system; $\mathrm{CRF}=$ corticotropin releasing factor; HPA = hypothalamic-pituitary-adrenal; ACTH = adrenocorticotropic hormone; GCs = glucocorticoids.

Following secretion, about 90 percent of the total cortisol in the blood binds to the proteins corticosteroid-binding globulin $(\mathrm{CBG})$ and albumin (Kirschbaum \& Hellhammer, 1989). Only 5 to 10 percent of the total cortisol in the blood remains unbound. This percentage of "free", unbound cortisol is the active part in the target cells. Cortisol concentrations at any given time can easily be measured via saliva sampling procedures, and these measurements have been shown to be a valid and reliable reflection of the free unbound concentrations in the blood (e.g., Kir- 
schbaum \& Hellhammer, 1989; 1994). The unbound, biologically active cortisol readily passes the blood-brain barrier and, as such, is capable of reaching the central nervous system, and enters the cerebrospinal fluid to reach regulatory sites in the brain such as the amygdala or the hippocampus (Lovallo \& Thomas, 2000).

Cortisol is generally thought to have at least 2 different modes of activation. In one state, the permissive state, cortisol serves normal metabolic (e.g., replenishing energy stores) and diurnal functions. These functions are driven by the input from the hypothalamus and are highly sensitive to negative feedback control. In its second state, high levels of cortisol have regulatory functions during periods of stress by exercising regulatory control over stress-related processes (Lovallo \& Thomas, 2000). Here, cortisol secretion is promoted by signals from the amygdala and negative feedback control is much less effective. Along these lines, one may conclude that cortisol allows for optimal adaptation to the ever changing demands from the environment. ${ }^{4}$

\section{Does stress damage the brain?}

Although clearly adaptive in the short run, various authors have speculated about the effect that the stress response may have over prolonged periods of time. Indeed, there have been reports purporting to show that intense stress responses can cause various diseases or conditions, including anxiety disorders, a variety of ulcers and other gastrointestinal diseases, and even damage to certain brain structures (e.g., hippocampus) that eventually may result in memory deficits. It are the latter findings describing hippocampal and other structural brain damage following chronic stress responses that have attracted most attention of researchers as well as psychotherapists in the trauma field. But can our normal, adaptive response to stress really inflict harm to our brain? If so, what mechanisms are responsible for the putative damage?

Robert Sapolsky, a leading scientist in neurobiology, was amongst the first researchers to suggest that the stress response may actually damage certain areas of the brain, in particular the hippocampus, a medial temporal lobe structure vital to new learning and memory (e.g., Squire, 1992; Zola-Morgan \& Squire, 1990). According to Sapolsky (1996), exposure to high levels of GCs can result in impaired hippocampal functioning and even neuronal cell loss. As the hippocampus is critically important for the regulation of negative feedback of the HPA axis, this loss of hippocampal cells may leave the system less able to perform its regulatory (diurnal and metabolic) function of cortisol secretion. This in turn would cause even higher levels of cortisol, resulting in chronically elevated GC levels and ultimately render-

\footnotetext{
${ }^{4}$ Next to the permissive and regulatory activities, glucocorticoids have also been implicated in stimulatory and preparative actions (e.g., Sapolsky, Romero, \& Munck, 2000).
} 
ing the hippocampus extra vulnerable to hippocampal atrophy, and so on. This hypothesis is known as the "glucocorticoid cascade" or, alternatively, the "stressinduced glucocorticoid toxicity" hypothesis (e.g., Bremner, 1999, 2002; O’Brien, 1997; Sapolsky, 1996; but see Yehuda, 1997, and Jelicic \& Bonke, 2001, for thoughtful critiques). The importance of the glucocorticoid cascade hypothesis is evidenced by the fact that some authors (e.g., Howe, 1998; Bremner, 2001) have argued that biological factors such as stress-sensitive neuroendocrine systems may cause the loss and perhaps even the recovery of traumatic memories (i.e., recovered memories). These authors argue that childhood abuse results in permanent effects on brain systems and circuits that mediate the stress response. Specifically, Bremner (e.g., 2003, p. 272) maintains that "[...] early adverse experience permanently affects the HPA axis." 5

The glucocorticoid cascade hypothesis is consistent with several observations. Animal research, for instance, has shown that excessive GC blood levels can be damaging to the hippocampus of rodents and monkeys (e.g., Sapolsky, Krey, \& McEwen, 1985; Uno, Tarara, Else, Suleman, \& Sapolsky, 1989; Watanabe, Gould, \& McEwen, 1992; Woolley, Gould, \& McEwen, 1990). More specifically, the hippocampus of rats and monkeys injected with GCs shows increased vulnerability to all kinds of neurological insults such as hypoglycaemia and oxygen deficiencies. Also, under the influence of raised GC levels, dendrites (i.e., neuronal branches that receive signals from other neurons) may begin to atrophy (e.g., Sapolsky \& Pulsinelli, 1985; Woolley et al., 1990). In addition to animal work, it is widely known that patients with Cushing's disease, an endocrinological disorder characterized by prolonged excessive secretion of cortisol (caused by, e.g., a corticotroph adenoma or an ectopic tumour; see Nieman \& Cutler, 1995) show reduced hippocampal volume. Moreover, patients suffering from Cushing's disease suffer from explicit declarative memory deficits, as evidenced by landmark studies of Monica Starkman and co-workers (e.g., Starkman \& Schteingart, 1981; Starkman, Giordani, Berent, Schork, \& Schteingart, 2001; see also Mauri et al., 1993).

It should be noted, however, that surgical treatment of Cushing's disease leads to a rapid normalization of cortisol levels. More importantly, memory impairments also disappear and hippocampal volume normalizes (Starkman, Giordani, Gebarski, Berent, Schork, \& Schteingart, 1999; Starkman, Giordani, Gebarski, \& Schteingart, 2003). Then again, there are numerous other observations that strike at the heart of the glucocorticoid cascade hypothesis. Recall that this

\footnotetext{
5 The impact of this hypothesis is also illustrated by the fact that some expert witnesses (e.g., Bremner) have used in courts of law Magnetic Resonance Imaging (MRI) scans showing complainants' reduced hippocampal volume in comparison to their peers so as to persuade juries that these patients had experienced traumatic childhood events. The experts argue that this "evidence of hippocampal atrophy" can explain why these individuals at first were amnesic for their traumatic childhood events and why they, with the help of psychotherapeutic treatment, have recovered traumatic memories and have now taken matters to court (e.g., Bremner, 2002).
} 
hypothesis implies that (traumatic) stressors may deregulate the HPA axis over an extended time interval and elevate cortisol levels so high that irrevocable hippocampal damage occurs and memory deficits become apparent. However, the seminal studies on patient H.M. (Scoville \& Milner, 1957) illustrate that hippocampal damage prevents the encoding of new memories. Thus, even if chronically elevated cortisol levels would result in hippocampal damage, this still would not explain any memory impairments for old material (i.e., memories that were consolidated prior to the stressful experiences, such as the onset of a traumatic experience). Furthermore, the largest part of research claiming to show clear relationships between stress, brain alterations, and subsequent memory deficits, suffers from methodological problems that gravely limit its validity (Jelicic \& Bonke, 2001; Jelicic \& Merckelbach, 2004). For example, most studies have used a cross-sectional design, making it difficult to draw causal inferences. Finally, also problematic to the glucocorticoid cascade hypothesis is the fact that patients suffering from post-traumatic stress disorder (PTSD) following trauma exposure do not tend to exhibit higher levels of GCs, but instead generally show lowered cortisol levels (for a review on PTSD and its biological aspects, see Yehuda, 2002).

Perhaps, then, it is the primary release of cortisol in direct response to a traumatic stressor that is so extremely immense that hippocampal damage almost immediately occurs. This scenario is unlikely for two reasons (McNally, 2003). Firstly, Sapolsky's work on animals (cf. supra) shows us that it takes at least a few weeks of exposure to exceptionally high cortisol levels in order for hippocampal damage to occur. Secondly, cortisol secretion is an adaptive component of the stress response. Sudden cortisol elevations of extreme magnitude clearly are anything but adaptive. Therefore, the cortisol secretory system cannot be seen as an active mechanism supportive of the glucocorticoid cascade hypothesis. Or, in the words of McNally (2003, p. 143): "A brief, single burst, however massive, will not do any lasting damage." Nevertheless, during the last century, research has gradually become interested in the effects of acute stress and subsequent cortisol responses on memory encoding, consolidation, and retrieval processes. This type of research is not directed at the possibility of brain damage due to stress exposure, but concentrates on milder forms of acute memory impairment. A brief overview of the current knowledge brought forth by this field of research follows in the sections below.

\section{Acute stressors and their cortisol responses}

Despite being somewhat restricted by the ethical issues inherent in human research, there have been increasingly more experimental studies investigating the relationship between acute stress and memory performance. These studies sometimes use naturalistic high risk tasks such as skydiving (e.g., Cavenett \& Nixon, 
2006; Thompson, Williams, L'Esperance, \& Cornelius, 2001) or intense military training (e.g., Lieberman, Bathalon, Falco, Kramer, Morgan, \& Niro, 2005; Morgan et al., 2004), but most often they generate stress by exposing research participants to single, short laboratory stressors. Examples of these obviously less dangerous acute psychosocial stressors include public speaking tasks, mental arithmetic tasks, and noise exposure (for a review on acute laboratory stressors and their GC effects, see Dickerson \& Kemeny, 2004). The most frequently used laboratory psychosocial stressor is the Trier Social Stress Test (TSST; Kirschbaum, Pirke, \& Hellhammer, 1993). This challenge test basically consists of a 10 minute preparation period, a 5 minute free speech, and a 5 minute mental arithmetic task in front of an audience while being videotaped. The TSST is widely know as a valid and reliable procedure to induce physiological stress responses in children and young as well as elderly adults (e.g., Kirschbaum, Wüst, \& Hellhammer, 1992; Kudielka, Buske-Kirschbaum, Hellhammer, \& Kirschbaum, 2004a; Kudielka, Schommer, Hellhammer, \& Kirschbaum, 2004b).

It is worthy of note here that research indicates that HPA axis (i.e., cortisol) stress responses do not parallel subjective stress responses to psychological stressors (e.g., Buske-Kirschbaum, von Auer, Krieger, Weis, Rauh, \& Hellhammer, 2003; Frankenhaeuser, Dunne, \& Lundberg, 1976; Kirschbaum et al., 1996a; Kudielka et al., 1998). This highlights the importance for not relying on verbal, written, or other subjective reports of perceived stress, but that measuring cortisol responses is essential for gauging the physiological stress reaction. Additionally, one should bear in mind that several factors may modulate the GC response to acute psychosocial stressors such as the TSST (for thorough reviews, see Kajantie \& Philips, 2006; Kudielka \& Kirschbaum, 2005; Otte, Hart, Neylan, Marmar, Yaffe, \& Mohr, 2005). Probably the most often reported and well-studied variability in cortisol responsiveness concern sex differences. ${ }^{6}$ In a series of studies, Clemens Kirschbaum and his colleagues (Kirschbaum et al., 1992) used the TSST to investigate differences in stress responsiveness between healthy men and women. These authors consistently found that men produced the strongest cortisol responses, often 1.5 to 2 fold of what is typically found in women. However, no sex differences were observed in a handful of other studies that looked at infants' adrenocortical responses (e.g., Benjamins, Asscheman, \& Schuurs, 1992; Larson, Gunnar, \& Hertsgaard, 1991; Lewis \& Thomas, 1990). This led Kudielka and Kirschbaum (2005, p. 117) to conclude that " $[. .$.$] most psychological stress studies revealed that there are (a)$ no significant sex differences or (b) higher cortisol responses in young men than in young women $[\ldots]$ ".

Closely related to sex differences are reports on differential stress responses for women using oral contraceptives and women who are in various stages of their

\footnotetext{
${ }^{6}$ Because the stress-cortisol-memory topic of this dissertation is rather biological in nature, we prefer to use the term "sex" instead of "gender".
} 
menstrual cycle (e.g., Kajantie \& Philips, 2006). It seems that women who use oral contraceptives tend to show the smallest adrenocortical responses to psychosocial challenges, while women in the luteal phase (i.e., days 21-25 of the menstrual cycle) show cortisol responses that are similar to those of stressed men (e.g., Kirschbaum, Kudielka, Gaab, Schommer, \& Hellhammer, 1999). Data on cortisol responses in pregnant women, however, have yielded mixed results (Kirschbaum \& Hellhammer, 1989). In addition to these sex-related differences in cortisol responses, the use of any kind of medication or nicotine usage may profoundly affect participants' HPA axis responses. Therefore, when measuring cortisol, drug intake should be restricted or, even better, avoided at all times. Finally, a number of studies have suggested that individual differences in cortisol reactivity may very well reflect genetic predispositions (e.g., Wüst, van Rossum, Federenko, Koper, Kumsta, \& Hellhammer, 2004), anticipatory cognitive appraisal (e.g., Gaab, Rohleder, Nater, \& Ehlert, 2005; Rohrmann, Hennig, \& Netter, 1999), or polymorphism in sensitivity of GC receptor tissue (e.g., Rohleder, Wolf, \& Kirschbaum, 2003).

\section{Acute stress, glucocorticoid receptor types, and memory}

As to their consequences for memory processes, acute stress-induced GC memory effects are determined by the activation of mineralocorticoid (MR or type-I) and glucocorticoid (GR or type-II) receptors (e.g., de Kloet, Oitzl, \& Joëls, 1999). While mineralocorticoid receptors bind to GCs as well as aldosterone, GRs have binding domains that are specific to GCs. The high affinity mineralocorticoid receptors are almost exclusively localized within the limbic system (e.g., various frontal regions, amygdala, hippocampus). They are mainly occupied in normal conditions, determine the basal HPA axis regulation, and control the sensitivity of the CRF driven stress system. The low affinity GC receptors, on the other hand, are not restricted to the limbic system. They are widely distributed throughout the entire brain, with dense expression in stress centres such as the hippocampus, paraventricular nucleus, and pituitary corticotropes. It is only when cortisol levels rise that GC receptors become exceedingly saturated and give an inhibitory signal to the pituitary gland to reduce the ACTH production. Thus, while GCs act on both types of receptors, they encompass differential effects.

It appears that when high affinity mineralocorticoid receptors are fully occupied while GC receptors are only partially activated, memory can be facilitated. Alternatively, detrimental effects of high GC levels may occur when GC receptors become exceedingly saturated during stressful situations (e.g., de Kloet et al., 1999; Oitzl \& de Kloet, 1992; Reul \& de Kloet, 1985). Also, adrenergic activation in the basolateral complex of the amygdala (BLA) and in the hippocampus seems to be required for GCs to impair retrieval (Roozendaal, Hahn, Nathan, de Quervain, \& McGaugh, 2004). Furthermore, activation of noradrenergic mechanisms in the 
BLA may, in combination with several other brain regions including the hippocampus and prefrontal cortex (PFC), mediate the effects of emotional arousal in combination with GC effects on memory functioning (e.g., McGaugh \& Roozendaal, 2002).

\section{Variables modulating the memory effects of acute stress and glucocorticoids}

A vast amount of animal research has revealed that the secretion of GCs may modulate the encoding and consolidation of memories (e.g., de Kloet et al. 1999; McGaugh, 2000; Roozendaal, 2000). In animals, GCs can have facilitating (e.g., on aversive conditioning) as well as impairing effects on memory (e.g., de Kloet et al., 1999; Lupien \& McEwen, 1997; McGaugh \& Roozendaal, 2002). Studies involving human participants demonstrate similar findings in that acute GC administration may enhance or disrupt memory, depending on a host of modulatory variables (for reviews, see Het, Ramlow, \& Wolf, 2005; Lupien et al., 2005; Lupien \& Lepage, 2001; Wolf, 2003). One of the most influential variables is the timing of GC administration or stress exposure. It seems that when cortisol (or an acute stressor for that matter) is given to participants before the retrieval phase of the experiment, there is a significant decrease in memory performance (de Quervain, Roozendaal, Nitsch, McGaugh, \& Hock, 2000; Wolf, Kuhlmann, Buss, Hellhammer, \& Kirschbaum, 2004). De Quervain and co-workers (2000), for example, gave their participants synthetic GCs (cortisone) 1 hour before learning a word list, 1 hour after learning it, or before the recall test that followed 24 hours after learning the list. These authors found that GC administration impaired delayed recall performance, but only so for the group that received GCs before retrieval. Although there have been studies reporting slightly enhancing effects of GCs on memory formation (e.g., Buchanan \& Lovallo, 2001), studies in which stress exposure or GC administration was accomplished before learning have generally yielded nonsignificant results (Het et al., 2005).

Another important modulator that deserves to be mentioned here is the time of day when GCs are administered or stress is applied to participants. In particular, it seems that when GCs are given in the morning hours they yield detrimental memory effects, while GCs given in the afternoon either have no effect or exert a small enhancing effect on memory. A good example of work substantiating these conclusions comes from a recent study by Maheu, Collicutt, Kornik, Moszkowski, and Lupien (2005a). These authors had 19 young men watch a story after being subjected to a psychological stress task (i.e., stress group), while another 20 men did so without being exposed to a stressor (i.e., controls). The crux of the matter is that Maheu et al. further divided both groups in either a morning group that saw 
the memory material in the morning (i.e., at $09.40 \mathrm{~h}$ ) or an afternoon group that saw it at $14.40 \mathrm{~h}$ (i.e., the afternoon group). When tested for their memory one week later, those participants who were stressed and had viewed the material in the morning, as compared to the afternoon stress group, showed impaired recall performance for emotional details of the memory material.

As to what type of memory (i.e., explicit or implicit) is affected by stressinduced GC secretion, prior research revealed that for humans the effects may be detrimental to (explicit) declarative memory performance, while leaving implicit memory (i.e., memory retrieval in which an individual shows enhanced performance on a memory task as a result of prior experience, despite having no conscious awareness of recollecting the prior experience; e.g., priming effects) intact (e.g., Kirschbaum, Wolf, May, Wippich, \& Hellhammer, 1996b; Newcomer, Craft, Hershey, Askins, \& Bardgett, 1994; Newcomer et al., 1999). Kirschbaum and colleagues (1996b), for example, exposed healthy volunteers to an acute psychosocial stressor. On a subsequent declarative memory test, Kirschbaum et al. (1996b, Experiment 1) found that participants with stronger cortisol responses to the stressor recalled fewer words than participants showing only a minor cortisol response. In their second experiment, Kirschbaum et al. (1996b) noted that participants who had received cortisol, performed less well on a declarative memory task than participants who had received a placebo. Similarly, a study by Newcomer and colleagues (1999) showed that the administration of cortisol over several days led to impaired performance on a verbal memory task in healthy volunteers. Over the past decade, many studies (e.g., Abercrombie, Kalin, Thurow, Rosenkranz, \& Davidson, 2003; de Quervain et al., 2000; Domes, Rothfischer, Reichwald, \& Hautzinger, 2005; Kuhlmann, Kirschbaum, \& Wolf, 2005a; Lupien et al., 1997) have replicated and extended these findings showing the detrimental effects of acute stress and/or cortisol elevations on declarative memory performance. Table 1.1 presents a summary of typical studies in the field. 


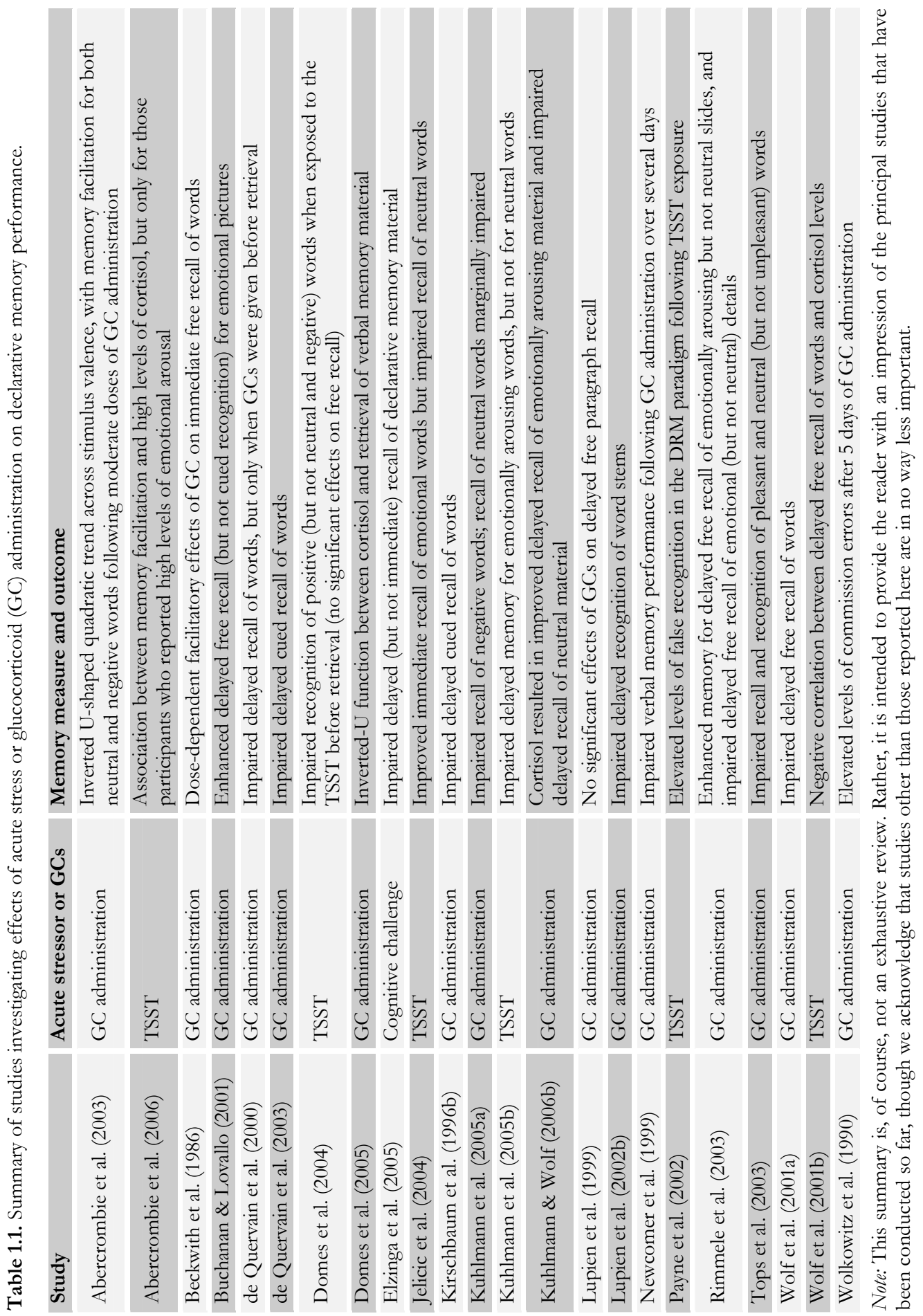




\section{Sex differences}

To complicate matters even further, there are indications suggesting that effects of stress hormones (e.g., cortisol) on memory functioning may be different for men and women. These indications come from animal (e.g., Conrad et al., 2004; Luine, 2002; Wood, Beylin, \& Shors, 2001; Wood \& Shors, 1998) as well as human (e.g., Seeman, McEwen, Singer, Albert, \& Rowe, 1997; Wolf, Kudielka, Hellhammer, Hellhammer, \& Kirschbaum, 1998; Wolf, Schommer, Hellhammer, McEwen, \& Kirschbaum, 2001b; for review, see Wolf, 2003) studies. For example, Seeman and colleagues (Seeman et al., 1997) found that higher basal levels of cortisol were associated with impaired memory performance among elderly women, but not among men. In line with these results, Wolf and colleagues (Wolf et al., 1998) noted that exposure to the TSST following Dehydroepiandrosterone (DHEA) treatment significantly impaired memory performance in elderly women, but not in elderly men. In a follow-up study, Wolf and co-workers (Wolf et al., 2001b) found that within a group of young adults exposed to the TSST, cortisol increases were negatively correlated with memory performance. Further analysis, however, revealed that this effect was carried by a strong negative correlation in men while no such correlation was found among women.

\section{Cortisol, valence, and emotional arousal}

The effect of cortisol on memory performance also depends on the valence and emotional arousal of the material being studied. For example, Tops et al. (2003) showed that 2 hours after participants were given either $10 \mathrm{mg}$ cortisol or placebo, those who had been given cortisol exhibited impaired recall and recognition of neutral and pleasant words, whereas no difference was found between both groups for unpleasant words. Using a more natural way to elicit high levels of cortisol, Jelicic, Geraerts, Merckelbach, and Guerrieri (2004) exposed half of their participants to a TSST, while the other half was given a non-stressful control task. They found that relative to controls, participants in the stress condition exhibited enhanced recall of emotional words, but showed impaired recall of neutral words. In a study by Abercrombie et al. (2003), 90 men were administered either $20 \mathrm{mg}$ or $40 \mathrm{mg}$ of hydrocortisone or placebo and were later asked to rate a list of negative and neutral words for pleasantness and arousal. At a follow-up session 2 days later, Abercrombie et al. found evidence for an inverted U-shaped quadratic trend across stimulus valence, with memory facilitation for both neutral and negative words in the $20 \mathrm{mg}$ hydrocortisone group. These results suggest that only moderate doses of hydrocortisone enhance memory for words, irrespective of the valence of the studied material. 
Confirming these results, Rimmele and co-workers (Rimmele, Domes, Mathiak, \& Hautzinger, 2003) had their participants view an emotionally arousing or a neutral story following the administration of $25 \mathrm{mg}$ of hydrocortisone or a placebo. One week later, participants who had been given cortisol exhibited enhanced memory for details of the neutral story version, but impaired memory for details of the emotional story. In Domes, Heinrichs, Rimmele, Reichwald, and Hautzinger's (2004) study, participants learned a word list containing neutral, pleasant, and unpleasant words either before or after they had been exposed to the TSST, or had not been stressed at all. While free recall was not affected by exposure to the TSST, recognition memory for positive words was impaired for participants who were stressed before retrieval. In a study by Kuhlmann, Piel, and Wolf (2005b), healthy young men who had learned a word list including neutral, negative, and positive words 24 hours earlier were either assigned to a stress (TSST) or a control condition. In a subsequent free recall task, participants in the stress condition showed impaired memory for the emotionally arousing, but not the neutral words. In yet another study, Kuhlmann, Kirschbaum, and Wolf (2005a) exposed 16 healthy female participants to either $30 \mathrm{mg}$ hydrocortisone or placebo after they had learned a list containing neutral and negative words. Kuhlmann et al. found that recall of negative words was most strongly impaired at a recall test 5 hours later, while recall of neutral words only tended to be modestly impaired.

Finally, related to the interaction between stimulus valence and cortisol effects is the emotional arousal elicited by the memory material. Cases in point are recent studies by Abercrombie, Speck, and Monticelli (2006) and Kuhlman and Wolf (2006b). In Abercrombie and co-workers' (2006) study, participants were exposed to a modified TSST after they had been shown emotional and neutral pictures. Furthermore, these authors assessed participants' emotional arousal in terms of self-reported negative affect at baseline and after stress exposure. Abercrombie et al. found that high cortisol responses were associated with memory facilitation, but that this beneficial effect of cortisol was restricted to those participants who reported high levels of emotional arousal. Following a similar methodology, Kuhlman and Wolf (2006b) recently gave their participants $30 \mathrm{mg}$ of cortisol or placebo prior to the presentation of emotionally arousing and neutral pictures. In line with the results of Abercrombie et al. (2006), participants who were administered cortisol exhibited improved memory for emotionally arousing and impaired memory for neutral material at a delayed recall test 24 hours later. Thus, it seems that not only valence, but also emotional arousal of the to-be-remembered stimuli accounts for some of the contradictory findings in research on the memory effects of cortisol. 


\section{Stress and pseudo-memories ${ }^{7}$}

Given the reconstructive nature of episodic memory, it is not astonishing that individuals may remember events or facts differently from how they originally occurred or may even remember events that did not take place at all. Interest in these so-called pseudo-memories was sparked by a flood of cases in which people in psychotherapy suddenly came to "recover" what seemed to be previously inaccessible memories of traumatic childhood events. The ensuing debate (i.e., the "memory wars") among experimental as well as clinical psychologists and psychotherapists and other clinicians has been one of psychology's most fiercely contested, malicious, and emotionally charged debates. A positive by-product of this debate, however, is that it has led to a massive experimental literature on how certain manipulations may distort memory or even create entire pseudo-memories. One of the most influential paradigms intended to elicit pseudo-memories is the DeeseRoediger-McDermott paradigm (DRM; Deese, 1959; Roediger \& McDermott, 1995). Typically, participants are presented with semantically associated word lists (e.g., thread, sewing, sharp, point, haystack, injection). After presentation of the list(s), participants are instructed to freely recall as many of the words they can remember. Subsequently, a test probing for recognition of presented words (e.g., sharp), non-presented unrelated distracter words (e.g., rain), and a semantically related non-presented word termed the "critical lure" (in this case the word needle) follows. In general, people often falsely recall and/or recognize semantically related but non-presented words (i.e., the critical lures) as having been presented in the list learning task (e.g., Roediger, Jacoby, \& McDermott, 1996; Roediger \& McDermott, 1995; Roediger \& McDermott, 2000).

As reviewed above, acute stress and cortisol can be detrimental to declarative memory performance in that recall as well as recognition of the previously presented memory material may deteriorate. In other words, what acute stress and/or cortisol have been shown to affect is the relative amount of correctly recalled memory material and, thus, the relative amount of memory material that was omitted. However, this is only one aspect of memory. Another index is provided by the number of distortions and commission errors (e.g., Candel, 2003; see also Smeets, Candel, \& Merckelbach, 2004). ${ }^{8}$ A distortion can be defined as a change in the de-

\footnotetext{
7 Throughout the present dissertation, we prefer to use the neutral term pseudo-memory rather than the popular term false memory, which has negative connotations and has led to some terminological confusion (see Chapter 7 of this dissertation). Additionally, when we refer to recollections of nonpresented words in standard list learning paradigms, we will term these extra-list intrusions commissions, and, in the case of the DRM paradigm, false recollections of critical lure words.

${ }^{8}$ Closely related to this issue is Koriat, Goldsmith, and Pansky's (2000) historical point that two main approaches to the study of memory can be distinguished. Firstly, the quantity-oriented approach regards memory performance in terms of the number of to-be-remembered items that is retrieved from memory. Secondly, the accuracy-oriented approach considers memory as a reconstruction of
} 
tails of an existing item (e.g., recalling "anxious" instead of "anxiety"), and a commission error can be described as the introduction of an entirely novel element (Gudjonsson \& Clare, 1995). Since these memory indices reflect relatively independent dimensions (e.g., Smeets et al., 2004), the effects of acute stress (or cortisol) on commission errors and pseudo-memories may be different from those observed on memory tasks evaluating veridical declarative memory performance.

Turning back to Table 1.1, it may surprise the reader to see that virtually all of the studies included in this table have looked at the relative amount of correctly recalled items. Indeed, most studies have not specifically focused on distortions or commission errors. Exceptions to this include the studies by Wolkowitz et al. (1990) and Payne and co-workers (Payne, Nadel, Allen, Thomas, \& Jacobs, 2002). Wolkowitz and colleagues exposed participants to $80 \mathrm{mg} /$ day of prednisone or placebo for 5 consecutive days. Relative to controls, those who had received the GC prednisone generated more commissions in a verbal memory task. In Payne et al.'s (2002) study, participants who had been exposed to the TSST or a non-stress filler task were required to listen to 20 DRM word lists, each followed by a computerized recognition task. Compared to controls, participants exposed to the TSST showed elevated rates of false recognition for non-presented theme words (i.e., critical lures).

On a related note, there have been studies investigating whether people who had been exposed to severely stressful experiences are more prone to false recall and false recognition as elicited by the DRM paradigm. In a study by Zoellner, Foa, Bartholomew, and Przeworski (2000), traumatized individuals (i.e., victims of assault) with and without PTSD were compared to a non-traumatized control group on levels of false recollective experiences. These authors found that relative to non-traumatized controls and irrespective of their PTSD status, traumatized individuals were more prone at falsely recalling the non-presented critical lures. Similarly, Bremner, Shobe, and Kihlstrom (2000) compared women with a history of childhood sexual abuse (CSA) to a non-abused control group and found that abused women with PTSD showed higher levels of false recognition than abused women without PTSD as well as non-abused men and women. Yet another study that employed the DRM paradigm to look at memory functioning in traumatized individuals is a study by Clancy, Schacter, McNally, and Pitman (2000). These authors reported that, relative to women reporting repressed or continuous CSA memories and controls without a history of CSA, women reporting recovered memories of CSA were more prone to falsely recognize the critical lures. Replicating these results, Geraerts and co-workers recently reported that women reporting recovered CSA memories were more prone to develop false recollections in the DRM paradigm than women reporting repressed or continuous memories of CSA

the memory material that was studied. Hence, memory is evaluated in terms of its correspondence with what was learned (i.e., the 'correspondence metaphor'). 
and controls, even when trauma-related DRM lists were used (Geraerts, Smeets, Jelicic, van Heerden, \& Merckelbach, 2005).

One of the first models intended to account for the development of pseudomemories was a principle known as "discrepancy detection" (Tousignant, Hall, \& Loftus, 1986; see also Schooler \& Loftus, 1986). According to this principle, recollections are most vulnerable to distortion if a person does not straight away detect discrepancies between post-hoc misinformation and the originally encoded information. Discrepancy detection is said to be influenced by the strength of the encoded information and the way in which the misinformation was presented. This implies that total or at least partial amnesia is required for the development of pseudo-memories. In an intriguing study, Wolf and co-workers (Wolf, Fujiwara, Luwinski, Kirschbaum, \& Markowitsch, 2005) found that patients with severe global amnesia were characterized by a disrupted morning cortisol response (i.e., the acute increase in cortisol levels following awakening that is typically observed in healthy individuals). To the best of our knowledge, this is the first study to provide preliminary evidence for an association between cortisol and amnesia. Thus, both the Wolf et al. study and studies that employed the DRM paradigm to examine memory functioning in trauma victims (e.g., Bremner et al., 2000; Clancy et al., 2000; Geraerts et al., 2005; Zoellner et al., 2000) highlight the importance for more systematic investigations of whether acute stress and/or (lacking) cortisol elevations may produce pseudo-memories. ${ }^{9}$

\section{Concluding remarks}

Summing up, this chapter has provided the reader with a short history of the stress concept and described the normal human response to stress, consisting of fast, SAM driven activity and long-lasting HPA axis regulated actions. This response helps people to react to threatening situations in a rapid, determined, and, with a bit of luck, shielding way. Even though our stress response is undoubtedly adaptive in the short run, there has been some debate on whether long term activation of the stress system and the HPA axis in particular, may result in certain diseases and brain injuries. Although hippocampal damage due to hypercortisolism has been reported in people suffering from Cushing's disease, this process is reversible. In addition, several authors (e.g., McNally, 2003) who have reviewed the wide range of studies investigating the lasting effects of GCs concluded that permanent injuries to the brain are extremely unlikely, and that damage to certain brain structures caused by acute excessive cortisol secretion is virtually impossible.

\footnotetext{
9 Also worthy of note are reports on altered cortisol awakening responses among patients suffering from PTSD (e.g., Rohleder, Joksimovis, Wolf, \& Kirschbaum, 2004; Wessa, Rohleder, Kirschbaum, \& Flor, 2006).
} 
As to the effects of stress hormones on memory performance, the overview presented is this chapter indicates that the issue is complex and encompasses a wide range of diverse findings. A number of modulating variables were identified, but up until now the precise nature of some of these factors remains unclear. One of the interesting topics in research on the memory effects of acute stress and GCs is the valence of the to-be-remembered information (see Chapter 3). In addition, the Payne et al. (2002) study showed that the effects of acute stress are not confined to veridical memory performance, but that acute stress may also potentiate false recollections (see also Chapter 4). However, in order to draw accurate conclusions on whether acute stress may foster pseudo-memories, more systematic research and replication studies are warranted. Clearly, there are still many research questions that require further study and need to be resolved. 


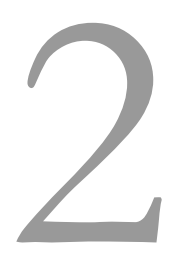

Dissertation outline 


\section{A typical example: The case of A.M.N.}

On a bleak December night, A.M.N., a 23-year old man living in the vicinity of Cologne, Germany, was suddenly alarmed by his discovery of a smouldering fire in the cellar of his house. Upon discovering the fire, A.M.N. immediately ran out of the house and called for help. As he had moved quickly, he did not inhale any smoke and had not touched the fire. Though he was physically unharmed, A.M.N. appeared to be dazed and frightened, and the next morning he no longer remembered who he was or what he did for a living. When told that he worked for an insurance company and that he had a girlfriend for over 3 years now, A.M.N. was unable to remember any details whatsoever and could barely recognize his partner in life.

During the next 2 months of hospital admission, A.M.N. exhibited severe memory impairments, showed flat affect and lack of interest, while his mood was sad and helpless. PET studies performed at the local hospital showed an unusually drastic hypometabolism in memory-sensitive regions. After 3 weeks of psychotherapy, A.M.N. came to remember an early childhood event. He remembered a car crash in which he witnessed the driver's screams and death in flames. The doctors were clear in their conclusion: this is a typical case in which an emotionally negative childhood event (i.e., the car crash he witnessed) and stress re-exposure to a traumatic scene (i.e., the fire in the cellar) led to a state of dissociative amnesia (i.e., an inability to remember).

\section{Outline of the present dissertation}

The case portrayed above is a summary of a case report by Markowitsch and coworkers describing an amnesic patient (for full details on this case vignette, see Markowitsch, Kessler, Weber-Luxenburger, Van der Ven, Alber, \& Heiss, 1998, 2000). It is prototypical in that amnesia, both retrograde and anterograde, is often haphazardly said to be caused by a sudden exposure to an extremely stressful or traumatic experience. Also prototypical is the explanation that Markowitsch and colleagues invoke for their case. These authors try to explain the amnesia by stating that "[...] even sporadic environmental stress can apparently induce long-lasting brain dysfunction with subsequent massive cognitive deterioration" (Markowitsch et al., 2000, p. 65). Such an explanation might seem intuitively attractive to the layman with little or no knowledge of brain structures, stress and stress reactions, cognitive systems, and known causes of amnesia or other cognitive dysfunctions. However, it is clear that for (neuro)psychologists specialized in memory functioning, this explanation fails to account for amnesia of the sort reported in the case example. What is more, it has been shown that exposure to stress has beneficial 
effects for emotional memory material, making these more memorable in the long run. ${ }^{10}$

Today, there is a broad consensus about the reconstructive nature of episodic memory and its proneness to distortion and confabulation (e.g., Roediger, 1996; Schacter, 1995). However, despite the vigorous research that has been conducted over the years, researchers are still at loss as to what factors contribute to the formation of pseudo-memories, or which type of person is particularly vulnerable to these false recollections. Recently, a study by Payne, Nadel, Allen, Thomas, and Jacobs (2002) has provided preliminary evidence that exposure to acute stress may lead people to remember memory material that, in fact, was never presented to them. These authors had their participants undergo a standard laboratory stress task (i.e., Trier Social Stress Test; see Kirschbaum, Pirke, \& Hellhammer, 1993) or a non-stressful task, followed by the administration of the Deese-RoedigerMcDermott (DRM; Deese, 1959; Roediger \& McDermott, 1995) paradigm. Participants in the stress group exhibited an increase in false recognition of the nonpresented critical lure words. Payne et al. (2002, p. 4) argued that they "[...] suspect that this reflects the role of the hippocampal and PFC systems in contextual remembering, and the modulation of these systems by stress", thereby apparently suggesting that excessive cortisol elevations might account for their results.

The aim of the present dissertation is to investigate the role that acute stress and/or cortisol elevations might play in memory processes in general, and in the development of pseudo-memories in particular. Furthermore, a secondary aim of this dissertation is to take a closer look at what type of pseudo-memory research has been carried out to date, highlight some of the limitations that are inherent in the current research paradigms, and suggest some new directions.

\section{Stress, cortisol, and (pseudo-)memory}

The present dissertation will start with a chapter that examines the effects of acute stress (as induced by a psychosocial challenge test) on declarative memory performance (Chapter 3). Here, we primarily focus on the important role that valence of the memory material plays in these effects and on whether stress may increase levels of falsely recalling extra-list words (i.e., commission errors). In addition, we studied whether post-stress variation in working memory may contribute to the observed memory effects. In the following chapter (Chapter 4), we attempted to replicate findings by Payne and co-workers showing that acute stress not only un-

\footnotetext{
10 A case in point are reports on the quality of memory of concentration camp survivors, showing that these victims of war remember their experiences all too well (e.g., Merckelbach, Dekkers, Wessel, \& Roefs, 2003a, 2003b; Schelach \& Nachson, 2001; Wagenaar \& Groeneweg, 2000; Wessel, Merckelbach, \& Dekkers, 2002).
} 
dermines declarative memory performance, but renders people vulnerable to false recognition of non-presented words as well (Payne et al., 2002). Extending the work of Payne et al., we also measured cortisol responses to determine whether they would affect levels of false recollections.

Subsequently, we investigated whether acute stress and/or the ensuing cortisol responses could alter source monitoring performance regulated by the prefrontal cortex (Chapter 5). To this end, we subjected participants to an internal-internal source monitoring task after they had been exposed to an acute stressor or a nonstressful control task. If acute psychosocial stress and high cortisol stress responses affect source monitoring performance regulated by the prefrontal cortex (PFC) similar to memory processes sustained by the medial temporal lobe, one would expect stressed participants to exhibit more source monitoring errors than nonstressed control participants. On the other hand, given that there are studies showing increased cerebral blood flow in the right PFC during psychological stress (e.g., Wang et al., 2005), one could speculate that acute stress and/or cortisol might yield enhancing effects on source memory performance. Completing the stress, cortisol, and (pseudo-)memory segment of this dissertation, we examined whether the hypothesis that childhood (sexual) abuse leads to lasting neuroendocrine alterations of the hypothalamic-pituitary-adrenal axis possesses empirical credibility (Chapter $6)$. For that reason, we compared the morning cortisol response and diurnal cortisol pattern of women reporting continuous, repressed, or recovered memories of childhood sexual abuse to non-abused control participants.

\section{Opinions and limitations in pseudo-memory research}

In the second segment of this dissertation, the reader is provided with a review of research paradigms intended to elicit so-called "false memories" (Chapter 7). After having closely looked at the methods used by these paradigms, we conclude that many studies have established increased confidence in fictitious childhood events, or have generated false beliefs about them. Yet, they have failed to bring forth concrete, episodic, yet false recollections that are accompanied by images of that event (i.e., pseudo-memories). It is argued that in order for pseudo-memory research to bear resemblance to real world instances of pseudo-memories (e.g., recovered memories), studies are needed that explicitly look at the overt behavioural consequences of the implanted false beliefs and/or memories.

Included in the review of Chapter 7 are studies that make use of the so-called "crashing memory" paradigm. Here, researchers ask individuals whether they have seen a footage of a high-publicity event when, in fact, no such footage exists (e.g., the 1997 car crash that killed Diana, Princess of Wales). By and large, these studies have found that around 50 percent of people are willing to report having seen the non-existent footage. These false reports have sometimes been ascribed as origi- 
nating from pseudo-memories that individuals generate in response to the questions that are posed. Chapter 8 explores the possibility that people reporting to have seen non-existent video fragments actually do not hold pseudo-memories, but that a large proportion of these individuals merely affirm to having seen the aftermath of the event, or that they are simply being misled by the fact that a nonsensical question is being put to them.

Finally, in our General Discussion (Chapter 9), we briefly address how our findings relate to contemporary work and we suggest some directions for future research. 


\section{The effect of acute stress on memory depends on word valence}

This chapter is an adapted version of: Smeets, T., Jelicic, M., \& Merckelbach, H. (2006). The effect of acute stress on memory depends on word valence. International Journal of Psychophysiology, 62, 30-37. 


\section{Summary}

The present study investigated the effect of acute stress on working memory and memory for neutral, emotionally negative, and emotionally positive words in healthy undergraduates. Participants $(N=60)$ were exposed to either the Trier Social Stress Test (stress group) or a non-stressful task (control group). Analyses of salivary cortisol samples taken throughout the study showed elevated glucocorticoid levels after the experimental manipulation in the stress group, but not in the control group. Recall performance was impaired in the stress group, but only so for neutral words. No differences between the stress and control group were found on working memory measures. For the stress group, digit span forward and digit span total scores were associated with correct recall of neutral words. All in all, this study lends further support to the notion that the memory effects of exposure to acute stress depend on the valence of the memory material.

\section{Introduction}

A bulk of animal research has demonstrated that the secretion of glucocorticoids (GCs) from the adrenal cortex during stress may modulate memory storage and consolidation (e.g., de Kloet, Oitzl, \& Joëls, 1999; McGaugh, 2000; Roozendaal, 2000). In animals, GCs can have facilitating (e.g., on aversive conditioning) as well as impairing effects on memory (e.g., de Kloet et al., 1999; Lupien \& McEwen, 1997; McGaugh \& Roozendaal, 2002). Similarly, studies in humans have shown that acute GC administration can have enhancing as well as disruptive effects on memory, depending on several modulatory variables (for reviews, see Het, Ramlow, \& Wolf, 2005; Lupien \& Lepage, 2001; Wolf, 2003). The effects of GCs on memory depend on the differential activation of both mineralocorticoid receptors (MR) and glucocorticoid receptors (GR). It appears that when high affinity MRs are fully occupied while GRs are only partially activated, memory can be facilitated. On the other hand, detrimental effects of high GC levels may occur when GRs become exceedingly saturated during stressful situations (e.g., de Kloet et al., 1999; Oitzl \& de Kloet, 1992; Reul \& de Kloet, 1985). Furthermore, adrenergic activation in the basolateral complex of the amygdala (BLA) and in the hippocampus seems to be required for GCs to impair retrieval (Roozendaal, Hahn, Nathan, de Quervain, \& McGaugh, 2004). Also, activation of noradrenergic mechanisms in the BLA may, in combination with several other brain regions including the hippocampus and prefrontal cortex, mediate the effects of emotional arousal in combination with GC effects on memory functioning (e.g., McGaugh \& Roozendaal, 2002). 
Previous research has shown that men and women not only differ in their endocrinological response to acute psychosocial stress (Kirschbaum, Wüst, \& Hellhammer, 1992; Kudielka \& Kirschbaum, 2005), but also that the effects of acute stress and/or GC elevations can bring about memory effects that differ for men and women (e.g., Takahashi et al., 2004; Wolf et al., 2001b; for review, see Wolf, 2003). In line with animal research (e.g., Roozendaal, 2002), GCs may exert differential effects on various memory phases. In particular, GCs are known to facilitate memory formation (e.g., Buchanan \& Lovallo, 2001) while impairing retrieval (e.g., de Quervain, Roozendaal, Nitsch, McGaugh, \& Hock, 2000; Wolf, Kuhlmann, Buss, Hellhammer, \& Kirschbaum, 2004).

Additionally, prior research has shown that for humans, the effect of stressinduced GC secretion may be detrimental to declarative memory performance, while leaving implicit memory intact. Indeed, there have been studies (e.g., Abercrombie, Kalin, Thurow, Rosenkranz, \& Davidson, 2003; de Quervain et al., 2000; Domes, Rothfischer, Reichwald, \& Hautzinger, 2005; Jelicic, Geraerts, Merckelbach, \& Guerrieri, 2004; Kirschbaum, Wolf, May, Wippich, \& Hellhammer, 1996b; Kuhlmann, Kirschbaum, \& Wolf, 2005a; Lupien et al., 1997; Newcomer, Craft, Hershey, Askins, \& Bardgett, 1994; Newcomer et al., 1999; Tops et al., 2003) illustrating the deleterious effects of acute stress and/or cortisol elevations on declarative memory performance. Kirschbaum and colleagues (1996b), for example, found that impaired declarative memory performance was associated with strong cortisol responses following an acute psychosocial stressor (Experiment 1) and the administration of cortisol (Experiment 2).

Interestingly, some studies have reported that the effect of cortisol on memory performance might depend on the valence of the material being studied. Tops et al. (2003) showed that 2 hours after participants were given either 10mg cortisol or placebo, those who had been given cortisol exhibited impaired recall and recognition of neutral and pleasant words, whereas no difference was found between both groups for unpleasant words. Using a more natural way to elicit high levels of cortisol, Jelicic et al. (2004) exposed half of their participants to a Trier Social Stress Test (TSST; Kirschbaum, Pirke, \& Hellhammer, 1993), which basically consists of a 5 minute free speech followed by a 5 minute mental arithmetic task, while the other half was given a non-stressful control task. Jelicic and colleagues (2004) found that participants in the stress condition exhibited enhanced recall of emotional words, but showed impaired recall of neutral words. In a study by Abercrombie et al. (2003), 90 men were administered either $20 \mathrm{mg}$ or $40 \mathrm{mg}$ of hydrocortisone or placebo and were later asked to rate a list of negative and neutral words for pleasantness and arousal. At a follow-up session 2 days later, Abercrombie et al. found evidence for an inverted U-shaped quadratic trend across stimulus valence, with memory facilitation for both neutral and negative words in the $20 \mathrm{mg}$ hydrocortisone group. These results suggest that only moderate doses of hydrocor- 
tisone enhance memory for words, irrespective of the valence of the studied material.

Recently, Rimmele, Domes, Mathiak, and Hautzinger (2003) had their participants view an emotionally arousing or a neutral story following the administration of $25 \mathrm{mg}$ of hydrocortisone or a placebo. One week later, participants who were given cortisol exhibited enhanced memory for details of the neutral story version, but impaired memory for details of the emotional story. Using a word list containing neutral, pleasant, and unpleasant words, Domes, Heinrichs, Rimmele, Reichwald, and Hautzinger (2004) exposed participants to the TSST either before learning the word list or before retrieval, or were not stressed at all. While free recall was not affected by exposure to the TSST, recognition memory for positive words was impaired for participants who were stressed before retrieval. In a study by Kuhlmann, Piel, \& Wolf (2005b), healthy young males who had learned a word list including neutral, negative, and positive words 24 hours earlier were either assigned to a stress (TSST) or a control condition. In a subsequent free recall task, participants in the stress condition showed impaired memory for the emotionally arousing, but not the neutral words. Most recently, Kuhlmann et al. (2005a) exposed sixteen healthy female participants to either $30 \mathrm{mg}$ hydrocortisone or placebo after they had learned a list containing neutral and negative words. Kuhlmann et al. found that recall for negative words was most strongly impaired at a recall test 5 hours later, while recall for neutral words only tended to be impaired.

In sum, then, results in this research domain on one hand seem to suggest that memory of neutral material is more disrupted by mild psychosocial stress and/or small to moderate cortisol increases (e.g., doses $<20 \mathrm{mg}$ cortisol) than is memory of emotionally provocative material. On the other hand, there have been some divergent findings (e.g., with regard to the effects of cortisol on positive material). One way to account for these divergent findings is to look at the effect of stress-induced cortisol on working memory. Recent studies suggest that impaired memory may - at least in part - be the result of post-stress variation in working memory performance (al' Absi, Hugdahl, \& Lovallo, 2002; Lovallo \& Thomas, 2000; Lupien, Gillin, \& Hauger 1999). That is, high levels of GCs, resulting in occupancy of low-affinity GRs, have been shown to cause a temporary reduction in long-term potentiation (LTP), which in turn may account for post-stress decrements in working memory performance. Moreover, a recent study by Elzinga and Roelofs (2005) found that adrenergic activity is necessary for stress-induced cortisol elevations to impair working memory. The current study thus sought to replicate the finding that stress-induced cortisol increases interfere with memory of neutral material, but not with that of emotional material. We also expected that for the stress group, but not the control group, reduced working memory performance would be associated with impaired medium long-term memory performance. Finally, we also explored the possibility that exposure to acute stress would result in 
elevated levels of spontaneously occurring extra-list intrusions (i.e., commission errors).

\section{Methods}

\section{Participants}

Our sample consisted of 60 healthy undergraduate students (30 men and 30 women). Their mean age was 19.65 years ( \pm 0.24 (SE); range: 17-28). Participants were asked whether they suffered from any cardiovascular diseases or endocrine disorders. If so, they were excluded from the study. All test protocols were approved by the local standing ethics committee of the Psychology Faculty of Maastricht University. All undergraduates participated voluntarily, gave written informed consent, and were paid 10 Euro (approximately 12.5 U.S. dollars) for completing the experiment.

\section{Materials}

Profile of Mood States. Subjective stress was measured using the Anger-Hostility and Tension-Anxiety subscales of the Profile of Mood States-Short Form (POMS; McNair, Lorr, \& Droplleman, 1992). The POMS is a self-report measure that is widely used as a measure of typical and persistent mood reactions to current life situations. Participants indicate to what extent they agree with adjectives describing their current mood or feelings on 5-point scales (anchors: $0=$ not at all, $4=\mathrm{ex}$ tremely). Adjectives include "annoyed", "angry", and "grumpy" for the subscale Anger-Hostility and "nervous", "tensed", and "panicky" for the Tension-Anxiety subscale. The POMS has excellent psychometric properties (see for example, Lezak, 2004c; McNair et al., 1992; Shacham, 1983). The present experiment used a Dutch version of the POMS that has been proven to be both valid and reliable (de Groot, 1991; Wald \& Mellenbergh, 1990).

Working memory testing: Digit Span. The digit span task (see Lezak, 2004b), a subtest of the Wechsler Adult Intelligence Scale-Revised (Wechsler, 1987), requires participants to listen to a series of numbers (ranging from 0 to 9) of increasing length that are read to them at a constant pace of one digit per second. After the last digit has been presented, participants have to repeat the numbers in the exact same (forward condition) or the exact reverse (backward condition) order. On each successful attempt, the number of digits per list increases. Thus, the digit span involves auditory attention and depends on working retention capacity. Whenever a participant fails to reproduce a certain list of numbers, a second attempt with another list of digits of equal length follows. When a participant fails to accurately reproduce a list of numbers on two successive trials (e.g., failing to reproduce a 6item list on two successive trials), the task ends. On average, people recall about 7 
items (Miller, 1956). Raw scores for the digit span forward and backward reflect the maximum number of digits correctly recalled. Digit span forward and backward scores are summed to obtain a digit span total score.

Declarative memory testing: 30 Word Verbal Learning Task. The 30-word Verbal Learning Task (30WVLT) is an adapted version of the Rey Auditory Verbal Learning Task (originally published by Rey, 1964; see also Deelman, Brouwer, van Zomeren, \& Saan, 1980; Lezak, 2004a) designed to assess learning capabilities, maximal and total capacity, and storage as well as retrieval efficiency of verbal material. Participants are required to listen to and remember as many words as possible from a list of 30 words, read out to them at a pace of one word every 2 seconds. As the valence of stimuli may differentially affect recall (e.g., Tops et al., 2003), the list of presented stimuli words comprised 10 positive emotional (e.g., "peace"), 10 negative emotional (e.g., "cancer"), and 10 neutral (e.g., "stone") words, all acquired early in life. All words were chosen from Hermans and De Houwer's (1994) list of words for which they had 352 first year students rate the subjective familiarity and affectivity of 740 Dutch words. Based on these data, all three word categories (positive emotional, negative emotional, and neutral) differed significantly for affectivity $[F(2,27)=1491.15 ; p<.001]$, but not for familiarity or word length $([F(2,27)=1.91 ; p=.17]$ and $[F(2,27)<1 ; p=.71]$, respectively). Presentation order of the 30 words was randomized. ${ }^{11}$ After the list had been read to participants, they were instructed to freely reproduce as many words as possible. However, they were not told that the list consisted of 30 words. Next, the 30 words were read out again and participants were required to reproduce them. After 3 such 'learning' trials had taken place, a retention interval of 30 minutes was maintained after which participants were given a delayed recall and recognition task. Recall performance was scored as the proportion correctly recalled positive, negative, and neutral words, with higher scores indicating superior correct recall performance. The recognition task consisted of 60 words (30 presented and 30 non-presented). The 30 new, non-presented words were also taken from Hermans and De Houwer (1994) and included 10 emotionally positive, 10 emotionally negative, and 10 neutral words. A one-way Analysis of Variance (ANOVA) showed that the 10 new positive emotional words did not differ significantly from the 10 old words that were presented in the word learning task with regard to affect, familiarity, or word length (all F's < 1). Likewise, the old and new emotionally negative words did not differ in terms of affect, familiarity, or word length (all $F$ 's $<2.5$; all $p$ 's $>.10$ ). Neither were there differences between new and old neutral words (all $F$ 's $<1$ ). Order of old and new words in the recognition task was random. Recognition performance was assessed by calculating a Discrimination Index $\operatorname{Pr}$ (Snoddgrass \& Corwin, 1988). Pr indicates the ability to discriminate old from new words and was com-

\footnotetext{
11 The list of stimuli words used in this experiment can be obtained from the author.
} 
puted as follows: $\operatorname{Pr}=$ (hit rate - false alarm rate). Higher $\operatorname{Pr}^{2}$ s are indicative of enhanced recognition performance.

\section{Saliva sampling and free cortisol analysis}

Cotton Salivettes (Sarstedt ${ }^{\circledR}$, Nümbrecht, Germany) were used to obtain the cortisol samples. The uncentrifuged saliva samples were stored at $-40^{\circ}$ Celsius immediately upon collection. Salivary free cortisol levels were determined in duplicate by direct radioimmunoassay (RIA; University of Liège), including a competition reaction between ${ }^{125}$ iodohistamine-cortisol and anti-cortisol serum made against the 3CMO-BSA conjugate. Via a conventional 'second antibody' method, separation of free and antibody-bound ${ }^{125 i o d o h i s t a m i n e-c o r t i s o l ~ w a s ~ p e r f o r m e d ~ a f t e r ~ o v e r n i g h t ~}$ incubation at $4^{\circ}$ Celsius of $100 \mu \mathrm{l}$ of saliva. In order to reduce sources of variability, all 4 samples from an individual were analyzed in the same assay. Mean intraand inter-assay coefficients of variation were less than 4.3 percent and 8.3 percent, respectively.

\section{Design}

A 2-group between-subject design was employed. Half of the participants (i.e., 15 men and 15 women) were exposed to the TSST (Kirschbaum et al., 1993), while the other half were assigned to a non-stressful control group. The two groups did not differ with respect to age (stress group $19.47 \pm 0.24$ years (mean $\pm S E$ ); control group $19.83 \pm 0.40$ years; $[t(58)<1 ; p=.44])$.

\section{Procedure}

All sessions were run between $14.00 \mathrm{~h}$ and $17.00 \mathrm{~h}$ to obtain comparable and stable basal cortisol levels. All participants were tested individually. To allow for objective controlled cortisol sampling, participants refrained from food, drinks, smoking, and heavy exercise at least 1 hour prior to the test phase. Upon arrival in the laboratory, participants were informed about the nature and procedure of the experiment and signed a consent form. After a short rest period, a first cortisol sample (baseline; $\mathrm{t}-5$ ) was obtained using Salivettes and the POMS was administered a first time. In order to eliminate anticipatory stress reactions (e.g., Kirschbaum et al., 1992) which could affect baseline cortisol measurement, participants were told about the task they had to perform subsequently only after the baseline cortisol measurement had already been performed. Male and female participants were pseudo randomly assigned to either the stress or the control group. In the stress group, participants were exposed to the TSST (Kirschbaum et al., 1993). The TSST protocol basically consists of a 10 minute preparation period, a 5 minute free speech, and a 5 minute mental arithmetic task in front of an audience while being videotaped. The TSST has repeatedly been shown to be a valid and reliable procedure to induce physiological stress responses in children, young as well as elderly 
adults (e.g., Kirschbaum et al., 1992; Kudielka, Buske-Kirschbaum, Hellhammer, \& Kirschbaum, 2004a; Kudielka, Schommer, Hellhammer, \& Kirschbaum, 2004b). Moreover, in a recent meta-analysis, the TSST was found to provoke the most robust physiological stress responses (i.e., cortisol stress responses) as compared to several other laboratory stress tasks (Dickerson \& Kemeny, 2004). Participants in the control group were given a non-stressful filler task that consisted of filling out some questionnaires and playing a computer card game. The TSST and filler task had a similar duration.

Subsequent to the TSST or filler task, a second cortisol sample $(t+15)$ was obtained and the POMS was again administered. Afterwards, the 30 WVLT and digit span task were administered. During the 30 minute retention interval for the delayed recall task of the 30WVLT, non-verbal filler tasks were given. A third $(\mathrm{t}+35)$ and fourth $(\mathrm{t}+55)$ cortisol sample were obtained 20 minutes after finishing the TSST and at the end of the test session, respectively. The total time of the experiment did not exceed 1 hour. Finally, participants were debriefed, paid, and thanked for their participation.

\section{Statistical analyses}

Cortisol responses were analyzed using a 2 (group) $\times 2(\operatorname{sex}) \times 4$ (time: $\mathrm{t}-5$ vs. $\mathrm{t}+15$ vs. $\mathrm{t}+35$ vs. $\mathrm{t}+55)$ Analysis of Variance (ANOVA) with time as repeated factor. A 2 (group) $\times 2$ (sex) $\times 2$ (time: pre-test vs. post-test) ANOVA was used to check feelings of distress (POMS) following the TSST or filler task. Digit span performance was analyzed using 2 (group) $\times 2$ (sex) ANOVA. Learning, recall, and recognition performance for the negative, neutral, and positive words were analyzed using 2 (group) $\times 2$ (sex) $\times 3$ (valence: positive vs. negative vs. neutral) ANOVA's, with valence as repeated factor. Additionally, delta increases in cortisol (i.e., cortisol responses) defined as peak cortisol level $(\mathrm{t}+15, \mathrm{t}+35$ or $\mathrm{t}+55)$ minus baseline cortisol level were computed for each participant individually and correlated (Pearson correlations, two-tailed) with memory performance measures within the stress group. Finally, to investigate the role of working memory in acute stress effects on declarative memory performance, recall and recognition performance on the word learning task was correlated with digit span scores for the stress and control group separately (two-tailed). Alpha was set at .05 unless specified otherwise. When sphericity assumptions were violated, Greenhouse-Geisser corrected $p$-values are reported. 


\section{Results}

\section{Baseline cortisol analyses}

Cortisol data from one female participant in the control condition was lost due to saliva sampling problems. An independent samples $t$-test showed no differences in baseline cortisol levels between the stress group and the control group, means being $4.40 \mathrm{nmol} / 1( \pm 0.36(\mathrm{SE}))$ and $4.26 \mathrm{nmol} / 1( \pm 0.54)$, respectively $[t(57)<1 ; p=$ .83].

\section{Glucocorticoid stress responses}

Repeated measures ANOVA revealed that salivary free cortisol levels were significantly higher in the experimental (i.e., stress) than in the control group, as indicated by main effects of time $[F(3,165)=6.69 ; p=.002]$, group $[F(1,55)=6.04 ; p=$ $.017]$, and the critical Group $\times$ Time interaction $[F(3,165)=4.81 ; p=.011]$. No other main or interactive effects were found. Bonferroni corrected post-hoc tests indicated that levels of cortisol were significantly higher in the stress group at $\mathrm{t}+15$ $[t(57)=2.82 ; p=.007], \mathrm{t}+35[t(57)=2.53 ; p=.015]$, as well as at $\mathrm{t}+55[t(57)=$ $2.60 ; p=.013$ ]. Figure 3.1 shows mean salivary cortisol levels throughout the experiment for both groups.

\section{Psychological assessment of subjective stress}

Mean scores on the POMS subscales Anger-Hostility and Tension-Anxiety are shown in Table 3.1. As to the Anger-Hostility subscale scores, significant main effects of group $[F(1,56)=6.18 ; p=.016]$, time $[F(1,56)=4.15 ; p=.046]$, and the crucial Group $\times$ Time interaction $[F(1,56)=4.84 ; p=.039]$ were found, in the absence of other main or interactive effects. Similarly, significant effects of group $[F(1,56)=12.64 ; p=.001]$, time $[F(1,56)=10.78 ; p=.002]$, and the Group $\times$ Time interaction $[F(1,56)=31.00 ; p<.001]$ were observed for Tension-Anxiety subscale scores. These differences indicate that experimental participants experienced significantly more stress than control participants.

Table 3.1. Mean scores on subscales Anger-Hostility (AH) and Tension-Anxiety (TA) of the Profile of Mood States before (Pre-AH and Pre-TA) and after (Post-AH and Post-TA) the experimental manipulation. Standard errors of mean are given between parentheses.

\begin{tabular}{|l|c|c|c|c|}
\hline & Pre-AH & Post-AH & Pre-TA & Post-TA \\
\hline Control group & $0.90(0.30)$ & $0.87(0.35)$ & $5.53(0.39)$ & $4.73(0.42)$ \\
\hline Stress group & $1.63(0.70)$ & $3.37(0.72)$ & $5.43(0.49)$ & $8.53(0.45)$ \\
\hline
\end{tabular}




\section{Stress-induced cortisol responses}

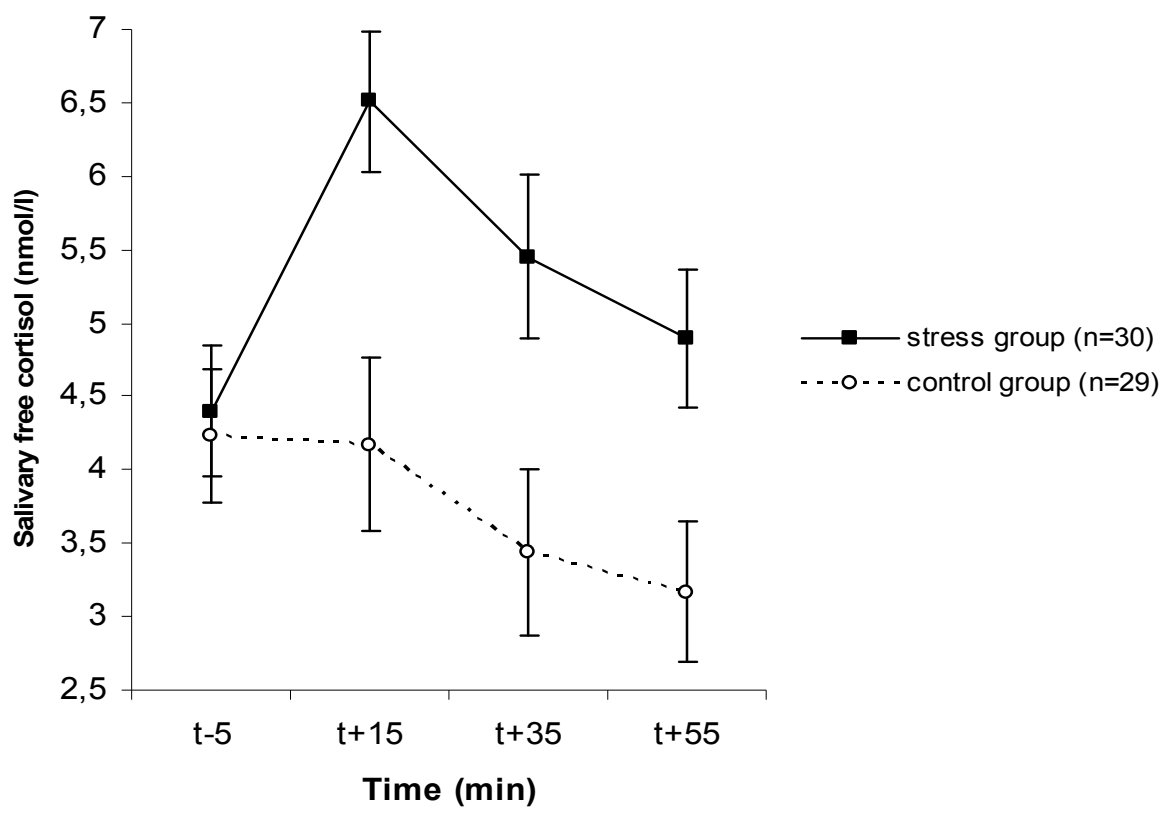

Figure 3.1. Mean free salivary cortisol response (nmol/l) before stress manipulation ( $\mathrm{t}-5)$, directly after stress exposure (TSST; $t+15), 20$ minutes after stress exposure $(t+35)$, and at the end of testing $(\mathrm{t}+55)$.

\section{Memory performance}

Digit Span. Descriptive results on digit span measures can be found in Table 3.2. For digit span forward, ANOVA revealed a main effect of sex $[F(1,56)=6.45 ; p=$ $.014]$, but not of group $[F(1,56)<1 ; p=.99]$, with men having slightly higher digit span forward scores than did women. The Group $\times$ Sex interaction just fell short of significance $[F(1,56)=3.63 ; p=.06]$. No main effect of group or sex, or a Group $\times$ Sex interaction was found for digit span backward (all $F$ 's $<1$ ).

Word Learning Task - Recall performance. Learning performance as measured by overall proportion of recalled words after each learning trial did not differ between groups at learning trial 1 (means being .40 and .41 for the stress and control group, respectively; $[F(1,56)<1 ; p=.66]$ ), learning trial 2 (means being .59 and .60 for the stress and control group, respectively; $[F(1,56)<1 ; p=.79])$, or learning trial 3 (means being .72 and .74 for the stress and control group, respectively; $[F(1,56)<$ $1 ; p=.51])$. No other main or interaction effects were found in learning performance (all $F$ s $<1$ ). 
Mean percentage correctly recalled words for the delayed recall task can be found in Table 3.2. ANOVA yielded a significant main effect of valence $[F(2,56)=$ 8.23; $p<.001]$ and a significant Group $\times$ Valence interaction $[F(2,56)=3.74 ; p=$ .03]. No other main or interactive effects were detected. Bonferroni corrected post-hoc analyses showed that recall of neutral words was significantly impaired in the stress group $(p<.01)$, while no such impairment was found for negative or positive words (both $p$ 's $>.05$ ). Furthermore, we observed that the stress group made more list intrusions or commission errors (i.e., non-presented words being recalled) than the control group, $[F(1,56)=4.52 ; p<.05]$.

Table 3.2. Mean scores on Digit Span Forward, Backward, and Total, and mean recall and recognition performance $(P r)$ for neutral, positive, and negative words for the stress and control group. Standard errors of mean are given between parentheses.

\begin{tabular}{|l|c|c|}
\hline \multicolumn{1}{|l|}{} & Control group & Stress group \\
\hline Digit Span & & \\
\hline Forward condition & $6.83(.28)$ & $6.83(.19)$ \\
\hline Backward condition & $5.70(.28)$ & $5.63(.23)$ \\
\hline Total score & $12.53(.51)$ & $12.47(.33)$ \\
\hline Mean percentage correct recall & & \\
\hline Neutral words & \\
\hline Positive words & $.72(.02)$ & $.60(.03)$ \\
\hline Negative words & $.63(.03)$ & $.63(.03)$ \\
\hline Mean number of commissions ${ }^{\mathrm{b}}$ & $.74(.03)$ & $.69(.04)$ \\
\hline Mean recognition performance $\mathbf{( P \boldsymbol { r } )}$ & $.27(.15)$ & $.70(.14)$ \\
\hline Neutral words ${ }^{\mathrm{a}}$ & & \\
\hline Positive words & $.99(.01)$ & $.91(.02)$ \\
\hline Negative words & $.94(.02)$ & $.91(.03)$ \\
\hline
\end{tabular}

Notes: ${ }^{\mathrm{a}} p<.01$, two-tailed; ${ }^{\mathrm{b}} p<.05$, two-tailed

Word Learning Task - Recognition performance. As to recognition performance (Pr), ANOVA showed significant main effects of group $[F(1,56)=4.15 ; p<.05]$ and valence $[F(2,56)=6.70 ; p=.002]$ in the absence of other main or interactive effects (all $p$ 's $>.10)$. Bonferroni corrected post-hoc analyses showed that the control group had higher discrimination indices than the stress group.

Correlations between digit span and recall and recognition performance on the Word Learning Task. Correlations between digit span forward, backward, and total scores and recall and recognition performance for positive, negative, and neutral words were computed. For the stress group, this yielded significant correlations between correct recall of neutral words and digit span forward and total scores $(r=.41 ; p<.05$ 
and $r=.36 ; p<.05$, respectively). For the control group, no meaningful correlations emerged (all $r^{2}$ s $<.30$; all $p$ 's $>.10$ ).

Within stress group correlations between memory performance measures and individual delta cortisol responses. ${ }^{12}$ Delta cortisol responses were negatively related to digit span backward scores $(r=-.39 ; p=.03)$. All other correlations remained non-significant (all p’s $>.05)$.

\section{Discussion}

The present study was set out to further evaluate the effects of acute stress on declarative memory performance reported in previous studies (e.g., Kirschbaum et al., 1996b) and the precise role of word valence in these effects (e.g., Tops et al., 2003). The main results of our study can be summarized as follows. Participants in the stress group did not differ from controls with respect to digit span measures. They did, however, show impaired recall of neutral words in the absence of learning differences between both groups. No differences between the groups were found for recall of positive emotional or negative emotional words. Additionally, participants in the stress group made more commission errors than did control participants. The recognition test showed that participants in the stress groups performed worse than those in the control group. Moreover, in the stress but not the control group, post-stress performance on forward and total digit span was significantly correlated with correct recall of neutral words.

The finding that memory for neutral words was impaired for stressed participants accords well with previous findings obtained by Kirschbaum et al. (1996b), Lupien et al. (1997), Wolf et al. (2001a), Jelicic et al. (2004), and Tops et al. (2003). In contrast to the work by Buchanan and Lovallo (2001) and Jelicic et al. (2004), however, cortisol alterations in this study did not lead to enhanced memory for positive or negative emotional words. Note, however, that the cortisol levels reported by Buchanan and Lovallo were much higher than those reported here, due to the exogenous administration of high doses of cortisol. Also, these results are at odds with findings suggesting impaired memory for emotional material (e.g., Domes et al., 2004; Kuhlmann et al., 2005a, 2005b; Rimmele et al., 2003). It should be acknowledged here that for recognition performance, the crucial interaction between group and valence remained non-significant despite significant effects of group and valence. This suggests that recall and recognition tasks may yield qualita-

\footnotetext{
12 To further evaluate whether cortisol levels had a modulating effect on the present data, we conducted 3(group: controls vs. low responders vs. high responders) $\times 2$ (sex) ANOVA's on our dependent (i.e., memory performance) measures. Results were similar to those reported in previous sections, suggesting that cortisol levels do not modulate the current study's results.
} 
tively different interaction effects between exposure to acute stress and the valence of the memory material.

In addition to the memory impairing effect of acute stress on memory for neutral words, we found that participants in the stress group committed significantly more commission errors during free recall. This is in accordance with findings from Wolkowitz et al. (1990), who reported that participants exposed to $80 \mathrm{mg} /$ day of prednisone for 5 consecutive days made more commissions in a verbal memory task than participants who had received a placebo.

The present study failed to find differences between the stress and control group on digit span performance. Our data thus replicate the findings of Hoffman and al' Absi (2004) and Kuhlmann et al. (2005b), who reported no effects of acute stress on digit span performance. It should be noted here that Elzinga and Roelofs (2005) recently found that adrenergic activity is essential for cortisol elevations to result in working memory deficits. These results might explain why in the present study no impairments in working memory performance were noted despite the fact that cortisol levels were significantly elevated. However that may be, the current data do confirm our hypothesis that working memory performance following acute stress is associated with medium long-term memory performance. This association was evident for the stress group, while no such association was found for the controls. However, the fact that stress and control group did not differ in digit span or learning performance makes it impossible to confidently assert that post-stress variation in working memory performance adds to the observed memory effect.

Some notes on the limitations of this study are in order. First of all, although the stressed group showed significant increases in cortisol, it might be argued that levels of cortisol in the stressed group were insufficiently high to bear relevance to high levels of cortisol under circumstances of real-life stress. Secondly, it can be argued that the current study relied only on a limited number of memory measures, thereby failing to raise the matter of medium long-term memory versus long-term memory effects following acute psychosocial stress. Therefore, the effect of acute cortisol elevations on memory over longer delays (e.g., several weeks) awaits to be determined. Also, it should be acknowledged that for the female participants, cortisol responses may have been influenced by menstrual cycle and the use of oral contraceptives (Kirschbaum et al., 1992). It is also worthy of note that previous research has shown that GCs may differentially affect the various memory phases. That is, while GCs have been shown to enhance memory formation (e.g., Buchanan \& Lovallo, 2001), impairments in retrieval have also been reported (e.g., de Quervain et al., 2000; Wolf et al., 2004). Roozendaal (2002) suggests that the BLA under stressful circumstances turns the brain into a memory-consolidation state, while concurrently destabilizing future retrieval attempts. This could be important given the fact that the present study involved only one session. Indeed, strictly from a theoretical viewpoint, memory-facilitating effects of moderate GC increases on memory formation might even suppress or eliminate the detrimental effects of 
such GC's on retrieval processes. However, in the present study this would have been rather unlikely given the fact that no differences in learning performance were observed. Another factor that should be taken into account is that cortisol appears to lead to impairing effects when studies are conducted in the morning, while no or slightly enhancing effects are found in the afternoon (e.g., Het et al., 2005; Lupien, Wilkinson, Briere, Menard, Ng Ying Kin, \& Nair, 2002b). Therefore, it awaits to be seen whether the present results can be generalized to other times of day. Future research should take these limitations into account.

In sum, then, the present data lend further support to the idea that valence of the memory material modulates the effects of acute stress on declarative memory performance. Our results also suggest that even acute exposure to heightened levels of cortisol may under some circumstances result in pertinent impairments in memory for neutral material, but that emotional material seems relatively immune to the memory undermining effects of heightened cortisol. Future studies should further delineate the precise boundaries of this effect and explore whether certain modulating factors (e.g., retention delay, age) may account for the inconsistent findings in this research domain. Research into acute stress effects may help us to advance our understanding of the complex role GCs and stimulus valence play in the regulation of human memory functioning. 


\section{4}

\section{Stress-induced cortisol responses, sex differences, and false recollections in a DRM paradigm}

This chapter is an adapted version of: Smeets, T., Jelicic, M., \& Merckelbach, H. (2006). Stress-induced cortisol responses, sex differences, and false recollections in a DRM paradigm. Biological Psychology, 72, 164-172. 


\section{Summary}

The current studies investigated whether acute stress potentiates false recollections in a Deese-Roediger-McDermott (DRM) paradigm, and whether sex differences modulate these effects. Participants were assigned to either a stress (Trier Social Stress Test) or a control group. Subsequently, they were subjected to DRM word lists and probed for recall and recognition. Results showed no differences between the stress and control group on measures of false recollections (study $1 ; N=60$ ). Even though correct recall was impaired by acute stress, there were no differences between high or low cortisol responders and controls on false recall or recognition rates (study 2; $N=92$ ). These results suggest that cortisol responses do not directly potentiate false recollections. Neither in study 1 nor in study 2 did we find any evidence that the effects of cortisol on false recollections are different in men and women, although there was an indication that independent of stress men produced more commission errors.

\section{Introduction}

Using a large number of different settings and paradigms, so-called pseudo-memories have been shown to be fairly easy to elicit and bear clinical as well as legal implications. One paradigm intended to elicit false recall and recognition is the DeeseRoediger-McDermott paradigm (DRM; Deese, 1959; Roediger \& McDermott, 1995). Here, participants are presented with lists of semantic associates (e.g., bed, tired, dream, snooze, doze, nap). After the list has been presented, participants are given a free recall task. Subsequently, a test probing for recognition of presented words (e.g., dream), non-presented unrelated distracter words (e.g., rain), and a semantically related non-presented word termed the "critical lure" (in this case the word sleep) follows. Typically, people often falsely recall and/or recognize semantically related but non-presented words (i.e., the critical lures) as having been presented to them in the list learning task (e.g., McDermott, 1996; Roediger, Jacoby, \& McDermott, 1996; Roediger \& McDermott, 1995; Roediger, Watson, McDermott, \& Gallo, 2001). Thus, the DRM paradigm has the capacity to reliably elicit striking memory illusions (see, for further examples, Blair, Lenton, \& Hastie, 2002; Roediger \& McDermott, 2000).

Recent findings from Payne and co-workers (Payne, Nadel, Allen, Thomas, \& Jacobs, 2002) suggest that stress may increase false recognition rates in the DRM paradigm. After they were exposed to the Trier Social Stress Test (TSST; Kirschbaum, Pirke, \& Hellhammer, 1993) or a filler task, participants had to listen to 20 DRM word lists, each followed by a computerized recognition task. Compared to controls, participants exposed to the TSST showed elevated rates of false recog- 
nition for the critical lures. Payne et al. (2002, p. 5) concluded that "When stress disrupts hippocampal and/or prefrontal cortex processing, false recognition increases dramatically. [...] Whatever the precise localisation of stress effects on false recognition, this study has demonstrated that moderate psychological stress renders subjects unable to distinguish between "true" and "false" memories in the DRM paradigm." This conclusion implies that people under stressful circumstances are more vulnerable to specific memory distortions, due to secretion of glucocorticoid (GC) stress hormones.

In line with animal research (e.g., de Kloet, Oitzl, \& Joëls, 1999; McGaugh \& Roozendaal, 2002), studies in humans have shown that acute GC administration can have enhancing as well as disruptive effects on memory, depending on several modulatory variables (for reviews, see Het, Ramlow, \& Wolf, 2005; Lupien \& Lepage, 2001; Wolf, 2003). Furthermore, GCs are known to facilitate memory formation (e.g., Buchanan \& Lovallo, 2001) while impairing retrieval (e.g., Wolf, Kuhlmann, Buss, Hellhammer, \& Kirschbaum, 2004). Also worth mentioning here is that recent work suggests that emotional valence might influence the memory effects GCs can have (e.g., Kuhlmann, Kirschbaum, \& Wolf, 2005a; Tops et al., 2003; Wolf et al., 2004).

Animal (e.g., Conrad et al., 2004; Luine, 2002; Wood, Beylin, \& Shors, 2001; Wood \& Shors, 1998) as well as human studies have found evidence for sex differences in the effects of stress hormones (e.g., cortisol) on memory functioning. For example, Seeman and colleagues (Seeman, McEwen, Singer, Albert, \& Rowe, 1997) found that higher basal levels of cortisol were associated with impaired memory performance among elderly women, but not among men. In line with these results, Wolf and colleagues (Wolf, Kudielka, Hellhammer, Hellhammer, \& Kirschbaum, 1998) noted that exposure to the TSST following Dehydroepiandrosterone (DHEA) treatment significantly impaired memory performance in elderly women, but not in elderly men. In a follow-up study, Wolf and co-workers (Wolf, Schommer, Hellhammer, McEwen, \& Kirschbaum, 2001b) found that within a group of young adults exposed to the TSST, cortisol increases were negatively correlated with memory performance. Further analysis, however, revealed that this effect was carried by a strong negative correlation in men while no such correlation was found among women.

Taken together, human studies suggest that stress-induced cortisol increases may have a corrupting effect on memory performance and that sex may modulate the effects of stress on memory, but that the precise form that this modulation may take is far from clear. Looking at potential sex differences in memory performance after stress is important given the mixed results in this domain of research. With this in mind, the current studies were carried out. More specifically, using equal numbers of men and women distributed over stress and control groups, we sought to replicate the results of Payne et al. (2002). Furthermore, we examined whether the modulating effect of sex on memory functioning also trans- 
lates into commission errors during free recall (i.e., falsely recalling non-presented words not related to the study words). Additionally, we obtained objective cortisol sampling data to investigate whether there is an interaction between sex, stressinduced cortisol responses, and false recall and recognition of the critical lures following a DRM paradigm.

\section{STUDY 1}

\section{Methods}

\section{Participants}

Our sample consisted of 60 young healthy undergraduate students (30 men and 30 women). Their mean age was 19.91 years $(S D=3.32)$. Participants were asked whether they suffered from any cardiovascular diseases, endocrine disorders, or asthma. If so, they were excluded from the study. In study 1 no attempt was made to control for menstrual cycle or the use of oral contraceptives. All test protocols were approved by the standing ethics committee of the Psychology Faculty of Maastricht University. All participants signed a written informed consent and were given a small financial compensation (10 euro; approximately 12.5 U.S. dollars) for completing the experiment.

\section{Materials}

Profile of Mood States. Subjective stress was measured using the Anger-Hostility and Tension-Anxiety subscales of the Profile of Mood States-Short Form (POMS; McNair, Lorr, \& Droppleman, 1992). The POMS is a self-report measure that is widely used as a measure of typical and persistent mood reactions to current life situations. Participants indicate to what extent they agree with adjectives describing their current mood or feelings on 5-point scales (anchors: $0=$ not at all, $4=e x$ tremely). Adjectives include "annoyed", "angry", and "grumpy" for the subscale Anger-Hostility and "nervous", "tensed", and "panicky" for the Tension-Anxiety subscale. The POMS has excellent psychometric properties (see for example, Lezak, 1995; McNair et al., 1992; Shacham, 1983). The present experiment used a Dutch version of the POMS that has been proven to be valid and reliable (de Groot, 1991; Wald \& Mellenbergh, 1990). For practical consideration and following Van Honk, Kessels, Putman, Jager, Koppeschaar, \& Postma (2003), we did not use the total POMS.

Deese-Roediger-McDermott paradigm (DRM). We used a version of the DRM paradigm (Roediger \& McDermott, 1995) in which participants were read out 12 semantically related wordlists. After each list they were given a free recall test. After all lists 
had been presented, participants were probed for recognition memory using a 72 item recognition task consisting of 36 studied words and 36 non-studied words. Non-studied words included 12 critical lures and 24 unrelated distracter words. Participants had to indicate whether the words were presented to them ("old") or not ("new"). Five dependent measures were derived from the DRM paradigm: (1) the number of commission errors (i.e., confabulated extra list words not related to the study words) on the free recall task, (2) proportion correct recall of presented words, (3) proportion falsely recalled critical lure words, (4) proportion correct recognition (i.e., recognizing presented words as "old" and recognizing distracter words as "new"), and (5) proportion falsely recognized critical lures (i.e., nonpresented critical lures judged as "old").

Trier Social Stress Test (TSST). The TSST (Kirschbaum et al., 1993) is a psychosocial challenge test which basically consists of a 10 minute preparation period, a 5 minute free speech, and a 5 minute mental arithmetic task in front of an audience while being videotaped. The TSST is a valid and reliable procedure to induce physiological stress responses in children, young as well as elderly adults (e.g., Kirschbaum, Wüst, \& Hellhammer, 1992; Kudielka, Buske-Kirschbaum, Hellhammer, \& Kirschbaum, 2004a; Kudielka, Schommer, Hellhammer, \& Kirschbaum, 2004b). In a recent meta-analysis, the TSST was found to provoke the most robust physiological stress responses (i.e., cortisol stress responses) relative to various other laboratory stress tasks (Dickerson \& Kemeny, 2004). The current study closely followed the TSST protocol as described by Kirschbaum et al. (1993).

\section{Saliva sampling and biochemical analyses}

Cortisol data were obtained using cotton Salivettes (Sarstedt ${ }^{\circledR}$, Nümbrecht, Germany). The uncentrifuged saliva samples were stored at $-40^{\circ}$ Celsius immediately upon collection. Salivary free cortisol levels were determined in duplicate by direct radioimmunoassay (RIA; University of Liège, Belgium), including a competition reaction between ${ }^{125}$ iodohistamine-cortisol and anti-cortisol serum made against the 3-CMO-BSA conjugate. After overnight incubation at $4^{\circ}$ Celsius of $100 \mu \mathrm{l}$ of saliva, separation of free and antibody-bound ${ }^{125}$ iodohistamine-cortisol was performed via a conventional 'second antibody' method. In order to reduce sources of variability, all 4 samples taken from each participant (see below) were analyzed in the same assay. Mean intra- and inter-assay coefficients of variation were less than 4.3 percent and 8.3 percent, respectively.

\section{Design}

Participants were quasi-randomly assigned to one of two groups. Half of the participants (i.e., 15 men and 15 women) were exposed to the TSST (Kirschbaum et al., 1993) serving as the stress group, while the other half were assigned to a control group that included a filler task. The two groups did not differ with respect to age (stress group $M=19.97$ years, $S D=4.20$; control group $M=19.86$ years, $S D=$ 
2.18). Thus, the experiment was designed according to a 2 (group: stress vs. control) $\times 2$ (sex: men vs. women) set-up.

\section{Procedure}

Experimental sessions were run between $14.00 \mathrm{~h}$ and $17.00 \mathrm{~h}$ when basal cortisol levels are low and stable. Participants were tested individually. To allow for objective controlled cortisol sampling all participants were deprived of food, drinks, smoking, and heavy exercise at least 1 hour prior to the test phase. After arrival in the laboratory, they were informed about the memory tests and subsequently signed a consent form. A first cortisol sample (pre-stress) was then obtained using a Salivette (Sarstedt ${ }^{\circledR}$, Nümbrecht, Germany) and the POMS was administered a first time. In order to eliminate anticipatory stress reactions (e.g., Kirschbaum et al., 1992) which could affect pre-stress cortisol measurement, participants were told about the stress or filler task they would have to perform subsequently after the pre-stress cortisol measurement had been obtained. Participants in the stress group were exposed to the TSST, while the control group was given a filler task that consisted of filling out some questionnaires and playing a computer card game or minesweeper. TSST and filler task had a similar duration.

Subsequent to the TSST or filler task, a second cortisol sample $(t+20)$ was obtained and the POMS was again administered. Afterwards, participants were exposed to a DRM task consisting of 12 DRM lists and free recall tests followed by a recognition task. A third cortisol sample $(t+35)$ was taken after the 6th DRM list (i.e., 15 minutes after the TSST) and a fourth and final sample $(t+50)$ was obtained at the end of the test session. All memory measures were completed after about 40 minutes and total time of the entire session did not exceed 1 hour. Participants were debriefed, paid, and thanked for their participation.

\section{Statistical analyses}

A 2 (group) $\times 2$ (sex) $\times 2$ (time: pre- vs. post-manipulation) Analysis of Variance (ANOVA) with time as repeated factor was used to evaluate feelings of subjective stress (POMS). Cortisol responses were analyzed using a 2 (group) $\times 2$ (sex $) \times$ 4(time: pre-stress vs. $\mathrm{t}+20$ vs. $\mathrm{t}+35$ vs. $\mathrm{t}+50$ ) ANOVA, with cortisol sample as repeated factor. Additionally, delta increases in cortisol (i.e., cortisol responses) defined as peak cortisol level $(t+20, t+35$, or $t+50)$ after the TSST or filler task minus pre-stress cortisol level were computed for each participant individually. Delta responses were analyzed using a 2 (group) $\times 2$ (sex) ANOVA. A responder rate of participants showing a cortisol increase larger than $2.5 \mathrm{nmol} / 1$ (see, for example, Kirschbaum et al., 1993; Kirschbaum, Wolf, May, Wippich, \& Hellhammer, 1996b; Schommer, Hellhammer, \& Kirschbaum, 2003), which is thought to reflect a cortisol secretory episode (Van Cauter \& Refetoff, 1985), was calculated. For each dependent measure a 2 (group) $\times 2$ (sex) ANOVA was employed. Finally, for participants exposed to the TSST Spearman rho correlations were computed be- 
tween false recall and recognition rates and delta cortisol increases. When sphericity assumptions were violated, Greenhouse-Geisser corrected $p$-values are reported. Alpha was set at .05 unless specified otherwise, and adjusted (Bonferroni) for multiple comparisons where necessary.

\section{Results}

\section{Manipulation check}

Table 4.1 shows sum scores on the Anger-Hostility and Tension-Anxiety POMS subscales indicating feelings of subjective stress. As expected, a significant Group $\times$ Time interaction was found $[F(1,54)=12.25 ; p=.001]$ for the Anger-Hostility subscale. No other main or interaction effects were found. For the TensionAnxiety subscale, ANOVA yielded significant effects of time $[F(1,54)=23.87 ; p<$ $.001]$, group $[F(1,54)=12.31 ; p=.001]$, and the anticipated Group $\times$ Time interaction $[F(1,54)=70.42 ; p<.001]$. All other main or interactive effects remained nonsignificant. Both ANOVA's indicate that men as well as women in the stress group experienced more stress than did control participants after the TSST or filler task.

Table 4.1. Study 1 sum scores on subscales Anger-Hostility and Tension-Anxiety of the Profile of Mood States before (Initial) and after (Post-test) experimental manipulation or control filler task for men and women separately. Standard deviations are given in parentheses.

\begin{tabular}{|l|c|c|c|c|}
\hline & \multicolumn{2}{|c|}{ MEN } & \multicolumn{2}{c|}{ WOMEN } \\
\hline & $\begin{array}{c}\text { Control } \\
(n=14)\end{array}$ & $\begin{array}{c}\text { Stress } \\
(n=15)\end{array}$ & $\begin{array}{c}\text { Control } \\
(n=15)\end{array}$ & $\begin{array}{c}\text { Stress } \\
(n=14)\end{array}$ \\
\hline Initial Anger-Hostility & $0.79(0.89)$ & $0.27(0.80)$ & $0.60(1.59)$ & $0.57(1.09)$ \\
\hline Post-test Anger-Hostility & $0.21(0.43)$ & $1.40(1.99)$ & $0.40(0.74)$ & $1.86(2.21)$ \\
\hline Initial Tension-Anxiety $^{*}$ & $5.57(2.90)$ & $5.00(2.30)$ & $5.53(2.00)$ & $4.64(2.17)$ \\
\hline Post-test Tension-Anxietya & $3.43(2.53)$ & $9.27(2.28)$ & $5.27(2.15)$ & $9.50(4.40)$ \\
\hline
\end{tabular}

Note: a Stress $>$ control, $p<.01$

\section{Pre-stress cortisol analyses}

Two participants indicated having violated the non-smoking/eating/drinking prohibition and were therefore excluded from the data set, leaving 14 women and 15 men in the stress group and 15 women and 14 men in the control group. Stress and control group did not differ with regard to pre-stress cortisol levels (means being $3.57 \mathrm{nmol} / 1$; range: 1.65 to $7.06 \mathrm{nmol} / 1$, and $4.37 \mathrm{nmol} / 1$; range: 1.30 to 10.04 nmol/1, respectively). 


\section{Cortisol stress responses}

As anticipated, the ANOVA showed a significant main effect of time $[F(3,162)=$ $6.49 ; p=.008]$ and a significant Group $\times$ Time interaction $[F(3,162)=9.76 ; p=$ $.001]$, in the absence of additional significant main or interaction effects involving sex, time, or group. The Group $\times$ Sex interaction just fell short of being significant $[F(1,54)=4.23 ; p=.054]$. Increases in cortisol levels throughout the experiment can be seen in Figure 4.1.

Men in the stress group showed a mean delta increase in salivary free cortisol of $4.61(S D=7.14) \mathrm{nmol} / \mathrm{l}$, while women showed a mean increase of $1.53(S D=$ 2.12) $\mathrm{nmol} / \mathrm{l}$. Control participants showed minor to no increase in response to the filler task, mean increases being $-0.16(S D=0.93) \mathrm{nmol} / \mathrm{l}$ for men and $0.24(S D=$ $0.85) \mathrm{nmol} / \mathrm{l}$ for women. An ANOVA on delta increases in cortisol yielded a main effect of group $[F(1,54)=9.06 ; p=.004]$. No other main or interaction effects were detected. Eleven out of 29 participants (i.e., 38 percent; 8 men and 3 women) could be classified as showing a straightforward cortisol response.

\section{Stress-induced cortisol responses}

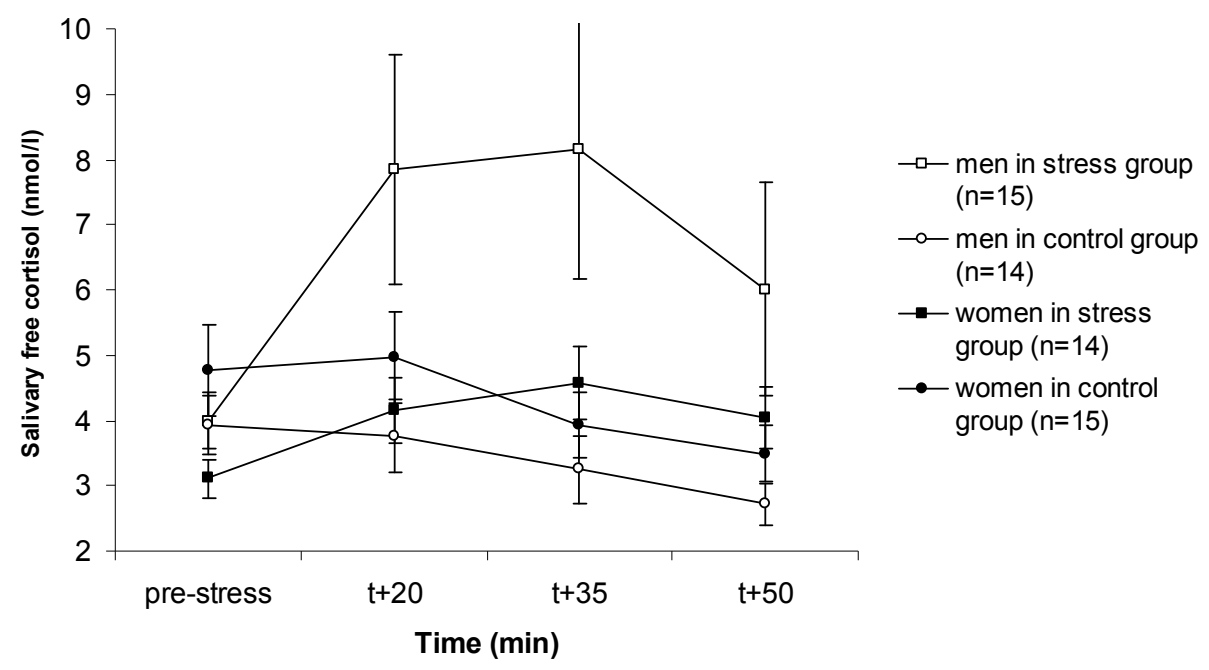

Figure 4.1. Study 1 mean free salivary cortisol levels (nmol/l) in the stress and control group for men and women separately. Data points indicate cortisol levels before stress/filler manipulation (prestress), immediately after the stress (TSST) or filler task ( $t+20), 15$ minutes after stress or filler task $(\mathrm{t}+35)$, and after testing was completed $(\mathrm{t}+50)$. 


\section{Memory performance}

Descriptive statistics for the DRM recall task and the DRM recognition task are shown in Table 4.2. ANOVA showed a main effect of sex $[F(1,54)=4.27 ; p=$ $.044]$ and a significant Group $\times$ Sex interaction $[F(1,54)=4.59 ; p=.037]$, but no main effect of group for the number of commission errors in the free recall task. Post-hoc tests showed that the interaction effect was due to men making more commission errors, but only so in the control group [ $t(27)=-2.60 ; p=.015]$. There were, however, no significant main effects or interactions involving sex or group for proportion correct recall, proportion of falsely recalled critical lure words, proportion correct recognition, or proportion of falsely recognized critical lures. Delta cortisol increases were not significantly related to false recall $(r=-.08)$ or false recognition $(r=-.23)$ rates among participants exposed to the TSST.

Table 4.2. Study 1 mean number of commission errors, proportion correct recall, and proportion false recall (i.e., critical lure words) on the free recall task and proportion correct (i.e., "old" words recognized as "old" and "new" words recognized as "new") and false (i.e., incorrectly recognizing critical lures as "old") recognition for men and women in the stress and control group. Standard deviations are given in parentheses.

\begin{tabular}{|l|c|c|c|c|}
\hline & \multicolumn{2}{|c|}{ MEN } & \multicolumn{2}{c|}{ WOMEN } \\
\cline { 1 - 5 } & $\begin{array}{c}\text { Control } \\
(n=14)\end{array}$ & $\begin{array}{c}\text { Stress } \\
(n=15)\end{array}$ & $\begin{array}{c}\text { Control } \\
(n=15)\end{array}$ & $\begin{array}{c}\text { Stress } \\
(n=14)\end{array}$ \\
\hline Number of commissions $^{\mathrm{a}}$ & $5.14(4.45)$ & $2.80(2.86)$ & $1.93(1.71)$ & $2.86(1.79)$ \\
\hline Proportion correct recall & $.69(.11)$ & $.68(.07)$ & $.70(.08)$ & $.68(.05)$ \\
\hline Proportion false recall & $.42(.18)$ & $.38(.18)$ & $.32(.19)$ & $.41(.19)$ \\
\hline Proportion correct recognition & $.76(.04)$ & $.75(.05)$ & $.73(.05)$ & $.76(.02)$ \\
\hline Proportion false recognition & $.69(.25)$ & $.62(.28)$ & $.61(.25)$ & $.70(.22)$ \\
\hline
\end{tabular}

Note: ${ }^{a}$ Men in control group $>$ men in stress group, $p<.05$

\section{Discussion}

The main results of study 1 can be summarized as follows. First, we replicated the standard effect obtained in numerous other DRM studies (e.g., Roediger \& McDermott, 1995; Roediger et al., 1996; Stadler, Roediger, \& McDermott, 1999) in that both participants in the stress and the control group exhibited false recall and/or recognition of the critical lure. Second, false recall and false recognition rates did not differ between the stress and the control group. To some extent, this latter finding contradicts previous work of Payne et al. (2002), who found elevated false recognition rates in participants exposed to the TSST. One possible explanation for these divergent findings is that in our study stress responses in terms of 
cortisol remained at the low end of the continuum. It may well be that false recollections in a DRM paradigm are heightened only when cortisol levels are substantially elevated. Indeed, studies using acute psychosocial stressors like the TSST to activate the hypothalamus-pituitary-adrenal (HPA) axis have noted differential effects of stress on memory performance for high and low cortisol responders (e.g., Elzinga \& Roelofs, 2005; Takahashi et al., 2004). Also worthy of note is that Kirschbaum, Kudielka, Gaab, Schommer, and Hellhammer (1999) found that for women, phase of menstrual cycle plays an important role in the responsiveness to acute psychosocial stressors. No attempt was made to control for menstrual cycle in study 1 . Keeping in mind the limitations of study 1 we conducted a second study that sought to further delineate the effects of acute cortisol responses on rates of false recall and recognition in a DRM paradigm.

\section{STUDY 2}

Study 2 increased the sample size so as to include 34 men and 34 women in the stress group. This gave us the opportunity to differentiate between high cortisol and low cortisol responders whilst maintaining a reasonable number of participants per group. Furthermore, only women in their late luteal phase of the menstrual cycle were included in study 2.

\section{Methods}

\section{Participants}

Ninety-two healthy undergraduate students (46 men and 46 women) participated in study 2 . Their mean age was 19.74 years $(S D=1.87)$. Participants were excluded in case they suffered from any cardiovascular diseases, endocrine disorders, or asthma, or used any medication. All test protocols were approved by the standing ethics committee of the Psychology Faculty of Maastricht University. All participants signed a written informed consent and were given a small financial compensation (10 euro; approximately 12.5 U.S. dollars) for completing the experiment. As cortisol responses are influenced by menstrual cycle, only women who selfreportedly were in their luteal phase (i.e., days 21-25 of the menstrual cycle) were included. During the late luteal phase stress-induced cortisol responses of women appear to be similar to those of men (Kirschbaum et al., 1999).

\section{Materials}

Materials (i.e., POMS and TSST) used were the same as those employed in study 1. The DRM task differed somewhat from study 1 in that study 2 relied on 8 rather 
than 12 DRM lists, which had to do with practical considerations. A 48-item recognition task was construed analogous to the recognition task used in study 1. Saliva sampling and biochemical analyses were performed similar to those in study 1.

\section{Design}

Participants were assigned to one of two groups. Thirty-four men and 34 women served as the stress group and underwent the TSST, while 12 men and 12 women were assigned to a control group that included a filler task. The two groups did not differ with respect to age (stress group $M=19.69$ years, $S D=1.75$; control group $M=19.87$ years, $S D=2.23$ ). Thus, as was the case in study 1 , study 2 was designed according to a 2 (group: stress vs. control) $\times 2$ (sex: men vs. women) set-up.

\section{Procedure}

Study 2 basically followed the procedure of study 1 (cf. supra). In study 2, the filler task consisted of reading a neutral text. The POMS was administered only once (i.e., after the TSST or filler task). All memory measures were completed after about 40 minutes and total time of the entire test session never exceeded 50 minutes.

\section{Statistical analyses}

A 2 (group) $\times 2$ (sex) ANOVA was used to evaluate feelings of subjective stress (POMS). Cortisol levels were analyzed as in study 1. Delta cortisol increases were computed and analyzed analogous to study 1 . We then looked at high responders (i.e., a delta increase in cortisol $>2.50 \mathrm{nmol} / \mathrm{l}$; see study 1) versus low responders versus controls. For each dependent measure, a 3(responder group: high responders vs. low responders vs. controls) $\times 2$ (sex) ANOVA was employed. Spearman rho correlations between false recall and recognition rates and delta cortisol increases were calculated for participants in the stress group. Greenhouse-Geisser correction was used where appropriate. Alpha was set at .05 and adjusted (Bonferroni) for multiple comparisons where necessary.

\section{Results}

\section{Manipulation check}

Mean scores on the Anger-Hostility and Tension-Anxiety POMS subscales indicating levels of subjective stress are shown in Table 4.3. Main effects of group were found for the Anger-Hostility $[F(1,88)=16.11 ; p<.001]$ as well as the TensionAnxiety $[F(1,88)=26.42 ; p<.001]$ subscale scores, with participants in the stress group indicating feeling more stressed than their control counterparts. For both 
Anger-Hostility as well as Tension-Anxiety, no other main or interaction effects were found.

Table 4.3. Study 2 sum scores on subscales Anger-Hostility and Tension-Anxiety of the Profile of Mood States for men and women separately. Standard deviations are given in parentheses.

\begin{tabular}{|l|c|c|c|c|}
\hline & \multicolumn{2}{|c|}{ MEN } & \multicolumn{2}{c|}{ WOMEN } \\
\hline & $\begin{array}{c}\text { Control } \\
(n=12)\end{array}$ & $\begin{array}{c}\text { Stress } \\
(n=34)\end{array}$ & $\begin{array}{c}\text { Control } \\
(n=12)\end{array}$ & $\begin{array}{c}\text { Stress } \\
(n=34)\end{array}$ \\
\hline Anger-Hostilitya & $0.17(0.39)$ & $3.53(3.87)$ & $0.25(0.62)$ & $2.32(2.56)$ \\
\hline Tension-Anxietya & $3.25(1.81)$ & $8.12(3.22)$ & $5.08(2.23)$ & $7.68(3.44)$ \\
\hline
\end{tabular}

Note: ${ }^{\text {S Stress }}>$ control, $p<.01$

\section{Pre-stress cortisol analyses}

None of the 92 participants indicated having violated the non-smoking/eating/ drinking exclusion criteria. There were no differences between the stress and control group with regard to pre-stress cortisol levels, means being $7.40 \mathrm{nmol} / 1$ (range: 1.76 to $25.44 \mathrm{nmol} / \mathrm{l}$ ) and $5.86 \mathrm{nmol} / 1$ (range: 2.65 to $25.51 \mathrm{nmol} / \mathrm{l}$ ), respectively.

\section{Cortisol stress responses}

As expected, we found significant main effects of time $[F(3,264)=7.49 ; p=.001]$ and group $[F(1,88)=7.02 ; p=.01]$, as well as a significant Group $\times$ Time interaction $[F(3,264)=5.94 ; p=.002]$. No other main or interaction effects involving sex, time, or group were observed.

Delta increases in cortisol differed significantly between groups for men as well as women, as indicated by a main effect of group $[F(1,88)=11.05 ; p=.001]$ in the absence of additional main or interactive effects. Because of the high variance in cortisol responses to the TSST, a post-hoc split between high and low cortisol responders was conducted. Twenty-one of all men and 13 of all women exposed to the TSST responded with a clear-cut cortisol response. Mean delta increases for high $(n=34)$ and low $(n=34)$ responders and controls can be found in Table 4.4. As anticipated, an ANOVA confirmed that delta responses differed significantly between responder groups $[F(1,86)=78.06 ; p<.001]$, while no other main or interactive effects were found. Post-hoc tests on delta increases proved that high responders showed larger increases than low responders and controls $(p$ $<.001)$. Low responders, however, did not differ from controls. Figure 4.2 shows increases in cortisol levels throughout the experiment for male and female high responders, low responders, and controls. 
Table 4.4. Study 2 mean delta cortisol responses (nmol/l) for high responders, low responders, and controls for men and women separately. Standard deviations and sample sizes are given in parentheses.

\begin{tabular}{|c|c|c|}
\hline & Men & Women \\
\hline High cortisol responders ${ }^{\mathrm{a}, \mathrm{b}}$ & $8.82(3.97 ; n=21)$ & $8.52(4.92 ; n=13)$ \\
\hline Low cortisol responders ${ }^{\mathrm{a}}$ & $-0.23(1.96 ; n=13)$ & $-0.28(2.69 ; n=21)$ \\
\hline Controls $^{b}$ & $0.44(2.50 ; n=12)$ & $0.25(0.96 ; n=12)$ \\
\hline
\end{tabular}

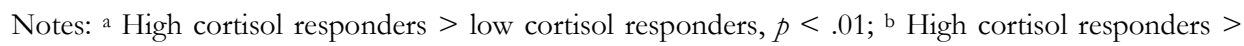
controls, $p<.01$

\section{Memory performance ${ }^{13}$}

Table 4.5 gives descriptive results from the DRM recall and DRM recognition task. For the number of commission errors, ANOVA showed a main effect of sex $[F(1,86)=7.11 ; p=.009]$ devoid of other main or interactive effects. As can be seen in Table 4.5, this had to do with men committing more errors of commission than women. An ANOVA performed on correct recall showed a significant main effect of responder group $[F(2,86)=4.48 ; p=.014]$, while no other main or interaction effect was found. Post-hoc tests showed that controls showed superior correct free recall performance in comparison to low $[t(56)=2.18 ; p=.034]$ and high $[t(56)=3.07 ; p=.003]$ cortisol responders. There were, however, no differences between low and high responders. For falsely recalled critical lures, proportion correct recognition, and false recognition (i.e., recognizing a critical lure as "old") rates, no significant main or interactive effects were found.

Delta increases were not significantly related to false recall $(r=.02)$ or false recognition $(r=.08)$ rates among participants exposed to the TSST. Even when data from study 1 and study 2 was collapsed and then correlations were computed, these $r^{2}$ s remained non-significant $(n=97 ; r=-.02$ and $r=.02$ for false recall and recognition, respectively).

\footnotetext{
${ }^{13}$ Note that in this case, a median split would result in the same high and low responder groups. To check whether other ways to split the present data would lead to similar results, we also conducted a tertile split. For each of the dependent variables, 4(responder group: high responders; $n=23$ vs. medium responders; $n=22$ vs. low responders; $n=23$ vs. controls; $n=24) \times 2$ (sex) ANOVA's yielded highly similar results, with the only significant results being an effect of $\operatorname{sex}[F(3,84)=6.35 ; p=.014]$ for number of commission errors and a main effect of responder group for correct recall $[F(3,84)=$ $2.99 ; p=.036]$.
} 


\section{Stress-induced cortisol responses}

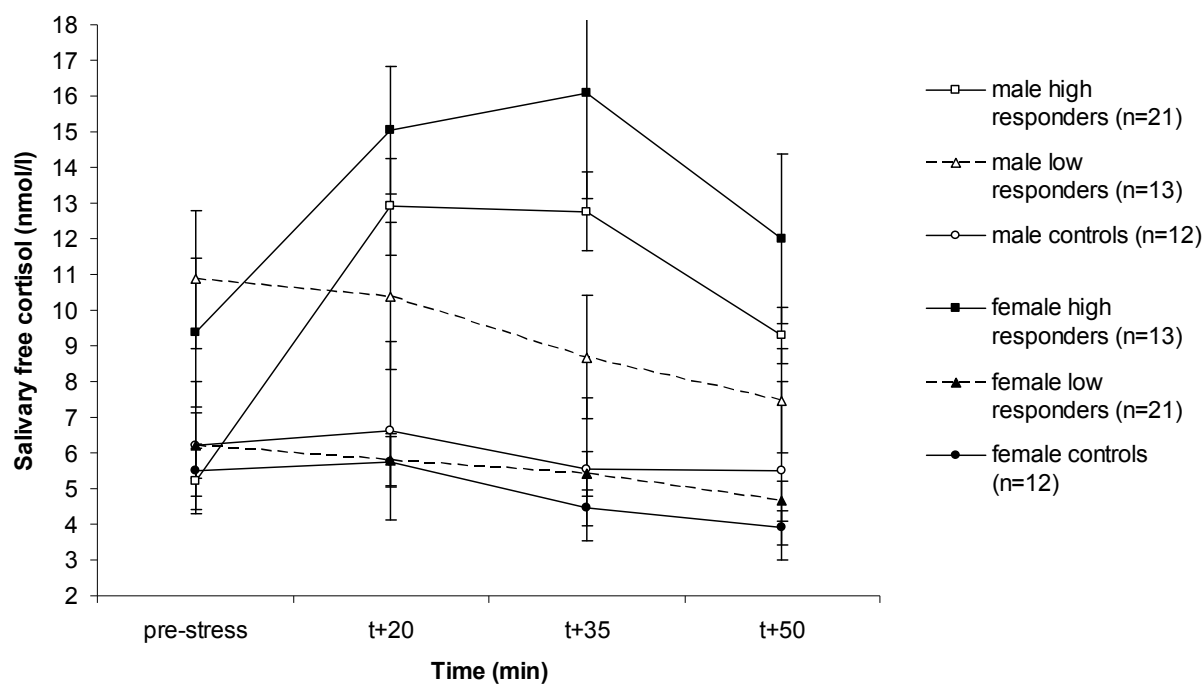

Figure 4.2. Study 2 mean free salivary cortisol levels (nmol/l) male and female high responders, male and female low responders, and male and female controls. Data points indicate cortisol levels before stress/control manipulation (pre-stress), immediately after the stress (TSST) or filler task ( $t+20), 15$ minutes after stress or filler task $(t+35)$, and after testing was completed $(t+50)$.

Table 4.5. Study 2 mean number of commission errors, proportion correct recall, and proportion false recall (i.e., critical lure words) on the free recall task, and proportion correct (i.e., "old" words recognized as "old" and "new" words recognized as "new") and false (i.e., incorrectly recognizing critical lures as "old") recognition. Descriptive results are provided for male and female high responders, low responders, and controls separately. Standard deviations are given in parentheses.

\begin{tabular}{|l|l|l|l|l|c|c|}
\hline & \multicolumn{3}{|c|}{ MEN } & \multicolumn{3}{|c|}{ WOMEN } \\
\hline & $\begin{array}{c}\text { Controls } \\
(n=12)\end{array}$ & $\begin{array}{c}\text { Low re- } \\
\text { sponders } \\
(n=13)\end{array}$ & $\begin{array}{c}\text { High re- } \\
\text { sponders } \\
(n=21)\end{array}$ & $\begin{array}{c}\text { Controls } \\
(n=12)\end{array}$ & $\begin{array}{c}\text { Low re- } \\
\text { sponders } \\
(n=21)\end{array}$ & $\begin{array}{c}\text { High re- } \\
\text { sponders } \\
(n=13)\end{array}$ \\
\hline \begin{tabular}{l|l|c|c|c|c|c|} 
Number of commis- \\
sions $^{\mathrm{a}}$
\end{tabular} & $2.75(2.38)$ & $\begin{array}{c}2.77 \\
(1.17)\end{array}$ & $\begin{array}{c}3.43 \\
(3.01)\end{array}$ & $\begin{array}{c}1.00 \\
(0.74)\end{array}$ & $\begin{array}{c}2.62 \\
(1.66)\end{array}$ & $1.92(1.19)$ \\
\hline $\begin{array}{l}\text { Proportion correct } \\
\text { recallb,c }\end{array}$ & $.71(.11)$ & $.65(.07)$ & $.62(.05)$ & $.71(.07)$ & $.67(.07)$ & $.68(.08)$ \\
\hline Proportion false recall & $.39(.23)$ & $.34(.16)$ & $.35(.21)$ & $.27(.21)$ & $.31(.21)$ & $.37(.18)$ \\
\hline $\begin{array}{l}\text { Proportion correct } \\
\text { recognition }\end{array}$ & $.91(.08)$ & $.86(.07)$ & $.87(.07)$ & $.88(.07)$ & $.88(.05)$ & $.89(.07)$ \\
\hline $\begin{array}{l}\text { Proportion false rec- } \\
\text { ognition }\end{array}$ & $.68(.32)$ & $.65(.21)$ & $.70(.30)$ & $.57(.26)$ & $.71(.17)$ & $.67(.24)$ \\
\hline
\end{tabular}

Notes: a Men $>$ women, $p<.01$; b Controls $>$ high cortisol responders, $p<.01$; c Controls $>$ low cortisol responders, $p<.01$ 


\section{Discussion}

The main results of study 2 can be summarized as follows. To begin with, there were significant differences between high cortisol responders, low cortisol responders, and controls with respect to correct recall of presented words. More specifically, exposure to the TSST resulted in impairments in correct free recall in comparison to control participants. High cortisol responders, however, did not differ from low cortisol responders in correct free recall rate. Neither did we find evidence for the idea that the effect of stress on memory is modulated by sex, although overall men made more commission errors than women. Taken together, our data fit well with studies demonstrating the undermining effects of acute stress on recall of neutral words (e.g., Jelicic, Geraerts, Merckelbach, \& Guerrieri, 2004; Kirschbaum et al., 1996b; Tops et al., 2003). Second, despite its undermining effect on correct free recall exposure to the TSST did not result in heightened levels of false recall of the critical lures. This was true for low as well as for high cortisol responders. Moreover, false recognition rates also did not differ between low or high cortisol responders and controls.

\section{General Discussion}

To summarize, participants in both study 1 and study 2 falsely recalled and recognized the non-presented critical lures at rates similar to those reported elsewhere (e.g., Roediger \& McDermott, 1995; Stadler et al., 1999). Results from study 1 showed that stress and control group did not differ with respect to false recall and false recognition of critical lures. Study 2 showed that high cortisol responders did not demonstrate elevated levels of false recall or false recognition in comparison to low cortisol responders or controls. There were, however, significant impairments in correct recall of presented words for high and low cortisol responders compared to their control counterparts (study 2). No significant correlations between false recall and recognition rates and delta increases in cortisol were revealed.

The finding that stressed (study 1) and high and low cortisol responders (study 2) did not differ from controls in their rate of false recollection, to some extent contradicts the work of Payne et al. (2002). It should be noted, though, that there are substantial differences in methodology between the Payne et al. study and the current studies. That is, the present studies drew upon a free recall task following each DRM list and an overall recognition task after all DRM lists had been presented, while the Payne et al. study employed a single recognition task subsequent to each DRM list.

A recent meta-analysis by Het et al. (2005; p. 780) looking at administered acute cortisol and memory performance concluded that for recognition memory 
"[...] the effect sizes were on average descriptively smaller — almost zero - than the effect sizes for free or cued recall performances. This finding may indicate that recognition memory performance is less suitable to uncover effects of cortisol on memory." Thus, assuming that acute cortisol elevations were responsible for the heightened rate of false recognition in the Payne et al. (2002) study, one would have predicted that these effects would have been even larger with recall tasks of the type used in the current studies.

Meanwhile, GCs (e.g., cortisol) may yield very different results for recall and recognition tasks as these tasks seem to tap different neural structures involved in memory (e.g., Brown \& Aggleton, 2001; Buckner \& Wheeler, 2001). Recognition performance on DRM related tasks has been investigated by means of EventRelated Potentials (e.g., Curran, Schacter, Johnson, \& Spinks, 2001; Fabiani, Stadler, \& Wessels, 2000), Positron Emission Tomography (Schacter et al., 1996), and functional Magnetic Resonance Imaging (e.g., Cabeza, Rao, Wagner, Mayer, \& Schacter, 2001). Collectively, these studies indicate that while the hippocampus, together with several cortical regions, contributes to false recognition in the DRM paradigm, rates at which stimuli are falsely recognized may be under regulation from monitoring processes of the prefrontal cortex (for a thorough review, see Schacter \& Slotnick, 2004).

Animal, but also human research shows that GCs may differentially affect the various memory phases (e.g., Roozendaal, 2002). Specifically, while GCs like cortisol have been shown to enhance memory formation (e.g., Buchanan \& Lovallo, 2001), there have also been reports of an impairing effect of GCs on retrieval (e.g., Wolf et al., 2004). Roozendaal (2002) suggests that when triggered by stress, the basolateral complex of the amygdala turns the brain into a memory-consolidation state, thereby resulting in strong consolidation for ongoing events while at the same time undermining future attempts at retrieval. This might be important given the fact that a DRM task inherently comprises only one session in which DRM lists are studied by research participants who subsequently, but during the very same session, are probed for recall and/or recognition. Indeed, from a theoretical viewpoint it is conceivable that facilitating effects of cortisol increases on memory formation might overshadow the detrimental effects of cortisol on retrieval processes.

In addition, several studies involving acute psychological stressors including, but not limited to, the TSST have shown a sex effect in HPA axis stress responses (for a comprehensive review, see Kudielka \& Kirschbaum, 2005). In particular, Kudielka and Kirschbaum (2005, p. 117), note that "[...] most psychological stress studies revealed that there are (a) no significant sex differences or (b) higher cortisol responses in young men than in young women [...]". In both study 1 and study 2 , delta cortisol increases were indeed larger for men than for women, although this difference fell short of being significant. This is especially important given that, at least in study 1, cortisol responses to the TSST were relatively small. Further- 
more, recent studies suggest that individual differences in cortisol reactivity may very well reflect genetic predispositions (e.g., Wüst, van Rossum, Federenko, Koper, Kumsta, \& Hellhammer, 2004), anticipatory cognitive appraisal (e.g., Gaab, Rohleder, Nater, \& Ehlert, 2005; Rohrmann, Hennig, \& Netter, 1999), polymorphism in sensitivity of GC receptor tissue (e.g., Rohleder, Wolf, \& Kirschbaum, 2003), or even variation in lifetime cortisol exposure (e.g., Lupien, Wilkinson, Briere, Ng Ying Kin, Meaney, \& Nair, 2002a).

In addition to animal studies, research involving humans has occasionally found evidence suggesting that sex differences may modulate the effects of GCs on memory functioning. In contrast, no differences between men and women on memory performance after exposure to the TSST were found in the present studies. However, we did observe that relatively independent of stress, men produced more commissions (i.e., confabulated extra-list words) on the free recall task. Another factor that might be of relevance when studying the effects of cortisol on memory performance is that cortisol levels in the morning appear to lead to impairing effects, while generally yielding no or slightly enhancing effects when tested in the afternoon (Lupien, Wilkinson, Briere, Menard, Ng Ying Kin, \& Nair, 2002b). From their meta-analysis, Het et al. (2005) concluded that studies performed in the afternoon on average yielded an effect size that was smaller and in the opposite direction than the effect size found by studies performed in the morning. Therefore, it awaits to be seen whether our failure to detect an effect of cortisol increase on false recall can be generalized to other times of day.

Some notes on the methodological limitations of the present studies are in order. First, the current studies used only a limited number of cortisol measurements to characterize the stress responses. Hence, it is possible that we missed the peak level of cortisol in a substantial number of our participants. Secondly, it can be argued that our studies used only a single session memory performance task, which makes it impossible to differentiate between learning and recall effects following acute psychosocial stress. Thus, it cannot be excluded that beneficial effects of cortisol on memory formation were reversed through the impairing effect of cortisol on memory retrieval resulting in a net effect that did not differ from controls. Also, it must be acknowledged here that, especially in study 1, cortisol responses to the TSST were not as large as in some other studies (e.g., Kirschbaum et al., 1992; but see Elzinga \& Roelofs, 2005, for similar cortisol responses among high and low responders to the TSST). Future research should take these limitations into account.

In sum, then, our results suggest that cortisol elevations alone might be insufficient to increase false recall or recognition rates in a DRM paradigm. The fact that heightened false recognition rates following acute stress do not seem strongly related to cortisol responses or to a modulating effect of sex indicates that factors other than HPA axis responses may be responsible for the detrimental effects of acute stress on false recollections reported elsewhere (e.g., Payne et al., 2002). A 
likely candidate for this would be the brain's noradrenergic system that controls autonomic output (Sved, Cano, Passerin, \& Rabin, 2002). Clearly, this issue warrants further study. What can be said with some confidence is that in the present studies, exposure to an acute psychosocial stressor - even when this reduced correct recall - did not necessarily promote false recall and recognition. 


\section{Enhanced memory performance on an internal-internal source monitoring test following acute psychosocial stress}

This chapter is an adapted version of: Smeets, T., Jelicic, M., Merckelbach, H., Peters, M., Fett, A., Taverniers, J., Henquet, C., \& Dautzenberg, J. (in press). Enhanced memory performance on an internal-internal source monitoring test following acute psychosocial stress. Behavioral Neuroscience. 


\section{Summary}

Research on the effect of acute stress and high levels of glucocorticoids on memory have largely focused on memory tasks involving the medial temporal lobe (e.g., declarative memory). Less is known, however, about the effects of stress and glucocorticoids on more strategic memory processes regulated by the prefrontal cortex (e.g., source monitoring). The current study investigated whether exposure to acute psychosocial stress would result in altered source monitoring performance relative to a non-stressed control group. To this end, non-smoking, healthy young men were assigned to either a stress $(n=22)$ or a control $(n=18)$ condition after which they were given an internal source monitoring test. Results show that relative to controls, stressed participants made fewer source monitoring errors. This study suggests that stress may have differential effects on memory depending on whether the memory test is regulated by the prefrontal cortex or the medial temporal lobe.

\section{Introduction}

Source monitoring refers to cognitive processes that are involved in making attributions about the origins of memories, knowledge and beliefs (Johnson, Hashtroudi, \& Lindsay, 1993). Consistent with the source monitoring framework proposed by Johnson and co-workers (1993), three major types of source monitoring can be distinguished. Reality monitoring refers to the ability to discriminate between internally generated memories versus externally generated memories (e.g., "Did I hear that on the news or did I imagine that?"). Alternatively, distinguishing between memories of two externally derived sources is termed external source monitoring (e.g., "Did I hear that on the news or did Tim tell me that?"). Finally, internal source monitoring is said to occur when people have to discriminate between two internally generated memories ("Did I say that out loud or did I merely think that?"). Inaccuracies in judging the source of a memory (i.e., source monitoring errors) are by no means uncommon and can have important ramifications, as evidenced by mistaken eyewitness testimonies in which fragments of real experiences are accurately recalled, but are attributed to the wrong person, time, or location (e.g., Ross, Ceci, Dunning, \& Toglia, 1994).

Accurate source monitoring is dependent on cognitive processes that initially bind features into complex memories and on processes that reactivate and evaluate such features (Johnson et al., 1993). Brain areas involved in these cognitive processes include the medial temporal regions, which are essential for binding and reactivation, and frontal regions, particularly lateral frontal regions, that are of special importance for strategic retrieval and/or evaluation of features of memories (John- 
son et al., 1993; Moscovitch, 1994). Evidence for the vital role of the prefrontal cortex (PFC) in source monitoring comes from clinical studies involving patients with frontal lobe damage (e.g., Janowsky, Shimamura, \& Squire, 1989; Schacter, Harbluk, \& McLachlan, 1984) and, more recently, studies using neuroimaging techniques (e.g., Dobbins, Foley, Schacter, \& Wagner, 2002; Dobbins, Rice, Wagner, \& Schacter, 2003; Mitchell, Johnson, Raye, \& Greene, 2004; Nolde, Johnson, \& D'Esposito, 1998; Ranganath, Johnson, \& D'Esposito, 2000; Raye, Johnson, Mitchell, Nolde, \& D'Esposito, 2000; Rugg, Fletcher, Chua, \& Dolan, 1999; Slotnick, Moo, Segal, \& Hart, 2003). On the basis of this type of empirical evidence, Johnson and Raye (1998, 2000; see also Nolde et al., 1998) suggested that the right PFC supports heuristic processing (e.g., item recognition), whereas the left PFC (possibly together with the right PFC) subserves source monitoring.

A vast amount of animal (e.g., McGaugh \& Roozendaal, 2002; Roozendaal; 2000) and human (for reviews, see Het, Ramlow, \& Wolf, 2005; Lupien \& Lepage, 2001; Wolf, 2003) research has shown that glucocorticoid (GC) secretion from the adrenal cortex during stressful episodes may have facilitating as well as disruptive effects on memory formation, consolidation, and retrieval. Research suggests that memory facilitation may occur when GC receptors are moderately stimulated while at the same time high affinity mineralocorticoid receptors are fully saturated. In contrast, when GC receptors are extremely occupied under stressful circumstances, high GC levels may exert detrimental effects on memory (e.g., de Kloet, Oitzl, \& Joëls, 1999; Oitzl \& de Kloet, 1992; Reul \& de Kloet, 1985). Moreover, it seems that adrenergic activation of the basolateral complex of the amygdala (BLA) and the hippocampus is a prerequisite for GCs to impair retrieval (e.g., Roozendaal, Hahn, Nathan, de Quervain, \& McGaugh, 2004). Together with brain regions such as the PFC and the hippocampus, activation of the BLA along with effects of emotional arousal can mediate the effects of GCs on memory (e.g., McGaugh \& Roozendaal, 2002).

In a series of research papers, Moscovitch and colleagues have convincingly argued that medial temporal lobe (MTL) contributions to memory are distinct from PFC involvement in memory (e.g., Moscovitch, 1992; Moscovitch \& Winocur, 1992; for a recent review, see Moscovitch \& Winocur, 2002). These authors assert that although there is no question as to its importance to memory processes, the MTL is primarily concerned with the content of memories. The frontal cortex, on the other hand, operates on the input and output of MTL in encoding and in performing heuristic and strategic operations, e.g., determining the source of a memory (Johnson et al., 1993; Moscovitch \& Winocur, 2002). Whereas the effects of acute stress and high GC levels on memory recollection involving the MTL have been well-studied, their effects on more strategic memory processes (e.g., retrieval cue generation and specification, verification, source monitoring) 
that are under the regulation of the PFC remain largely unknown. ${ }^{14}$ Interestingly, animal studies have shown that the PFC is a significant target for negative feedback actions of GCs and that chronic GC administration and behavioural stress can result in dendritic reorganization in the medial PFC (e.g., Charney, 2004; Radley et al., 2004; Sanchez, Young, Plotsky, \& Insel, 2000). In conjunction with other findings implicating the PFC as a target for circulating GCs (e.g., Lupien \& Lepage, 2001), this suggests that high levels of circulating GCs may stimulate PFC regulated source memory.

The present study investigated whether exposure to an acute psychosocial stressor (i.e., the Trier Social Stress Test; see Kirschbaum, Pirke, \& Hellhammer, 1993) and/or the consequential cortisol elevations would result in altered source monitoring performance relative to a non-stressed control group. If acute psychosocial stress and high GC stress responses affect PFC regulated source monitoring performance similar to MTL mediated memory processes, one would expect that stressed participants would exhibit more source monitoring errors than nonstressed control participants. On the other hand, given that there are studies showing increased cerebral blood flow in the right PFC during psychological stress (e.g., Wang et al., 2005), one could speculate that acute stress and/or high levels of GCs have enhancing effects on source memory performance. Note, however, that source monitoring appears to be mainly lateralized to the left PFC, and that increased blood flow in the right PFC may instead have impairing effects by depleting the resources that are necessary for accurate source monitoring.

\section{Methods}

\section{Participants}

Our sample consisted of 40 young healthy undergraduate students. Participants were non-smokers with a normal Body Mass Index (BMI), and all were native Dutch speakers. To rule out that sex differences could play a confounding role in cortisol reactions to the stress task (for a review, see Kudielka \& Kirschbaum, 2005), only men were included in the present study. Their mean age was 19.2 years $(S D=1.4)$. Participants were excluded from the study in case they suffered from cardiovascular diseases, endocrine disorders, or were on any kind of medication. Test protocols were approved by the standing ethics committee of the Psychology Faculty of Maastricht University. All participants signed a written informed consent and were given a small financial compensation (12.5 euro; approximately 15 U.S. dollars) for completing the experiment.

\footnotetext{
14 One important exception to this is work on the link between stress and working memory (for examples, see al' Absi, Hugdahl, \& Lovallo, 2002; Elzinga \& Roelofs, 2005; Lupien, Gillin, \& Hauger, 1999).
} 


\section{Materials}

Several personality questionnaires, including the Thought-Action Fusion (TAF) scale (Shafran, Thordarson, \& Rachman, 1996), the Creative Experiences Questionnaire (CEQ; Merckelbach, Horselenberg, \& Muris, 2001) and the Community Assessment of Psychic Experiences (Stefanis et al., 2002) trait (CAPE-T) and state (CAPE-S) version, were administered as filler tasks. Psychometric data derived from these questionnaires will not be addressed here.

Trier Social Stress Test (TSST). The TSST (Kirschbaum et al., 1993) is a psychosocial challenge test that basically consists of a 10 minute preparation period, a 5 minute free speech, and a 5 minute mental arithmetic task in front of an audience while being videotaped. The TSST is a valid and reliable procedure to induce physiological stress responses in children and young as well as elderly adults (e.g., Kirschbaum, Wüst, \& Hellhammer, 1992; Kudielka, Buske-Kirschbaum, Hellhammer, \& Kirschbaum, 2004a; Kudielka, Schommer, Hellhammer, \& Kirschbaum, 2004b). In a recent meta-analysis, the TSST was found to provoke the most robust physiological stress responses (i.e., cortisol stress responses) relative to various other laboratory stress tasks (Dickerson \& Kemeny, 2004). Though the original TSST protocol has served our lab well for eliciting stress responses (e.g., Smeets, Jelicic, \& Merckelbach, 2006a, 2006b), the current study used a personalized TSST. In this modified version of the TSST, participants were asked to describe their personality characteristics in front of an audience in a foreign language (i.e., in English) over a period of 10 minutes while standing, preceded by a 5 minute preparatory period. During the final 5 minutes, they were asked to solve 30 mathematical problems of above-average difficulty. For each problem, they were given a maximum of $8 \mathrm{sec}-$ onds.

Source Monitoring Test (SMT). The current study's source monitoring test was based on Parks' (1997) study and was developed to determine participants' ability to discriminate mere thoughts from actually verbalized thoughts. That is, participants had to indicate whether they had really verbalized or only imaged answers to earlier presented questions. This test has been proven to be sensitive to detecting differences in source monitoring abilities (see Henquet, Krabbendam, Dautzenberg, Jolles, \& Merckelbach, 2005). SMT materials consisted of 24 non-intrusive questions concerning personal history (e.g., "When were you born?") and opinion (e.g., "What foods do you like?"). Questions were presented on a computer screen, using a computer program specifically developed for this purpose. The sourcemonitoring test involved 16 trials. On half of the trials, single questions were presented. On the other half, questions were presented in pairs, with one question being located at the top half of the computer screen and the other being located at the bottom half of the screen. Questions were presented, after which a white screen was shown for 3 seconds. Participants had to prepare an answer to the presented questions. On single question trials, the word "answer" appeared 1 second after presentation of the white screen. On dual question trials, either the words 
"answer top" or "answer bottom" appeared. Thus, here participants had to prepare an answer to both questions, but they verbalized only one answer. This resulted in verbalizing answers to 16 questions, whereas 8 answers were covertly prepared but never verbalized. Single questions and pairs were presented in a quasi-random order, and two parallel versions were used that were counterbalanced across participants. An experimenter was present to monitor whether participants actually verbalized the answers. All participants were capable of answering the questions, indicating that the questions were simple and direct. Participants were instructed to indicate when they had prepared an answer for the questions. Preparation time varied between participants but never took longer than 10 seconds. Given this constellation, we have every reason to believe that participants actually did prepare answers during the preparation phase of two-question trials, as opposed to merely remembering the questions. Following the presentation of the questions (i.e., Source Memory Acquisition; SMA) and exposure to the TSST or filler task, participants were given a surprise recognition and source monitoring test (i.e., $\mathrm{R}+\mathrm{SMT}$ ). In this test, participants saw original questions, each paired with a new question of the same content and form. For example, the old item "When were you born?" was presented along with the new item "Where were you born?". Participants were asked to identify for each of the 24 pairs of old and new items the question they had seen before (i.e., the recognition memory aspect of the test). Further, they had to indicate whether they verbalized answers to the "old" items or only thought about an answer (i.e., the source memory aspect of the test).

\section{Saliva sampling and biochemical analyses}

Cortisol data were obtained using cotton Salivettes (Sarstedt ${ }^{\circledR}$, Nümbrecht, Germany). The uncentrifuged saliva samples were stored at $-40^{\circ}$ Celsius immediately upon collection. Salivary free cortisol levels were determined in duplicate by direct radioimmunoassay (University of Liège, Belgium), including a competition reaction between 125iodohistamine-cortisol and anti-cortisol serum made against the 3CMO-BSA conjugate. After overnight incubation at $4^{\circ}$ Celsius of $100 \mu \mathrm{l}$ of saliva, separation of free and antibody-bound 125iodohistamine-cortisol was performed via a conventional 'second antibody' method. In order to reduce sources of variability, all 7 samples taken from each participant (see below) were analyzed in the same assay. Two Salivettes contained insufficient saliva for analyses and were treated as missing values. Mean intra- and inter-assay coefficients of variation were less than 4.5 percent and 8.5 percent, respectively.

\section{Design}

Participants were quasi-randomly assigned to one of two groups. Participants were exposed to a modified version of the TSST (Kirschbaum et al., 1993) serving as the stress group $(n=22)$, whereas others were assigned to a control group that included a filler task $(n=18)$. The two groups did not differ with respect to age 
(stress group $M=19.3$ years, $S D=1.6$; control group $M=19.0$ years, $S D=1.0$ ) $[t(38)=0.73 ; p=.47]$.

\section{Procedure}

All participants were tested individually in experimental sessions run between $09.00 \mathrm{~h}$ and $12.00 \mathrm{~h}$. To allow for objective controlled cortisol sampling, all participants were deprived of food, drinks, and heavy exercise at least 1 hour prior to the test phase. After arrival in the laboratory, participants were given a resting phase of 20 minutes during which they signed a consent form and completed the CAPE-T. Subsequently, participants performed the source memory acquisition test (SMA). All participants then took part in either the modified version of the TSST, or received a filler task (i.e., reading a neutral text) of equal duration (see Figure 5.1). Afterwards, participants filled out the CEQ and completed the surprise recognition and source monitoring test (i.e., R+SMT). Finally, during a 10 minute recovery period participants completed the CAPE-S and the TAF. Cortisol samples were obtained over a 60 minute period at 7 measurement points (i.e., $-20,-10,0,+10$, $+20,+30$, and +40 minutes with reference to the onset of the stressor or filler task; see also Figure 5.1). The entire test session never exceeded 1 hour. Participants were debriefed, paid, and thanked for their participation.

\section{Statistical analyses}

Cortisol responses were analyzed using a 2 (group: stress vs. controls) $\times 7$ (time: $\mathrm{t}-20$ vs. $\mathrm{t}-10$ vs. $\mathrm{t} 0$ vs. $\mathrm{t}+10$ vs. $\mathrm{t}+20$ vs. $\mathrm{t}+30$ vs. $\mathrm{t}+40)$ Analysis of Variance (ANOVA), with the last factor being a repeated measure. For each participant individually, we computed a cortisol response (i.e., delta increase in cortisol) defined as peak cortisol level $(\mathrm{t}+10, \mathrm{t}+20, \mathrm{t}+30$, or $\mathrm{t}+40)$ after the TSST or filler task minus pre-stress cortisol level $(\mathrm{t}-10)$. Note that $\mathrm{t}-10$ rather than $\mathrm{t}-20$ was used as pre-stress cortisol level because the novelty of the first cortisol sampling procedure may have amplified the $t-20$ reading. Delta responses were analyzed using an independent samples $t$-test. Performance on the $\mathrm{R}+\mathrm{SMT}$ was analyzed following Corwin's (1994) Two-High Threshold theory. First, false-alarm rates (i.e., the number of erroneous claims of having verbalized an answer that in fact was covertly prepared) and missing rates (i.e., the number of mistaken claims of having covertly prepared an answer that in fact was verbalized) were calculated. Second, in line with Brébion, Smith, and Widlocher (1997), measures of accurate and biased discrimination between internal and external thoughts (i.e., a discrimination index and a response bias, respectively) were determined. Discrimination index was defined as: [(number of hits +0.5$) /$ (number of targets +0.1$)]-[$ (number of false alarms +0.5$) /($ number of distracters +1$)]$. Response bias was defined as: $[[$ (number of false alarms +0.5$) /$ (number of distracters +1$)] /(1$-discrimination index)]. A recognition memory score was calculated by summing the number of correctly identified old items divided by the number of items (i.e., proportion cor- 
rect recognition of memory items presented during the SMA). Finally, a total source memory score was obtained by summing the number of old memory items that participants correctly classified as verbalized or covertly prepared. To correct for misses, total source memory scores were transformed into proportions of the total number of the 16 questions minus missed items (i.e., proportion of total correct source monitoring performance). For each of the R+SMT parameters, an independent samples (group: stress vs. controls) $t$-test was conducted. To evaluate the role of cortisol in acute stress effects on source monitoring indices, low and high cortisol responder groups were defined by a median split (see below). Independent samples $t$-tests were then used to check whether low cortisol responders differed from high cortisol responders in their source monitoring performance. Within the stress group, Spearman's rho correlations (2-tailed) between delta increases in cortisol and source monitoring indices were calculated. When sphericity assumptions were violated, Greenhouse-Geisser corrected $p$-values are reported. Alpha was set at .05 unless specified otherwise, and adjusted (Bonferroni) for multiple comparisons where necessary.

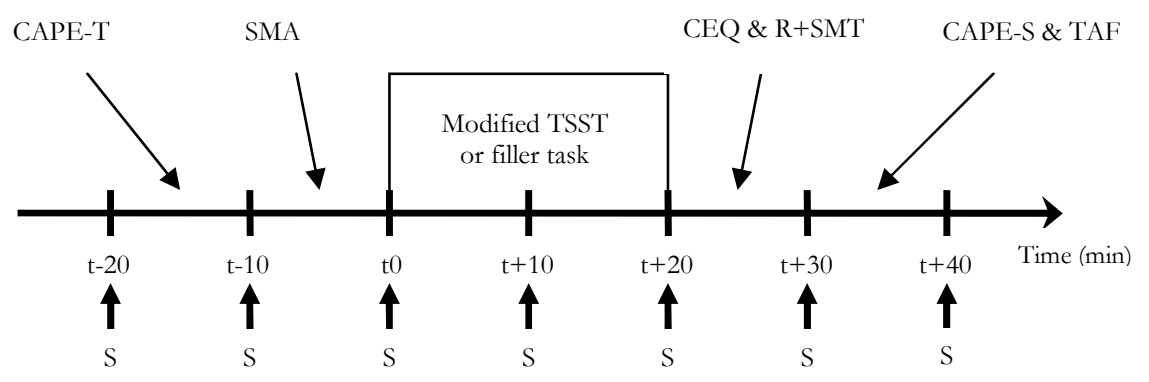

Figure 5.1. Time line of completing personality questionnaires, performing memory tasks and saliva sampling. Notes: $\mathrm{S}=$ Salivette; CAPE-T= Community Assessment of Psychic Experiences, Trait version (10 min); SMA= Source Memory Acquisition (10 min); CEQ= Creative Experiences Questionnaire (5 min); R+SMT = Recognition and Source Monitoring Test (5 min); CAPE-S= Community Assessment of Psychic Experiences, State version (8 min); TAF= Thought Action Fusion (2 min). CAPE-T, CAPE-S, TAF, and CEQ served as filler tasks.

\section{Results}

\section{Pre-stress cortisol analyses}

None of the participants self-reportedly violated the non-eating/drinking/heavy exercise prohibition. Stress and control group did not differ with regard to prestress cortisol levels at $\mathrm{t}-20, \mathrm{t}-10$, and $\mathrm{t} 0$ (all $\ell$ 's $<1.60$; all $p$ 's $>.12$ ). 


\section{Cortisol stress responses}

As expected, a significant main effect of time $[F(6,216)=6.12 ; p=.003]$ and a significant Group $\times$ Time interaction $[F(6,216)=24.39 ; p<.001]$ were found, in the absence of a main effect of group $[F(1,36)=1.32 ; p=.26]$. Delta increases in cortisol differed significantly between groups $[t(38)=-5.66 ; p<.001]$, with means of $7.47 \mathrm{nmol} / 1(S D=5.11)$ and $0.24 \mathrm{nmol} / 1(S D=1.96)$ for the stress and control group, respectively. Previous research has indicated that a cortisol increase larger than $2.5 \mathrm{nmol} / 1$ reflects a cortisol secretory episode (Van Cauter \& Refetoff, 1985) and can be taken as a clear-cut cortisol response (see, e.g., Kirschbaum et al., 1993, Kirschbaum, Wolf, May, Wippich, \& Hellhammer, 1996b; Schommer, Hellhammer, \& Kirschbaum, 2003). In our study, 18 out of 22 (i.e., 82 percent) participants in the stress group displayed a clear-cut cortisol response. Because of the high variance in cortisol responses to the TSST and following Domes, Heinrichs, Reichwald, and Hautzinger (2002; see also Elzinga \& Roelofs, 2005), a post-hoc median split between low and high cortisol responders was conducted. This resulted in a group of 11 low cortisol responders, whose mean delta increase was 3.17 ( $S D=$ 2.46) $\mathrm{nmol} / \mathrm{l}$ (i.e., a 75 percent mean increase), and a group of 11 high cortisol responders whose mean increase was $11.77(S D=2.84) \mathrm{nmol} / \mathrm{l}(+185$ percent) $[t(20)$ $=7.59 ; p<.001]$. Figure 5.2 shows increases in cortisol levels throughout the experimental session for low and high cortisol responders and non-stressed controls.

\section{Source monitoring performance}

Mean scores derived from the source monitoring test for the stress and control group can be found in Table 5.1. As can be seen, independent samples $t$-tests showed that participants in the stress group outperformed controls with regard to total source memory score, accuracy rate, proportion false positives, and the recognition memory component of the source monitoring test (all $p$ 's $<.05$ ). Groups, however, did not differ in terms of response bias or proportion false negatives (both $p$ 's $>.10$ ). To check whether these overall group differences could be mediated by the extent of the cortisol elevations, low and high cortisol responders were compared. No differences were found between low and high cortisol responders on accuracy rate (.83 vs. .85 , respectively), total source memory score (.88 vs. .88 ), proportion false positives (.13 vs. .08), recognition memory score (.99 vs. .98), response bias (.23 vs. .12), and proportion false negatives (.13 vs. .15), with all $\ell$ s $<$ 1.61 ; all $p$ 's $>.12$. Furthermore, within-stress group correlations between delta increases in cortisol and source monitoring indices remained non-significant (all $r$ 's $<$ .32 ; all $p$ 's $>.14$ ). 


\section{Stress induced cortisol responses}

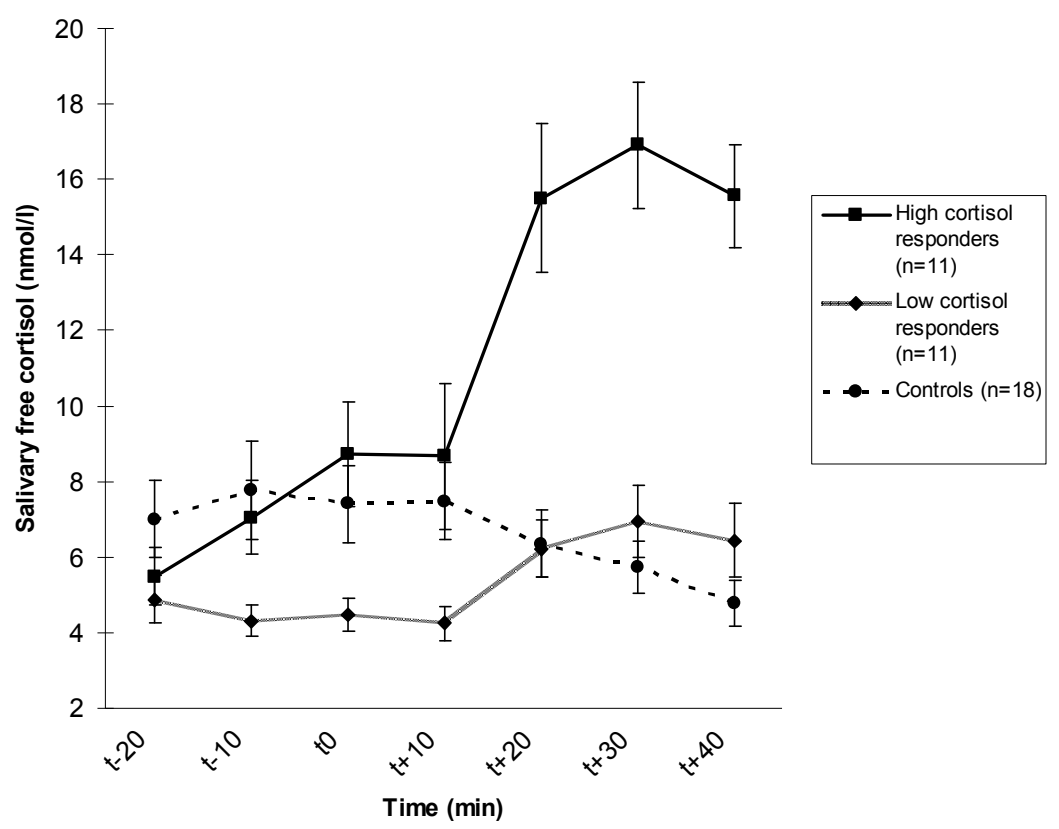

Figure 5.2. Mean free salivary cortisol levels (nmol/l) for controls and high and low cortisol responders in the stress group. Data points indicate cortisol levels throughout the session. Error bars represent standard error of mean $(S E)$.

Table 5.1. Source monitoring performance $(M \pm S D)$ of participants exposed to the TSST (stress group) or filler task (control group).

\begin{tabular}{|l|c|c|c|c|}
\hline & $\begin{array}{c}\text { Stress group } \\
(\boldsymbol{n}=\mathbf{2 2})\end{array}$ & $\begin{array}{c}\text { Control group } \\
(\boldsymbol{n}=\mathbf{1 8})\end{array}$ & $\boldsymbol{t ( 3 8 )}$ & $\boldsymbol{p}$ \\
\hline Total source memory score & $.88(.07)$ & $.80(.11)$ & -2.66 & .01 \\
\hline Discrimination index $^{\mathrm{b}}$ & $.84(.09)$ & $.72(.18)$ & -2.64 & .01 \\
\hline Response bias $^{\mathrm{c}}$ & $.17(.17)$ & $.22(.16)$ & 0.98 & .33 \\
\hline False-alarm rate $^{\mathrm{d}}$ & $.10(.10)$ & $.23(.27)$ & 2.08 & .04 \\
\hline Missing rate $^{\mathrm{e}}$ & $.14(.10)$ & $.19(.13)$ & 1.56 & .13 \\
\hline Recognition memory $^{\mathrm{f}}$ & $.99(.02)$ & $.95(.07)$ & -2.26 & .03 \\
\hline
\end{tabular}

Notes: a Proportion of total correct source attributions; ${ }^{b}$ Accuracy rate; ${ }^{c}$ Measure of biased responding; ${ }^{\mathrm{d}}$ Proportion false positives; ${ }^{\mathrm{e}}$ Proportion false negatives; ${ }^{\mathrm{f}}$ Proportion correctly recognized old items 


\section{Discussion}

The main results of the current study can be summarized as follows. Firstly, participants exposed to the stressor were better at correctly identifying verbalized and internally prepared items (i.e., total source memory score) than non-stressed controls. Secondly, relative to controls, stressed participants were more accurate at discriminating between targets and distracters when the number of errors that were made was taken into account (i.e., discrimination index). Thirdly, participants in the stress group less frequently misclassified imagined thoughts as verbalized answers (i.e., false alarms). Fourthly, these results were obtained in the absence of differences in proportion false negatives (i.e., misclassification of verbalized responses as imagined) and, importantly, without response bias playing a key role. Furthermore, relative to non-stressed controls, stressed participants were slightly better at correct recognition of previously presented items. Finally, scores derived from the R+SMT did not differ between low and high cortisol responders, suggesting that the magnitude of the cortisol response does not directly influence the effects of acute stress on source monitoring performance.

To the best of our knowledge, this is the first time that the effects of acute stress on source memory have been studied. Our findings are in support of theories contending that the role of the MTL in memory is markedly different from PFC contributions to memory (e.g., Moscovitch \& Winocur, 2002). Although acute stress and/or elevated GC levels tend to undermine declarative memory in a variety of ways (Het et al., 2005; Wolf, 2003), this study suggests that PFC regulated source monitoring performance benefits from stress. Perhaps, it is improved cerebral blood flow to the PFC during psychological stress (e.g., Wang et al., 2005) that accounts for the beneficial effect of stress on source monitoring performance. Alternatively, since source monitoring indices did not differ between high and low cortisol responders and these indices showed no significant correlation with delta cortisol increases, this seems to suggest that the noradrenergic system (e.g., via the release of noradrenaline) is implicated in the source memory enhancing effect of stress. Clearly, this issue needs further study. In any event, our findings seem to indicate that stress has differential effects on memory performance, depending on whether the memory test is regulated by the PFC or the MTL.

It should be acknowledged, though, that the current study's design cannot rule out that the source memory enhancing effect may be related to the MTL. That is, while neuropsychological (e.g., Janowsky et al., 1989; Schacter et al., 1984) and functional imaging studies (e.g., Dobbins et al., 2002, 2003; Mitchell et al., 2004; Raye et al., 2000; Slotnick et al., 2003) have demonstrated that source monitoring critically relies on the PFC, source monitoring is also related to MTL functioning. However, given that exposure to acute stress prior to retrieval has typically been associated with memory impairing effects (e.g., Domes, Heinrichs, Rimmele, 
Reichwald, \& Hautzinger, 2004; Kuhlmann, Piel, \& Wolf, 2005b), the present data thus seem to indicate that the source memory enhancing effect is PFC driven.

Because in the current study participants were exposed to the psychosocial stressor only after the SMA phase, our findings showing enhanced source monitoring performance cannot be attributed to differences in encoding between the two groups. Whether acute psychosocial stress given before or during the encoding phase can result in altered source monitoring performance therefore remains to be determined. Research on the effects of acute stress on MTL regulated declarative memory performance indicates that memory facilitation may occur when GC receptors are moderately stimulated while mineralocorticoid receptors are simultaneously totally saturated. When GC receptors are fully occupied, on the other hand, high GC levels may be disadvantageous to memory (e.g., de Kloet et al., 1999). At present, one can only speculate as to whether such differential effects can also be found for PFC regulated source monitoring tests. Also, it remains unclear whether higher doses (e.g., via GC administration) would results in enhanced source monitoring performance.

Some comments as to the limitations of this study are worthy of note. To begin with, the present study used a relatively homogenous (i.e., male undergraduate) sample of participants. Therefore, it remains to be seen whether its findings can be generalized to other populations (e.g., older adults, women). Secondly, the present study employed a source monitoring test that was specifically designed to elicit internal-internal source monitoring errors. Future work should investigate whether these findings also translate to other (i.e., external-external and internal-external) source monitoring tests. Finally, to directly compare the effects of acute stress on MTL and PFC regulated memory tasks, studies including independent memory tests of both types are needed.

In sum, the present study showed that exposure to acute psychosocial stress can result in improved internal-internal source monitoring performance. Although the current results seem to suggest that cortisol does not play a major role in these effects, the precise neurobiological mechanisms of these effects remain unclear. Future work using functional neuro-imaging techniques like, for example, Positron Emission Tomography (PET) or functional Magnetic Resonance Imaging (fMRI) could shed further light on this issue. 


\section{Delayed recall of childhood sexual abuse memories and the awakening rise and diurnal pattern of cortisol}

This chapter is an adapted version of: Smeets, T., Geraerts, E., Jelicic, M., \& Merckelbach, H. (in press). Delayed recall of childhood sexual abuse memories and the awakening rise and diurnal pattern of cortisol. Psychiatry Research. 


\section{Summary}

Traumatic stress associated with childhood sexual abuse (CSA) may results in chronic alterations of stress-sensitive neurochemical systems (e.g., the hypothalamic-pituitary-adrenal axis and sympathetic-adrenal medullary activity). Some authors have suggested that these alterations might help explain why some individuals, after a period of inability to remember, demonstrate delayed recall of CSA memories (i.e., "recovered" memories). The present study is the first study that explored morning cortisol responses and circadian cortisol profiles among women with recovered $(n=7)$, repressed $(n=8)$, or continuous $(n=6)$ memories of CSA and women without a history of CSA $(n=9)$. Although there were group differences in current depression and post-traumatic stress symptoms, we found no differences in cortisol awakening response or daytime profile between women reporting recovered, repressed, or continuous memories of CSA as compared to women without a history of CSA. Implications for neurobiological models intended to explain the delayed recall of CSA are discussed.

\section{Introduction}

Reports of individuals suddenly recovering previously inaccessible memories of past traumas, in particular experiences of childhood sexual abuse (CSA), have generated heated debate. Many clinicians (e.g., Scheflin \& Brown, 1996; Alpert et al., 1998) believe that the traumatic nature of CSA can render its victims amnesic for CSA episodes. They assume that in repressed and recovered memory cases special mechanisms termed repression or dissociation produce periods of psychogenic (i.e., dissociative) amnesia. Other authors, however, have questioned the accuracy of repressed and recovered memories. They argue that there is no sound evidence for special mechanisms linking trauma to dissociative amnesia (e.g., Pope, Hudson, Bodkin, \& Oliva, 1998; Roediger \& Bergman, 1998; McNally, 2003; Loftus, 2004; Kihlstrom, 2006).

Some authors (e.g., Howe, 1998; Bremner, 2001) have argued that biological factors rather than poorly defined mechanisms like repression underlie the loss and, perhaps, the recovery of traumatic memories. These factors would involve stress-sensitive neuroendocrine systems and their products, such as noradrenaline and cortisol. Authors stressing the importance of these factors reason that childhood abuse results in permanent effects on brain systems and circuits that mediate the stress response. Specifically, Bremner (e.g., 2003) maintains that early stress has lasting effects on the hypothalamic-pituitary-adrenal (HPA) axis. For example, an early stressor like maternal deprivation can result in increased glucocorticoid (GC) responses to subsequent stressors (Stanton, Gutierrez, \& Levine, 1988). Addition- 
ally, at least in nonhuman primates damaging early stressors can result in lasting effects on behaviours and elevated levels of corticotropin releasing factor in cerebrospinal fluid. Taken together, these and other reports suggest that "[...] early adverse experience permanently affects the HPA axis" (Bremner, 2003, p. 272).

Taking this line of reasoning one step further, Bremner (e.g., 2001) seems to postulate that altered cortisol levels following trauma exposure may account for the delayed recovery of CSA memories. There have indeed been reports of delayed recall of verbal material due to stress-induced cortisol elevations (e.g., Elzinga, Bakker, \& Bremner, 2005). Accordingly, long-term alterations in neuroendocrine systems such as the HPA axis may modulate the functioning of certain brain regions such as the hippocampus, the amygdala, and prefrontal cortex. Particularly sensitive to the effects of stress is the hippocampus, a brain area crucial to learning and memory. As early stressors (e.g., CSA) may permanently alter HPA axis functioning, this in turn might exert damaging effects on the hippocampus. By this argument, delayed recall of CSA is related to hippocampal dependent memory deficiencies that result from the long-lasting effects of childhood abuse on the HPA axis functioning.

If true, cortisol levels might be expected to differ in people who claim to have repressed or recovered CSA memories from people who have always remembered being abused, or for that matter from people without a history of CSA. Typically, activity of the HPA axis follows a circadian rhythm. In the morning, a rapid and acute increase in cortisol (i.e., the primary GC secreted by the adrenal cortex) levels can generally be observed, with a peak occurring at about 30 minutes after awakening (Pruessner et al., 1997; Wüst, Wolf, Hellhammer, Federenko, Schommer, \& Kirschbaum, 2000b). Thereafter, cortisol levels continually decrease during the remainder of the day. The awakening rise in cortisol is known to be affected by a host of factors including, for example, burnout (e.g., Pruessner, Hellhammer, \& Kirschbaum, 1999), vital exhaustion (e.g., Nicolson \& van Diest, 2000), chronic stress (e.g., Wüst et al., 2000b; Steptoe et al., 2003), and genetic predisposition (e.g., Wüst, Federenko, Hellhammer, \& Kirschbaum, 2000a). Moreover, larger morning responses are typically found during the week than in weekends, apparently reflecting higher stress on work days (e.g., Kunz-Ebrecht, Kirschbaum, Marmot, \& Steptoe, 2004).

So far, no study has investigated cortisol secretion in individuals reporting recovered, repressed, or continuous CSA memories, compared to people with no history of CSA. The aim of the present study was therefore to assess whether women with recovered, repressed, or continuous CSA memories or without a CSA history differ in two cortisol secretion parameters, namely their response to morning awakening and their diurnal pattern. One would predict differences in the circadian rhythm of cortisol secretion between these groups if massive bursts of GCs in response to CSA can, indeed, initiate neurochemical alterations that undermine memory (e.g., Bremner, Krystal, Charney, \& Southwick, 1996). More specifically, 
one would expect heightened morning and diurnal cortisol levels in recovered and repressed memory patients compared to those in continuous memory patients and non-traumatized controls. On the other hand, a recent study suggests that lowered rather than raised cortisol levels in response to awakening are linked to a total inaccessibility of memories (e.g., Wolf, Fujiwara, Luwinski, Kirschbaum, \& Markowitsch, 2005). Based on that study, one would expect that those individuals who claim to have repressed their CSA memories show deregulations in morning cortisol responses. It should be noted though that patients suffering from posttraumatic stress disorder (PTSD) are characterized by lower blunted responses to awakening and lower diurnal cortisol levels (e.g., Yehuda, 2002). Therefore, PTSD symptomatology should be taken into account when studying cortisol levels in women with a history of CSA, especially given reports of memory deficits in women with CSA related PTSD (e.g., Bremner, Vermetten, Afzal, \& Vythilingam, 2004).

\section{Methods}

\section{Participants}

Participants were recruited through advertisements in local newspapers as part of an ongoing project on CSA memories and psychopathology (e.g., Geraerts, 2006; Geraerts, Smeets, Jelicic, van Heerden, \& Merckelbach, 2005; Geraerts, Smeets, Jelicic, Merckelbach, \& van Heerden, 2006). After having given informed consent, all women underwent a semi-structured memory interview performed by E.G. On the basis of this interview, participants were classified as belonging to one of four groups. The first group consisted of women who said that after a period of having been unable to remember CSA episodes, they had subsequently recalled memories of CSA (i.e., recovered memory group). Women with this type of CSA memories had either recovered their memories during psychiatric treatment or after having been exposed to certain cues (e.g., a friend who told about CSA experiences, the birth of their own child). Women in the second group firmly believed they had been sexually abused as a child but had no memories of the abuse (i.e., the repressed memory group). They reported a wide range of symptoms that they thought were indicative of a CSA history (e.g., relationship problems, depressive symptoms, and eating disorders). Women who reported that they had always remembered being abused as a child were included in the third (i.e., continuous memory) group, while the fourth (i.e., control) group consisted of women who said they had no history of childhood or adulthood sexual abuse. As we were unable to verify whether or not participants had experienced CSA events, the terms "recovered", "repressed", "continuous", and "no CSA background" describe the belief that participants had about their own history. 
None of the participants in the recovered, repressed, or continuous group self-reportedly stated that they had experienced (potentially) traumatic events in their lives other than CSA. Participants in the control group all indicated that they were free of having experienced traumatic events in their lives. Only participants who had a normal body mass index (BMI) were included in this study.

Exclusion criteria included being (post-)menopausal and suffering from chronic fatigue or a current psychotic disorder. None of the participants met criteria for current or lifetime psychiatric disorders including, for example, major depressive disorder (MDD). Ten participants were excluded from analysis because they suffered from endocrine disorders (e.g., adrenal insufficiency, thyroid disease; $n=3$ ), serious medical diseases (e.g., fybromyalgia; $n=3$ ), or were on medications known to affect the HPA axis (e.g., antidepressants, synthetic corticosteroids; $n=4$ ). This resulted in a group of 30 women suitable for analyses including 7 recovered, 8 repressed, and 6 continuous memory patients, and 9 non-abused controls. All test protocols were approved by the standing ethical committee of the Faculty of Psychology, Maastricht University.

\section{Materials}

The Dissociative Experiences Scale (DES; Bernstein \& Putnam, 1986; Cronbach's $\alpha=.92)$ is a widely used self-report instrument containing 28 items describing dissociative phenomena, such as feelings of derealization, depersonalization, and disturbances in identity, memory, awareness, and cognition. Participants indicate on 100-mm Visual Analogue Scales (anchors: not at all and very much) the frequency with which they experience the phenomena described by each item. Scores on the individual items are averaged to obtain a mean DES score, with higher scores indicating a higher frequency of dissociative symptoms. The psychometric qualities of the DES are well established (e.g., Van IJzendoorn \& Schuengel, 1996).

The Beck Depression Inventory (BDI; Beck, Ward, Mendelson, Mock, \& Erbaugh, 1961; $\alpha=.81)$ is a widely-used and extensively validated self-report scale designed to evaluate depressive symptoms in clinical as well as in research settings (for a review, see Beck, Steer, \& Garbin, 1988). The BDI comprises 21 items scored on 4-point scales (anchors vary with items). Total BDI scores range from 0 to 63, with higher values representing more depressive symptoms.

The Posttraumatic Stress Symptom Scale-Self-Report Version (PSS-SR; Foa, Riggs, Dancu, \& Rothbaum, 1993; $\alpha=.92$ ) is a self-report measure comprising 17 items corresponding to the re-experiencing, avoidance, and arousal symptoms of PTSD. Participants rate the frequency of each item on 4-point scales ranging from 0 (not at all or only one time) to 3 (five or more times a week/ almost always). A total score can be obtained by summing across items. Psychometric evidence that supports the PSS-SR as a screening tool for PTSS can be found in Wohlfarth, van den Brink, Winkel, and ter Smitten (2003). 


\section{Procedure}

After having given informed consent, participants were contacted by mail and pro-

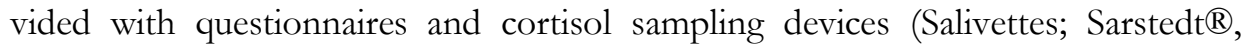
Nümbrecht, Germany), as well as detailed instructions on how and when to collect all data. Participants collected the saliva samples at home on two consecutive weekdays. Cortisol awakening response and diurnal pattern were assessed as follows. For the awakening rise, all participants obtained the first saliva sample immediately upon awakening, followed by additional samples collected 15 minutes, 30 minutes, and 45 minutes thereafter (for a rationale, see Pruessner et al., 1997). The diurnal pattern of cortisol secretion was characterized by saliva samples collected at $09.00 \mathrm{~h}, 11.00 \mathrm{~h}, 15.00 \mathrm{~h}$, and $20.00 \mathrm{~h}$. Participants were instructed to closely follow the guidelines for data collection and were asked to record time of awakening and times of saliva sampling. Participants were also asked to rate the quality of their night's sleep on a $100 \mathrm{~mm}$ VAS (anchors: $0=$ very bad; $100=$ very good; with 50 being the quality of a typical night's sleep). After collection of all saliva samples, participants returned the completed forms and sampling devices in a self-addressed envelope.

Upon return, uncentrifuged saliva samples were stored at $-40^{\circ}$ Celsius. Salivary free cortisol levels were determined in duplicate by direct radioimmunoassay (RIA; University of Liège, Belgium), including a competition reaction between 125iodohistamine-cortisol and anti-cortisol serum made against the 3-CMO-BSA conjugate. After overnight incubation at $4^{\circ}$ Celsius of $50 \mu \mathrm{l}$ of saliva, separation of free and antibody-bound 125iodohistamine-cortisol was performed via a conventional 'second antibody' method. This way, the lower detection limit of the assay was $0.1 \mathrm{nmol} / 1$. Mean intra- and inter-assay coefficients of variation were less than 4.3 percent and 9 percent, respectively. Cortisol levels for each sampling time were averaged for the two consecutive days. However, cortisol samples that according to self-report deviated more than 3 minutes from the standard time of measurement for the morning response or that deviated more than 15 minutes from the standard times for the daytime profile were discarded from further analysis. This was the case for 15 (3.1 percent) of all measurements. In these cases, cortisol data reflect only one data point instead of the average across two days.

\section{Statistical analyses}

Group differences in scores on the psychometric instruments were evaluated with univariate Analyses of Variance (ANOVA's) and follow-up $t$-tests. Cortisol data were examined for outliers, but none were identified. To normalize distributions, cortisol levels were subjected to natural log transformation before analysis. A 4 (group: recovered vs. repressed vs. continuous vs. controls) $\times 4$ (time: wake-up vs. $\mathrm{t}+15$ vs. $\mathrm{t}+30$ vs. $\mathrm{t}+45$ ) ANOVA was used to analyze the awakening rise, with time as the repeated measure factor. Similarly, diurnal patterns were analyzed using 
a 4 (group) $\times 4$ (time: $09.00 \mathrm{~h}$ vs. $11.00 \mathrm{~h}$ vs. $15.00 \mathrm{~h}$ vs. $20.00 \mathrm{~h}$ ) ANOVA. Additionally, univariate ANOVA's were used to test group differences in the cortisol Area Under the Curve (AUC; see Pruessner et al., 1997; Pruessner, Kirschbaum, Meinlschmid, \& Hellhammer, 2003) with respect to increase (AUCi) for morning responses. Likewise, the Awakening Cortisol Response (ACR) was computed as $[(t+30)-($ wake-up)] (see, for example, Clow, Thorn, Evans, \& Hucklebridge, 2004). Following Sephton, Sapolsky, Kraemer, and Spiegel (2000) and Weissbecker, Floyd, Dedert, Salmon, and Sephton (2006), we quantified the diurnal cortisol slope $(\beta)$ by estimating how each participant fitted the normal descending profile. To this end, a series of multiple regressions with cortisol values regressed upon the time of collection (i.e., cortisol sample) individually for each participant. Larger $\beta$ values represent flatter slopes and, thus, slower declines in cortisol. Steeper slopes (smaller $\beta \mathrm{s}$ ), on the other hand, indicate that cortisol decreases more rapidly. Finally, we computed Daily Average Cortisol (DAC; Gunnar, Morison, Chisholm, \& Schuder, 2001; Nicolson, 2004) by first standardizing cortisol measures over all participants at each sampling time and then averaging these Zscores for each participant. Pearson product-moment correlations (two-tailed) were used to examine whether questionnaire scores (DES, BDI, and PSS-SR) were related to cortisol awakening responses (AUCi and ACR) and diurnal cortisol values (DAC and $\beta \mathrm{s}$ ). Greenhouse-Geisser corrected $p$-values are reported when sphericity assumptions were violated. Partial Eta squared $\left(\eta_{p^{2}}{ }^{2}\right.$ is provided as a measure of effect size. Unless otherwise specified, alpha was set at .05 and adjusted (Bonferroni) for multiple comparisons where necessary.

\section{Results}

\section{Psychometric data}

Table 6.1 shows demographic and psychometric data of the four groups. As can be seen, groups did not differ with respect to age $\left[F(3,26)<1 ; p=.60 ; \eta_{p}{ }^{2}=.07\right]$. Pearson Chi-square Exact tests showed that groups were similar with respect to the proportion of women who smoked $\left[\chi^{2}(3, N=30)=1.04 ; p=.95\right.$; Cramer's $V$ $=.16]$, but that women in the repressed and the recovered group were more likely to have received psychiatric treatment related to their alleged CSA history than women in the continuous or control group $\left[\chi^{2}(3, N=30)=24.19 ; p<.001\right.$; Cramer's $V=.79]$. Groups also differed in their mean scores on the DES $[F(3,26)$ $\left.=10.79 ; p<.001 ; \eta_{p}{ }^{2}=.55\right]$ and the PSS-SR $\left[F(3,26)=3.82 ; p<.05 ; \eta_{p}{ }^{2}=.31\right]$. Follow-up $t$-tests showed that the participants reporting recovered, repressed or continuous CSA memories scored significantly higher on dissociative symptoms (DES) and post-traumatic stress disorder symptoms (PSS-SR) than the control group [all $t$ s $>2.29$; all $p$ 's $<.05$ ]. Overall, groups did not differ with respect to 
current symptoms of depression $(\mathrm{BDI})\left[F(3,26)=2.05 ; p=.13 ; \eta_{p}^{2}=.19\right]$, but a posteriori $t$-tests indicated that the repressed and continuous CSA groups were more depressed than the control participants [both $t^{\prime} \mathrm{s}>2.36$; both $p$ 's $<.05$ ].

Table 6.1. Demographic data, wake-up times, quality of sleep data, and mean $( \pm S D)$ scores of the four groups on measures of dissociation (DES), current depression (BDI), and PTSS symptoms (PSS-SR).

\begin{tabular}{|c|c|c|c|c|}
\hline & $\begin{array}{l}\text { Recovered } \\
\quad(n=7)\end{array}$ & $\begin{array}{l}\text { Repressed } \\
\qquad(n=8)\end{array}$ & $\begin{array}{c}\text { Continuous } \\
\qquad(n=6)\end{array}$ & $\begin{array}{c}\text { Control } \\
(n=9)\end{array}$ \\
\hline Age & $40.7(12.6)$ & $43.9(6.7)$ & $36.5(10.5)$ & $43.0(12.2)$ \\
\hline Ratio current smokers (yes/no) & $1 / 6$ & $2 / 6$ & $2 / 4$ & $2 / 7$ \\
\hline Ratio psychiatric treatment (yes/no)a & $7 / 0$ & $7 / 1$ & $2 / 4$ & $0 / 9$ \\
\hline Wake-up time & 07.14h & $07.17 \mathrm{~h}$ & $07.35 \mathrm{~h}$ & $07.10 \mathrm{~h}$ \\
\hline Quality of sleep & $58.9(15.9)$ & $55.0(24.0)$ & $50.4(20.9)$ & $69.9(21.6)$ \\
\hline DES & $22.2(11.1)$ & $34.4(13.8)$ & $23.1(4.2)$ & $8.7(3.6)$ \\
\hline BDI & $10.1(8.8)$ & $13.5(6.6)$ & $13.2(3.3)$ & $6.7(5.3)$ \\
\hline PSS-SRc & $14.6(10.8)$ & $20.3(13.4)$ & $14.8(3.2)$ & $5.6(4.3)$ \\
\hline
\end{tabular}

Notes: ${ }^{a}$ Repressed $>$ controls, recovered $>$ controls, and recovered $>$ continuous; $p<.05$; b Repressed $>$ controls, recovered $>$ controls, continuous $>$ controls; $p<.05 ;{ }^{c}$ Repressed $>$ controls; $p<$ .05

\section{Wake-up time and quality of sleep}

Table 6.1 shows wake-up times and quality of sleep data for the four groups. As can be seen, participants in the control group on average tended to wake up 4 minutes, 7 minutes, and 25 minutes earlier than women in the recovered, repressed, or continuous memory group, respectively. Data on subjective sleep quality were missing for one participant in the repressed memory group. Ratings $(M \pm$ $S D)$ of participants' sleep quality averaged over two consecutive days did not differ significantly between the 4 groups $\left[F(3,25)=1.23 ; p=.32 ; \eta_{p}^{2}=.13\right]$.

\section{Cortisol responses to awakening ${ }^{15}$}

Clear-cut morning cortisol responses were observed in all groups (see Figure 6.1). Accordingly, the repeated measures ANOVA showed a significant main effect of time $\left[F(3,78)=14.95 ; p<.001 ; \eta_{p}^{2}=.37\right]$, reflecting the expected morning rise in cortisol. However, the critical Time $\times$ Group interaction $[F(9,78)=1.20 ; p=.33$;

\footnotetext{
15 To control for possible effects of age, time of awakening, current symptoms of PTSD (PSS-SR), and current depression (BDI), the reported analyses pertaining to awakening responses and diurnal profiles were repeated with these factors included as covariates. However, no discrepancies were found between the results with these covariates included and the results reported here.
} 
$\left.\eta_{p}{ }^{2}=.12\right]$ and main effect of group $\left[F(3,26)=1.12 ; p=.36 ; \eta_{p}{ }^{2}=.11\right]$ fell short of significance. Likewise, analysis of the AUCi showed that groups did not differ in AUCi in response to awakening $\left[F(3,26)=0.85 ; p=.48 ; \eta_{p}{ }^{2}=.09\right]$, means being 16.66 ( \pm 7.05$), 10.98( \pm 12.18), 8.38( \pm 8.21)$, and $10.73( \pm 10.59)$ for the recovered, repressed, continuous, and control group, respectively. The ACR also did not differ between groups $\left[F(3,26)=1.18 ; p=.34 ; \eta_{p}{ }^{2}=.12\right]$.

\section{Morning response}

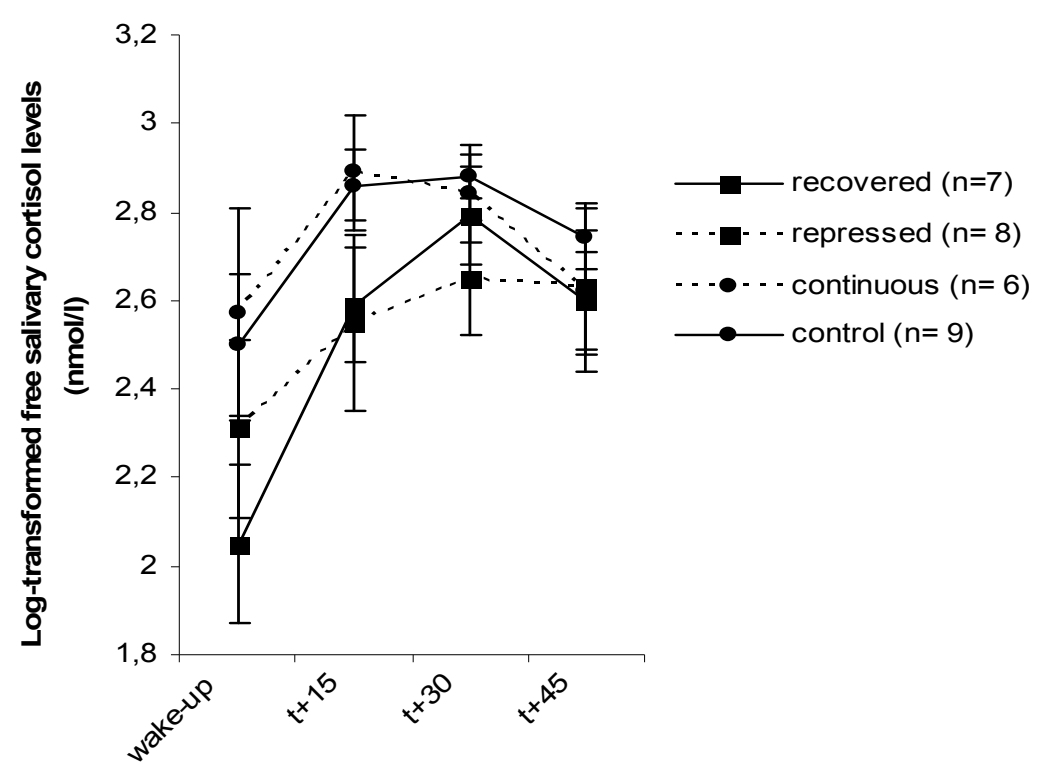

Time

Figure 6.1. Morning cortisol rise in participants reporting recovered, repressed, or continuous memories of CSA and control participants without a history of CSA. Individual data points represent average mean values $( \pm S E)$ of two sampling days.

\section{Diurnal pattern of cortisol}

Normal diurnal pattern of cortisol secretion was apparent for all 4 groups (see Figure 6.2). As expected, the ANOVA showed a main effect of time $F(3,78)=130.30$; $\left.p<.001 ; \eta_{p}{ }^{2}=.84\right]$, with cortisol levels continuously declining over the course of the day. Importantly, no main effect of group $\left[F(3,26)=0.39 ; p=.76 ; \eta_{p}{ }^{2}=.04\right]$ or a Time $\times$ Group interaction $\left[F(3,78)=0.90 ; p=.51 ; \eta_{p}{ }^{2}=.09\right]$ emerged. In line with this, an ANOVA showed no differences between groups in DAC scores $\left[F(3,26)=0.40 ; p=.76 ; \eta_{p}{ }^{2}=.04\right]$. Last of all, no group differences were found with respect to diurnal cortisol slopes $(\beta \mathrm{s})\left[F(3,26)=1.63 ; p=.21 ; \eta_{p}{ }^{2}=.16\right]$. 


\section{Diurnal pattern}

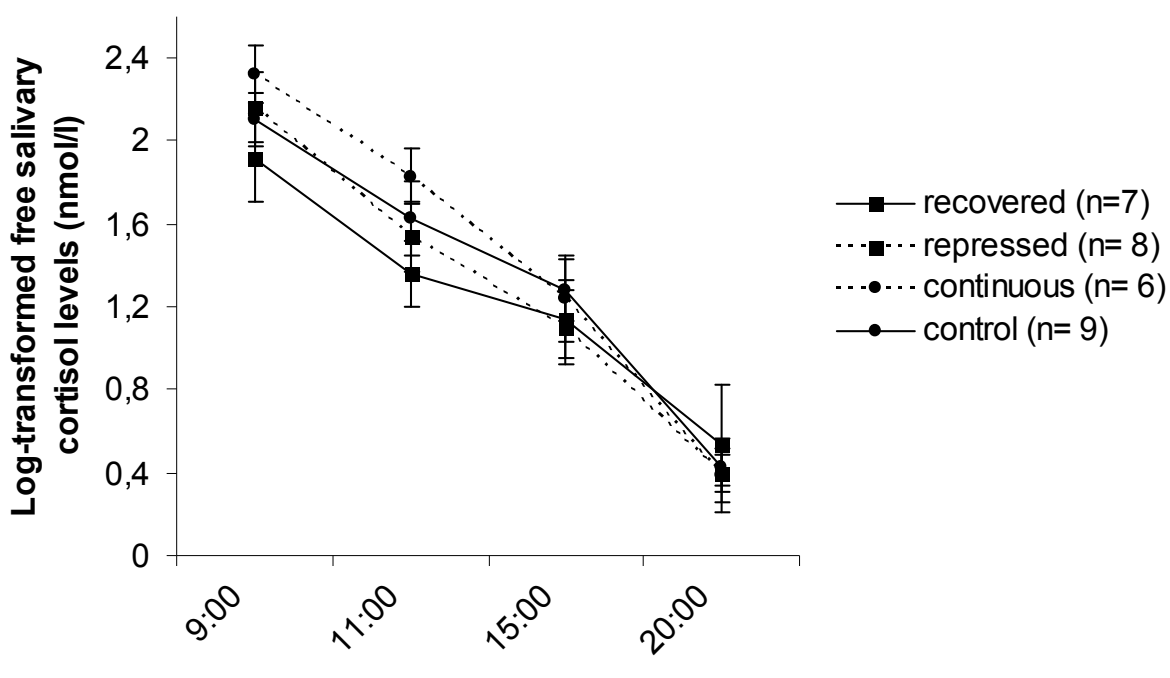

Time

Figure 6.2. Circadian rhythm of cortisol secretion for women with recovered, repressed, or continuous CSA memories and women without CSA histories. Data points represent average mean values $( \pm$ SE) of two sampling days.

Correlations between AUCi, ACR, DAC, $\beta$ 's and psychometric data

Correlations between DES, BDI, and PSS-SR scores, on the one hand, and morning cortisol response (i.e., AUCi and ACR) and daily cortisol secretion (i.e., DAC and $\beta$ s) parameters, on the other, all remained non-significant (all $r$ 's $<.22$; all $p$ 's $>$.23).

\section{Discussion}

Bremner (2001, p. 173) argued that "Modulation of memory function by cortisol and noradrenaline may represent a mechanism of delayed recall of childhood abuse. [...] Long-term dysregulation of these systems may result in chronic changes in the way memories are retrieved in abuse survivors with PTSD. For example, exaggerated cortisol release during stress in PTSD may result in an inhibition of memory retrieval. [...]. Both acute and chronic responses of these neurochemical systems to stress need to be considered in order to understand alterations in memory encoding and retrieval that we propose underlie delayed recall of child- 
hood abuse." This is the first study that compared cortisol levels in women with recovered, repressed, or continuous CSA memories to those of women without CSA history. The main results of the present study can be summarized as follows. First, contrary to what one would predict on the basis of theories popular in the recovered memory literature, we found no abnormalities in the cortisol patterns of women who had repressed, recovered, or continuous CSA memories. That is, neither the awakening rise nor the diurnal pattern in cortisol differed between CSA groups and controls, with all four groups showing a straightforward morning response and a typical diurnal rhythm of cortisol secretion. Second, morning responses in terms of AUCi and ACR and daily cortisol secretion (i.e., DAC and $\beta$ s) were not significantly related to measures of dissociation, current depression, or PTSD symptom severity.

Our psychometric data clearly indicate that the groups who reported CSA memories reported more trauma-related and dissociative symptoms than the control group. For example, on the PSS-SR the repressed memory group scored well above the cut-off point of 15 which, according to Wohlfarth et al. (2003), results in a sensitivity and specificity of .90 for predicting PSTD. Likewise, PSS-SR scores of the recovered and continuous memory groups came close to this cut-off point. Thus, there is little reason to assume that our CSA memory groups were free of psychiatric symptoms. Nevertheless, we found no association between posttraumatic or dissociative symptoms and cortisol levels. Our failure to obtain a correlation between dissociative symptoms and cortisol levels concurs with a study of Giesbrecht, Smeets, Jelicic, and Merckelbach (in press; see also Giesbrecht, 2006). These authors noted that cortisol responses following exposure to a psychosocial stressor were unrelated to measures of dissociation.

Although theories intended to explain delayed recall of CSA memories predict excessive cortisol levels in participants with repressed and recovered memories (e.g., Bremner, 2001), we found no differences in morning response or diurnal cycle of cortisol between women reporting recovered, repressed, or continuous memories of CSA, and women without a history of CSA. Thus, we found no support for the hypothesis that chronic alterations of neurochemical systems (e.g., cortisol) in reaction to trauma exposure can account for delayed recall of CSA memories. Additionally, the present data are at odds with Joseph's (1999) notion that fluctuations in daytime cortisol patterns are a possible hallmark of individuals with recovered memories of CSA.

All in all, our data provide no evidence for the idea that elevated cortisol secretion contributes to the temporary inaccessibility and subsequent recovery of traumatic memories. One could speculate, though, that our recovered memory participants had recovered their memories precisely because their cortisol levels had normalized. While such line of reasoning might make sense in the case of people with recovered CSA memories, it fails to account for the normal cortisol patterns in people reporting repressed CSA memories. Alternatively, there have been 
case studies suggesting that lowered rather than elevated cortisol levels are responsible for the inaccessibility of memories (e.g., Wolf et al., 2005). But then again, as far as the subsample of people claiming to have repressed their CSA memories is concerned, our data provide no support for this speculation.

Popular theories about the delayed recall of trauma memories also emphasize that chronically elevated cortisol levels due to trauma exposure may serve as a neurobiological pathway to PTSD. However, most studies in this domain (e.g., Yehuda, Southwick, Nussbaum, Wahby, Giller, Mason, 1990; Yehuda, Kahana, Binder Brynes, Southwick, Mason, \& Giller, 1995; Yehuda, Golier, \& Kaufman, 2005; for a comprehensive review, see Yehuda, 2002) have found lowered cortisol levels in PTSD patients. Thus, in line with the present study, research on HPA functioning in PTSD patients seems to implicate that neurobiological models stressing cortisol elevations are not all that compelling.

Our findings suggest that neuroendocrine differences prior to or after trauma exposure are not the sole or even the best candidate to explain the inability to remember the trauma that is said to be characteristic of recovered memories. An alternative explanation is that individuals reporting recovered CSA memories do not exhibit an inability to remember the CSA episode, but rather suffer from subtle cognitive distortions. For example, research has shown that women reporting repressed or recovered memories tend to overestimate prior forgetting (i.e., the "forgot-it-all-along" effect; see Merckelbach, Smeets, Geraerts, Jelicic, Bouwen, \& Smeets, 2006; Geraerts, Arnold, Lindsay, Merckelbach, Jelicic, \& Hauer, in press). Together with the current findings, these and other studies (for an excellent review, see McNally, 2003) suggest that cognitive distortions rather than neurochemical alterations following trauma exposure might account for the phenomenal experience of recovered memories.

Several limitations of the current study deserve some comment. One important limitation is that the stringent exclusion criteria (e.g., medication) resulted in a small sample size. Future studies should attempt to replicate these findings using larger sample sizes. Note, however, that the effect sizes seem to indicate that larger samples would yield a similar pattern of results. There is the theoretical possibility that sampling problems, such as non-compliance with the protocol, contributed to our non-significant findings. However, self-reported sampling times showed only small deviations from the planned sampling times. Furthermore, one could question whether self-reported quality of sleep and the resulting non-significant differences between groups on these ratings is sufficiently valid to rule out the possibility that sleep quality confounded the present results. In addition, though it is unlikely that menstrual cycle phase influenced the awakening cortisol responses (e.g., Kudielka \& Kirschbaum, 2003), our failure to control for menstrual cycle phase may have confounded the diurnal cortisol values. We also acknowledge that a prospective, within-subject study would be better to investigate HPA axis changes over time, but given the studied population one can see that such an endeavour would 
be next to impossible. Finally, while participants reported not to have suffered potentially traumatic experiences (other than CSA) in their lives, another measure of life stressors would have been desirable. Also, non-disclosure of other traumatic experiences cannot be ruled out.

In sum, our findings provide no evidence for the idea that cortisol levels are chronically altered in women with recovered, repressed, or continuous CSA memories. As things stand, neurobiological models stressing cortisol dynamics as an important contribution to recovered or repressed memories do not seem to be very promising. 


\section{Trying to recollect past events: Confidence, beliefs, and memories}

This chapter is an adapted version of: Smeets, T., Merckelbach, H., Horselenberg, R., \& Jelicic, M. (2005). Trying to recollect past events: Confidence, beliefs, and memories. Clinical Psychology Review, 25, 917-934. 


\section{Summary}

Numerous studies claim to have shown that false memories can be easily created in the laboratory. However, a critical analysis of the methods employed in these studies indicates that many of them do not address memory in the strict sense of the word. Instead, some of these studies assess the confidence that participants have in a fictitious (childhood) event, while others pertain to false beliefs about childhood events. While it is difficult to draw precise demarcation lines, we argue that inflated confidence, false beliefs, and false memories are different phenomena. Keeping the origins of these studies in mind (i.e., people who file lawsuits on the basis of their recovered memories), we propose that a fruitful, but stringent definition of false memories would incorporate their consequences. Thus, we argue that this research domain would profit from studies looking explicitly at whether experimental manipulations intended to implant false memories have overt behavioural consequences.

\section{Introduction}

Since the pioneering work of Bartlett (1932), research on memory has only gradually given up its preoccupation with accurate reproduction of previously learned words (Koriat, Goldsmith, \& Pansky, 2000). Today, however, there is a broad consensus among psychologists about the inherently constructive nature of episodic memory and its proneness to distortions. Two laboratory paradigms have contributed to this consensus (Dhodia \& Metcalfe, 1999; Mazzoni, 2002; Reyna, 2000). The first is the classic misleading information paradigm, in which participants are provided with misleading information about an event that they witnessed. When participants are later asked to recall the event, some of them will give false details on the basis of the misleading information. For example, in a study by Ackil and Zoragoza (1998), participants watched a movie fragment about boys at a summer camp showing one of the individuals tripping and falling. Next, the researchers suggested that that person was bleeding, although the video fragment had not shown this. When later asked where the individual was bleeding, some participants misremembered the suggested information as being accurate and gave detailed descriptions of where he was bleeding.

The second paradigm is the so-called false memory paradigm. In contrast to the misleading information paradigm, the false memory paradigm does not confront participants with misleading information. Here, false memories ${ }^{16}$ are said to

\footnotetext{
16 We define false autobiographical memories or pseudo-memories as recollections of events that never happened or that are recalled very differently from how they actually happened. In this chapter, we use the popular term false memory rather than the more neutral term pseudo-memory. We ac-
} 
result from participants' deductive inferences or internally created constructs that they subsequently mistake for externally experienced events (i.e., source monitoring errors, see Johnson, Hashtroudi, \& Lindsay, 1993; Lindsay, 1994). A good example of this approach is Bartlett's seminal study, where he had participants recount a story ("The War of the Ghosts"; see Bartlett, 1932) that was previously read to them. Due to their tendency to fill in memory gaps with internally generated schematic knowledge, participants changed or erroneously recalled certain details of the story (see for more recent examples, Bergman \& Roediger, 1999; Spiro, 1980).

Since the mid nineties both approaches have attracted considerable attention from psychologists as well as a broader audience. This interest in false memory research was mainly fostered by a cascade of cases in which people, during the course of a psychotherapeutic treatment, suddenly came to "recover" what seem to be previously inaccessible memories of traumatic childhood events (e.g., sexual abuse). There are conflicting opinions about the accuracy of these recovered memories. Many clinicians believe that such memories are essentially accurate (e.g., Andrews, Morton, Bekerian, Brewin, Davies, \& Mollon, 1995; Van der Kolk \& Fisler, 1995). Other authors, among whom are experimental as well as clinical psychologists, have pointed out that recovered memories may reflect an iatrogenic effect. They argue that certain psychotherapeutic techniques (e.g., hypnosis, dream interpretation) may very well elicit fantasies and imaginations that are experienced as autobiographical memories (e.g., Loftus \& Davis, 2006; Loftus \& Ketcham, 1994; Ofshe \& Watters, 1994). By this view, recovering memories during therapy may be nothing more than the construction of false memories. The credibility of recovered memories has lead to a heated controversy between both positions, with each side imposing high standards of proof upon the other side ${ }^{17}$ (see, for a more thorough analysis, Read, 1999).

While the recovered memory debate is still inspiring extensive reviews (e.g., Brewin, 2003; McNally, 2003), both positions seem to have gotten closer to one another (Ost, 2003; Schooler, 1999). For example, many authors now acknowledge that false memories recovered during therapy do exist (Health Council of the Netherlands, 2004; Sivers, Schooler, \& Freyd, 2002) and that accurate memories that have long not been thought about may be experienced as recovered memories. One positive side-effect of the recovered memory debate is that it has given rise to a huge experimental literature on how certain manipulations may affect people's judgments about their memories. This, in turn, has informed clinicians about the potential risks of certain therapeutic interventions (e.g., hypnosis, imagination exercises, dream interpretation). However, some critics (e.g., Freyd \& Gleaves, 1996)

knowledge, though, that false memory has negative connotations and that this has led to some terminological confusion.

${ }^{17}$ Lindsay $(1997$, p. 1) refers to this as the "entrenchment effect". 
have argued that generalizations from the experimental to the clinical context are difficult to make. Although points about generalization problems are often somewhat gratuitous, we believe that these critics might be right in one important respect. In what follows, we critically discuss methods, results, and conclusions that one commonly encounters in the experimental literature on false memories. This shows us that - despite the diversity in methodologies and results - most authors interpret their results in terms of false memories. In doing so, they often employ the terms false memory and pseudo-memory in a rather loose way. Consequently, these terms are at risk of becoming of little substance. Our goal is to illustrate this point. It is not our intention to provide the reader with an exhaustive review of the false memory literature. Rather, we want to advocate a more disciplined use of key concepts like confidence, false belief, and false memory. That many current research papers use these terms in a liberal way, does not mean that we regard them as unimportant or irrelevant. Instead, we argue that as long as studies in this area do not address the definitional issue, there are limits to their applicability to real life situations (e.g., the courtroom).

\section{Neurotransmitters as a metaphor for false memories}

The term neurotransmitter was first used by the Austrian researcher Otto Loewi in 1921. It started a revolution in biology as many physiologists promptly concentrated their research on the identification and functioning of neurotransmitters. This resulted in a rapid expansion of publications in this area of research. The proliferation of the term neurotransmitter led researchers to consider virtually all substances in the human brain as neurotransmitters. To avoid that it would become meaningless, redefining the term neurotransmitter using stringent criteria was inevitable (Axelrod, 1974). Thus, for example, a candidate substance only attains neurotransmitter status when it can be shown that it is involved in physiologic transmission (Boehning \& Snyder, 2003). Not surprisingly, then, a considerable amount of research on putative neurotransmitters focuses on their consequences: do they affect post-synaptic transmission?

In contemporary research, the term false memory is used in a rather liberal way. For example, some studies deal with a belief in a fictitious event (i.e., a false belief). In some cases, even this interpretation is too generous because a confidence estimate of a particular event rather than a belief is the dependent variable. Conferring a memory status upon beliefs and confidence estimates is like treating every substance in the brain as a neurotransmitter. Our point is not new. For example, Sivers et al. (2002, p. 182) noted that: "Another concern regarding false memory studies involves the degree to which the ideas that individuals generate are best described as false memories. Many studies that have been characterized as involving the creation of false memories have not actually caused individuals to 
specifically recall events that never occurred but rather have caused them to believe [italics added] that such events might have occurred." Our impression is that the experimental literature on false memories has largely ignored differences between confidence estimates, beliefs, and memories. To some extent this has to do with difficulties in defining the key concept of memory. Thus, for example, the neuropsychologist Dimond (1980) viewed memory as a pervasive feature of the brain ranging from immunological reactions to autobiography. ${ }^{18}$

\section{Imagination}

Studies intending to examine false memories often rely on techniques such as imagination inflation (Garry, Manning, Loftus, \& Sherman, 1996), dream interpretation (Mazzoni, Loftus, Seitz, \& Lynn, 1999a; Mazzoni, Lombardo, Malvagia, \& Loftus, 1999b), and personalized suggestions (e.g., Hyman \& Billings, 1998; Hyman \& Pentland, 1996; Loftus \& Mazzoni, 1998; Porter, Yuille, \& Lehman, 1999). There are also studies that draw on real life situations. A case in point is the study by Crombag, Wagenaar, and van Koppen (1996), who used the 1992 El-Al airplane crash on Amsterdam as their starting point (see below). Whatever the technique, authors claim to investigate false memories, either explicitly (e.g., Garry \& Polascheck, 2000; Mazzoni \& Memon, 2003; Paddock, Joseph, Chan, Terranova, Manning, \& Loftus, 1998) or by suggesting that the obtained results are pertinent to the recovered memory debate (e.g., Hyman \& Billings, 1998; Hyman \& Pentland, 1996; Mazzoni, Loftus, \& Kirsch, 2001).

Work on imagination inflation (e.g., Clancy, McNally, \& Schacter, 1999; Garry et al., 1996; Goff \& Roediger, 1998; Heaps \& Nash, 1999; Horselenberg, Merckelbach, Muris, Rassin, Sijsenaar, \& Spaan, 2000; Mazzoni \& Memon, 2003; Paddock et al., 1998; Paddock, Noel, Terranova, Eber, Manning, \& Loftus, 1999) offers examples of the terminological confusion that may arise. Here, participants are asked to fill out a Life Events Inventory (LEI; Garry et al., 1996), a questionnaire comprising 60 descriptions of certain events. Participants are asked to rate the probability that the events might have happened to them when they were a child. Sample items would be "Got in trouble for calling 911", "Broke a window with your hand", and "Won a stuffed animal at a carnival game". After one or two weeks, participants are asked to vividly imagine some of the situations that are described by the LEI items. Afterwards, participants are instructed to complete the LEI for a second time. Typically, researchers find that an event that was rated as

\footnotetext{
18 Over the past two decades, the concept of memory has extended its scope to include not only autobiographical recollections, but also perceptual priming, Pavlovian conditioned responses, and so forth. Thus, the broad use of the term false memory has its origin in the broadened concept of 'memory' itself (e.g., Tulving, 2000).
} 
unlikely increases in subjective probability after participants have imagined the event. Some authors argue that this imagination inflation effect tells us something about autobiographical memory distortions. For example, Paddock and colleagues (1998, p. S65) state that "[...] these results might provide insight into the mechanisms and processes by which false memories of childhood trauma may be created in psychotherapy." On a related note, Garry and Polascheck (2000, p. 6) opine that "A growing body of literature shows that imagining contrary-to-truth experiences can change memory [italics added]." Of course, these authors might be right. Yet, strictly speaking LEI items tap how confident participants are of having experienced a certain childhood event. They are not about having a detailed memory of that particular event.

Confidence is not the same as (false) memory. At most, inflated confidence constitutes a first step in the construction of false memories, just like L-DOPA is only a precursor of the neurotransmitter dopamine. Logically, it is perfectly possible to have inflated confidence without having false memories. More recent imagination inflation studies, like those by Mazzoni et al. (1999a, 1999b) or Mazzoni and Memon (2003) do not use the term false memory. Instead, these authors interpret their results in terms of false beliefs. In a review article, Loftus (2001, p. 585) pointed out that "Most of the studies of imagination inflation have shown shifts in belief, but have not explored whether actual memories or false memories accompany those shifts." However, given that imagination inflation studies rely on confidence estimates, even the term false belief may not always be justified. Suppose someone's confidence in a particular event (e.g., "Broke a window with your hand") increases from 1 to 3 on an 8-point scale (anchors: $1=$ definitely did not happen; $8=$ definitely did happen) as a result of an imagination exercise. Does this person really belief that this specific event happened to him or her? We do not think so. This is not to say that an increase from 1 to 3 on the 8-point confidence scale is trivial. In fact, it is highly relevant to, for example, clinical interventions (e.g., cognitive therapy; see Rachman, 2002; Radomsky, Rachman, \& Hammond, 2001) that intend to change the probability that clients attribute to certain events (e.g., not having a panic attack when entering a shop). But subjective probabilities are not the same as beliefs. In our opinion, it only makes sense to frame imagination inflation effects in terms of "belief" when confidence inflation is so large that it crosses the midpoint of the confidence scale. As far as we can see, only Heaps and Nash (1999) explicitly mention the differences between confidence, belief, and memory. Other authors summarize the results in this research domain in a way that does little justice to these differences. So, for example, Garry and Polascheck (2000; p. 8) say that "imagination can change autobiographies." Again, these authors might be right, but to date there is no imagination inflation study that fully documents this point. What can be said with some confidence, though, is that the effect of imagination not only inflates the confidence ratings of participants, but also undermines more fundamental memory processes. Thus, for example, Goff 
and Roediger (1998) found that imagining certain actions compromised the accuracy of source monitoring judgments (i.e., participants claimed to have performed rather than having imagined actions). Follow-up studies by Thomas and Loftus (2002) and Thomas, Bulevich, and Loftus (2003) indicate that these source monitoring errors persist even for actions that can be considered bizarre had they actually happened (e.g., kissing a magnifying glass).

\section{Suggestion}

Similar points can be raised about studies relying on dream interpretation or personalized suggestion. The first approach uses dream interpretation as the source of suggestion (e.g., Loftus \& Mazzoni, 1998; Mazzoni et al., 1999a, 1999b; Rassin, Merckelbach, \& Spaan, 2001; see also Pesant \& Zadra, 2004). Here, participants are asked to write down their recent dreams, on the basis of which the experimenter provides participants with suggestions about a childhood event that supposedly happened to them. Subsequently, the event is presented as the causal antecedent of their current dreams. Finally, participants have to rate their confidence in the suggested childhood event or have to provide memory reports on the event.

Another version of this approach uses suggestions embedded in hypnotic and non-hypnotic procedures to elicit certain information from participants (e.g., Spanos, Burgess, Burgess, Samuels, \& Blois, 1999; Spanos, Gwynn, Comer, Baltruweit, \& de Groh, 1989). Although these are well-designed studies that yielded highly interesting effects, they are sometimes very liberal in their terminology. For example, Mazzoni et al. (1999a) say that participants showing an increase in LEI scores subsequent to dream interpretation held beliefs (p. 141) about the suggested events. This might be a problematic conclusion (cf. supra). The authors go even further when they state that about half of their experimentally manipulated participants also had memories (p. 142) of these events. This conclusion is based on a single question that participants answered: "Would you describe to me the memory you have for this event? Try to remember as much as you can, but please try to spend only three minutes on each question" (Mazzoni et al., 1999a, p. 131). Next, two groups were formed: those who said to have memories of the events and those who did not. Participants were classified as having a memory of the event when "[...] subjects gave some indication [italics added] of having a memory" (Mazzoni et al., 1999a, p. 137). Participants were classified as having no memory for the event when they "[...] actually wrote 'no memory', 'cannot remember this really happening', or something similar [italics added]" (ibid.). Given this liberal way of defining memory reports, it remains to be seen whether the authors really succeeded in eliciting full-blown false memories.

In experiments relying on personalized suggestions (e.g., Hyman \& Billings, 1998; Hyman, Husband, \& Billings, 1995; Hyman \& Pentland, 1996; Loftus \& 
Mazzoni, 1998; Wade, Garry, Read, \& Lindsay, 2002), information about a fictitious event (e.g., spilling a punch bowl at a wedding) is suggested to participants by claiming that the information was gathered from participants' parents or close relatives. The frequently cited experiments of Hyman and Pentland (1996) and Hyman and Billings (1998) tried to elicit false memories by using this type of personalized suggestion (for a more recent example, see Ost, Foster, Costall, \& Bull, 2005). Participants were interviewed on several occasions about the suggested events and the researchers examined to what extent participants accepted the suggestions. Participants were eventually categorized in four groups. First, there was a "clear false memory" category containing participants, who said that they clearly remembered the particular event. These participants tried to complete their memories by providing progressively more details. Second, there was a "partial false memory" group of participants, who elaborated on the contextual details, but nevertheless said they had no specific memories of the incident. Thirdly, there was a group of participants, who tried to remember the incident, but said that they had no memories ("trying but no memory" group). Finally, there was a "no memory" group of participants, who essentially refused to attempt to recall information about the event. Using this classification format, Hyman and Pentland (1996) categorized 25 percent of their participants as "clear false memory" as opposed to 12.5 percent "partial false memory", 62.5 percent "trying but no memory", and 0 percent "no memory".

Though Hyman and Pentland's (1996) criteria were more precise than those of many other authors, the question arises to what extent participants in the first category (i.e., the clear false memory group) really remembered the suggested fictitious event. Participants in this category came up with all sorts of new, probably inaccurate details, indicating that they believed that the fictitious event truly took place. But whether they actually remembered the details they came up with or whether they merely proposed them as a way of speculating about the event remains unclear. ${ }^{19}$ Schwarz (1999) pointed out that participants usually adopt a cooperative attitude. Even when researchers pose non-sensical questions to participants, there is always a subgroup of participants who provides an answer as a way of being helpful to the researchers. Specifically, Schwarz (1999, p. 96) noted that "From a conversational point of view, the sheer fact that a question about some issue is asked presupposes that the issue exists." Thus, one wonders to what extent the "clear false memory" participants in Hyman and Pentland's (1996) study tried to be helpful by presenting speculations as memories to the researchers.

\footnotetext{
19 It is worthy of note that Hyman and Billings (1998) showed that false recollections obtained with their procedure were not related to participants' sensitivity to social demand. This is an important finding, but our point is, of course, broader. It may well be that most participants evaluated the experimental situation as a problem-oriented task and that some of them tried to be helpful by speculating about the fictitious event.
} 


\section{The El-Al crash}

Some authors have argued that suggestion may help create false memories for real life events. A study by Crombag, Wagenaar, and van Koppen (1996) used a public event (i.e., the $1992 \mathrm{El}-\mathrm{Al}$ airplane crash) to find out whether people would claim to have memories of non-existing television scenes of this event. Thus, Crombag and his colleagues sought to investigate their participants' memory for real television fragments of the El-Al Boeing 747 crashing into the buildings. To that end, the authors asked a simple forced choice (yes/no) question, namely "Did you see the television film of the moment the plane hit the apartment building?" (Crombag et al., 1996, p. 99). When answered affirmative, this was followed by one or more multiple choice questions, like "After the plane hit the building, there was a fire. How long did it take for the fire to start?" (ibid.). Crombag and colleagues found that over half of their participants $(55$ percent in study 1 and 66 percent in study 2) claimed to have seen the (non-existing) fragments. They speculated that the misleading suggestions embedded in their questions may have led their participants to come to believe that they saw a television fragment that in fact did not exist.

Because many participants gave detailed answers to the follow-up questions, Crombag et al. (1996, p. 102) also concluded that "[...] apparently these subjects had formed images [...]." In our view, the high percentage of participants claiming to have seen the film fragment does not necessarily mean that all of them actually had false memories or images. Another possibility is that participants, due to the highly suggestive context, drew upon general knowledge heuristics (e.g., "a plane crash causes an immediate explosion") rather than false memories when they answered the follow-up questions. As well, participants may have been eager to please the researchers and, hence, might have provided socially desirable answers without really believing them. In both cases, there is no need to postulate underlying false memories. ${ }^{20}$ In a highly similar study by Ost, Vrij, Costall and Bull (2002b), 44 percent of the participants were willing to report that they had seen television fragments of the 1997 fatal crash of Diana, Princess of Wales, when in fact, no such film material exists. Interestingly, these authors found evidence that compliance (i.e., eagerness to please) may be a key factor in explaining why so many of their participants claimed to have seen the non-existing fragment. Ost et al. do not claim that their participants had developed false memories. In interpreting their findings, the authors are conservative in that they state that participants "claimed to have seen" (e.g., p. 132) the television fragment of Princess Diana's car crash.

\footnotetext{
${ }^{20}$ Davis, Loftus, and Follette (2001, p. 151) noted that the study by Crombag and colleagues reflected false beliefs rather than false memories. They stated that "Clearly, these memories were nothing more than beliefs based on descriptions heard on T.V."
} 


\section{The story so far and its significance}

We may conclude, then, that experimental research on false memories is often vague and confusing when it comes to the point of whether effects exceed the level of weak or even strong beliefs. Of course, experimental work that shows how people can come to believe in fictitious events is fascinating and highly relevant. After all, there are patients who come to believe during therapy that they were the victim of childhood sexual abuse (CSA) without ever having memories of these events (McNally, 2003; Ost, Costall, \& Bull, 2001, 2002a). Indeed, these patients have much in common with patients who during therapy recover full-blown memories of being molested as children (McNally, 2003). For example, both groups are vulnerable to false alarms on recognition tasks (Clancy, Schacter, McNally, \& Pitman, 2000) and, contrary to what many clinicians believe, are not particularly skilled in forgetting trauma-related words (McNally, Clancy, \& Schacter, 2001). Still, it is wise to differentiate between belief and memory. Germane to this issue is that McNally, Clancy, Schacter, and Pitman (2000) also noted subtle differences between patients with recovered memories of abuse and patients with beliefs about childhood abuse. For example, McNally et al. (2000) reported that patients who merely believed in that they had been abused scored higher on measures of absorption and dissociation compared to patients with recovered memories of abuse, who in turn scored higher than patients who had always remembered their abuse.

Of course, false memories do exist. It would be foolish to deny that vivid memories of alien abduction episodes, held by some people who underwent hypnotic therapy, may be classified as false memories (Clancy, 2005; Clancy, McNally, Schacter, Lenzenweger, \& Pitman, 2002). A similar line of reasoning applies to the detailed descriptions some people give of their memories of previous life experiences (Gomperts, 1996; Horselenberg \& Merckelbach, submitted for publication). But, sometimes there is a clear asymmetry between belief and memory. For example, in his work on "retractors" (i.e., people who retract their allegations of CSA), Ost (2003, p. 134) noted that with many of these individuals memory is simply not the issue: "One retractor reported that she believed for 11 years that she had been abused, yet never actually came to remember the abuse." Ost goes on to add: "When someone becomes convinced that a past event occurred whilst simultaneously claiming not to be able to remember that event, then factors other than 'a memory' were clearly crucial in her decision."

More parametric research focusing on the phenomenal experiences of people claiming alien abductions, previous lives or CSA is required, because it could give us better clues as to how to draw the demarcation lines between confidence, belief, and memory. 


\section{Confidence estimates, false beliefs, and false memories}

Gardiner and Java (1993, p. 163) wrote that "The science of memory continues to be hampered by terminological confusion and excess. The same terms are often used to mean different things. Different terms are often used to mean the same things. Conceptual and theoretical progress would be easier if the use of terminology were to be reformed." We believe that their words bear relevance to false memory research. That is, this research domain would profit from stricter criteria for what counts as a false memory. A first step would be to make a distinction between confidence estimates about fictitious events, beliefs about such events, and false memories. Sporadically, authors do make this distinction..$^{21}$ Johnson and Raye (2000, p. 36), for example, noted that "People tend to use the word 'memory' when a mental experience or report of a mental experience is detailed, including information indicating that one experienced the event oneself, and they tend to use the word 'belief' when it does not have contextual details and for a broad range of mental experiences or reports that seem to assert present or past general states of affairs which may or may not involve personally experienced events (including the events from which the belief was derived, such as reading a newspaper)." Likewise, Read and Lindsay (1994, p. 429) stated that "[...] some cases of inaccurate delayed accusations might be better characterized as involving false beliefs rather than illusory memories." In a similar vein, Ost (2003, p. 135) noted that "The problems arising from false claims and also incorrectly rejected claims of sexual abuse are not just about 'memory' as traditionally conceived and, in some cases, may have very little to do with 'memories' at all, whether 'false' or 'recovered'." Differentiating between belief and memory is also important to experimental investigators, as it may be much easier to elicit false beliefs than false memories. Tulving (1985) argued that it is possible to distinguish between belief and memory on the basis of phenomenological experiences accompanying the retrieval of information. Thus, Tulving's remember-know distinction implies that if retrieval is accompanied by conscious recollection, participants experience a remember response. If, on the other hand, retrieval is accompanied by feelings of familiarity in the absence of conscious recollection, one is to speak of a know response (Gardiner \& Java, 1993; Tulving, 1985). Interestingly, some studies on false memories have collected direct remember-know judgments from their participants. For example, such data have been obtained in the context of the Deese-RoedigerMcDermott paradigm (DRM; Deese, 1959; Roediger \& McDermott, 1995). Although this approach might be informative as it strikes the heart of the point we want to make, some authors have noted that participants not always find it easy to

${ }^{21}$ Excellent chapters on the relationship between belief and memory of an event can be found in Schacter and Scarry (2000). On a minor point, also notice that Kopelman (1999) distinguishes between belief (delusions) and memory (delusional memory) among psychiatric patients. 
distinguish between remember and know judgments (e.g., Gardiner, Ramponi, \& Richardson Klavehn, 2002).

Several factors may modulate the confidence ratings that people give to particular childhood events. Among other factors, generic knowledge about the type of event (i.e., schematic knowledge), base rate of the event, fantasy proneness and suggestibility of the individual, and a lack of confidence in one's own memory ${ }^{22}$ may affect subjective likelihood estimates (e.g., Heaps \& Nash, 1999, Thomas et al., 2003). Whether one comes to believe the thought of having experienced a particular event depends to some extent on how plausible one judges the event to be (Hyman \& Kleinknecht, 1999; Loftus, 2003). If the prima facie plausibility is considered to be low, this will result in disbelief (denial of the thought). If, on the other hand, the subjective likelihood is judged to be high, one will often start to believe in and become convinced of actually having experienced the event. Germane to this issue is Pezdek, Finger, and Hodge's (1997) finding that highly plausible events (e.g., getting lost in a shopping mall) were more likely to be endorsed by their participants than less plausible events (e.g., having a rectal enema). Note that research also suggests that familiarity and plausibility are not static qualities. Thus, work by Goff and Roediger (1998) and Thomas and Loftus (2002) indicates that with multiple acts of imagination, people can be led to believe that they performed an unusual action (e.g., sit on a dice).

\section{Models}

Over the past 10 years or so, several models have been invoked to account for the development of false beliefs and false memories (e.g., Hyman \& Kleinknecht, 1999; Mazzoni \& Kirsch, 2002; Tousignant, Hall, \& Loftus, 1986). One of the first attempts was that by Tousignant et al. (1986; see also Schooler \& Loftus, 1986) and involved a principle known as Discrepancy Detection. According to this principle, "Recollections are most likely to change if a person does not immediately detect discrepancies between post-event suggestions and memory for the original event" (Schooler \& Loftus, 1986, p. 107-108). Discrepancy detection is assumed to be influenced by two factors: (1) the strength of the information that was originally encoded, and (2) the manner in which the post-event suggestion was presented to the participant. ${ }^{23}$ The implication of this is that (partial) amnesia is a necessary condition for the development of a full-blown false autobiographical memory.

\footnotetext{
22 This phenomenon, in which people come to doubt the quality of their own memory, is known as the memory distrust syndrome (Gudjonsson \& MacKeith, 1982).

23 A more recent version of the discrepancy detection principle can be found in Lindsay (1990), who in this context wrote about the discrimination hypothesis.
} 
More recently, Hyman and Kleinknecht (1999; see also Hyman \& Loftus, 1998) argued that the development of false (childhood) memories involves three processes: event acceptance (plausibility assessment), memory construction, and a source monitoring error. First, a person has to judge the suggested event as plausible. That is, the person should believe that the event could have happened. Factors that may have an effect on the plausibility assessment are the source of the suggestion, the nature of the event itself, and the likelihood of having personally experienced a similar event. Second, apart from believing that the suggested event is likely to have happened, a person "[...] must still construct a memory — an image with a narrative" (Hyman \& Kleinknecht, 1999, p. 180). Finally, one must misinterpret the constructed memory as an autobiographical memory. Thus, one has to make a source monitoring error and mistakenly claim the memory for an authentic personal memory. According to Hyman and Kleinknecht (1999, p. 181), "[...] all three processes are necessary for false memory creation and [that they] are somewhat independent in the sense that different factors influence each process."

A somewhat different conceptualization of false memories has recently been proposed by Mazzoni and Kirsch (2002). Although these authors noted that their model is in many ways consistent with that of Hyman and Kleinknecht (1999), they also point at one major discrepancy between both models. Thus, Mazzoni and Kirsch's (2002) model distinguishes between belief and memory. More specifically, these authors emphasize that autobiographical beliefs involve judgments about specific events, which guide the search for memories (i.e., recollective experiences).

With these models in mind, one could describe the various key concepts in this research domain as follows. Subjective probability (likelihood) of an event refers to a subjective estimate of the probability that the event reflects a genuinely experienced event. In line with Koehler (1991), one's overt expression of the subjective probability of a particular event is what we term confidence. A belief, on the other hand, emerges when one's confidence in the reality of an event is strong enough as to have little doubt about the veracity of the event. In that case, one comes to believe that one has experienced a certain event. This is primarily derived from other knowledge. Of course, beliefs may vary in strength and they may be true or false. When one beliefs in the veracity of a particular event and one has a concrete, narrative, episodic recollection together with an image of the event, one can speak of a memory of the event. Again, a memory can be true or false.

\section{Behavioural sequence}

Some authors fail to articulate the distinction between false belief and false memory. For example, Lampinen, Neuschatz, and Payne (1998, p. 182) define false memories as "[...] false beliefs about the past that are experienced as memories." Yet, in our opinion, false memories are more than just plain beliefs. That is, if peo- 
ple remember something happening, they will be likely to believe that it happened. However, believing in one's birth is not the same as having a memory of it (Mazzoni \& Kirsch, 2002). Moreover, not every belief or memory is of equal significance. A particular belief or memory is of demonstrable significance only when a person acts on it. Following the pragmatic tradition that considers memory in the service of overt behaviour (e.g., Fiske, 1992; Neisser, 1996), one could take the stance that the significance of a belief or memory can be derived from its behavioural sequence. This sequence can vary from passing the false information on to others (e.g., a confederate of the experiment), searching for additional information by talking to family or friends, or, in the case of a false confession, accepting a conviction. The recovered-false memory debate was, of course, fuelled by high publicity cases in which patients took legal action on the basis of their recovered memories. This leads us to conclude that the strongest demonstration of an experimentally induced false memory would be one in which participants not only come up with detailed reports about the false event, but also act on the basis of these reports. We are not alone in this conclusion. Thus, having discussed a new experimental approach in which imagination inflation was found to contribute to people's food aversion, Loftus (2003, p. 870) concluded that "One might then be able to show that false memories have consequences, that they do matter."

Recent work by Scoboria, Mazzoni, Kirsch, and Relyea (2004; see also Scoboria, Mazzoni, Kirsch, \& Jimenez, in press) suggests that confidence, false beliefs, and false memories can best be viewed as nested constructs. That is, having a memory of an event generally implies a belief that the event happened and, likewise, having autobiographical belief generally implies that the event is seen as plausible. However, if someone rates the probability of a certain event low or, for that matter, beliefs in the event, but shows no memory of it, this is not necessarily an end stage. When elaborating about a fictitious event, for example during therapy sessions in which imagination or hypnosis is repeatedly used, one's confidence in the event might increase. With more sessions, one may then shift from disbelief to belief that the false event has happened and eventually, one might shift from believing to actually constructing a false memory about a fictitious event. In current research on imagination inflation, several studies have found evidence that familiarity rather than reality monitoring failures drives the imagination inflation effect (e.g., Thomas et al., 2003). Perhaps, then, familiarity with the false event precedes increases in confidence and upward shifts in belief, while reality monitoring failures precede full-blown false memories. Moreover, response criterion ${ }^{24}$ shifts may be responsible for changing from disbelief to belief. An example might clarify this. Suppose that during the course of a treatment session, the therapist exposes his/her patient to the suggestion that childhood trauma is the source of the pa-

24 The consideration of how accurate information has to be judged in order for it to be reported. Mazzoni and Kirsch (2002) call this the pre-set criterion probability. 
tient's current complaints. Possibly, the patient will put his or her trust in the therapist. As a result, the patient may come to believe that he or she was a victim of CSA. At that point, however, the patient need not have any memories of the abuse. As time passes and after elaborating on the issue of abuse (e.g., with regression therapy or imagination exercises), clear, vivid, and detailed images of the abuse may emerge. However, only when the patient acts upon the basis of these images, like seeking confirmation from possible witnesses or confronting the alleged perpetrator, it becomes apparent that the patient takes his/her recovered images as real memories.

\section{Anxiety research}

Of course, there is no need for behavioural consequences to be exclusively related to memories. That is, one may act on the mere basis of a firmly held belief that does not possess any mnemonic qualities. Evidence for this comes from anxiety research on a cognitive bias termed Thought-Action Fusion (TAF; e.g., Rachman, 1993; Rachman \& Shafran, 1999) and from experimental work on indirect pathways to fear acquisition (e.g., Field, Argyris, \& Knowles, 2001; Field \& Lawson, 2003). Rachman and co-workers (e.g., Rachman, 1993; Rachman \& Shafran, 1999; Shafran, Thordarson, \& Rachman, 1996) have identified a cognitive distortion that is apparent in patients with Obsessive Compulsive Disorder (OCD). This cognitive distortion (TAF) refers to a bias that may increase people's sense of responsibility for their intrusive thoughts and, hence, can be regarded as a vulnerability factor for the development of clinical obsessions (e.g., Rachman \& Shafran, 1999; Rassin, Merckelbach, Muris, \& Spaan, 1999). TAF involves two separate components: probability and moral TAF. Probability TAF refers to OCD patients' tendency to believe that their intrusive thoughts increase the probability that a specific negative event will occur. Thus, OCD patients assume that having intrusive thoughts about, say, their parents suffering from an illness, will increase the probability that this will happen. Moral TAF refers to the belief that experiencing an intrusive thought (e.g., about an aggressive act) is morally equivalent to actually carrying out the prohibited action. There is ample evidence that OCD patients give high probability ratings for negative events as a result of their negative thoughts (e.g., Amir, Freshman, Ramsey, Neary, \& Brigidi, 2001; Shafran et al., 1996). Thus, these patients believe they can be held responsible for the anticipated negative event and this motivates them to engage in compulsive checking behaviour (e.g., Rachman, 2002). Clearly, these checking behaviours are based on the patients' sheer beliefs that they are responsible for future events.

Interestingly, Ladouceur and his colleagues (1995; experiment 2) demonstrated that beliefs about responsibility affect checking behaviours. Thus, Ladouceur and co-workers had their participants sort 200 pills (medication for a deadly 
virus) of 10 different colour combinations (20 capsules of each colour) that were initially placed in a bowl. Participants were instructed to put the pills in semitransparent bottles, and were either told that this was merely a pilot test (low responsibility group) or that their test performance would have a great and immediate impact (e.g., on the distribution of pills among poor people for which a wrong combination of colours could have lethal consequences). Compared to low responsibility participants, the high responsibility group exhibited a stronger preoccupation with errors and they experienced more subjective anxiety to make errors. More importantly, participants in the high responsibility group showed stronger hesitations in sorting the pills and they more often engaged in checking behaviours.

Another good example of behaviour associated with pure belief (i.e., without memory) can be found in the experimental work of Field and colleagues (e.g., Field et al., 2001; Field \& Lawson, 2003). In two experiments, Field et al. (2001) provided children aged 7 through 9 ( $N=40$ and $N=45$, respectively) with either positive (e.g., "very friendly") or negative (e.g., "very dangerous") information about toy monsters that were completely new to the children. Field et al. found that children showed increased scores on a fear-belief questionnaire concerning the monster about which they had received the negative information. In a subsequent study using Australian marsupials (i.e., the quoll, quokka, and cuscus) that were unfamiliar to the participants, Field and Lawson (2003) presented 6 to 9 year olds $(N=59)$ with negative, positive or neutral information about the animals. Not only did negative information significantly increase children's fear beliefs (measured by selfreport and implicit measures of the belief), but it also elicited avoidance behaviour. That is, children were more reluctant to approach a box which they believed contained the animal they had previously received negative information about. Evidently, increased fear beliefs were the antecedent of this behavioural consequence.

\section{A look at future research}

Experiments in the domain of anxiety research offer inspiring examples of how one can address the behavioural consequences of beliefs and memories. This is important because only a handful of studies concerned with false memories looked at the potential consequences of false recollections. An example may help to explain why it is informative to look at behavioural consequences. Suppose an individual has been ripped of for hundreds of dollars in purchasing a new car. When buying another vehicle, he/she will probably not go to the same car seller again. This, of course, has to do with remembering the bad investment. If, on the other hand, the individual sees it only as a remote possibility that the car seller has sold bad cars, he/she may very well visit the car seller for a second time. So, the behav- 
iour of the individual tells us something about his/her beliefs or memories about the car seller.

Consider this hypothetical experiment. Suppose that participants would imagine having experienced a traumatic childhood incident with a spider. Would they react with phobic anxiety when exposed to a spider? Or suppose one would tell participants that their dreams mean that as a child, they became nauseous each time they ate cereals for breakfast. Would that lead to these participants start to avoid eating cereals? As a matter of fact, Loftus (2003) recently found some tentative evidence that these consequences can, indeed, be elicited with typical false memory manipulations. When Alan Alda ${ }^{25}$ visited Loftus for television recordings, he completed a number of questionnaires about his food preferences, eating habits, and his personality (Loftus, 2003). During a follow-up session, Alda was told that an analysis of the questionnaires had revealed that he had an aversion to hard boiled eggs. Loftus and her research associates tried to convince Alda that as a child, he must have gotten sick after eating too many hard-boiled eggs. They succeeded, because after a while, Alda increasingly showed behavioural signs of believing in the authenticity of the fabricated story. During a picnic, Alda was offered a few hard-boiled eggs amongst other foods. Alda refused to eat the eggs. Of course, Alda's reluctance to eat the hard-boiled eggs may be due to a number of causes, but the example demonstrates how one might examine the behavioural consequences of false beliefs and false memories.

One research line that has explicitly focused on behavioural consequences of false beliefs and false memories is that concerned with false confessions. Kassin and Kiechel's (1996) classic study showed that it is relatively easy to obtain false confessions of healthy undergraduates. These authors instructed their participants to copy letters that were presented on a computer screen. Participants were told not to touch the Alt-key because otherwise the computer would crash. During the task, the computer did crash and participants were falsely accused of having touched the forbidden key. Next, participants were asked to sign a written confession and they were approached by a confederate of the experiment who acted as a naive subject. This confederate asked the participant about what happened. Kassin and Kiechel found that many participants (depending on the precise conditions between 35 percent and 100 percent) were wiling to sign a written confession. A smaller portion of them (depending on the conditions between 0 percent and 65 percent) related to the confederate how they had touched the forbidden Alt-key. At the very least, this suggests that these participants firmly believed that they had touched the forbidden key. That is, they had internalized the false confession.

A replication study by Horselenberg, Merckelbach, and Josephs (2003) explored whether false confessions would manifest themselves in more far-reaching

\footnotetext{
25 Alan Alda is the host of Scientific American Frontiers, a popular American t.v. show, and is widely known from his role as Hawkeye Pierce in the classic t.v. series $M^{*} A^{*} S^{*} H$.
} 
behavioural consequences. Following a procedure that was similar to that of Kassin and Kiechel, these authors investigated whether their falsely accused participants would accept a negative consequence of confessing, in this case paying a considerable amount of money. Horselenberg et al.'s results indicate that especially those participants who showed signs of internalization (42 percent of all participants), were willing to pay. A follow-up study by Horselenberg and associates (Horselenberg, Merckelbach, Smeets, Franssens, Peters, \& Zeles, 2006) shows that even when event plausibility is low and stakes are high (e.g., being falsely accused of exam fraud), some undergraduates falsely confess. Redlich and Goodman (2003) recently investigated whether children aged 12 and $13(n=32)$, children aged 15 and $16(n=32)$, and young adults aged 18-26 $(n=32)$ were willing to accept a highly aversive consequence of falsely confessing, namely returning for approximately 10 hours and re-enter the lost data. These authors found that, on average, 69 percent of their participants falsely confessed to hitting the forbidden key and, thus, were willing to accept the negative consequence. In our view, this type of approach offers good examples of how one may study the behavioural manifestations of firmly held misinterpretations (false beliefs) and false memories. ${ }^{26}$

Paying systematic attention to the behavioural consequences of false beliefs and false memories will enable us to formulate more fine graded models on how confidence may develop into beliefs and memories. Apart from theoretical issues, there is one practical reason for focusing on overt behavioural consequences of memory manipulations. As already noted, one key issue in recovered memories is that people who have them are so convinced of their accuracy that they act on the basis of their memories (e.g., by filing a lawsuit or by confronting family members). The parallel between laboratory experiments and real life examples of recovered memories will become much more compelling if these experiments involve overt behaviour (e.g., avoiding cues, signing a confession, talking to a confederate) that only makes sense when it is assumed that the actor holds firm beliefs or has detailed memories that he/she believes to be accurate.

\footnotetext{
26 An interesting parallel between false confessions and false memories can also be found in Ost et al. (2001). For a rationale on how false confessors can come to believe they are guilty and subsequently create false memories supporting those beliefs, see Henkel and Coffman (2004).
} 


\section{"Of course I remember seeing that film!"- How ambiguous questions generate crashing memories}

This chapter is an adapted version of: Smeets, T., Jelicic, M., Peters, M. J. V., Candel, I., Horselenberg, R., \& Merckelbach, H. (2006). "Of course I remember seeing that film!"'- How ambiguous questions generate crashing memories. Applied Cognitive Psychology, 20, 779-789. 


\section{Summary}

Although previous research has shown that people are willing to report having seen non-existent footages of high publicity events, no study has looked at the potential boundaries of what has been dubbed the crashing memory paradigm. We examined whether the ambiguity of interview questions may lead some people to affirm without much conviction having seen non-existent footages. Using ambiguous, specific high-suggestive, specific low-suggestive, or neutral questions, the current study asked 120 individuals whether they had seen non-existent footage of the assassination of the famous Dutch politician Pim Fortuyn. Replicating previous research, 63 percent of our participants in the ambiguous group falsely reported having seen the footage. This percentage dropped to 30 percent for the specific high-suggestive as well as the specific low-suggestive group, while still 27 percent of the neutral group were willing to make false reports. Our results demonstrate that crashing memories do depend on the way in which people are interviewed, but that question type does not fully account for these false reports.

\section{Introduction}

Eyewitness testimony is among the most potent types of evidence used in criminal and civil lawsuits. By and large, eyewitness testimonies hinge upon memories of events or facts that are in some way related to the legal issue at hand. Disconcerting is the fact that these memories are prone to various types of error, which under some circumstances may lead to inaccurate or even utterly wrong testimonies. That such errors may have far-reaching consequences is evidenced by the fact that mistaken eyewitness identifications account for over 80 percent of wrongful convictions in the United States (Scheck, Neufeld, \& Dwyer, 2001). The malleability of memory is not restricted to personally experienced events, but also applies to "memory" for highly exposed public events. That is, people may come to report having witnessed events that they, in fact, could not possibly have experienced. In their pioneering study, Crombag, Wagenaar, and van Koppen (1996) examined to what extent people would claim having seen television footage of the $1992 \mathrm{El}-\mathrm{Al}$ Boeing 747 crashing into apartment buildings in Amsterdam. To this end, participants were asked a straightforward yes/no question, namely "Did you see the television film of the moment the plane hit the apartment building?" (Crombag et al., 1996, p. 99). Crombag and colleagues found that over half of their participants (55 percent in study 1 and 66 percent in study 2) claimed to have seen the footage. This is remarkable given that there is no actual footage of the moment the El-Al airplane crashed into the buildings, although it is true that the event was extensively covered in the media. 
This technique, coined the crashing memory paradigm, has recently been employed to investigate people's "memory" of non-existent footages of a wide range of public events (Granhag, Strömwall, \& Billings, 2003; Jelicic, Smeets, Peters, Candel, Horselenberg, \& Merckelbach, 2006; Ost, Hogbin, \& Granhag, 2006; Ost, Vrij, Costall, \& Bull, 2002b; Wilson \& French, 2006). Ost and colleagues (2002b), for example, found that 44 percent of their participants were willing to report that they had seen television fragments of the 1997 fatal crash of Diana, Princess of Wales, when in fact, no such film material exists. Interestingly, these authors obtained evidence that compliance (i.e., eagerness to please) may be a key factor in explaining why so many people claim to have seen non-existent footage. Similarly, Granhag et al. (2003) showed that a substantial number of Swedish participants (i.e., 38 percent in study 1 and 55 percent in study 2) came to report having seen a non-existent film of the disastrous sinking of the Estonia ferry, in which almost 900 lives were lost. Yet another replication of Crombag et al.'s crashing memories is a study by Jelicic et al. (2006), who asked Dutch undergraduate students whether they had seen footage of the assassination of Dutch politician Pim Fortuyn and if so, whether they could remember any details of it. Sixty-three percent of the participants claimed they had seen the non-existent footage, yet only 23 percent were able to provide details. Interestingly enough, those participants who came up with details of the footage were characterized by elevated levels of fantasy proneness (i.e., a deep and profound involvement in fantasy and imagination; see Lynn \& Rhue, 1988).

The fact that a substantial proportion of individuals are willing to state that they have seen footage of an event that actually does not exist, has sometimes been interpreted as implying that it is relatively easy to induce full-blown pseudo-memories in healthy people. For example, as many participants in the Crombag et al. (1996, p. 102) study gave detailed answers to the follow-up questions, the authors asserted that "[...] apparently these subjects had formed images [...]." However, one could take the stance that the high percentage of participants claiming to have seen the footage does not necessarily mean that all of them actually had pseudo-memories or images (Smeets, Merckelbach, Horselenberg, \& Jelicic, 2005; see for a similar interpretation of results from imagination inflation studies, Scoboria, Mazzoni, Kirsch, \& Releya, 2004). Another explanation for these results could be that participants, due to the highly suggestive context, drew upon general knowledge heuristics rather than pseudo-memories when they answered the interview questions. As well, participants may have been eager to please the researchers and, hence, might have provided socially desirable answers without really believing them. Finally, it might have been the case that some of the participants misinterpreted the critical question and believed that it related to several television images depicting the aftermath of the various events (e.g., the destroyed apartment buildings, the car wreckage). 
Perhaps the most parsimonious explanation for these false reports can be found in the original work by Crombag et al. (1996). They speculated that the misleading suggestions embedded in their questions may have led their participants to come to believe that they saw a television fragment that in fact did not exist. If this holds true, one wonders to what extent manipulating the way in which one formulates the critical question (i.e., varying its specificity or the amount of misleading information conveyed by it) modulates the proportion of participants who claim to have seen the non-existent footage. Using the assassination of the Dutch politician Pim Fortuyn as the target event, the current study addressed this issue by varying the wording of the critical question. Participants were given one of four critical questions. The critical questions differed with regard to their ambiguity and the extent to which they conveyed misleading information (see below). In the current context, ambiguity refers to a question that does not articulate whether it pertains to the incident itself or its aftermath (i.e., images of the body surrounded by a firstaid team and the police). Misleading information refers to the assumption embedded in a question that the footage must exist.

If it is true that previous reports of having seen non-existent footage of a high publicity event (e.g., Crombag et al., 1996; Granhag et al., 2003; Jelicic et al., 2006; Ost et al., 2002b) were shaped by (mis)leading and ambiguous questions, then participants given a crashing memory question that was both vague and misleading would be expected to yield the highest percentage of "yes"-responses. Furthermore, a higher proportion of "yes"-responses in participants given a specific, but misleading question would then be anticipated in comparison to the group that received a specific, but less suggestive question. The group that received a neutral question, in turn, would be expected to produce the lowest percentage of "yes"responses.

We also administered the Memory Characteristics Questionnaire (MCQ; Johnson, Foley, Suengas, \& Raye, 1988), which assesses a wide variety of memory qualities. Note that in the present study, participants who did not endorse false reports of having seen the non-existent footage were asked to imagine having seen such footage. Subsequently, they were asked to provide MCQ ratings for the images they had formed through imagination. One would expect that participants with false reports of the non-existent film would have few problems in retrieving images supporting their false reports. Therefore, it was predicted that participants endorsing the false reports would provide higher MCQ scores than participants who deny to have seen the non-existent footage. Indeed, there are some indications in the literature that fabricated memories are sometimes rated as more vivid and detailed than real memories (e.g., Porter, Yuille, \& Lehman, 1999). 


\section{Methods}

\section{Target event}

Like Jelicic et al. (2006), the current study used the Pim Fortuyn assassination as the target event. Being a famous Dutch politician, Pim Fortuyn was an intriguing right wing representative who had gained national recognition for his opinions on immigration control and the systematic integration of foreigners already residing in the Netherlands. He was the person heading the list of candidates for a new political party called "List Pim Fortuyn" in May 2002, and opinion polls for the upcoming Dutch general elections had shown that his party would gain a considerable number of seats in the Dutch parliament. On May $6^{\text {th }} 2002$, just as he was leaving a radio station where he had given an interview, Pim Fortuyn was shot and killed by animal rights activist Volkert van der G. ${ }^{27}$ The Pim Fortuyn assassination shocked many people both inside and outside the Netherlands and was front page news in national and international newspapers. For many months, the Dutch media extensively covered the murder and its aftermath, thereby also showing images of the dead body. There is, however, no video footage of the moment Fortuyn was shot.

\section{Participants}

Our sample consisted of 120 individuals (49 men and 71 women) selected at random from the community. Their mean age was 38 years ( $S D=12.4$; range: 20-63). The entire test protocol was approved by the standing ethical committee of the Psychology Department of Maastricht University. All participants signed a written informed consent and were asked to report age, gender, and level of education (following Verhage, 1964). Groups (see below) did not differ with respect to age $[F(3,116)=0.21 ; p=.89]$, gender distribution $\left[\chi^{2}(3, N=120)=0.38 ; p=.94\right]$, or level of education $[F(3,116)=0.67 ; p=.56]$. All participants reported they were local residents and lived in the Netherlands at the time Pim Fortuyn was killed.

\section{Materials}

Crashing memory questionnaire. Four sets of questionnaires were developed specifically for this study, each differing only with respect to the critical question. Participants were first required to provide some personal background characteristics, such as age, gender, and educational level. They then were asked two factual questions regarding the assassination of Pim Fortuyn, one probing for the exact date of the Pim Fortuyn assassination, the other querying for the location of the murder. The third question was the critical crashing memory question probing for knowledge of

\footnotetext{
${ }^{27}$ Under Dutch law, it is illegal to reveal the full name of a suspect in the media. As the suspect of the Pim Fortuyn assassination was consistently referred to as Volkert van der G. in Dutch media, this alias was used in the critical questions developed for this study.
} 
the Pim Fortuyn shooting. Participants were randomly assigned to one of the following groups. Participants in the first group received the rather vague question "Did you see the amateur film of the Fortuyn shooting?" (ambiguous group; $n=30$ ), while a second group was given a more restrictive, specific question, namely "Did you see the amateur film of the moment Fortuyn was shot by Volkert van der G.?" (i.e., the specific high-suggestive group; $n=30$ ). A third group was asked a less suggestive variant of the specific suggestive question, which was phrased as follows: "Did you see an amateur film of the moment Fortuyn was shot by Volkert van der G.?" (specific low-suggestive group; $n=30$ ). Finally, participants in the fourth group were queried with a neutral, non-suggestive, open question formulated as "Do you remember whether there was a film of the moment Fortuyn was shot by Volkert van der G.?” (neutral group; $n=30$ ). It should be acknowledged here that this neutral question is different in terms of the decision that has to be made. That is, while the ambiguous, specific high-suggestive, and specific low-suggestive question solicit whether participants remember seeing the footage, the neutral question taps more general knowledge of whether such footage exists. However, throughout the remainder of this article we will use the term neutral to refer to this question. The critical question was answered in a "yes, I remember that film"/ "no, I do not remember such a film" format. Thus, the pertinent questions given to the groups differed in the amount of misleading information and specificity they conveyed.

Finally, if participants indicated having seen the non-existent footage, they were asked to write down in as much detail as possible whatever they could remember from the footage. These data were scored dichotomously $(0=$ without details; $1=$ with details). That is, we focused on whether participants came up with specific details (e.g., shots being fired, Fortuyn collapsing, etc.) that they could not have seen (i.e., for which no actual footage exists). If they did, a 1 was scored. Yet, details derived from what was shown in the media, i.e., details pertaining to the events surrounding the assassination (e.g., the first aid team and police officers, the dead body) were scored as 0 .

Memory Characteristics Questionnaire. The Memory Characteristics Questionnaire (MCQ; Johnson et al., 1988) comprises 39 statements that tap a broad range of memory characteristics. For most statements, participants are required to indicate on a 7-point scale (anchors vary depending on the item) the degree to which they agree with the statement. McGinnis and Roberts (1996) showed that the MCQ consists of 8 subscales. For practical considerations, only the subscales Clarity, Thoughts and Feelings, Sensory Components, Valence, and Frequency of Consideration were administered. The Clarity subscale consists of 5 items related to memory clarity and vividness (e.g., "My memory for the event is: $1=$ sketchy; $7=$ very detailed"), while the Thoughts and Feelings subscale involves 6 items associated with the emotional components of the memory and the implications of the remembered experience (e.g., "At the time, the event seemed like it would have serious implications: $1=$ not at all; $7=$ definitely"). The 4 items related to memory for touch, 
sound, taste, and smell (e.g., "My memory for this event involves sound: $1=$ little or none; $7=$ a lot") constitute the Sensory Components subscale, while the 2 items tapping the magnitude of positive or negative tone or feelings (e.g., "Feelings at the time were: $1=$ negative; $7=$ positive") make up the Valence subscale. The 2 items related to how frequently an individual has thought or talked about the specific event (e.g., "Since it happened, I have thought about this event: $1=$ not at all; $7=$ many times"), comprise the Frequency of Consideration subscale.

\section{Procedure}

Data collection took place about 36 months after the Fortuyn assassination. Clients of a local shopping venue were approached and asked whether they would like to participate in a study on memory for emotional events. The investigators continued to approach individuals until all four groups comprised 30 participants. In total, about 200 potential participants had to be approached. Participants gave written informed consent and were then asked to carefully read and complete three questionnaires. First, participants were randomly given one of four variants of the crashing memory questionnaire concerning the Fortuyn assassination. Next, they filled out the MCQ and also the Gudjonsson Compliance Scale (Gudjonsson, 1989), which will not be considered here. Afterwards, participants were debriefed and thanked for their participation.

\section{Results}

Background characteristics and gender distribution for each of the four subgroups can be found in Table 8.1. Sixty-three percent of our participants in the ambiguous group, 30 percent in the specific high-suggestive as well as the specific lowsuggestive group, and 27 percent in the neutral group claimed to have seen the non-existent footage. Pearson Chi-Square Exact test (2-sided) was used to evaluate group differences in the frequency of "yes" responses (i.e., falsely claiming to have seen the footage). This yielded a significant effect of question type $\left[\chi^{2}(3, N=120)\right.$ $=11.48 ; p=.009$; Cramer's $V=.31$, representing a medium effect size]. Meanwhile, there were no group differences with respect to the frequency of correct answers to both the first $\left[\chi^{2}(3, N=120)=0.65 ; p=.91\right.$; Cramer's $\left.V=.07\right]$ and the second $\left[\chi^{2}(3, N=120)=3.73 ; p=.32\right.$; Cramer's $\left.V=.18\right]$ factual question. Thus, group differences in factual knowledge about the assassination do not account for group differences in false reports.

Mean MCQ ratings for "yes"- and "no"-respondents can be found in Table 8.2. To find out whether "yes"-respondents differed from "no"-respondents in terms of memorial qualities, we conducted a MANOVA on each of the 5 MCQ subscale ratings. Box's $M$ test for homogeneity of variance was found to be non- 
significant [Box's $M=15.36 ; F(15,34630)=0.97 ; p=.48]$ and, therefore, homogeneity was assumed. "Yes"-respondents did not differ from "no"-respondents on the MCQ with respect to the Clarity subscale $[F(1,118)<.01 ; p=.99]$, the Thoughts and Feelings subscale $[F(1,118)=1.56 ; p=.21]$, the Sensory Components subscale $[F(1,118)=0.05 ; p=.82]$, the Valence subscale $[F(1,118)<.01 ; p$ $=.95]$, or the Frequency of Consideration subscale $[F(1,118)=0.09 ; p=.77] .{ }^{28}$

Table 8.1. Means (standard deviations) for background characteristics of the participants, the proportion of participants claiming to have seen the footage, and the proportion of participants providing details for each of the four subgroups ( $n=30$ per group).

\begin{tabular}{|l|c|c|c|c|}
\hline & Ambiguous & $\begin{array}{c}\text { Specific high- } \\
\text { suggestive }\end{array}$ & $\begin{array}{c}\text { Specific low- } \\
\text { suggestive }\end{array}$ & Neutral \\
\hline Age (years) & $39.0(12.0)$ & $37.8(11.9)$ & $36.6(13.4)$ & $38.6(12.9)$ \\
\hline Male/female ratio (\%) & $43.3 / 56.7$ & $43.3 / 56.7$ & $36.7 / 63.3$ & $40.0 / 60.0$ \\
\hline Educational level (max=7) & $5.83(1.1)$ & $6.00(0.9)$ & $6.03(0.8)$ & $5.73(0.9)$ \\
\hline Proportion “yes"-responses (\%) & 63.3 & 30.0 & 30.0 & 26.7 \\
\hline Proportion providing details (\%) & 33.3 & 20.0 & 23.3 & 6.7 \\
\hline
\end{tabular}

Note: ${ }^{\mathrm{a}} p<.05$

We also asked participants who claimed to have seen the non-existent footage whether they could remember specific details of the footage. Overall, 20.8 percent of the entire sample came up with such details. Breaking this percentage down for each of the 4 subgroups, we found that 33 percent in the ambiguous group, 20 percent in the specific high-suggestive group, 23 percent in the specific lowsuggestive group, and 7 percent in the neutral group provided details $\left[\chi^{2}(3, N=\right.$ $120)=6.62 ; p=.10 ;$ Cramer's $V=.24]$. Participants providing details had similar scores on the MCQ subscales as participants who did not report details [Box's $M=$ 8.46; $F(15,7647)=0.52 ; p=.93$; all $F$ s $(1,118)<0.74$; all $p$ ’s $>.39] .{ }^{29}$

\footnotetext{
28 One could speculate that if varying the question format would lead to a manipulation of source monitoring criteria, these analyses might yield a different pattern of results had we looked within each of the 4 groups separately. Therefore, we conducted MANOVA's on the 5 MCQ subscale ratings comparing yes- and no-respondents within each group. Results were highly similar in that for all 4 groups, MCQ ratings did not differ between participants endorsing the false reports and participants who did not make such false reports (all $p$ 's $>.05$ ).

${ }^{29}$ Even when we looked at the 4 groups separately, no differences on MCQ ratings were found between participants who did and those who did not provide false details (all $p$ 's $>.05$ ).
} 
Table 8.2. Means (standard deviations) for MCQ ratings of the participants reporting having seen the footage $(n=45)$ and participants without such claims $(n=75)$.

\begin{tabular}{|l|c|c|}
\hline MCQ & "Yes"-respondents & "No"-respondents \\
\hline Sensory components & & \\
\hline Clarity & $21.93(3.68)$ & $7.09(3.61)$ \\
\hline Valence & $4.24(1.68)$ & $21.68(5.71)$ \\
\hline Thoughts and feelings & $29.64(6.84)$ & $4.27(1.90)$ \\
\hline Frequency of consideration & $8.13(2.24)$ & $28.19(5.76)$ \\
\hline
\end{tabular}

Note: MCQ is Memory Characteristics Questionnaire.

\section{Discussion}

The present study sought to investigate whether it is the ambiguous or suggestive nature of the assessment procedure that serves as the crucial antecedent of reports of non-existent film fragments of tragic public events (Crombag et al., 1996; Granhag et al., 2003; Jelicic et al., 2006; Ost et al., 2002b). The main results of the present study can be summarized as follows. First, a substantial number of participants (37.5 percent overall) erroneously reported having seen footage of the Pim Fortuyn assassination. Second, the frequency with which these reports were made was related to the way in which participants were interviewed. That is, depending on the precise condition, the proportion of participants claiming to have seen the footage varied between 27 percent and 63 percent. MCQ ratings did not differ between participants reporting to have seen the footage and those who did not, or between participants providing details of the footage and participants who did not report such details.

The fact that many people are willing to falsely report having seen nonexistent footage replicates previous findings by Crombag et al. (1996), Ost et al. (2002b), Granhag et al. (2003), and Jelicic et al. (2006). However, in line with our prediction that these reports are shaped by misleading and/or ambiguous questions, our data show that the rate at which people endorse having seen the footage depends on the way they are queried. More precisely, we found that when participants were asked an unambiguous question (i.e., specifically conveying the message that the footage they were queried about relates to the instant where Pim Fortuyn was shot), the proportion of them making false claims was significantly lower compared to that in the group given an ambiguous question. In our view, this suggests that at least a subgroup of participants in the ambiguous group that made false reports merely affirmed having seen images of events surrounding the Fortuyn murder. 
The fact that false report rates were related to how the questions were formulated also shows that language to some degree interacts with subsequent "memory" reports. Our findings concur with, for example, the scholarly work by Loftus and Palmer (1974). These authors noted that participants who had been shown films of automobile accidents gave lower speed estimates when they were questioned using the verbs collided, bumped, contacted, or hit than when the verb smashed was used. Moreover, these authors found that participants who were questioned using the verb smashed were more likely to falsely report having seen broken glass than when any of the other verbs were used. Further support for the language-memory report interaction comes from the work by Grice (1989). This author pointed out that human communication rests on the fundamental assumption that the questioner provides information that is clear (i.e., the maxim of manner) and trustworthy (i.e., the maxim of quality). Indeed, the mere fact that an individual is probed for footage of a public event presupposes that the footage actually exists. Thus, in our view, it is plausible that even the neutral question misled some participants. Many studies have found that about 30 percent of any sample is willing to offer an opinion on fictitious issues (see Schwarz, 1999) and this percentage fits nicely with the rate of 27 percent in the neutral group who said they had seen the non-existent footage.

It is important to note that the original study by Crombag and colleagues used a specific question (i.e., "Did you see the television film of the moment the plane hit the apartment building?"; see Crombag et al., 1996, p. 99) to investigate whether their participants would claim to have seen the footage of the El-Al crash. The authors found that 55 percent (study 1) and 66 percent (study 2) of their participants mistakenly claimed to have seen the footage. However, it is conceivable that at least a subgroup of these individuals tried to be cooperative and mistook the animated reconstruction film that was extensively used by the media to cover the aftermath of the crash for real television footage of the actual crash. We acknowledge, though, that our interpretation is speculative and should not be taken as a comprehensive account of the Crombag et al. findings.

Although the current study showed that an ambiguous question can potentiate false report rates, it failed to find differences for these rates between specific high-suggestive, specific low-suggestive, and neutral question formats. Accordingly, our data somewhat contradict the notion that crashing memory reports are shaped by the suggestiveness of the probe. Perhaps, then, it is not so much the suggestiveness of the question, but rather the suggestibility of the respondent that is of importance here. Another possibility is that our manipulation of the suggestiveness of the critical question was too subtle and, hence, resulted in non-significant differences between the specific high-suggestive, specific low-suggestive, and neutral groups. The fact that we were unable to find differences between the specific highand specific low-suggestive group is at variance with, for example, work by Loftus and Zanni (1975). These authors had their participants view a film of a motor ve- 
hicle accident, after which they were asked either "Did you see a broken headlight?" or "Did you see the broken headlight?" Loftus and Zanni (1975) found that participants were more likely to answer "yes" when the question was posed with the definite article "the" than the indefinite article "a". Future research should explore these issues in more detail.

In light of the fact that the current data was obtained using a forced choice answer format, it is interesting to note that previous studies have shown that report option has a substantial effect on memory accuracy (see, for example, Koriat \& Goldsmith, 1994, 1996; Roebers \& Fernandez, 2005; Schwarz, 1999). In general, forced choice formats yield lower accuracy rates than open-ended questions and other answer formats. Thus, we can only speculate as to what would happen when studies like the current were to include answer options such as "I'm not sure" or "I don't know" (e.g., Roebers \& Fernadez, 2005), or allowing participants to refuse to respond (e.g., Koriat \& Goldsmith, 1996). In our view, this would lead to increases in memory accuracy and, hence, fewer claims of having seen the non-existent footage.

An intriguing aspect of the current data concerns the proportion of participants providing details that they could not possibly have witnessed. Twenty-one percent of the participants in the present study confabulated at least one specific detail related to the event at hand. Most likely, these participants tried to reconstruct what they believe happened during the event from the information that was communicated in the press. Our results concur with those of Jelicic et al. (2006), who found that 23 percent of their participants provided details of a non-existent film. It is worthy of note that Jelicic and co-workers found that participants willing to provide details of non-existent footage were characterized by elevated fantasy proneness scores. Their finding is reminiscent of a study by Platt, Lacey, Iobst, and Finkelman (1998) in which a connection was noted between absorption - which is a close cousin of fantasy proneness - and spontaneous memory distortions in participants' flashbulb memories of the O. J. Simpson trial. Similarly, Eisen and Carlson (1998) found that heightened levels of absorption predicted participants' tendency to give in to misleading questions about the peripheral details of a staged event. Thus, a deep and profound involvement in fantasy and imagination (i.e., fantasy proneness) may increase participants' willingness to envision particular aspects of how an event must have taken place. Investigating the precise role that fantasy proneness plays in the reconstruction of these details could prove fruitful to future research. Also note that the proportion of participants falsely conveying details corresponds nicely to the percentage of participants who come up with false beliefs or pseudo-memories typically found in pseudo-memory studies (e.g., Porter et al., 1999; Hyman \& Billings, 1998). Evidently, a host of factors operating in these latter studies (e.g., repeated questioning, ruminating about the critical event between sessions) may be held responsible for the obtained false reports. 
Regarding the MCQ ratings, the present study replicated the findings by Ost et al. (2002b) in that the MCQ ratings of the "yes"-respondents were not significantly different from those of "no"-respondents. Neither did the MCQ ratings differ between participants providing details of the footage and participants who did not report such details. These results admit of at least two interpretations. One interpretation would be that the MCQ does not serve well to distinguish imagined from false reports as elicited by the crashing memory paradigm. Alternatively, perhaps the most straightforward way of accounting for the MCQ data would be in terms of Fuzzy-Trace Theory (Brainerd \& Reyna, 2002; Reyna \& Brainerd, 1995). This theory holds that during encoding verbatim and gist traces are formed in parallel. The crux of the matter is that there is a differential survival rate for verbatim and gist traces, with gist traces showing superior memorability and accessibility over time (e.g., Brainerd \& Reyna, 2004). One could therefore argue that the time lag of approximately 3 years between the critical event (i.e., the Fortuyn killing) and the memory interview was so long that verbatim memory traces were subjected to considerable decay, leaving mainly gist traces intact for future attempts at retrieval. If participants endorsing the false reports primarily drew upon gist information (e.g., Fortuyn being shot and killed) in answering the MCQ items, then it is not surprising that their MCQ ratings are similar to those of participants who did not endorse false reports.

Some notes on the potential limitations of the present study are in order. First, our study relied on a community sample that voluntarily took part in our survey. Although implausible, it cannot be entirely ruled out that those individuals who were willing to participate are in some way different from people who declined to participate (e.g., in assertiveness). Second, a limitation of the crashing memory paradigm is that there is a possibility that some participants may have come to falsely believe in having seen the non-existent footage prior to being interviewed about it (i.e., in the interval stretching from the event itself to being questioned). Thus, it is unfeasible to determine with any degree of certainty whether those participants making false reports do so in response to being interviewed or that they only endorse a false belief they had developed in advance. Another drawback of the current study concerns the individual differences measures that were examined. For practical consideration, we included only some of the MCQ subscales. Future studies might benefit from additionally including scales intended to evaluate participants' tendency to provide socially desirable answers (e.g., the Social Desirability Scale; Crowne \& Marlow, 1964) to investigate whether a response bias may account for some of the false reports elicited by the crashing memory paradigm. Moreover, the precise role fantasy proneness plays in reporting details that could not have been witnessed, deserves further exploration.

Summing up, this study provides the first evidence that crashing memory reports may be related to how the questions are phrased. Future research should take these findings into account when constructing questionnaires intended to investi- 
gate people's accounts of specific events. The fact that 27 percent of the participants given a neutral question claimed to have seen the non-existent film, underscores the fact that misleading and ambiguous questions are not the sole or even the most important explanation for the results previously obtained through the crashing memory paradigm. We believe that in these participants, it is the question per se that fuels distorted reports. Conducting extensive post-experimental interviews could shed further light on this issue. 
General Discussion 


\section{Introduction}

The present dissertation was set out to further investigate the functions of acute stress and cortisol elevations in memory recollections in general, and in the development of pseudo-memories in particular. To this end, we first examined the effects of acute psychosocial stress on memory for emotional and neutral stimuli and spontaneously occurring commission errors (i.e., list intrusions). Subsequently, in two studies we examined whether acute stress and/or cortisol elevations might potentiate false recollections as elicited by the Deese-Roediger-McDermott (DRM) paradigm. Furthermore, we looked at whether exposure to an acute stressor might change memory for the source of information (i.e., source monitoring performance). Finally, to test the hypothesis that delayed recall of childhood trauma can be explained by neuroendocrine alterations of the hypothalamic-pituitary-adrenal (HPA) axis, we investigated the daily cortisol output in women harvesting repressed, recovered, or continuous memories of childhood sexual abuse (CSA), and compared them with non-traumatized controls.

Moreover, this dissertation reviewed pseudo-memory research that has been carried out to date and highlighted some limitations of the research paradigms that have been used. As well, we experimentally investigated an alternative explanation for one of these paradigms demonstrating high rates at which people report having seen images of high-publicity events that, in fact, do not exist (i.e., the crashing memory paradigm).

\section{Stressing memories: Stress and cortisol as determinants of false recollections?}

From the time when Sir Frederic Bartlett (1932) published his seminal work on human memory performance, research on memory has gradually shifted from a focus on accurate reproduction of past experiences (Koriat, Goldsmith, \& Pansky, 2000) to the broad consensus among psychologists about the inherently reconstructive nature of episodic memory (e.g., Roediger, 1996; Schacter, 1995). Experimental studies on the fallibility of memory have demonstrated its proneness to distortions and confabulations. That is, individuals may remember events or facts differently from how they originally occurred or may even remember events that did not take place at all.

In her pioneering work, Elizabeth Loftus demonstrated that misleading postevent suggestions may cause memory distortions (for a recent overview, see Loftus, 2005). In a typical misleading information study, participants are first exposed to a videotaped or staged sequence of events, and then asked questions about these events, some of which contain suggestions of incidents that never oc- 
curred. Loftus and co-workers repeatedly found that people will falsely recall or recognize some of the suggested events as authentic events. Similarly, work by Henry Roediger III and his associates (e.g., Roediger \& McDermott, 1995) building on early research of Deese (1959) provides another potent illustration of false recollections. In what is now know as the DRM paradigm, lists of semantically associated words, all of which converge on a single non-presented theme word, are presented to participants. In a subsequent recall or recognition task, the theme word is often falsely recollected by the majority of participants. In addition to the misleading information and the DRM paradigm, other paradigms have been developed that are intended to investigate the implantation of entire autobiographical pseudomemories (e.g., Davis \& Loftus, in press; McNally, 2003; Ost, 2006; Ost, Foster, Costall, \& Bull, 2005), often fostered by parental misinformation. In general, studies that are set out to elicit autobiographical pseudo-memories do not confront participants with misleading information, but instead result from participants' deductive inferences or internally created constructs that they subsequently mistake for external events (i.e., source monitoring errors, see Johnson, Hashtroudi, \& Lindsay, 1993). Loftus and Pickrell (1995), for example, exposed their participants to written information on two authentic childhood events of which the participants' parents provided the details. A third event that was described, however, pertained to an event of which the parents were sure it never happed to their child. Loftus and Pickrell (1995) found that over the course of three weeks during which 'memory interviews' were performed, 25 percent of the participants erroneously came to believe part or all of the falsely suggested event.

Despite forceful research from the past decade, it remains largely unknown what factors contribute to the formation of pseudo-memories, or which type of person may be particularly vulnerable to develop these false recollections. Individuals with executive dysfunctions leading to poor source monitoring (Johnson et al. 1993), seem to be particularly susceptible to false recollections (e.g., Parkin, 1997; Parkin, Bindschaedler, Harsent, \& Metzler, 1996; Peters, Jelicic, \& Merckelbach, 2006; Peters, Jelicic, Verbeek, \& Merckelbach, in press; Schacter, Koutstaal, \& Norman, 1997). In a similar vein, being exposed to an acute stressor (i.e., Trier Social Stress Test or TSST) has been shown to boost pseudo-memories in a DRM paradigm (Payne, Nadel, Allen, Thomas, \& Jacobs, 2002). Although Payne et al. (2002) did not measure cortisol responses to the stressor, it is a distinct possibility that excessive cortisol levels were responsible for the increased vulnerability to pseudo-memories.

A review of the pseudo-memory literature (see Chapter 7) showed that quite a number of studies allegedly investigating pseudo-memories, in fact have shown nothing more than increased confidence that some fictitious childhood event might have happened. Other work adds to this in that many studies have led people to falsely believe that some aversive event in fact did happen in their childhood. Fewer studies, however, have really succeeded in implanting entire, full- 
blown pseudo-memories. Of late then, other authors have begun to appreciate similar points of view and have also vented the idea that increased likelihood, false beliefs, and pseudo-memories are different phenomena and that for many studies, it is uncertain to what extent they have surpassed the level of false belief (e.g., Loftus \& Bernstein, 2005; Ost, 2006). With the exception of work by Bernstein and colleagues (e.g., Bernstein, Laney, Morris, \& Loftus, 2005a, 2005b) and studies on false confessions (e.g., Candel, Merckelbach, Loyen, \& Reyskens, 2005; Kassin \& Kiechel, 1996; Horselenberg, Merckelbach, \& Josephs, 2003; Horselenberg, Merckelbach, Smeets, Franssens, Peters, \& Zeles, 2006; Redlich \& Goodman, 2003), research to date has not looked at whether all sorts of pseudo-memory manipulations also bear consequences in that people will act based on their false recollections. In looking critically at pseudo-memory studies, some problematic features pertaining to the crashing memory paradigm and the interpretation of results stemming from studies employing this paradigm were highlighted. In the final experimental chapter of this dissertation, we investigated whether the way in which people are probed for certain knowledge of emotionally charged events, makes people more or less likely to endorse false reports.

\section{Summary of research findings}

Previous research has shown that acute stress impairs declarative memory performance (e.g., Kirschbaum, Wolf, May, Wippich, \& Hellhammer, 1996b), but that this effect varies as a function of the valence of the to-be-remembered memory material (e.g., Abercrombie, Speck, \& Monticelli, 2006; Tops et al., 2003). Chapter 3 reported on a study that further investigated the effects of acute stress on declarative memory performance as a function of stimulus valence. Results of this study showed that stressed participants exhibited impaired recall of neutral words, but that recall of positive emotional and negative emotional words remained unaffected. Participants who were stressed also made more commission errors (i.e., extra-list intrusions) than did control participants. Moreover, for the stressed but not the control participants, post-stress performance on working memory measures was significantly correlated with correct recall of neutral words.

Four other studies have been carried out regarding the link between stress, pseudo-memories, source monitoring errors that may underlie pseudo-memories, and delayed disclosure of traumatic childhood memories. The two studies described in Chapter 4 attempted to replicate findings by Payne and co-workers (2002) showing that acute stress may lead people to falsely recall or recognize nonpresented words. After having been exposed to an acute laboratory stress task, participants in both study 1 and study 2 falsely recalled and recognized the nonpresented critical lures at similar rates as non-stressed control participants did. Study 2 additionally showed that even when we looked at those participants who 
had the highest cortisol elevations in response to the stressor, they did not demonstrate elevated levels of false recall or false recognition in comparison to low cortisol responders or controls. Furthermore, no significant correlations between false recall and recognition rates and delta increases in cortisol were revealed.

Subsequently, we examined whether acute stress or the ensuing cortisol elevations could alter source monitoring performance in an internal-internal source monitoring task (see Chapter 5). The results of this study indicated that participants exposed to the stressor exhibited better correct identification of the source of their memories than non-stressed controls. In addition, relative to controls, stressed participants were more accurate at discriminating between targets and distracters when the number of errors that were made was taken into account. This would imply that if anything, exposure to acute stress renders people less likely to confuse the source of their memory and, hence, for pseudo-memories to arise. Finally, source monitoring performance did not differ between low and high cortisol responders, suggesting that the magnitude of the cortisol response does not directly influence the effects of acute stress on source monitoring performance.

In Chapter 6, we explored the hypothesis that delayed recall of CSA can be explained by neuroendocrine alterations of the HPA axis. To this end, the morning cortisol response and diurnal cortisol pattern of women reporting continuous, repressed, or recovered memories of CSA were compared to non-abused control participants. Contrary to what one would predict on the basis of popular theories from the recovered memory literature, no abnormalities in cortisol patterns of women who had repressed, recovered, or continuous CSA memories were found. That is, neither the awakening rise nor the diurnal pattern in cortisol differed between CSA groups and controls, with all four groups showing a straightforward morning response and a typical diurnal rhythm of cortisol secretion.

Over the past decades, there has been a growing body of literature purporting to examine pseudo-memories. As argued in Chapter 7, not all research paradigms that have been employed over the years have succeeded in implanting full-blown (i.e., rich) pseudo-memories. Also included in the review of Chapter 7 were studies that used the so-called crashing memory paradigm in which individuals claim to have seen non-existent footage of high-publicity events. Chapter 7 suggested that, perhaps, the most parsimonious explanation for the high percentage of participants who generally make these false reports is the ambiguity and/or specificity of the critical crashing memory question that is put to them.

Chapter 8 explored whether people who reported having seen non-existent video fragments of the killing of famous Dutch politician Pim Fortuyn, did so because they were being misled by the ambiguity of the non-sensical question that was posed to them. Results of this study showed that while 38 percent of all participants erroneously reported having seen the footage of the Pim Fortuyn assassination, the rate at which people endorse false reports in part depends on the ambiguity of the crashing memory question. Moreover, the number of participants 
falsely reporting having seen the non-existent video footage was greatly reduced by explicitly mentioning the boundaries of the critical incident for which they were probed.

\section{Contradictions and limitations}

The hypothesis that acute stress and cortisol elevations may render people vulnerable to pseudo-memories produced somewhat mixed findings. That is, while levels of spontaneously occurring, extra-list commission errors were higher for stressed participants relative to controls in the study presented in Chapter 3, no effect of stress or high cortisol levels were found on levels of false recall or false recognition as elicited by the DRM paradigm (see Chapter 4). Furthermore, the studies of Chapter 4 did not show elevated levels of commission errors in a free recall task following acute stress. These contradictory results might be explained by looking at the interval between list learning (i.e., encoding) and the beginning of the free recall tasks. While both studies in Chapter 4 used an immediate free recall task, the study described in Chapter 3 employed a retention interval of 30 minutes before free recall performance was assessed. As forgetting increases over time, especially directly after learning, the longer retention interval in Chapter 3's study may have provided optimal conditions for commission errors to occur. Overall, it seems safe to state that although the evidence is somewhat equivocal, acute stress does not seem to potentiate commission errors or false recall/recognition in the DRM paradigm.

Furthermore, it was argued that not all of these paradigms are equally fit to make statements about whether or not they have succeeded in eliciting pseudomemories, and whether they may have behavioural consequences. Finally, the study on crashing memories (Chapter 8) underscores the importance of phrasing questions that are intended to examine individuals' specific memories in such a way that the answers truly reflect what is supposed to be under investigation (see Schwarz, 1999, for an excellent overview on the importance of question design and wording).

A number of limitations of the studies described throughout this dissertation deserve to be mentioned. First of all, except for the study that investigated HPA axis functioning in women with recovered, repressed, and continuous memories of CSA (Chapter 6) and the crashing memory study (Chapter 8), the current studies relied on samples of undergraduate students. Whereas student populations are appealing in that they represent well-balanced, homogenous groups reducing unwanted variance, their memory may not be representative of the general population. Also, the studies investigating acute stress effects on memory performance reported in this dissertation used a psychosocial stressor (i.e., TSST) to elicit high levels of stress. While the TSST has been shown to reliable elicit significant HPA 
stress responses, it does not rule out the possibility that other types of stressors (e.g., physical stressors) may lead to different results. Additionally, one could argue that although the TSST elicits significant cortisol responses, the magnitude of the response is simply too small to bring forth large effects on (pseudo-)memory tasks, and that using a protocol in which glucocorticoids (GCs) are administered orally to obtain high cortisol levels would have been more desirable. However, as evidenced by the fact that chronic stress in patients suffering from posttraumatic stress disorder (PTSD) is associated with reduced rather than elevated levels of cortisol (e.g., Yehuda, 2002), high cortisol levels should not be equated with stress. Therefore, both types of studies (i.e., those using acute stressors and those relying on cortisol administration) seem warranted. Finally, it is also true that we only looked at cortisol levels and have thus ignored the potential noradrenergic effects of acute stress on memory performance.

Another point that deserves to be mentioned is that we, following work by Payne et al. (2002), used the DRM paradigm to elicit false recall and false recognition of (non-presented) critical lure words. This paradigm has recently been the subject of some debate. Specifically, Pezdek and Lam (in press) have argued that the reasons why the DRM paradigm has so extensively been used in pseudomemory research, are well-developed theoretical underpinnings of the DRM effect and the relative ease with which it can be implemented. While this might well be true, Pezdek and Lam also argue that most pseudo-memory research (particularly studies that used the DRM paradigm) should not be generalized to real world settings (for a similar argument, see Freyd \& Gleaves, 1996). Though Pezdek and Lam have a point in that laypeople as well as some memory researchers tend to overgeneralize research findings (also see Chapter 7 of the present dissertation), it should be noted that this line of research has substantially advanced our knowledge of autobiographical memory (for a sound rebuttal of the Pezdek \& Lam criticisms, see Wade et al., in press). In addition, it might be worth mentioning that a number of DRM studies (e.g., Payne, Elie, Blackwell, \& Neuschatz, 1996; Roediger \& McDermott, 1995) had their participants judge whether they could actually remember the specific instance when the critical lures were presented, or whether they merely thought they were presented (i.e., knowing). In general, these studies have demonstrated that the majority of people actually claim to remember hearing or seeing the critical lures in the list learning phase (e.g., Payne et al., 1996; Roediger \& McDermott, 1995; but see Mather, Henkel, \& Johnson, 1997). Thus, at least for the main part of these falsely recalled or falsely recognized critical lures, it might seem justified to label them pseudo-memories in the sense that they are accompanied by instances of detailed remembering.

Despite these limitations, the present results may have some implications for court room settings. In particular, they imply that if defendants or eyewitnesses (or expert witnesses speaking for them) testify in court that they have poor memory or even total amnesia for the event(s) they experienced because they found them very 
upsetting and highly stressful (e.g., Cima, Merckelbach, Nijman, Knauer, \& Hollnack, 2002; Cima, Nijman, Merckelbach, Kremer, \& Hollnack, 2004; Kopelman, 1995; Van Oorsouw, 2006), such statements should be treated with some caution. It seems that stressful situations may somewhat impair memory for the neutral, more peripheral details of the experience, but that these impairing effects are not of such magnitude that they turn these individuals amnesic for the experience. Moreover, stress may actually be protective of memory for the more central and highly emotional details. And even when high levels of stress hormones (e.g., cortisol) released during the stressful experience might lead people to forget (or at least not report) certain minor details, this does not directly imply that stress is also adverse in terms of eliciting more pseudo-memories. In other words, it would be illadvised to claim that high levels of stress imply that someone is unreliable in his/her statements.

\section{Reconstruction: Adaptive or maladaptive?}

As stated previously, there is a general agreement that human memory is inherently reconstructive in nature. Upon hearing the term "reconstructive", the majority of people are inclined to deduce that memory hence must also be highly fallible. Indeed, research has shown that not only do people tend to forget as years go by, but that distortions and fabrications may also slip into our memories. Some authors go as far as to state that memory is inherently unreliable. ${ }^{30}$ Moreover, one might even contend that our everyday memory has received some negative connotations in that people tend to think that they forget a lot (or at least too much), that they may be remembering things differently from how they occurred, but also that pseudomemories may develop as we try to reconstruct our memories.

This "dark side" to our memory needs to be presented in a balanced way. For one thing, the reconstructive nature of human memory is benign in that it provides the opportunity to generalize (Riccio, Rabinowitz, \& Axelrod, 1994). In its most basic, Pavlovian form, generalization refers to a tendency to respond to stimuli other than the original stimulus and a propensity for diminished responsiveness as a function of decreased similarity between the original and the novel stimulus. It is critically important to note, however, that generalization gradients tend to level out in the long run. This has everything to do with the fact that discriminability among stimuli lessens over time. ${ }^{31}$ Specifically, forgetting of stimulus attributes (i.e., par-

\footnotetext{
30 An example of such an author would be Hugo Münsterberg (1908), the first psychologist who wrote about eyewitness memory and concluded that eyewitnesses are often chaotic and confused. Another good example would be Haber and Haber (2000), who in this respect wrote about the "tyranny of the eyewitness" (p. 1058).

31 An interesting parallel can be drawn between this decreased memory for stimulus attributes resulting in increased generalization, and the (parallel) encoding of verbatim and gist traces as predicted by
} 
ticular characteristics of an experience) causes one to detect less individuating features of a past experience, and thus makes generalization from past experiences to new contexts more likely. Put another way, people learn from their past experiences and apply the acquired knowledge in new contexts increasingly more as time goes by. Thus, generalization is central to learning and is functional in that people may act rapidly based on their prior knowledge of comparable situations.

This, of course, is especially important under stressful circumstances in which people are given only limited time to decide how to react, based upon their past experiences. An example might clarify this. Suppose you are a novice skydiver and that on one of your parachute jumps, you find yourself in the situation that your parachute fails to open in mid-air. As you are approaching the ground at high speed, you might want to think back at your training days and remember what you should do next. Surely, at that point you do not want to remember the exact details of your training experiences (e.g., the various reasons why a parachute might not open and how you dealt with them in those specific circumstances). Rather, the situation requires you to act as quickly as possible and generalize your past experiences to the present situation so that you immediately know what to do. So, the ability to make generalizations and apply knowledge to new situations is highly important, especially in stressful, threatening situations.

The implication of this is that the memory effects of stress reported in the literature as well as in the current dissertation, may not be replicated when longer retention intervals are used under otherwise identical circumstances (for review, see Riccio, Ackil, \& Burch-Vernon, 1992). At the very least, this draws attention to the potential importance of the often haphazardly chosen retention intervals in determining the effects of acute stress or cortisol on memory performance. Future research should delineate the precise effects that varying retention intervals may exert on the relationship between acute stress and cortisol on memory performance.

\section{An empirical interlude}

As mentioned in Chapter 1, research on the effects of acute stress and/or GCs on declarative memory has yielded a wide range of complex and diverse findings. Though several variables (e.g., valence, sex, time of day) have been identified as modulating the links between acute stress and memory performance, the precise nature of the effects remains unclear. Thus, a comprehensive framework that can account for these contradictory findings is warranted. Recently, Joëls, Pu, Wiegert,

Fuzzy-Trace Theory (FTT; Brainerd \& Reyna, 2002; Reyna \& Brainerd, 1995). More specifically, FTT states that verbatim and gist traces are not remembered equally well over time, with gist traces becoming more accessible over time. 
Oitzl, and Krugers (2006) have made a first attempt to construct such an accommodating framework. These authors proposed that stress will only enhance memory performance when 2 conditions are met: Firstly, exposure to stress is experienced in the context and around the time of learning and, secondly, the brain regions that are impacted by the stress hormones released during stress exposure should be the same as those stimulated by the memory task. More specifically, stress will only promote learning when the stress is context-related to the memory material, such as is the case when an individual is stressed due to an upcoming exam and learns the subject matter while being stressed. Moreover, the memory enhancing effect will only be apparent when in addition to this, stress impacts on the same brain regions as the task at hand, such as when the psychological stress associated with exams impacts upon the hippocampus and the recall task (i.e., exam) also probes for hippocampal-dependent knowledge (e.g., factual knowledge, but not procedural memory).

Recently, we conducted a preliminary study specifically designed to test the theory of Joëls et al. (2006). In short, our aim was to determine whether exposure to a psychosocial stressor may indeed prove to be beneficial to performance on declarative memory tasks that are context-related to the applied stressor. To this end, we recruited 52 healthy Maastricht University undergraduates (13 men, 39 women). ${ }^{32}$ Participants were randomly assigned to one of two stress groups, or a no-stress control group. ${ }^{33}$ In the first group $(n=16)$, participants were exposed to a modified version of the TSST in which they had to perform a 5 minute mental arithmetic task and engage in a 6 minute free speech about their personality while standing in front of a live audience and being videotaped (i.e., the personalityrelated stress group). Similarly, participants in the memory-related stress group $(n=$ 18) also had to perform the modified TSST, but were asked to give a speech concerning the quality of their memory. Participants in the control group $(n=18)$ were shown an emotionally neutral video fragment of a Walt Disney animation film (i.e., filler task). Both TSST's and filler task were equal in duration, and stressfulness of these tasks was determined both subjectively (i.e., participants completed the POMS, the Profile of Mood States, before and after the TSST or filler task) and objectively (i.e., by continuously measuring their heart rate using Polar ${ }^{\circledR}$ portable transmission devices).

At the end of the TSST or filler task, participants were required to listen to 2 word lists of 12 words each, with one list consisting of memory words (e.g., concentrate, knowledge, intellect) and the other containing personality words (e.g.,

\footnotetext{
32 Participants were excluded from the study in case they suffered from endocrine disorders, cardiovascular diseases, or other severe medical illnesses (e.g., fybromyalgia), and were refrained from eating, drinking, smoking, and heavy exercise at least 1 hour prior to the experiment proper.

${ }^{33}$ Groups did not differ with respect to mean age, proportion men versus women, or smoker/nonsmoker ratio.
} 
serious, anxious, modest). ${ }^{34}$ Presentation order of the word lists was counterbalanced within and across groups, and lists were presented on two successive learning trials. Participants were explicitly told that their memory for the words would be tested immediately after presentation of the word lists. Following presentation, a free recall task was administered and participants were asked to return 24 hours later for a surprise delayed recall test. To reduce the likelihood that participants would rehearse the word lists, they were told that their heart rate measurements and recall test would be scored, and that their performance would be discussed the next day. No mention of an upcoming memory test was made. When they returned 24 hours later, a delayed free recall test probing for words from both lists was administered. None of the participants indicated that they had expected a delayed recall test.

Results from this study can be summarized as follows. First, participants were subjectively stressed in both the personality-related TSST as well as the memoryrelated TSST, as indexed by mean increases in POMS scores (personality-related stress group $\mathrm{M}=8.69, \mathrm{SD}=3.03$; memory-related stress group $\mathrm{M}=5.17, \mathrm{SD}=$ 2.50), while a decrease was noted for the control group (control group $\mathrm{M}=-1.94$, $\mathrm{SD}=2.58)[\mathrm{F}(2,49)=4.02 ; \mathrm{p}=.02]$. Mean heart rate responses were averaged over 5 minute intervals for between-group analysis. ${ }^{35}$ Increases in mean heart rate for both stress groups and the control group can be seen in Figure 9.1. Analyses of Variance (ANOVA) showed a main effect of group $[\mathrm{F}(2,44)=8.23 ; \mathrm{p}<.001]$ and time $[\mathrm{F}(5,220)=31.45 ; \mathrm{p}<.001]$, as well as the critical Group $\times$ Time interaction $[\mathrm{F}(10,220)=16.35 ; \mathrm{p}<.001]$. Thus, relative to the filler task in the control group, participants in both stress groups displayed significant increases in mean heart rate responses to TSST.

\footnotetext{
${ }^{34}$ Word lists were audio taped and played back on a digital voice recorder, thus ensuring that all participants heard the words at the same pace, tone of voice, volume, and intonation. All words were chosen from the Affective Norms for English Words list (ANEW; Bradley \& Lang, 1999) and were unanimously categorized as personality or memory words, respectively, in a pilot study $(N=10)$. Based on the ANEW normative ratings, memory and personality words did not differ with respect to mean valence, arousal, dominance, or word frequency (all $t$ s $<1$; all $p$ 's $>.43$ ).

${ }^{35}$ Due to technical failures, heart rate data from 5 individuals were lost.
} 


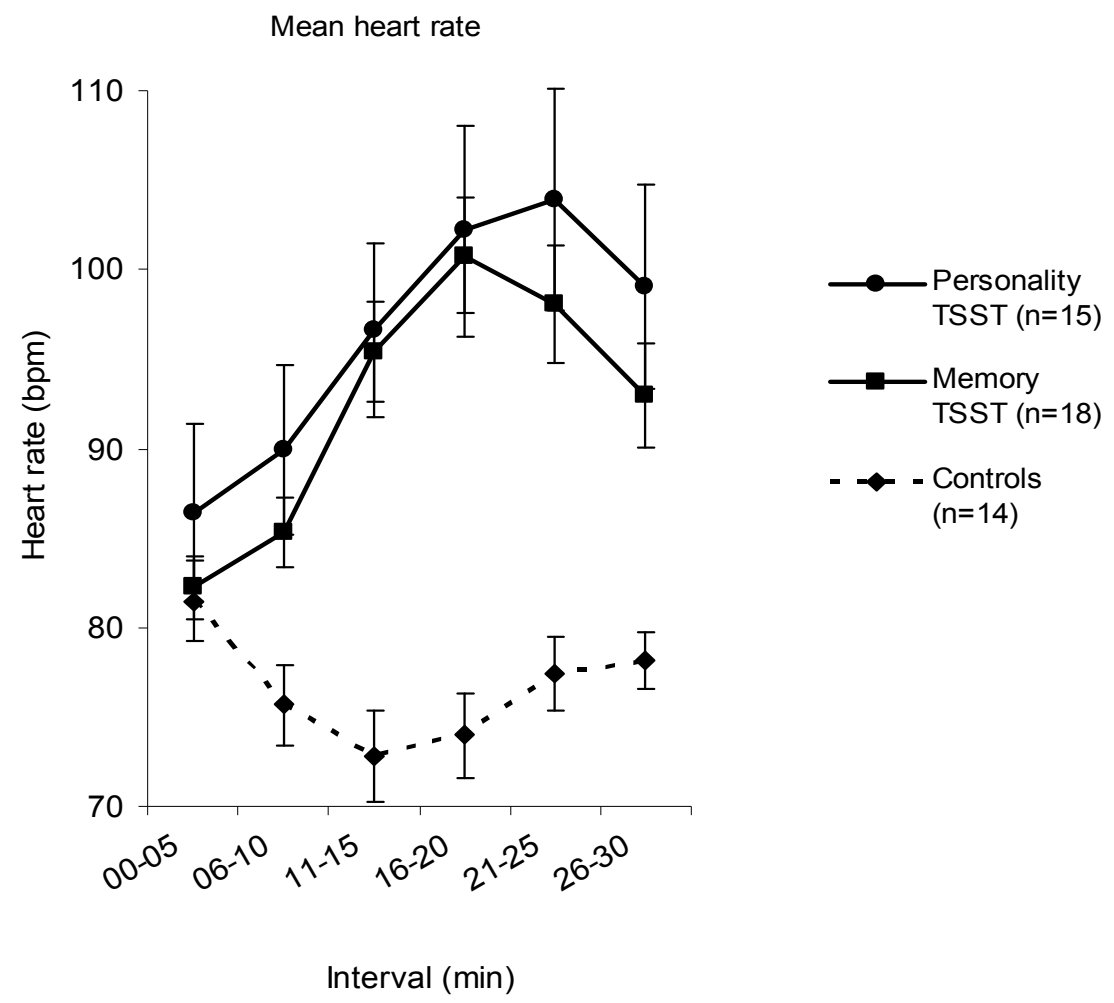

Figure 9.1. Mean heart rate responses expressed in beats per minute (bpm) for each of the 3 groups over time. Participants received stress or filler tasks in the 06-25 minute interval.

Delayed free recall performance was expressed as the percentage of words remembered in relation to the immediate free recall test (i.e., initially encoded words). Delayed free recall performance is shown in Figure 9.2. Repeated measures ANOVA yielded a significant main effect of group $[F(2,49)=4.45 ; p<.02]$, while no significant effect of word type (i.e., memory vs. personality words) was observed $[F(1$, $49)=2.49 ; p=.12]$. The critical Group $\times$ Word type showed a trend towards significance $[F(2,49)=2.76 ; p=.07]$. Follow-up $t$-tests indicated that relative to controls, personality-related stress resulted in improved delayed recall of personality words $(\phi<.05)$, but that memory-related stress did not enhance recall of memory words. 


\section{$24 \mathrm{~h}$ delayed recall}

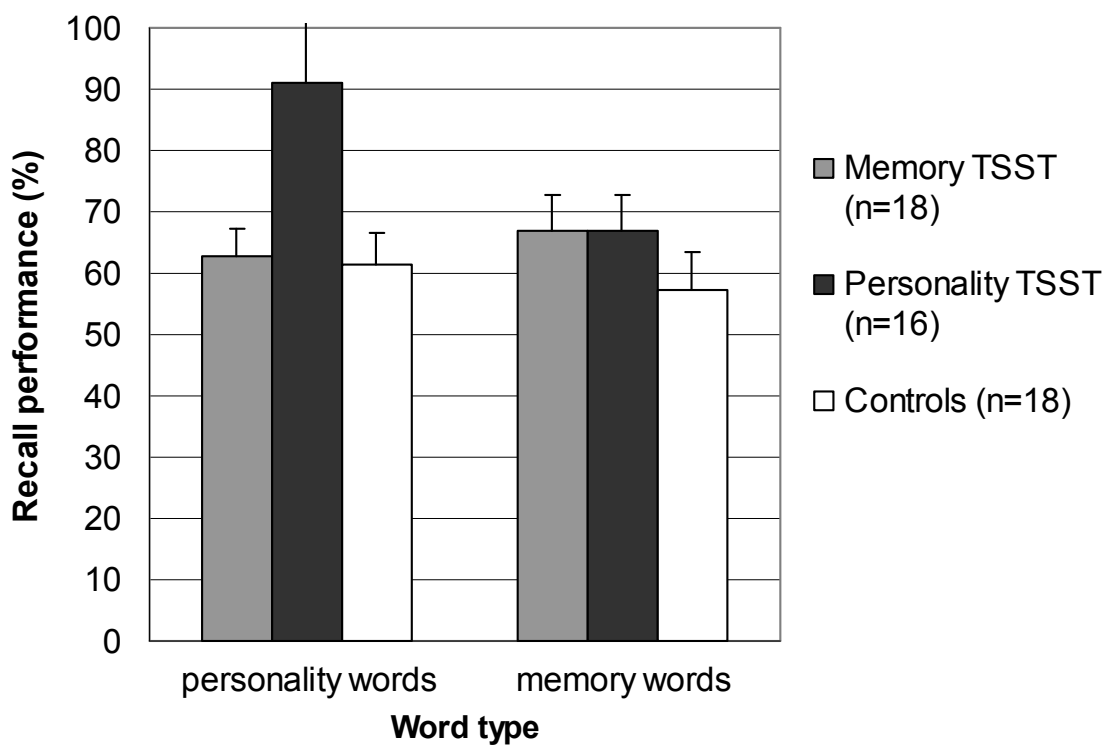

Figure 9.2. Mean scores on the delayed free recall test for the 3 groups.

Our pilot study thus provides some tentative evidence that exposure to a psychosocial challenge test may improve memory for context-related declarative memory tasks, though it should be noted that this effect was only observed for the personality-related stress group and that the crucial interaction term fell just short of being significant. One might intuitively think that this was because personality words showed more semantic cohesion than did memory words. However, the fact that there was no main effect of word type would argue against such an interpretation. Alternatively, one could hypothesize that this was due to a higher degree of personal relevance for the personality words, causing more self-reflection regarding their personality characteristics in participants during the 24 hour retention interval. We acknowledge, though, that these interpretations remain purely speculative. Future research should investigate the precise merits of the Joëls et al. (2006) framework and whether this model is, indeed, capable of accommodating for the various research findings in the stress and memory field. 


\section{State- and mood-state dependent effects}

As stated in the previous section, when the memory task is context-related to the stressor, memory performance is sometimes enhanced by stress exposure. However, this is not the only route through which context may affect remembering. A retrospective of context-dependent memory effects dates back to the work by Tulving and Thomson (1973). ${ }^{36}$ These authors proposed the idea, now known as the encoding specificity principle, that information is most accessible when the retrieval conditions match those at encoding. That is, one is more likely to remember a particular piece of information when the context of remembering closely resembles the context in which the information was encoded. ${ }^{37}$ Is it possible, then, that the impairing or enhancing effects of acute stress or cortisol elevations, are attributable to discrepancies in context?

In what is perhaps the most potent demonstration of the encoding specificity principle, Godden and Baddeley (1975) had scuba divers learn a list of words while either underwater or on land. Later on, they were tested for their recall of the words, again either underwater or on land. Godden and Baddeley found that participants who had learned the word list underwater recalled more words when they were tested in the same context (i.e., underwater) than when tested in a different context (i.e., on land). The reverse was true for those participants who had learned the words on land, with better recall when tested on land than when tested underwater. This implies that, indeed, context does matter such that when the encoding context is reinstated at retrieval, memory performance can be enhanced. On the other hand, follow-up studies (e.g., Saufley, Otaka, \& Bavaresco, 1985) have failed to replicate these context-dependent memory effects. This indicates that, even if there is an effect of context on memory performance, it probably remains rather small and flimsy.

Of course, Godden and Baddeley (1975) and Saufley and colleagues (1985) manipulated the physical, not the emotional retrieval context. Thus, one could speculate that mood during and shortly after stress exposure may be quite distinct from normal, everyday mood. Consequently, at a later retrieval test, moodcongruence and mood-incongruence effects may surface and obscure any memory effects that stress or the ensuing cortisol responses may have. This, however, seems rather unlikely. Building on earlier work (e.g., Bower, Monteiro, \& Gilligan, 1978), Bower (1981) asserted that recall of information is best when mood at retrieval is equivalent to mood at encoding (i.e., the mood-state-dependent-memory hypothesis). Though Bower et al. (1978) initially did find some preliminary evi-

\footnotetext{
36 Actually, the first experimental work dates back even further: Tulving and Osler (1968) and Thomson and Tulving (1970) provided the first demonstrations of what was later termed encoding specificity.

${ }^{37}$ Similar types of studies have also been published under a "transfer-appropriate processing" label. For examples, see Morris, Bransford, and Franks (1977) and Roediger, Weldon, and Challis (1989).
} 
dence to support the mood-state-dependent-memory hypothesis, subsequent work failed to find similar results (e.g., Bower \& Mayer, 1989; Foa, McNally, \& Murdock, 1989). Work by Eich (e.g., 1995) points in the same direction, suggesting that mood-state-dependent memory effects are fragile and only seem to occur when the mood experienced at encoding and retrieval is of high intensity.

Finally, more direct evidence against a context-dependent interpretation of findings demonstrating memory effects of acute stress and cortisol comes from a study by Wolf and co-workers (Wolf, Schommer, Hellhammer, Reischies, \& Kirschbaum, 2002). In this study, participants were required to learn a list of words either 1 hour before exposure to a TSST, or 10 minutes afterwards. Four weeks later, delayed recall performance was assessed, again either before or after being exposed to the TSST. Wolf et al. found that stress had no effect on initial learning or on delayed recall. More importantly, no evidence for an interaction between stress at initial learning and repeated stress at delayed recall was found. Though replication studies are needed to draw definitive conclusions, this suggests that context-dependent retrieval following stress exposure is a rather unlikely candidate to explain the effects of acute stress on declarative memory performance. ${ }^{38}$

\section{A glance at future research}

Overall, results from the present dissertation are not very encouraging for speculations about how acute stress may promote false recollections. There are, however, many avenues for future studies into the relationship between acute stress, cortisol elevations, and memory performance, as well as for studies investigating the antecedents and consequences of pseudo-memories. Below we will suggest some promising ideas for future studies.

Several factors have been found to modulate the associations between acute stress and memory performance. For example, both emotional valence and high arousal have been shown to be beneficial to memory performance following acute stress exposure or endogenous cortisol elevations (e.g., Abercrombie et al., 2006; Buchanan \& Lovallo, 2001; Jelicic, Geraerts, Merckelbach, \& Guerrieri, 2004; Kuhlmann \& Wolf, 2006b; Tops et al., 2003). Thus, an avenue for future research would be to further disentangle the contributions of stimulus valence and arousal in the relationship between acute stress and declarative memory performance. Of

\footnotetext{
${ }^{38}$ It is worth mentioning here that recent findings by Kuhlmann and Wolf (2006a) suggest that the environmental context per se may modulate the impairing effect of oral cortisol administration on declarative memory. That is, all else being equal, Kuhlmann and Wolf (2006a) reported that rendering retrieval conditions more relaxed (i.e., non-arousing) resulted in a nullification of the impairing effects of $30 \mathrm{mg}$ of cortisol on declarative memory performance, as compared to 2 reference studies (Kuhlmann, Kirschbaum, \& Wolf, 2005a; Kuhlmann \& Wolf, 2005) that used an identical methodology.
} 
course, there is evidence that the biological effects of emotionally positive and emotionally negative experiences show important similarities in that they are modulated by the amygdala (e.g., Hamann, Ely, Grafton, \& Kilts, 1999). This suggests that it is arousal that affects memory performance, independent of the valence of the memory material. Indeed, there have been studies highlighting the effect of arousal on recall performance. For example, studies by Bradley, Greenwald, Petry, and Lang (1992) and Bradley and Lang (2000) showed that pictures that were rated as either highly pleasant or unpleasant regarding their valence, were recalled more often than neutral pictures. Importantly, this effect was stronger for the highly arousing pictures. Moreover, arousal proved to be better at predicting memory performance than did valence (Bradley et al., 1992; Bradley \& Lang, 2000). In addition, Kuhlmann and Wolf (2006b) recently demonstrated that cortisol interacts with arousal in modulating memory performance.

Besides arousal, there are other factors that might account for the mnemonic advantage of emotional stimuli over neutral ones. Buchanan and co-workers, for example, have recently shown that semantic relatedness might add to the effects of high arousal on memory for emotional words (Buchanan, Etzel, Adolphs, \& Tranel, 2006). In delineating the exact "net" contributions of arousal and valence, cognitive neuroscience could play an important role as cognitive neuroscientists have only just begun to elucidate the neural mechanisms involved in emotional memory (e.g., Kensinger \& Corkin, 2004; Kensinger \& Schacter, 2006; for a recent review, see LaBar \& Cabeza, 2006).

Of late, there has been an increasing number of animal studies linking the prefrontal cortex (PFC) to the effects of stress on memory performance (e.g., Amat, Baratta, Paul, Bland, Watkins, \& Maier, 2005; Birnbaum et al., 2004; Shors, 2006). Of course, this issue is closely related to the neurobiological systems involved in the stress response (see also Chapter 1). That is to say, both the HPA axis and the noradrenergic system are vital regulatory mechanisms of the stress response, and may act on the hippocampus, amygdala, PFC, and various other brain areas (for reviews, see Arnsten, 1998; Charney, 2004; Elzinga \& Bremner, 2002). Recall that the noradrenergic system is responsible for the rapid responses to a stressor whereby adrenaline and noradrenaline are secreted, and that adrenaline seems to enhance the encoding of emotional memories by acting on the amygdala. In fact, several studies (e.g., Cahill \& McGaugh, 1998; Van Stegeren, Everaerd, Cahill, McGaugh, \& Gooren, 1998; but see Maheu, Joober, \& Lupien, $2005 \mathrm{~b})$ have identified a mediating effect of $\beta$-adrenergic activation of the amygdala complex in the memory enhancing effects of emotional arousal. Perhaps, then, the noradrenergic system may prove to be the most potent agent in the link between stress and declarative memory performance. As the research domain on GCs and memory is riveted by divergent results, it would be sensible to further investigate the precise role of the noradrenergic system and the PFC within this context. This could be done by investigating the effect of stress on certain brain 
regions by means of neuroimaging techniques, such as Event-Related Potentials (ERP's), Positron Emission Tomography (PET), or functional Magnetic Resonance Imaging (fMRI; e.g., Wang et al., 2005). Such reports may greatly contribute to our understanding of the precise nature of the effects of stress on memory performance.

As to research on pseudo-memories, Chapter 7 already advocated the idea that one should also look at the overt behavioural consequences of the applied memory manipulations. After all, the fundamental concern of people claiming to have recovered memories is that they are so persuaded of their memory accuracy, that these individuals will act on what they believe to be true (e.g., bringing the case to court or breaking off relations with the alleged perpetrator). In addition, when looking at real-life instances of recovered memories, one notices that - generally speaking - the individuals who experience them are people who are already, for reasons often unrelated to their recovered memories (e.g., an eating disorder, depression, family or marital problems), in psychotherapeutic treatment (e.g., Gudjonsson, 1997; Lief \& Fetkewicz, 1995; Merckelbach, 2003; van Koppen \& Merckelbach, 1999). It may well be that it are those individuals who suffer from psychopathology that are especially at risk for the development of pseudomemories. Future studies could, for example, benefit from looking at whether people suffering from major depressive disorder or other kinds of depression are particularly vulnerable to pseudo-memories.

Note that it is often assumed that memory impairments in depressed populations are caused by declines in cognitive resources (e.g., Estes, 1998; Hartlage, Alloy, Vazquez, \& Dykman, 1993). For example, Hertel and Milan (1994) found that relative to non-depressed controls, depressed individuals showed deficits in recalling words they had read during an earlier study session. Likewise, many studies have noted a link between overgeneralized autobiographical memories and depression. Thus, clinically depressed people are less specific on an autobiographical memory task in comparison to healthy controls (e.g., Van Vreeswijk \& De Wilde, 2004). It is also well-documented that people suffering from PTSD are characterized by various memory deficits (for reviews, see Isaac, Cushway, \& Jones, in press; McNally, 2003; Rosen, 2004). For example, people with PTSD show elevated levels of false recall and recognition in a standard DRM paradigm relative to controls (e.g., Bremner, Shobe, \& Kihlstrom, 2000; Zoellner, Foa, Brigidi, \& Przeworski, 2000). However, it is unclear whether depression, which is often associated with PTSD (Basoglu, Kilic, Salcioglu, \& Livanou, 2004; Shalev et al., 1998), modulates these effects. Indeed, Clancy, McNally, Schacter, Lenzenweger, and Pitman (2002) found a strong positive correlation between depressive symptoms and pseudomemories in a DRM paradigm. Additionally, Brennen and colleagues recently found that depression, and not degree of trauma exposure, predicted the number of false recollections in a DRM task among Bosnians with war-induced PTSD (Brennen, Dybdahl, \& Kapidzic, in press). Further support for a link between de- 
pression and false recollections as elicited by the DRM paradigm comes from a study of Moritz, Gläscher, and Brassen (2005). These authors asked a group of 25 patients that were diagnosed with either major depression or dysthymia and a group of 28 healthy controls to listen to a set of four DRM word lists. One list converged on a depression-relevant theme word (i.e., loneliness), one list was oriented around a delusion theme (i.e., betrayal) and another around a positive theme (i.e., holidays), while the fourth list converged on a neutral theme word (i.e., window). Moritz et al. (2005) found that relative to controls, depressed patients exhibited higher levels of false recognition, but only so for emotionally charged critical lures, particularly those that were depression-related. Focusing on clinical populations is important given that individual differences in pseudo-memory proneness among young healthy people are hard to detect and inconsistent at best (e.g., Heaps \& Nash, 1999; Horselenberg, 2005).

\section{Concluding remarks}

Summing up, the data of the present dissertation allow the following conclusions. In line with previous research, exposure to acute stress may negatively affect veridical memory performance. However, the pilot study described in Chapter 9 suggests that when acute stress is closely related in time to the encoding phase and is context-related to the to-be-remembered material, the effect of acute stress may reverse and, thus, might actually be beneficial to veridical memory performance. Similarly, this dissertation showed that acute stress may improve source monitoring performance. The main issue under investigation, however, was the hypothesis that acute stress and cortisol elevations may render people vulnerable to pseudomemories. This hypothesis remains unsubstantiated by the present results, as evidenced by the results from the studies described in Chapter 4. One may thus conclude that neither acute stress, nor cortisol responses enhance people's vulnerability to pseudo-memories. Future work should try to identify the precise antecedents of false recollections (e.g., by looking at clinical populations). The review of the pseudo-memory literature presented in the current dissertation, leads one to conclude that a fruitful avenue would be to focus on the behavioural consequences of such false beliefs or pseudo-memories.

In sum, should we conclude that stress is beneficial or detrimental to memory performance? Are stress and cortisol levels potential advocates of pseudomemories? It appears that there are no straightforward answers. That is, acute stress and cortisol may enhance memory formation under some circumstances, but generally tends to yield impairing effects on memory retrieval. With respect to the formation of pseudo-memories, results from earlier work and from the present dissertation have yielded mixed results, though the overall impression is that neither acute stress nor the ensuing cortisol elevations are responsible for the devel- 
opment of pseudo-memories. As the biological underpinnings of acute stress effects are complex, the diversity of research findings within the field is unlikely to be accounted for by a single mechanism or even just a few mechanisms. Clearly, the issues of who is more prone to developing false beliefs and/or pseudomemories, which circumstances may reduce or boost vulnerability to false recollections, and whether paradigms intended to investigate full-blown pseudo-memories are capable of eliciting behavioural consequences, are far from resolved. 


\section{Works cited}

Abercrombie, H. C., Kalin, N. H., Thurow, M. E., Rosenkranz, M. A., \& Davidson, R. J. (2003). Cortisol variation in humans affects memory for emotionally laden and neutral information. Behavioral Neuroscience, 117, 505-516.

Abercrombie, H. C., Speck, N. S., \& Monticelli, R. M. (2006). Endogenous cortisol elevations are related to memory facilitation only in individuals who are emotionally aroused. Psychoneuroendocrinology, 31, 187-196.

Ackil, J. K., \& Zaragoza, M. S. (1998). Memorial consequences of forced confabulation: Age differences in susceptibility to false memories. Developmental Psychology, 34, 1358-1372.

al' Absi, M., Hugdahl, K., \& Lovallo, W. R. (2002). Adrenocortical stress responses and altered working memory performance. Psychophysiology, 39, 95-99.

Alpert, J. L., Brown, L. S., \& Courtois, C. A. (1998). Symptomatic clients and memories of childhood sexual abuse: What the trauma and child sexual abuse literature tells us. Psychology, Public Policy, and Law, 4, 1052-1067.

Amat, J., Baratta, M. V., Paul, E., Bland, S. T., Watkins, L. R., \& Maier, S. F. (2005). Medial prefrontal cortex determines how behavioral control over stress impacts behavior and dorsal raphe nucleus activity. Nature Neuroscience, 8, 365371.

Amir, N., Freshman, M., Ramsey, B., Neary, E., \& Brigidi, B. (2001). Thoughtaction fusion in individuals with OCD symptoms. Behaviour Research and Therapy, 39, 765-776.

Andrews, B., Morton, J., Bekerian, D. A., Brewin, C. R., Davies, G. M., \& Mollon, P. (1995). The recovery of memories in clinical practice: Experiences and beliefs of British Psychological Society practitioners. The Psychologist, 8, 209-214.

Arnsten, A. F. T. (1998). Catecholamine modulation of prefrontal cognitive function. Trends in Cognitive Sciences, 2, 436-447.

Axelrod, J. (1974). Neurotransmitters. Scientific American, 230, 59-71.

Bartlett, F. C. (1932). Remembering: A study in experimental and social psychology. Oxford, England: Macmillan.

Basoglu, M., Kilic, C., Salciogl, E., \& Livanou, M. (2004). Prevalence of posttraumatic stress disorder and comorbid depression in earthquake survivors in Turkey: An epidemiological study. Journal of Traumatic Stress, 17, 133-141.

Beck, A. T., Steer, R. A., \& Garbin, M. G. (1988). Psychometric properties of the Beck Depression Inventory: Twenty-five years of evaluation. Clinical Psychology Review, 8, 77-100. 
Beck, A. T., Ward, C. H., Mendelson, M., Mock, J., \& Erbaugh, J. (1961). An inventory for measuring depression. Archives of General Psychiatry, 4, 561-571.

Beckwith, B. E., Petros, T. V., Scaglione, C., \& Nelson, J. (1986). Dose-dependent effects of hydrocortisone on memory in human males. Physiology and Behavior, 36, 283-286.

Benjamins, C., Asscheman, H., \& Schuurs, A. H. (1992). Increased salivary cortisol in severe dental anxiety. Psychophysiology, 29, 302-305.

Bergman, E. T., \& Roediger, H. L., III. (1999). Can Bartlett's repeated reproduction experiments be replicated? Memory \& Cognition, 27, 937-947.

Bernard, C. (1961). An introduction to the study of experimental medicine. New York: Collier. [Original work published as: Bernard, C. (1865). Introduction à l'étude de la médecine expérimentale. Paris: Éditions Garnier-Flammarion.]

Bernstein, D. M., Laney, C., Morris, E. K., \& Loftus, E. F. (2005a). False beliefs about fattening foods can have healthy consequences. Proceedings of the National Academy of Sciences of the United States of America, 102, 13724-13731.

Bernstein, D. M., Laney, C., Morris, E. K., \& Loftus, E. F. (2005b). False memories about food can lead to food avoidance. Social Cognition, 23, 11-34.

Bernstein, E. M., \& Putnam, F. W. (1986). Development, reliability, and validity of a dissociation scale. Journal of Nervous and Mental Disease, 174, 727-735.

Birnbaum, S. C., Yuan, P. X., Wang, M., Vijayraghavan, S., Bloom, A. K., Davis, D. J., et al. (2004). Protein kinase C overactivity impairs prefrontal cortical regulation of working memory. Science, 306, 882-884.

Blair, I. V., Lenton, A. P., \& Hastie, R. (2002). The reliability of the DRM paradigm as a measure of individual differences in false memories. Psychonomic Bulletin \& Review, 9, 590-596.

Boehning, D., \& Snyder, S. H. (2003). Novel neural modulators. Annual Review of Neuroscience, 26, 105-131.

Bower, G. H. (1981). Mood and memory. American Psychologist, 36, 129-148.

Bower, G. H., \& Mayer, J. D. (1989). In search of mood-dependent retrieval. Journal of Social Behavior and Personality, 4, 121-156.

Bower, G. H., Monteiro, K. P., \& Gilligan, S. G. (1978). Emotional mood as a context for learning and recall. Journal of Verbal Learning and Verbal Behavior, 17, 573-585.

Bradley, M. M., \& Lang, P. J. (1999). Affective norms for English words (ANEW): Instruction manual and affective ratings. Technical Report C-1, The Center for Research in Psychophysiology: University of Florida.

Bradley, M. M., \& Lang, P. J. (2000). Affective reactions to acoustic stimuli. Psychophysiology, 37, 204-215.

Bradley, M. M., Greenwald, M. K., Petry, M. C., \& Lang, P. J. (1992). Remembering pictures: Pleasure and arousal in memory. Journal of Experimental Psychology: Learning, Memory, and Cognition, 18, 379-390. 
Brainerd, C. J., \& Reyna, V. F. (2002). Fuzzy-trace theory and false memory. Current Directions in Psychological Science, 11, 164-169.

Brainerd, C. J., \& Reyna, V. F. (2004). Fuzzy-trace theory and memory development. Developmental Review, 24, 396-439.

Brébion, G., Smith, M. J., \& Widlocher, D. (1997). Discrimination and response bias in memory: Effects of depression severity and psychomotor retardation. Psychiatry Research, 70, 95-103.

Bremner, J. D. (1999). Does stress damage the brain? Biological Psychiatry, 45, $797-$ 805.

Bremner, J. D. (2002). Does stress damage the brain? Understanding trauma-related disorders from a mind-body perspective. New York: WW Norton.

Bremner, J. D. (2001). A biological model for delayed recall of childhood abuse. Journal of Aggression, Maltreatment and Trauma, 4, 165-183.

Bremner, J. D. (2003). Long-term effects of childhood sexual abuse on brain and neurobiology. Child and Adolescent Psychiatric Clinics, 12, 271-292.

Bremner, J. D., Krystal, J. H., Charney, D. S., \& Southwick, S. M. (1996). Neural mechanisms in dissociative amnesia for childhood abuse: Relevance to the current controversy surrounding the "false memory syndrome." American Journal of Psychiatry, 153(Suppl), 71-82.

Bremner, J. D., Shobe, K. K., \& Kihlstrom, J. F. (2000). False memories in women with self-reported childhood sexual abuse: An empirical study. Psychological Science, 11, 333-337.

Bremner, J. D., Vermetten, E., Afzal, N., \& Vythilingam, M. (2004). Deficits in verbal declarative memory function in women with childhood sexual abuserelated posttraumatic stress disorder. Journal of Nervous and Mental Disease, 192, 643-649.

Brennen, T., Dybdahl, R., \& Kapidzic, A. (in press). Trauma-related and neutral false memories in war-induced posttraumatic stress disorder. Consciousness and Cognition.

Brewin, C. R. (2003). Posttraumatic stress disorder: Malady or myth? New Haven, CT: Yale University Press.

Brown, M. W., \& Aggleton, J. P. (2001). Recognition memory: What are the roles of the perirhinal cortex and hippocampus? Nature Reviews Neuroscience, 2, 51-61.

Buchanan, T. W., \& Lovallo, W. R. (2001). Enhanced memory for emotional material following stress-level cortisol treatment in humans. Psychoneuroendocrinology, 26, 307-317.

Buchanan, T. W., Etzel, J. A., Adolphs, R., \& Tranel, D. (2006). The influence of autonomic arousal and semantic relatedness on memory for emotional words. International Journal of Psychophysiology, 61, 26-33.

Buckner, R. L., \& Wheeler, M. E. (2001). The cognitive neuroscience of remembering. Nature Reviews Neuroscience, 2, 624-634. 
Buske-Kirschbaum, A., von Auer, K., Krieger, S., Weis, S., Rauh, W., \& Hellhammer, D. (2003). Blunted cortisol responses to psychosocial stress in asthmatic children: A general feature of atopic disease? Psychosomatic Medicine, 65, 806-810.

Cabeza, R., Rao, S. M., Wagner, A. D., Mayer, A. R., \& Schacter, D. L. (2001). Can medial temporal lobe regions distinguish true from false? An event-related functional MRI study of veridical and illusory recognition memory. Proceedings of the National Academy of Sciences of the United States of America, 98, 4805-4810.

Cahill, L., \& McGaugh, J. L. (1998). Mechanisms of emotional arousal and lasting declarative memory. Trends in Neurosciences, 21, 294-299.

Candel, I. (2003). The truth and nothing but the truth: The accuracy, completeness, and consistency of emotional memories. Dissertation, Maastricht University, Maastricht.

Candel, I., Merckelbach, H., Loyen, S., \& Reyskens, H. (2005). "I hit the Shift-key and then the computer crashed": Children and false admissions. Personality and Individual Differences, 38, 1381-1387.

Cannon, W. B. (1929). Bodily changes in pain, hunger, fear, and rage. New York: Appleton.

Carrasco, G. A., \& Van de Kar, L. D. (2003). Neuroendocrine pharmacology of stress. European Journal of Pharmacology, 463, 235-272.

Cavenett, T., \& Nixon, R. D. V. (2006). The effect of arousal on memory for emotionally-relevant information: A study of skydivers. Behaviour Research and therapy, 44, 1461-1469.

Charney, D. S. (2004). Psychobiological mechanisms of resilience and vulnerability: Implications for successful adaptation to extreme stress. American Journal of Psychiatry, 161, 195-216.

Cima, M., Merckelbach, H., Nijman, H., Knauer, E., \& Hollnack, S. (2002). I can't remember your Honor: Offenders who claim amnesia. German Journal of Psychiatry, 5, 24-34.

Cima, M., Nijman, H., Merckelbach, H., Kremer, K., \& Hollnack, S. (2004). Claims of crime related amnesia in forensic patients. International Journal of Law and Psychiatry, 27, 215-221.

Clancy, S. A. (2005). Abducted: How people come to believe they were kidnapped by aliens. Cambridge, MA: Harvard University Press.

Clancy, S. A., McNally, R. J., Schacter, D. L. (1999). Effects of guided imagery on memory distortion in women reporting recovered memories of childhood sexual abuse. Journal of Traumatic Stress, 12, 559-569.

Clancy, S. A., McNally, R. J., Schacter, D. L., Lenzenweger, M. F., \& Pitman, R. K. (2002). Memory distortion in people reporting abduction by aliens. Journal of Abnormal Psychology, 111, 455-461.

Clancy, S. A., Schacter, D. L., McNally, R. J., \& Pitman, R. K. (2000). False recognition in women reporting recovered memories of sexual abuse. Psychological Science, 11, 26-31. 
Clow, A., Thorn, L., Evans, P., \& Hucklebridge, F. (2004). The awakening cortisol response: Methodological issues and significance. Stress, 7, 29-37.

Conrad, C. D., Jackson, J. L., Wieczorek, L., Baran, S. E., Harman, J. S., Wright, R. L., et al. (2004). Acute stress impairs spatial memory in male but not female rats: Influence of estrous cycle. Pharmacology, Biochemistry and Behavior, 78, 569579 .

Corwin, J. (1994). On measuring discrimination and response bias: Unequal numbers of targets and distractors and two classes of distractors. Neuropsychology, 8 , 110-117.

Crombag, H. F. M., Wagenaar, W. A., \& van Koppen, P. J. (1996). Crashing memories and the problem of 'source monitoring'. Applied Cognitive Psychology, 10, 95-104.

Crowne, D., \& Marlow, D. (1964). The approval motive. New York: Wiley.

Curran, T., Schacter, D. L., Johnson, M. K., \& Spinks, R. (2001). Brain potentials reflect behavioral differences in true and false recognition. Journal of Cognitive Neuroscience, 13, 201-216.

Davis, D., \& Loftus, E. F. (in press). Internal and external sources of distortion in adult witness memory. In M. P. Toglia, J. D. Read, D. R. Ross, R. C. L. Lindsay, Handbook of eyewitness memory (Vol. 1): Memory for events. Mahwah, NJ: Erlbaum.

Davis, D., Loftus, E. F., \& Follette, W. C. (2001). Commentary on "Standards for informed consent in recovered memory therapy": How, when, and whether to use informed consent for recovered memory therapy. Journal of the American Academy of Psychiatry and the Law, 29, 148-159.

de Groot, M. H. (1991). Psychometrische aspecten van een stemmingsschaal (verkorte POMS). [Psychometric properties of a mood scale (shortened POMS)] Gedrag en Gezondheid [Behaviour and Health], 20, 46-51.

de Kloet, E. R., Oitzl, M. S., \& Joëls, M. (1999). Stress and cognition: Are corticosteroids good or bad guys? Trends in Neurosciences, 22, 422-426.

de Quervain, D. J. F., Roozendaal, B., Nitsch, R. M., McGaugh, J. L., \& Hock, C. (2000). Acute cortisone administration impairs retrieval of long-term declarative memory in humans. Nature Neuroscience, 3, 313-314.

de Quervain, D. J. F., Henke, K., Aerni, A., Treyer, V., McGaugh, J. L., Berthold, T., et al. (2003). Glucocorticoid-induced impairment of declarative memory retrieval is associated with reduced blood flow in the medial temporal lobe. European Journal of Neuroscience, 17, 1296-1302.

Deelman, B. G., Brouwer, W. H., van Zomeren, A. H., \& Saan, R. J. (1980). Functiestoornissen na trauma capitis. [Deficiencies in functioning following trauma capitis] In A. Jennekens-Schinkel (Ed.), Neuropsychologie in Nederland [Neuropsychology in the Netherlands]. Deventer: Van Loghum Slaterus.

Deese, J. (1959). On the prediction of occurrence of particular verbal intrusions in immediate recall. Journal of Experimental Psychology, 58, 17-22. 
Dickerson, S. S., \& Kemeny, M. E. (2004). Acute stressors and cortisol responses: A theoretical integration and synthesis of laboratory research. Psychological Bulletin, 130, 355-391.

Dimond, S. J. (1980). Neuropsychology. A textbook of systems and psychological functions of the human brain. London: Butterworth.

Dobbins, I. G., Foley, H., Schacter, D. L., \& Wagner, A. D. (2002). Executive control during episodic retrieval: Multiple prefrontal processes subserve source memory. Neuron, 35, 989-996.

Dobbins, I. G., Rice, H. J., Wagner, A. D., \& Schacter, D. L. (2003). Memory orientation and success: Separable neurocognitive components underlying episodic recognition. Neuropsychologia, 41, 318-333.

Dodhia, R. M., \& Metcalfe, J. (1999). False memories and source monitoring. Cognitive Neuropsychology, 16, 489-508.

Domes, G., Heinrichs, M., Reichwald, U., \& Hautzinger, M. (2002). Hypothalamicpituitary-adrenal axis reactivity to psychological stress and memory in middleaged women: High responders exhibit enhanced declarative memory performance. Psychoneuroendocrinology, 27, 843-853.

Domes, G., Heinrichs, M., Rimmele, U., Reichwald, U., \& Hautzinger, M. (2004). Acute stress impairs recognition for positive words: Association with stressinduced cortisol secretion. Stress, 7, 173-181.

Domes, G., Rothfischer, J., Reichwald, U., \& Hautzinger, M. (2005). Inverted-U function between salivary cortisol and retrieval of verbal memory after hydrocortisone treatment. Behavioral Neuroscience, 119, 512-517.

Eich, E. (1995). Searching for mood dependent memory. Psychological Science, 6, $67-$ 75.

Eisen, M. L., \& Carlson, E. B. (1998). Individual differences in suggestibility: Examining the influence of dissociation, absorption, and a history of childhood abuse. Applied Cognitive Psychology, 12, S47-S61.

Elzinga, B. M., Bakker, A., \& Bremner, J. D. (2005). Stress-induced cortisol elevations are associated with impaired delayed, but not immediate recall. Psychiatry Research, 134, 211-223.

Elzinga, B. M., \& Bremner, J. D. (2002). Are the neural substrates of memory the final common pathway in posttraumatic stress disorder (PTSD)? Journal of Affective Disorders, 70, 1-17.

Elzinga, B. M., \& Roelofs, K. (2005). Cortisol-induced impairments of working memory require acute sympathetic activation. Behavioral Neuroscience, 119, 98103.

Estes, W. K. (1998). Models of human memory and their implications for research on aging and psychopathology. Development and Psychopathology, 10, 607-624.

Fabiani, M., Stadler, M. A., \& Wessels, P. M. (2000). True but not false memories produce a sensory signature in human lateralized brain potentials. Journal of Cognitive Neuroscience, 12, 941-949. 
Field, A. P., \& Lawson, J. (2003). Fear information and the development of fears during childhood: Effects on implicit fear responses and behavioural avoidance. Behaviour Research and Therapy, 41, 1277-1293.

Field, A. P., Argyris, N. G., Knowles, K. A. (2001). Who's afraid of the big bad wolf: A prospective paradigm to test Rachman's indirect pathways in children. Behaviour Research and Therapy, 39, 1259-1276.

Fiske, S. T. (1992). Thinking is for doing: Portraits of social cognition from Daguerreotype to laserphoto. Journal of Personality and Social Psychology, 63, 877-889.

Foa, E. B., McNally, R. J., \& Murdock, T. B. (1989). Anxious mood and memory. Behaviour Research and Therapy, 27, 141-147.

Foa, E. B., Riggs, D. S., Dancu, C. V., \& Rothbaum, B. O. (1993). Reliability and validity of a brief instrument for assessing post-traumatic stress disorder. Journal of Traumatic Stress, 6, 459-473.

Frankenhaeuser, M., Dunne, E., \& Lundberg, U. (1976). Sex differences in sympathetic-adrenal medullary reactions induced by different stressors. Psychopharmacology, 47, 1-5.

Freyd, J. J., \& Gleaves, D. H. (1996). "Remembering" words not presented in lists: Relevance to the current recovered/false memory controversy. Journal of Experimental Psychology: Learning, Memory, and Cognition, 22, 811-813.

Gaab, J., Rohleder, N., Nater, U. M., \& Ehlert, U. (2005). Psychological determinants of the cortisol stress response: The role of anticipatory cognitive appraisal. Psychoneuroendocrinology, 30, 599-610.

Gardiner, J. M., \& Java, R. I. (1993). Recognising and remembering. In A. F. Collins \& S. E. Gathercole (Eds.), Theories of memory (pp. 163-188). Hillsdale, NJ: Lawrence Erlbaum.

Gardiner, J. M., Ramponi, C., \& Richardson Klavehn, A. (2002). Recognition memory and decision processes: A meta-analysis of remember, know, and guess responses. Memory, 10, 83-98.

Garry, M., \& Polascheck, D. L. L. (2000). Imagination and memory. Current Directions in Psychological Science, 9, 6-10.

Garry, M., Manning, C. G., Loftus, E. F., \& Sherman, S. J. (1996). Imagination inflation: Imagining a childhood event inflates confidence that it occurred. Psychonomic Bulletin \& Review, 3, 208-214.

Geraerts, E. (2006). Remembrance of things past: The cognitive psychology of remembering and forgetting trauma. Dissertation, Maastricht University, Maastricht.

Geraerts, E., Arnold, M. M., Lindsay, D. S., Merckelbach, H., Jelicic, M., \& Hauer, B. (in press). Forgetting of prior remembering in persons reporting recovered memories of childhood sexual abuse. Psychological Science.

Geraerts, E., Smeets, E., Jelicic, M., Merckelbach, H., \& van Heerden, J. (2006). Retrieval inhibition of trauma-related words in women reporting repressed or recovered memories of childhood sexual abuse. Behaviour Research and Therapy, 44, 1129-1136. 
Geraerts, E., Smeets, E., Jelicic, M., van Heerden, J., \& Merckelbach, H. (2005). Fantasy proneness, but not self-reported trauma is related to DRM performance of women reporting recovered memories of childhood sexual abuse. Consciousness and Cognition, 14, 602-612.

Giesbrecht, T. (2006). Dissociation: A defensive manewver? Dissertation, Maastricht University, Maastricht.

Giesbrecht, T., Smeets, T., Merckelbach, H., \& Jelicic, M. (in press). Depersonalization experiences in undergraduates are related to heightened stress cortisol responses. The Journal of Nervous and Mental Disease.

Godden, D. R., \& Baddeley, A. D. (1975). Context-dependent memory in two natural environments: On land and underwater. British Journal of Psychology, 66, 325-331.

Goff, L. M., \& Roediger, H. L., III. (1998). Imagination inflation for action events: Repeated imaginings lead to illusory recollections. Memory \& Cognition, 26, 2033.

Gomperts, W. (1996). Herinneringen aan het hiervoormaals. [Memories of past lives] Maandblad Geestelijke Volksgezondheid [Monthly Journal of Public Mental Health7, 7/8, 777-800.

Granhag, P. A., Strömwall, L., \& Billings, J. (2003). I'll never forget the sinking ferry: How social influence makes false memories surface. In M. Vanderhallen, G. Vervaeke, P. J. van Koppen, \& J. Goethals (Eds.), Much ado about crime: Chapters on psychology and law (pp. 129-140). Brussels: Politeia.

Grice, H. P. (1989). Studies in the way of words. London: Harvard University Press.

Gudjonsson, G. H. (1989). Compliance in an interrogative situation: A new scale. Personality and Individual Differences, 10, 535-540.

Gudjonsson, G. H. (1997). Accusations by adults of childhood sexual abuse: A survey of the members of the British False Memory Society (BFMS). Applied Cognitive Psychology, 11, 3-18.

Gudjonsson, G. H., \& Clare, I. C. H. (1995). The relationship between confabulation and intellectual ability, memory, interrogative suggestibility and acquiescence. Personality and Individual Differences, 19, 333-338.

Gudjonsson, G. H., \& MacKeith, J. A. C. (1982). False confessions: Psychological effects of interrogation. A discussion paper. In A. Trankell (Ed.), Reconstructing the past: The role of psychologists in criminal trials (pp. 253-269). Deventer: Kluwer.

Gunnar, M. R., Morison, S. J., Chisholm, K., \& Schuder, M. (2001). Salivary cortisol levels in children adopted from Romanian orphanages. Development and Psychopathology, 13, 611-628.

Haber, R. N., \& Haber, L. (2000). Experiencing, remembering and reporting events. Psychology, Public Policy, and Law, 6, 1057-1097.

Hamann, S. B., Ely, T. D., Grafton, S. T., \& Kilts, C. D. (1999). Amygdala activity related to enhanced memory for pleasant and aversive stimuli. Nature Neuroscience, 2, 289-293. 
Hartlage, S., Alloy, L. B., Vazquez, C. V., \& Dykman, B. M. (1993). Automatic and effortful processing in depression. Psychological Bulletin, 113, 247-278.

Health Council of the Netherlands. (2004). Disputed memories. The Hague: Health Council of the Netherlands; Publication no. 2004/02.

Heaps, C., \& Nash, M. (1999). Individual differences in imagination inflation. Psychonomic Bulletin \& Review, 6, 313-318.

Henkel, L. A., Coffman, K. J. (2004). Memory distortions in coerced false confessions: A source monitoring framework analysis. Applied Cognitive Psychology, 18, 567-588.

Henquet, C., Krabbendam, L., Dautzenberg, J., Jolles, J., \& Merckelbach, H. (2005). Confusing thoughts and speech: Source monitoring and psychosis. Psychiatry Research, 133, 57-63.

Hermans, D., \& De Houwer, J. (1994). Affective and subjective familiarity ratings of 740 Dutch words. Psychologica Belgica, 34, 115-139.

Hertel, P. T., \& Milan, S. (1994). Depressive deficits in recognition: Dissociation of recollection and familiarity. Journal of Abnormal Psychology, 103, 736-742.

Het, S., Ramlow, G., \& Wolf, O. T. (2005). A meta-analytic review of the effects of acute cortisol administration on human memory. Psychoneuroendocrinology, 30, 771-784.

Hoffman, R., \& al' Absi, M. (2004). The effect of acute stress on subsequent neuropsychological test performance (2003). Archives of Clinical Neuropsychology, 19, 497-506.

Horselenberg, R. (2005). Pseudo-memories and personality characteristics. Dissertation, Maastricht University, Maastricht.

Horselenberg, R., \& Merckelbach, H. (submitted for publication). Fantasy proneness and dissociative experiences in adults with previous-life memories.

Horselenberg, R., Merckelbach, H., \& Josephs, S. (2003). Individual differences and false confessions: A conceptual replication of Kassin and Kiechel (1996). Psychology, Crime \& Law, 9, 1-8.

Horselenberg, R., Merckelbach, H., Muris, P., Rassin, E., Sijsenaar, M., \& Spaan, V. (2000). Imagining fictitious childhood events: The role of individual differences in imagination inflation. Clinical Psychology and Psychotherapy, 7, 128-137.

Horselenberg, R., Merckelbach, H., Smeets, T., Franssens, D., Peters, G. J. Y. \& Zeles, G. (2006). False confessions in the lab: Do plausibility and consequences matter? Psychology, Crime \& Law, 12, 61-75.

Howe, M. L. (1998). Individual differences in factors that modulate storage and retrieval of traumatic memories. Development and Psychopathology, 10, 681-698.

Hyman, I. E. J., \& Billings, F. J. (1998). Individual differences and the creation of false childhood memories. Memory, 6, 1-20.

Hyman, I. E. J., \& Kleinknecht, E. E. (1999). False childhood memories: Research, theory, and applications. In L. M. Williams \& V. L. Banyard (Eds.), Trauma and memory (pp. 175-188). Thousand Oaks, CA: Sage Publications, Inc. 
Hyman, I. E. J., \& Pentland, J. (1996). The role of mental imagery in the creation of false childhood memories. Journal of Memory and Language, 35, 101-117.

Hyman, I. E. J., Husband, T. H., \& Billings, F. J. (1995). False memories of childhood experiences. Applied Cognitive Psychology, 9, 181-197

Hyman, I. E., J., \& Loftus, E. F. (1998). Errors in autobiographical memory. Clinical Psychology Review, 18, 933-947.

Isaac, C. L., Cushway, D., \& Jones, G. V. (in press). Is posttraumatic stress disorder associated with specific deficits in episodic memory? Clinical Psychology Review.

Janowsky, J. S., Shimamura, A. P., \& Squire, L. R. (1989). Source memory impairment in patients with frontal lobe lesions. Neuropsychologia, 27, 1043-1056.

Jelicic, M., \& Bonke, B. (2001). Memory impairments following chronic stress? A critical review. European Journal of Psychiatry, 15, 225-232.

Jelicic, M., Geraerts, E., Merckelbach, H., \& Guerrieri, R. (2004). Acute stress enhances memory for emotional words, but impairs memory for neutral words. International Journal of Neuroscience, 114, 1343-1351.

Jelicic, M., \& Merckelbach, H. (2004). Traumatic stress, brain changes, and memory deficits: A critical note. Journal of Nervous and Mental Disease, 192, 548-553.

Jelicic, M., Smeets, T., Peters, M. J. V., Candel, I., Horselenberg, R., \& Merckelbach, H. (2006). Assassination of a controversial politician: Remembering details from another non-existent film. Applied Cognitive Psychology, 20, 591-596.

Joëls, M., Pu, Z., Wiegert, O., Oitzl, M. S., \& Krugers, H. J. (2006). Learning under stress: How does it work? Trends in Cognitive Sciences, 10, 152-158.

Johnson, M. K., \& Raye, C. L. (1998). False memories and confabulation. Trends in Cognitive Sciences, 2, 137-145.

Johnson, M. K., \& Raye, C. L. (2000). Cognitive and brain mechanisms of false memories and beliefs. In D. L. Schacter \& E. Scarry (Eds.), Memory, brain, and belief (pp. 35-86). Cambridge, MA: Harvard University Press.

Johnson, M. K., Foley, M. A., Suengas, A. G., \& Raye, C. L. (1988). Phenomenal characteristics of memories for perceived and imagined autobiographical events. Journal of Experimental Psychology: General, 117, 371-376.

Johnson, M. K., Hashtroudi, S., \& Lindsay, D. S. (1993). Source monitoring. Psychological Bulletin, 114, 3-28.

Joseph, R. (1999). The neurology of traumatic dissociative amnesia: Commentary and literature review. Child Abuse \& Neglect, 23, 715-727.

Kajantie, E., \& Phillips, D. I. W. (2006). The effects of sex and hormonal status on the physiological response to acute psychosocial stress. Psychoneuroendocrinology, 31, 151-178.

Kensinger, E. A., \& Corkin, S. (2004). Two routes to emotional memory: Distinct neural processes for valence and arousal. Proceedings of the National Academy of Sciences of the United States of America, 101, 3310-3315. 
Kensinger, E. A., \& Schacter, D. L. (2006). Processing emotional pictures and words: Effects of valence and arousal. Cognitive, Affective, \& Behavioral Neuroscience, 6, 110-126.

Kassin, S. M., \& Kiechel, K. L. (1996). The social psychology of false confessions: Compliance, internalization, and confabulation. Psychological Science, 7, 125-128.

Kihlstrom, J. F. (2006). Trauma and memory revisited. In: B. Uttl , N. Ohta, \& A. L. Siegenthaler (Eds.), Memory and emotions: Interdisciplinary perspectives (pp. 259292) New York: Blackwell.

Kirschbaum, C., \& Hellhammer, D. H. (1989). Salivary cortisol in psychobiological research: An overview. Neuropsychobiology, 22, 150-169.

Kirschbaum, C., \& Hellhammer, D. H. (1994). Salivary cortisol in psychoneuroendocrine research: Recent developments and applications. Psychoneuroendocrinology, 19, 313-333.

Kirschbaum, C., Kudielka, B. M., Gaab, J., Schommer, N. C., \& Hellhammer, D. H. (1999). Impact of gender, menstrual cycle phase, and oral contraceptives on the activity of the hypothalamic-pituitary-adrenal axis. Psychosomatic Medicine, 61, 154-162.

Kirschbaum, C., Pirke, K.-M., \& Hellhammer, D. H. (1993). The 'Trier Social Stress Test': A tool for investigating psychobiological stress responses in a laboratory setting. Neuropsychobiology, 28, 76-81.

Kirschbaum, C., Schommer, N., Federenko, I., Gaab, J., Neumann, O., Oellers, M., et al. (1996a). Short-term estradiol treatment enhances pituitary-adrenal axis and sympathetic responses to psychosocial stress in healthy young men. Journal of Clinical Endocrinology and Metabolism, 81, 3639-3643.

Kirschbaum, C., Wolf, O. T., May, M., Wippich, W., \& Hellhammer, D. H. (1996b). Stress- and treatment-induced elevations of cortisol levels associated with impaired declarative memory in healthy adults. Life Sciences, 58, 1475-1483.

Kirschbaum, C., Wüst, S., \& Hellhammer, D. (1992). Consistent sex differences in cortisol responses to psychological stress. Psychosomatic Medicine, 54, 648-657.

Koehler, D. J. (1991). Explanation, imagination, and confidence in judgment. Psychological Bulletin, 110, 499-519.

Kopelman, M. D. (1995). The assessment of psychogenic amnesia. In: A. D. Baddeley, B.A. Wilson, \& F. N. Watts (Eds.), Handbook of Memory Disorders (pp. 427-448). New York: John Wiley.

Kopelman, M. D. (1999). Varieties of false memory. Cognitive Neuropsychology, 16, 197-214.

Koriat, A., \& Goldsmith, M. (1994). Memory in naturalistic and laboratory contexts: Distinguishing the accuracy-oriented and quantity-oriented approaches to memory assessment. Journal of Experimental Psychology: General, 123, 297-315.

Koriat, A., \& Goldsmith, M. (1996). Monitoring and control processes in the strategic regulation of memory accuracy. Psychological Review, 103, 490-517. 
Koriat, A., Goldsmith, M., \& Pansky, A. (2000). Toward a psychology of memory accuracy. Annual Review of Psychology, 51, 481-537.

Kudielka, B. M., Buske-Kirschbaum, A., Hellhammer, D. H., \& Kirschbaum, C. (2004a). HPA axis responses to laboratory psychosocial stress in healthy adults, younger adults, and children: Impact of age and gender. Psychoneuroendocrinology, 29, 83-98.

Kudielka, B. M., Hellhammer, J., Hellhammer, D. H., Wolf, O. T., Pirke, K. M., Varadi, E., et al. (1998). Sex differences in endocrine and psychological responses to psychosocial stress in healthy elderly subjects and the impact of a 2 week dehydroepiandrosterone treatment. Journal of Clinical Endocrinology and $\mathrm{Me}$ tabolism, 83, 1756-1761.

Kudielka, B. M., \& Kirschbaum, C. (2003). Awakening cortisol responses are influenced by health status and awakening time but not by menstrual cycle phase. Psychoneuroendocrinology, 28, 35-47.

Kudielka, B. M., \& Kirschbaum, C. (2005). Sex differences in HPA axis responses to stress: A review. Biological Psychology, 69, 113-132.

Kudielka, B. M., Schommer, N. C., Hellhammer, D. H., \& Kirschbaum, C. (2004b). Acute HPA axis responses, heart rate, and mood changes to psychosocial stress (TSST) in humans at different times of day. Psychoneuroendocrinology, 29, 983-992.

Kuhlmann, S., Kirschbaum, C., \& Wolf, O. T. (2005a). Effects of oral cortisol treatment in healthy young women on memory retrieval of negative and neutral words. Neurobiology of Learning and Memory, 83, 158-162.

Kuhlmann, S., Piel, M., \& Wolf, O. T. (2005b). Impaired memory retrieval after psychosocial stress in healthy young men. Journal of Neuroscience, 25, 2977-2982.

Kuhlmann, S., \& Wolf, O. T. (2005). Cortisol and memory retrieval in women: Influence of menstrual cycle and oral contraceptives. Psychopharmacology, 183, 65-71.

Kuhlmann, S., \& Wolf, O. T. (2006a). A non-arousing test situation abolishes the impairing effects of cortisol on delayed memory retrieval in healthy women. Neuroscience Letters, 399, 268-272.

Kuhlmann, S., \& Wolf, O. T. (2006b). Arousal and cortisol interact in modulating memory consolidation in healthy young men. Behavioral Neuroscience, 120, $217-$ 223.

Kunz-Ebrecht, S. R., Kirschbaum, C., Marmot, M., \& Steptoe, A. (2004). Differences in cortisol awakening response on work days and weekends in women and men from the Whitehall II cohort. Psychoneuroendocrinology, 29, 516-528.

LaBar, K. S., \& Cabeza, R. (2006). Cognitive neuroscience of emotional memory. Nature Reviews Neuroscience, 7, 54-64.

Ladouceur, R., Rheaume, J., Freeston, M. H., Aublet, F., Jean, K., Lachance, S., Langlois, F., de Pokomandy-Morin, K. (1995). Experimental manipulations of 
responsibility: An analogue test for models of obsessive-compulsive disorder. Behaviour Research and Therapy, 33, 937-946.

Lampinen, J. M., Neuschatz, J. S., \& Payne, D. G. (1998). Memory illusions and consciousness: Examining the phenomenology of true and false memories. Current Psychology: Developmental, Learning, Personality, Social, 16, 181-224.

Larson, M. C., Gunnar, M. R., \& Hertsgaard, L. (1991). The effects of morning naps, car trips, and maternal separation on adrenocortical activity in human infants. Child Development, 62, 362-372.

Lewis, M., \& Thomas, D. (1990). Cortisol release in infants in response to inoculation. Child Development, 61, 50-59.

Lezak, M. D. (1995). Profile of Mood States (POMS). In M. D. Lezak (Ed.), Neuropsychological assessment (3 ${ }^{\text {rd }}$ ed.) (pp. 786-787). London: Oxford University Press.

Lezak, M. D. (2004a). Auditory-Verbal Learning Test (AVLT). In M. D. Lezak, D. B. Howieson, \& D. W. Loring (Eds.), Neuropsychological assessment (4th ed.) (pp. 422-429; 686; 687; 696; 724). London: Oxford University Press.

Lezak, M. D. (2004b). Digit span. In M. D. Lezak, D. B. Howieson, \& D. W. Loring (Eds.), Neuropsychological assessment (4th ed.) (pp. 351-353; 360; 649-650; 652; 656; 658-659; 760-761; 765). London: Oxford University Press.

Lezak, M. D. (2004c). Profile of Mood States (POMS). In M. D. Lezak, D. B. Howieson, \& D. W. Loring (Eds.), Neuropsychological assessment (4th ed.) (pp. 751752). London: Oxford University Press.

Lieberman, H. R., Bathalon, G. P., Falco, C. M., Kramer, F. M., Morgan, C. A., III, \& Niro, P. (2005). Severe decrements in cognition function and mood induced by sleep loss, heat, dehydration, and undernutrition during simulated combat. Biological Psychiatry, 57, 422-429.

Lief, H. I., \& Fetkewicz, J. (1995). Retractors of false memories: The evolution of pseudomemories. Journal of Psychiatry and Law, 23, 411-435.

Lindsay, D. S. (1990). Misleading suggestions can impair eyewitnesses' ability to remember event details. Journal of Experimental Psychology: Learning, Memory, and Cognition, 16, 1077-1083.

Lindsay, D. S. (1994). Memory source monitoring and eyewitness testimony. In D. F. Ross \& J. D. Read (Eds.), Adult eyewitness testimony: Current trends and developments (pp. 27-55). New York: Cambridge University Press.

Lindsay, D. S. (1997). Increasing sensitivity. In J. D. Read \& D. S. Lindsay (Eds.), Recollections of trauma: Scientific evidence and clinical practice (pp. 1-16). New York: Plenum Press.

Loftus, E. F. (2001). Imagining the past. The Psychologist, 14, 584-587.

Loftus, E. F. (2003). Make-believe memories. American Psychologist, 58, 867-873.

Loftus, E. F. (2004). Dispatch from the (un)civil memory wars. Lancet, 364, S20S21.

Loftus, E. F. (2005). Planting misinformation in the human mind: A 30-year investigation of the malleability of memory. Learning and Memory, 12, 361-366. 
Loftus, E. F., \& Bernstein, D. M. (2005). Rich false memories: The royal road to success. In A. F. Healy (Ed.), Experimental cognitive psychology and its applications (pp. 101-113). Washington DC: American Psychological Association Press.

Loftus, E. F., \& Davis, D. (2006). Recovered memories. Annual Review of Clinical Psychology, 2, 469-498.

Loftus, E. F., \& Ketcham, K. (1994). The myth of repressed memory: False memories and allegations of sexual abuse. New York: St. Martin's Press.

Loftus, E. F., \& Mazzoni, G. A. L. (1998). Using imagination and personalized suggestion to change people. Behavior Therapy, 29, 691-706.

Loftus, E. F., \& Palmer, J. C. (1974). Reconstruction of automobile destruction: An example of the interaction between language and memory. Journal of Verbal Learning and Verbal Behavior, 13, 585-589.

Loftus, E. F., \& Pickrell, J. E. (1995). The formation of false memories. Psychiatric Annals, 25, 720-725.

Loftus, E. F., \& Zanni, G. (1975). Eyewitness testimony: The influence of the wording of a question. Bulletin of the Psychonomic Society, 5, 86-88.

Lovallo, W. R. (2005). Stress \& health: Biological and psychological interactions (2nd ed.). Thousand Oaks, CA: Sage Publications.

Lovallo, W. R., \& Thomas, T. L. (2000). Stress hormones in psychophysiological research: Emotional, behavioral, and cognitive implications. In J. T. Cacioppo \& L. G. Tassinary (Eds.), Handbook of psychophysiology (2nd ed.) (pp. 342-367). New York: Cambridge University Press.

Luine, V. N. (2002). Sex differences in chronic stress effects on memory in rats. Stress, 5, 205-216.

Lupien, S. J., Fiocco, A., Wan, N., Maheu, F., Lord, C., Schramek, T., et al. (2005). Stress hormones and human memory function across the lifespan. Psychoneuroendocrinology, 30, 225-242.

Lupien, S. J., \& Lepage, M. (2001). Stress, memory, and the hippocampus: You can't live with it, can't live without it. Behavioral Brain Research, 127, 137-158.

Lupien, S. J., \& McEwen, B. S. (1997). The acute effects of corticosteroids on cognition: Integration of animal and human model studies. Brain Research Reviens, 24, 1-27.

Lupien, S. J., Gaudreau, S., Tchiteya, B. M., Maheu, F., Sharma, S., Nair, N. P. V., Hauger, R. L., McEwen, B. S., \& Meaney, M. J. (1997). Stress-induced declarative memory impairment in healthy elderly subjects: Relationship to cortisol reactivity. Journal of Clinical Endocrinology and Metabolism, 82, 2070-2075.

Lupien, S. J., Gillin, C. J., \& Hauger, R. L. (1999). Working memory is more sensitive than declarative memory to the acute effects of corticosteroids: A doseresponse study in humans. Behavioral Neuroscience, 113, 420-430.

Lupien, S. J., Wilkinson, C. W., Briere, S., Ng Ying Kin, N. M., Meaney, M. J., \& Nair, N. P. (2002a). Acute modulation of aged human memory by pharmacol- 
ogical manipulation of glucocorticoids. Journal of Clinical Endocrinology and Metabolism, 87, 3798-3807.

Lupien, S. J., Wilkinson, C. W., Briere, S., Menard, C., Ng Ying Kin, N. M., \& Nair, N. P. (2002b). The modulatory effects of corticosteroids on cognition: Studies in young human populations. Psychoneuroendocrinology, 27, 401-416.

Lynn, S. J., \& Rhue, J. W. (1988). Fantasy proneness: Hypnosis, developmental antecedents, and psychopathology. American Psychologist, 43, 35-44.

Maheu, F. S., Collicutt, P., Kornik, R., Moszkowski, R., \& Lupien, S. J. (2005a). The perfect time to be stressed: A differential modulation of human memory by stress applied in the morning or in the afternoon. Progress in NeuroPsychopharmacology and Biological Psychiatry, 29, 1281-1288.

Maheu, F. S., Joober, R., \& Lupien, S. J. (2005b). Declarative memory after stress in humans: Differential involvement of the beta-adrenergic and corticosteroid systems. The Journal of Clinical Endocrinology and Metabolism, 90, 1697-1704.

Markowitsch, H. J., Kessler, J., Van der Ven, C., Weber-Luxenburger, G., Albers, M., \& Heiss, W-D. (1998). Psychic trauma causing grossly reduced brain metabolism and cognitive deterioration. Neuropsychologia, 36, 77-82.

Markowitsch, H. J., Kessler, J., Van der Ven, C., Weber-Luxenburger, G., Albers, M., \& Heiss, W-D. (2000). Neuroimaging and behavioral correlates of recovery from mnestic block syndrome and other cognitive deteriorations. Neuropsychiatry, Neuropsychology, and Behavioral Neurology, 13, 60-66.

Mather, M., Henkel, L. A., \& Johnson, M. K. (1997). Evaluating characteristics of false memories: Remember/know judgments and memory characteristics questionnaire compared. Memory \& Cognition, 25, 826-837.

Mauri, M., Sinforiani, E., Bono, G., Vignati, F., Berselli, M. E., Attanasio, R., et al. (1993). Memory impairment in Cushing's disease. Acta Neurologica Scandinavica, $87,52-55$.

Mazzoni, G. A. L. (2002). Naturally occurring and suggestion-dependent memory distortions: The convergence of disparate research traditions. European Psychologist, 7, 17-30.

Mazzoni, G. A. L., Loftus, E. F., \& Kirsch, I. (2001). Changing beliefs about implausible autobiographical events: A little plausibility goes a long way. Journal of Experimental Psychology: Applied, 7, 51-59.

Mazzoni, G. A. L., Loftus, E. F., Seitz, A., \& Lynn, S. J. (1999a). Changing beliefs and memories through dream interpretation. Applied Cognitive Psychology, 13, 125-144.

Mazzoni, G. A. L., Lombardo, P., Malvagia, S., \& Loftus, E. F. (1999b). Dream interpretation and false beliefs. Professional Psychology: Research and Practice, 30, 4550 .

Mazzoni, G. A. L., \& Kirsch, I. (2002). Autobiographical memories and beliefs: A preliminary metacognitive model. In T. J. Perfect \& B. L. Schwartz (Eds.), Applied metacognition (pp. 121-146). Cambridge: UK: Cambridge University Press. 
Mazzoni, G. A. L., \& Memon, A. (2003). Imagination can create false autobiographical memories. Psychological Science, 14, 186-188.

McDermott, K. B. (1996). The persistence of false memories in list recall. Journal of Memory and Language, 35, 212-230.

McEwen, B. S. (2002). Sex, stress, and the hippocampus: Allostasis, allostatic load and the aging process. Neurobiology of Aging, 23, 921-939.

McGaugh, J. L. (2000). Memory: A century of consolidation. Science, 287, 248-251.

McGaugh, J. L., \& Roozendaal, B. (2002). Role of adrenal stress hormones in forming lasting memories in the brain. Current Opinion in Neurobiology, 12, 205210 .

McGinnis, D., \& Roberts, P. (1996). Qualitative characteristics of vivid memories attributed to real and imagined experiences. American Journal of Psychology, 109, $59-77$.

McNair, D. M., Lorr, M., \& Droppleman, L. F. (1992). Profile Of Mood States (POMS) Manual. San Diego, CA: EdITS.

McNally, R. J. (2003). Remembering trauma. Cambridge, MA: Belknap Press/Harvard University Press.

McNally, R. J., Clancy, S. A., \& Schacter, D. L. (2001). Directed forgetting of trauma cues in adults reporting repressed or recovered memories of childhood sexual abuse. Journal of Abnormal Psychology, 110, 151-156.

McNally, R. J., Clancy, S. A., Schacter, D. L., \& Pitman, R. K. (2000). Personality profiles, dissociation, and absorption in women reporting repressed, recovered, or continuous memories of childhood sexual abuse. Journal of Consulting and Clinical Psychology, 68, 1033-1037.

Merckelbach, H. (2003). Taking recovered memories to court. In P. J. van Koppen (Ed.), Adversarial versus inquisitorial justice: Psychological perspectives on criminal justice systems. Perspectives in law \& psychology, Vol. 17 (pp. 119-130). New York: Kluwer Academic/Plenum Publishers.

Merckelbach, H., Dekkers, T., Wessel, I., \& Roefs, A. (2003a). Dissociative symptoms and amnesia in Dutch concentration camp survivors. Comprehensive Psychiatry, 44, 65-69.

Merckelbach, H., Dekkers, T., Wessel, I., \& Roefs, A. (2003b). Amnesia, flashbacks, nightmares, and dissociation in aging concentration camp survivors. Behaviour Research and Therapy, 41, 351-360.

Merckelbach, H., Horselenberg, R., \& Muris, P. (2001). The Creative Experience Questionnaire (CEQ): A brief self-report measure of fantasy proneness. Personality and Individual Differences, 31, 987-995.

Merckelbach, H., Smeets, T., Geraerts, E., Jelicic, M., Bouwen, A., \& Smeets, E. (2006). I haven't thought about this for years! Dating recent recalls of vivid memories. Applied Cognitive Psychology, 20, 33-42.

Miller, G. A. (1956). The magical number seven, plus or minus two: Some limits on our capacity for processing information. Psychological Review, 63, 81-97. 
Mitchell, K. J., Johnson, M. K., Raye, C. L., \& Greene, E. J. (2004). Prefrontal cortex activity associated with source monitoring in a working memory task. Journal of Cognitive Neuroscience, 16, 921-934.

Morgan, C. A., III, Hazlett, G., Doran, A., Garrett, S., Hoyt, G., Thomas, P., et al. (2004). Accuracy of eyewitness memory for persons encountered during exposure to highly intense stress. International Journal of Law and Psychiatry, 27, 265279.

Moritz, S., Gläscher, J., \& Brassen, S. (2005). Investigation of mood-congruent false and true memory recognition in depression. Depression and Anxiety, 21, 917.

Morris, C., Bransford, J. D., \& Franks, J. J. (1977). Levels of processing versus transfer appropriate processing. Journal of Verbal Learning and Verbal Behavior, 16, 519-533.

Moscovitch, M. (1992). Memory and working-with-memory: A component process model based on modules and central systems. Journal of Cognitive Neuroscience, 4, 257-267.

Moscovitch, M. (1994). Cognitive resources and dual-task interference effects at retrieval in normal people: The role of the frontal lobes and medial temporal cortex. Neuropsychology, 8, 524-534.

Moscovitch, M., \& Winocur, G. (1992). Frontal lobes and memory. In L. R. Squire (Ed.), The encyclopedia of learning and memory: A volume in neuropsychology (pp. 182187). New York: Macmillan.

Moscovitch, M., \& Winocur, G. (2002). The frontal cortex and working with memory. In D. T. Stuss \& R. T. Knight (Eds.), Principles of frontal lobe function (pp. 188-209). New York, NY: Oxford University Press.

Münsterberg, H. (1908). On the witness stand. New York: Doubleday.

Neisser, U. (1996). Remembering as doing. Behavioral and Brain Sciences, 19, 203-204.

Newcomer, J. W., Craft, S., Hershey, T., Askins, K., \& Bardgett, M. E. (1994). Glucocorticoid-induced impairment in declarative memory performance in adult humans. Journal of Neuroscience, 14, 2047-2053.

Newcomer, J. W., Selke, G., Melson, A. K., Hershey, T., Craft, S., Richards, K., \& Alderson, A. L. (1999). Decreased memory performance in healthy humans induced by stress-level cortisol treatment. Archives of General Psychiatry, 56, $527-$ 533.

Nicolson, N. A. (2004). Childhood parental loss and cortisol levels in adult men. Psychoneuroendocrinology, 29, 1012-1018.

Nicolson, N. A., \& van Diest, R. (2000). Salivary cortisol patterns in vital exhaustion. Journal of Psychosomatic Research, 49, 335-342.

Nieman, L., \& Cutler, G. B. (1995). Cushing's syndrome. In L. J. DeGroot, G. F. J. Cahill, L. Martini \& D. H. Nelson (Eds.), Endocrinology (Vol. 2, pp. 1741-1769). Philadelphia, PA.: Saunders. 
Nolde, S. F., Johnson, M. K., \& D’Esposito, M. (1998). Left prefrontal activation during episodic remembering: An event-related fMRI study. NeuroReport, 9, 3509-3514.

O’Brien, J. T. (1997). The "glucocorticoid cascade" hypothesis in man. Prolonged stress may cause permanent brain damage. British Journal of Psychiatry, 149, 191 201.

Ofshe, R., \& Watters, E. (1994). Making monsters: False memories, psychotherapy, and sexual bysteria. New York: Charles Scribner's Sons/MacMillan Publishing Co.

Oitzl, M. S., \& de Kloet, E. (1992). Selective corticosteroid antagonists modulate specific aspects of spatial orientation learning. Behavioral Neuroscience, 106, 6271.

Ost, J. (2003). Seeking the middle ground in the 'memory wars'. British Journal of Psychology, 94, 125-139.

Ost, J. (2006). Recovered memories. In T. Williamson (Ed.), Investigative interviewing: Rights, research, regulation (pp. 259-291). Devon, UK: Willan Publishing.

Ost, J., Costall, A., \& Bull, R. (2001). False confessions and false memories: A model for understanding retractors' experiences. The Journal of Forensic Psychiatry, 12, 549-579.

Ost, J., Costall, A., \& Bull, R. (2002a). A perfect symmetry? Retractors' experiences of recovering and retracting abuse memories. Psychology, Crime \& Law, 8, 155181.

Ost, J., Foster, S. Costall, A., \& Bull, R. (2005). False reports of childhood events in appropriate interviews. Memory, 13, 700-710.

Ost, J., Hogbin, I., \& Granhag, P. A. (2006). Altering false reports via confederate influence. Social Influence, 1, 105-116.

Ost, J., Vrij, A., Costall, A., \& Bull, R. (2002b). Crashing memories and reality monitoring: Distinguishing between perceptions, imaginations and 'false memories'. Applied Cognitive Psychology, 16, 125-134.

Otte, C., Hart, S., Neylan, T. C., Marmar, C. R., Yaffe, K., \& Mohr, D. C. (2005). A meta-analysis of cortisol response to challenge in human aging: Importance of gender. Psychoneuroendocrinology, 30, 80-91.

Paddock, J. R., Joseph, A. L., Chan, F. M., Terranova, S., Manning, C. G., \& Loftus, E. F. (1998). When guided visualization procedures may backfire: Imagination inflation and predicting individual differences in suggestibility. Applied Cognitive Psychology, 12, S63-S75.

Paddock, J. R., Noel, M., Terranova, S., Eber, H. W., Manning, C., \& Loftus, E. F. (1999). Imagination inflation and the perils of guided visualization. Journal of Psychology, 133, 581-595.

Parkin, A. J. (1997). The neuropsychology of false memory. Learning and Individual Differences, 9, 341-357. 
Parkin, A. J., Bindschaedler, C., Harsent, L. \& Metzler, C. (1996). Verification impairment in the generation of memory deficit following ruptured aneurysm of the anterior communicating artery. Brain and Cognition, 32, 14-27.

Parks, T. E. (1997). False memories of having said the unsaid: Some new demonstrations. Applied Cognitive Psychology, 11, 485-494.

Payne, D. G., Elie, C. J., Blackwell, J. M., \& Neuschatz, J. S. (1996). Memory illusions: Recalling, recognizing, and recollecting events that never occurred. Journal of Memory and Language, 35, 261-285.

Payne, J. D., Nadel, L., Allen, J. B., Thomas, K. G. F., \& Jacobs, W. J. (2002). The effects of experimentally induced stress on false recognition. Memory, 10, 1-6.

Pesant, N., \& Zadra, A. (2004). Working with dreams in therapy: What do we know and what should we do? Clinical Psychology Review, 24, 489-512.

Peters, M. J. V., Jelicic, M., Haas, N., \& Merckelbach, H. (2006). Mild executive dysfunctions in undergraduates are related to recollecting words never presented. International Journal of Neuroscience, 116, 1065-1077.

Peters, M. J. V., Jelicic, M., Verbeek, H., \& Merckelbach, H. (in press). Poor working memory predicts false memories. European Journal of Cognitive Psychology.

Pezdek, K., Finger, K., \& Hodge, D. (1997). Planting false childhood memories: The role of event plausibility. Psychological Science, 8, 437-441.

Pezdek, K., \& Lam, S. (in press). What research paradigms have cognitive psychologists used to study "False memory," and what are the implications of these choices? Consciousness and Cognition.

Platt, R. D., Lacey, S. C., Iobst, A. D., \& Finkelman, D. (1998). Absorption, dissociation, and fantasy-proneness as predictors of memory distortion in autobiographical and laboratory-generated memories. Applied Cognitive Psychology, 12, S77-S89.

Pope, H. G., Jr., Hudson, J. I., Bodkin, J., \& Oliva, P. (1998). Questionable validity of "dissociative amnesia" in trauma victims: Evidence from prospective studies. British Journal of Psychiatry, 172, 210-215.

Porter, S., Yuille, J. C., \& Lehman, D. R. (1999). The nature of real, implanted, and fabricated memories for emotional childhood events: Implications for the recovered memory debate. Law and Human Behavior, 23, 517-537.

Pruessner, J. C., Hellhammer, D. H., \& Kirschbaum, C. (1999). Burnout, perceived stress, and cortisol responses to awakening. Psychosomatic Medicine, 61, 197-204.

Pruessner, J. C., Kirschbaum, C., Meinlschmid, G., \& Hellhammer, D. H. (2003). Two formulas for computation of the area under the curve represent measures of total hormone concentration versus time-dependent change. Psychoneuroendocrinology, 28, 916-931.

Pruessner, J. C., Wolf, O. T., Hellhammer, D. H., Buske-Kirschbaum, A., von Auer, K., Jobst, S., et al. (1997). Free cortisol after awakening: A reliable biological marker for the assessment of adrenocortical activity. Life Sciences, 61, 25392549 . 
Rachman, S. (1993). Obsessions, responsibility, and guilt. Behaviour Research and Therapy, 31, 149-154.

Rachman, S. (2002). A cognitive theory of compulsive checking. Behaviour Research and Therapy, 40, 624-639.

Rachman, S., \& Shafran, R. (1999). Cognitive distortions: Thought-action fusion. Clinical Psychology and Psychotherapy, 6, 80-85.

Radley, J. J., Sisti, H. M., Hao, J., Rocher, A. B., McCall, T., Hof, P. R., et al. (2004). Chronic behavioral stress induces apical dendritic reorganizations in pyramidal neurons of the medial prefrontal cortex. Neuroscience, 125, 1-6.

Radomsky, A. S., Rachman, S., \& Hammond, D. (2001). Memory bias, confidence and responsibility in compulsive checking. Behaviour Research and Therapy, 39, 813-822.

Ranganath, C., Johnson, M. K., \& D’Esposito, M. (2000). Left anterior prefrontal activation increases with demands to recall specific perceptual information. Journal of Neuroscience, 20:RC108, 1-5.

Rassin, E., Merckelbach, H., \& Spaan, V. (2001). When dreams become a royal road to confusion: Realistic dreams, dissociation, and fantasy proneness. Journal of Nervous and Mental Disease, 189, 478-481.

Rassin, E., Merckelbach, H., Muris, P., \& Spaan, V. (1999). Thought-action fusion as a causal factor in the development of intrusions. Behaviour Research and Therapy, 37, 231-237.

Raye, C. L., Johnson, M. K., Mitchell, K. J., Nolde, S. F., \& D’Esposito, M. (2000). fMRI investigations of left and right PFC contributions to episodic remembering. Psychobiology, 28, 197-206.

Read, J. D. (1999). The recovered/false memory debate: Three steps forward, two steps back? Expert Evidence, 7, 1-24.

Read, J. D., \& Lindsay, D. S. (1994). Moving toward a middle ground on the "false memory debate": Reply to commentaries on Lindsay and Read. Applied Cognitive Psychology, 8, 407-435.

Redlich, A. D., Goodman, G. S. (2003). Taking responsibility for an act not committed: The influence of age and suggestibility. Law and Human Behavior, 27, 141-156.

Reul, J. M., \& de Kloet, E. R. (1985). Two receptor systems for corticosterone in rat brain: Microdistribution and differential occupation. Endocrinology, 117, 2505-2511.

Rey, A. (1964). L'examen clinique en psychologie. Paris : Presses Universitaires de France.

Reyna, V. F. (2000). Fuzzy-trace theory and source monitoring: An evaluation of theory and false-memory data. Learning and Individual Differences, 12, 163-175.

Reyna, V. F., \& Brainerd, C. J. (1995). Fuzzy-trace theory: An interim synthesis. Learning and Individual Differences, 7, 1-75. 
Riccio, D. C., Rabinowitz, V. C., \& Axelrod, S. (1994). Memory: When less is more. American Psychologist, 49, 917-926.

Riccio, D. C., Ackil, J. K., \& Burch-Vernon, A. (1992). Forgetting of stimulus attributes: Methodological implications for assessing associative phenomena. Psychological Bulletin, 112, 433-445.

Rimmele, U., Domes, G., Mathiak, K., \& Hautzinger, M. (2003). Cortisol has different effects on human memory for emotional and neutral stimuli. NeuroReport, 14, 2485-2488.

Roebers, C. M., \& Fernandez, O. (2005). The effects of accuracy motivation on children's and adults' event recall, suggestibility, and their answers to unanswerable questions. Journal of Cognition and Development, 3, 415-443.

Roediger, H. L., III (1996). Memory illusions. Journal of Memory and Language, 35, 300-318.

Roediger, H. L., III, \& Bergman, E. T. (1998). The controversy over recovered memories. Psychology, Public Policy, and Law, 4, 1091-1109.

Roediger, H. L., III, Jacoby, D., \& McDermott, K. B. (1996). Misinformation effects in recall: Creating false memories through repeated retrieval. Journal of Memory and Language, 35, 300-318.

Roediger, H. L., III, \& McDermott, K. B. (1995). Creating false memories: Remembering words not presented in lists. Journal of Experimental Psychology: Learning, Memory, and Cognition, 21, 803-814.

Roediger, H. L., III, \& McDermott, K. B. (2000). Tricks of memory. Current Directions in Psychological Science, 9, 123-127.

Roediger, H. L., III, Watson, J. M., McDermott, K. B., \& Gallo, D. A. (2001). Factors that determine false recall: A multiple regression analysis. Psychonomic Bulletin \& Review, 8, 385-407.

Roediger, H. L., III, Weldon, M. S., \& Challis, B. H. (1989). Explaining dissociations between implicit and explicit measures of retention: A processing account. In H. L., III Roediger \& F. I. M. Craik (Eds.), Varieties of memory and consciousness: Essays in honour of Endel Tulving (pp. 3-39). Hillsdale, NJ: Erlbaum.

Rohleder, N., Wolf, O. T., \& Kirschbaum, C. (2003). Glucocorticoid sensitivity in humans: Interindividual differences and acute stress effects. Stress, 6, 207-222.

Rohleder, N., Joksimovic, L., Wolf, J. M., \& Kirschbaum, C. (2004). Hypocortisolism and increased glucocorticoid sensitivity of pro-inflammatory cytokine production in Bosnian war refugees with posttraumatic stress disorder. Biological Psychiatry, 55, 745-751.

Rohrmann, S., Hennig, J., \& Netter, P. (1999). Changing psychobiological stress reactions by manipulating cognitive processes. International Journal of Psychophysiology, 33, 149-161.

Roozendaal, B. (2000). Glucocorticoids and the regulation of memory consolidation. Psychoneuroendocrinology, 25, 213-238. 
Roozendaal, B. (2002). Stress and memory: Opposing effects of glucocorticoids on memory consolidation and memory retrieval. Neurobiology of Learning and Memory, 78, 578-595.

Roozendaal, B., Hahn, E. L., Nathan, S. V., de Quervain, D. J., McGaugh, J. L. (2004). Glucocorticoid effects on memory retrieval require concurrent noradrenergic activity in the hippocampus and basolateral amygdala. Journal of Neuroscience, 24, 8161-8169.

Rosen, G. M. (Ed.). (2004). Posttraumatic stress disorder: Issues and controversies. New York: John Wiley \& Sons.

Ross, D. F., Ceci, S. J., Dunning, D., \& Toglia, M. P. (1994). Unconscious transference and mistaken identity: When a witness misidentifies a familiar but innocent person. Journal of Applied Psychology, 79, 918-930.

Rugg, M. D., Fletcher, P. C., Chua, P. M.-L., \& Dolan, R. J. (1999). The role of the prefrontal cortex in recognition memory and memory for source: An fMRI study. Neuroimage, 10, 520-529.

Sanchez, M. M., Young, L. J., Plotsky, P. M., \& Insel, T. R. (2000). Distribution of corticosteroid receptors in the rhesus brain: Relative absence of glucocorticoid receptors in the hippocampal formation. Journal of Neuroscience, 20, 4657-4668.

Sapolsky, R. M. (1996). Why stress is bad for your brain. Science, 273, 749-750.

Sapolsky, R. M., Krey, L. C., \& McEwen, B. S. (1985). Prolonged glucocorticoid exposure reduces hippocampal neuron number: Implications for aging. Journal of Neuroscience, 5, 1222-1227.

Sapolsky, R. M., \& Pulsinelli, W. A. (1985). Glucocorticoids potentiate ischemic injury to neurons: Therapeutic implications. Science, 229, 1397-1400.

Sapolsky, R. M., Romero, L. M., \& Munck, A. U. (2000). How do glucocorticoids influence stress responses? Integrating permissive, suppressive, stimulatory, and preparative actions. Endocrine Reviews, 21, 55-89.

Saufley, W. H., Jr., Otaka, S. R., \& Bavaresco, J. L. (1985). Context effects: Classroom tests and context independence. Memory \& Cognition, 13, 522-528.

Schacter, D. L. (1995). Memory distortion: How minds, brains and societies reconstruct the past. Cambridge, MA: Harvard University Press.

Schacter, D. L., Harbluk, J. L., \& McLachlan, D. (1984). Retrieval without recollection. Journal of Verbal Learning and Verbal Behavior, 23, 593-611.

Schacter, D. L., Koutstaal, W. \& Norman, K. A. (1997). False memories and aging. Trends in Cognitive Sciences, 1, 229-236.

Schacter, D. L., Reiman, E., Curran, T., Yun, L. S., Bandy, D., McDermott, K. B., \& Roediger, H. L., III. (1996). Neuroanatomical correlates of veridical and illusory recognition memory: Evidence from positron emission tomography. Neuron, 17, 267-274.

Schacter, D. L., \& Scarry, E. (2000). Memory, brain, and belief. Cambridge, MA: Harvard University Press. 
Schacter, D. L., \& Slotnick, S. D. (2004). The cognitive neuroscience of memory distortion. Neuron, 44, 149-160.

Scheck, B., Neufeld, P., \& Dwyer, J. (2001). Actual innocence: When justice goes wrong and how to make it right. New York: Signet Books.

Scheflin, A. W., \& Brown, D. (1996). Repressed memory or dissociative amnesia: What the science says. Journal of Psychiatry and Law, 24, 143-188.

Schelach, L., \& Nachson, I. (2001). Memory of Auschwitz survivors. Applied Cognitive Psychology, 15, 119-132.

Schommer, N. C., Hellhammer, D. H., \& Kirschbaum, C. (2003). Dissociation between reactivity of the hypothalamus-pituitary-adrenal axis and the sympathetic-adrenal-medullary system to repeated psychosocial stress. Psychosomatic Medicine, 65, 450-460.

Schooler, J. W. (1999). Seeking the core: The issues and evidence surrounding recovered accounts of sexual trauma. In L. M. Williams (Ed.), Trauma \& memory (pp. 203-216). Thousand Oaks, CA: Sage Publications, Inc.

Schooler, J. W., \& Loftus, E. F. (1986). Individual differences and experimentation: Complementary approaches to interrogative suggestibility. Social Behaviour, 1, 105-112.

Schwarz, N. (1999). Self-reports: How the questions shape the answers. American Psychologist, 54, 93-105.

Scoboria, A., Mazzoni, G., Kirsch, I., \& Relyea, M. (2004). Plausibility and belief in autobiographical memory. Applied Cognitive Psychology, 18, 791-807.

Scoboria, A., Mazzoni, G., Kirsch, I., \& Jimenez, S. (in press). The effects of prevalence and script information on plausibility, belief, and memory of autobiographical events. Applied Cognitive Psychology.

Scoville, W. B., \& Milner, B. (1957). Loss of recent memory after bilateral hippocampal lesions. Journal of Neurology, Neurosurgery, and Psychiatry, 20, 11-21.

Seeman, T. E., McEwen, B. S., Singer, B. H., Albert, M. S., \& Rowe, J. W. (1997). Increase in urinary cortisol excretion and memory declines: MacArthur studies of successful aging. Journal of Clinical Endocrinology and Metabolism, 82, 24582465.

Selye, H. (1936). Thymus and adrenals in the response of the organism to injuries and intoxications. British Journal of Experimental Pathology, 17, 234-248.

Selye, H. (1956). The stress of life. New York: McGraw-Hill.

Selye, H. (1973). The evolution of the stress concept. American Psychologist, 61, 692699.

Sephton, S. E., Sapolsky, R. M., Kraemer, H. C., \& Spiegel, D. (2000). Diurnal cortisol rhythm as a predictor of breast cancer survival. Journal of the National Cancer Institute, 92, 994-1000.

Shacham, S. (1983). A shortened version of the Profile of Mood States. Journal of Personality Assessment, 47, 305-306. 
Shafran, R., Thordarson, D. S., \& Rachman, S. (1996). Thought-action fusion in obsessive compulsive disorder. Journal of Anxiety Disorders, 10, 379-391.

Shalev, A. Y., Freedman, S., Peri, T., Brandes, D., Sahar, T., Orr, S. P., \& Pitman, R. K. (1998). Prospective study of posttraumatic stress disorder and depression following trauma. American Journal of Psychiatry, 155, 630-637.

Shors, T. J. (2006). Stressful experience and learning across the lifespan. Annual Review of Psychology, 57, 55-85.

Sivers, H., Schooler, J., \& Freyd, J. J. (2002). Recovered memories. Encyclopedia of the Human Brain, 4, 169-184.

Slotnick, S. D., Moo, L. R., Segal, J. B., \& Hart, J., Jr. (2003). Distinct prefrontal cortex activity associated with item memory and source memory for visual shapes. Cognitive Brain Research, 17, 75-82.

Smeets, T., Candel, I., \& Merckelbach, H. (2004). Accuracy, completeness, and consistency of emotional memories. American Journal of Psychology, 117, 595-609.

Smeets, T., Geraerts, E., Jelicic, M., \& Merckelbach, H. (in press). The awakening rise and diurnal pattern of cortisol and delayed recall of childhood sexual abuse memories. Psychiatry Research.

Smeets, T., Jelicic, M., \& Merckelbach, H. (2006a). Stress-induced cortisol responses, sex differences, and false recollections in a DRM paradigm. Biological Psychology, 72, 164-172.

Smeets, T., Jelicic, M., \& Merckelbach, H. (2006b). The effect of acute stress on memory depends on word valence. International Journal of Psychophysiology, 62, 3037.

Smeets, T., Jelicic, M., Merckelbach, H., Peters, M., Fett, A., Taverniers, J., Henquet, C., \& Dautzenberg, J. (in press). Enhanced memory performance on an internal-internal source monitoring test following acute psychosocial stress. Behavioral Neuroscience.

Smeets, T., Jelicic, M., Peters, M. J. V., Candel, I., Horselenberg, R., \& Merckelbach, H. (2006). "Of course I remember seeing that film!"-How ambiguous questions generate crashing memories. Applied Cognitive Psychology, 20, 779-789.

Smeets, T., Merckelbach, H., Horselenberg, R., \& Jelicic, M. (2005). Trying to recollect past events: Confidence, beliefs, and memories. Clinical Psychology Review, 25, 917-934.

Snodgrass, J. G., \& Corwin, J. (1988). Pragmatics of measuring recognition memory: Applications to dementia and amnesia. Journal of Experimental Psychology: General, 117, 34-50.

Spanos, N. P., Burgess, C. A., Burgess, M. F., Samuels, C., \& Blois, W. O. (1999). Creating false memories of infancy with hypnotic and non-hypnotic procedures. Applied Cognitive Psychology, 13, 201-218.

Spanos, N. P., Gwynn, M. I., Comer, S. L., Baltruweit, W. J., \& de Groh, M. (1989). Are hypnotically induced pseudomemories resistant to crossexamination? Law and Human Behavior, 13, 271-289. 
Spiro, R. J. (1980). Accommodative reconstruction in prose recall. Journal of Verbal Learning and Verbal Behavior, 19, 84-95.

Squire, L. R. (1992). Memory and the hippocampus: A synthesis from findings with rats, monkeys, and humans. Psychological Review, 99, 195-231.

Stadler, M. A., Roediger, H. L., III, \& McDermott, K. B. (1999). Norms for word lists that create false memories. Memory \& Cognition, 27, 494-500.

Stanford, S. C., \& Salmon, P. (1993). Stress: From synapse to syndrome. London: Academic Press.

Stanton, M. E., Gutierrez, Y. R., \& Levine, S. (1988). Maternal deprivation potentiates pituitary-adrenal stress responses in infant rats. Behavioral Neuroscience, 102, 692-700.

Starkman, M. N., Giordani, B., Berent, S., Schork, A., \& Schteingart, D. E. (2001). Elevated cortisol levels in Cushing's disease are associated with cognitive decrements. Psychosomatic Medicine, 63, 985-993.

Starkman, M. N., Giordani, B., Gebarski, S. S., Berent, S., Schork, M. A., \& Schteingart, D. E. (1999). Decrease in cortisol reverses human hippocampal atrophy following treatment of Cushing's disease. Biological Psychiatry, 46, 1595-1602.

Starkman, M. N., Giordani, B., Gebarski, S. S., \& Schteingart, D. E. (2003). Improvement in learning associated with increase in hippocampal formation volume. Biological Psychiatry, 53, 233-238.

Starkman, M. N., \& Schteingart, D. E. (1981). Neuropsychiatric manifestations of patients with Cushing's syndrome: Relationship to cortisol and adrenocorticotropic hormone levels. Archives of Internal Medicine, 141, 215-219.

Stefanis, N. C., Hanssen, M., Smirnis, N. K., Avramopoulos, D. A., Evdokimidis, I. K., Stefanis, C. N., et al. (2002). Evidence that three dimensions of psychosis have a distribution in the general population. Psychological Medicine, 32, 347-358.

Steptoe, A., Kunz-Ebrecht, S., Owen, N., Feldman, P. J., Willemsen, G., Kirschbaum, C., et al. (2003). Socioeconomic status and stress-related biological responses over the working day. Psychosomatic Medicine, 65, 461-470.

Sterling, P., \& Eyer, J. (1988). Allostasis: A new paradigm to explain arousal pathology. In S. Fisher \& J. Reason (Eds.): Handbook of life stress, cognition, and health (pp. 629-649). New York: John Wiley \& Sons, Ltd.

Sved, A. F., Cano, G., Passerin, A. M., \& Rabin, B. S. (2002). The locus coeruleus, Barrington's nucleus, and neural circuits of stress. Physiology and Behavior, 77, 737-742.

Takahashi, T., Ikeda, K., Ishikawa, M., Tsukasaki, T., Nakama, D., Tanida, S., \& Kameda, T. (2004). Social stress-induced cortisol elevation acutely impairs social memory in humans. Neuroscience Letters, 363, 125-130.

Thomas, A. K., \& Loftus, E. F. (2002). Creating bizarre false memories through imagination. Memory \& Cognition, 30, 423-431. 
Thomas, A. K., Bulevich, J. B., \& Loftus, E. F. (2003). Exploring the role of repetition and sensory elaboration in the imagination inflation effect. Memory $\& \mathrm{Cog}$ nition, 31, 630-640.

Thompson, L. A., Williams, K. L., L’Esperance, P. R., \& Cornelius, J. (2001). Context-dependent memory under stressful conditions: The case of skydiving. Human Factors, 43, 611-619.

Thomson, D. M., \& Tulving, E. (1970). Associative encoding and retrieval: Weak and strong cues. Journal of Experimental Psychology, 86, 255-262.

Tops, M., Van Der Pompe, G., Baas, D., Mulder, L. J. M., Den Boer, J. A., Meijman, T. F., et al. (2003). Acute cortisol effects on immediate free recall and recognition of nouns depend on stimulus valence. Psychophysiology, 40, 167-173.

Tousignant, J. P., Hall, D., \& Loftus, E. F. (1986). Discrepancy detection and vulnerability to misleading postevent information. Memory \& Cognition, 14, 329338.

Tulving, E. (1985). Memory and consciousness. Canadian Psychology, 26, 1-12.

Tulving, E. (2000). Concepts of memory. In E. Tulving \& F. I. M. (Eds.), The $O x$ ford handbook of memory (pp. 33-43). New York: Oxford University Press.

Tulving, E., \& Osler, S. (1968). Effectiveness of retrieval cues in memory for words. Journal of Experimental Psychology, 77, 593-601.

Tulving, E., \& Thomson, D. M. (1973). Encoding specificity and retrieval processes in episodic memory. Psychological Review, 80, 352-373.

Uno, H., Tarara, R., Else, J. G., Suleman, M. A., \& Sapolsky, R. M. (1989). Hippocampal damage associated with prolonged and fatal stress in primates. Journal of Neuroscience, 9, 1705-1711.

Van Cauter, E., \& Refetoff, S. (1985). Evidence for two subtypes of Cushing's disease based on the analyses of episodic cortisol secretion. New England Journal of Medicine, 312, 1343-1349.

Van der Kolk, B. A., \& Fisler, R. (1995). Dissociation and the fragmentary nature of traumatic memories: Overview and exploratory study. Joumal of Traumatic Stress, 8, 505-525.

Van Honk, J., Kessels, R. P. C., Putman, P., Jager, G., Koppeschaar, H. P. F., \& Postma, A. (2003). Attentionally modulated effects of cortisol and mood on memory for emotional faces in healthy young males. Psychoneuroendocrinology, 28, 941-948.

Van IJzendoorn, M. H., \& Schuengel, C. (1996). The measurement of dissociation in normal and clinical populations: Meta-analytic validation of the Dissociative Experiences Scale (DES). Clinical Psychology Review, 16, 365-382.

Van Koppen, P. J., \& Merckelbach, H. (1999). Characteristics of recovered memories: A Dutch replication of Gudjonsson's (1997) British survey. Applied Cognitive Psychology, 13, 485-489.

Van Oorsouw, K. I. M. (2006). I honestly can't remember. Dissociative amnesia as a metamemory phenomenon. Dissertation, Maastricht University, Maastricht. 
Van Stegeren, A. H., Everaerd, W., Cahill, L., McGaugh, J. L., \& Gooren, L. J. (1998). Memory for emotional events: Differential effects of centrally versus peripherally acting beta-blocking agents. Psychopharmacology, 138, 305-310.

Van Vreeswijk, M. F., \& De Wilde, E. J. (2004). Autobiographical memory specificity, psychopathology, depressed mood and the use of the Autobiographical Memory Test: A meta-analysis. Behaviour Research and Therapy, 42, 731-743.

Verhage, F. (1964). Intelligentie en leeftijd [Intelligence and age]. Assen: Van Gorcum.

Wade, K. A., Garry, M., Read, J. D., \& Lindsay, S. (2002). A picture is worth a thousand lies: Using false photographs to create false childhood memories. Psychonomic Bulletin \& Review, 9, 597-603.

Wade, K. A., Sharman, S. J., Garry, M., Memon, A., Mazzoni, G., Merckelbach, H., \& Loftus, E. F. (in press). False claims about false memory research. Consciousness and Cognition.

Wagenaar, W. A., \& Groeneweg, J. (1990). The memory of concentration camp survivors. Applied Cognitive Psychology, 4, 77-87.

Wald, F. D. M., \& Mellenbergh, G. J. (1990). De verkorte versie van de Nederlandse vertaling van de Profile of Mood States (POMS). [The shortened version of the Dutch translation of the Profile of Mood States (POMS)] Nederlands Tijdschrift voor de Psychologie [Dutch Journal of Psychology], 45, 86-90.

Wang, J., Rao, H., Wetmore, G. S., Furlan, P. M., Korczykowski, M., Dinges, D. F., et al. (2005). Perfusion functional MRI reveals cerebral blood flow pattern under psychological stress. Proceedings of the National Academy of Sciences of the United States of America, 102, 17804-17809.

Watanabe, Y., Gould, E., \& McEwen, B. S. (1992). Stress induces atrophy of apical dendrites of hippocampal CA3 pyramidal neurons. Brain Research, 588, 341345.

Wechsler, D. (1987). Wechsler Memory Scale-Revised manual. San Antonio: The Psychological Corporation.

Weissbecker, I., Floyd, A., Dedert, E., Salmon, P., \& Sephton, S. (2006). Childhood trauma and diurnal cortisol disruption in fibromyalgia syndrome. Psychoneuroendocrinology, 31, 312-324.

Wessa, M., Rohleder, N., Kirschbaum, C., \& Flor, H. (2006). Altered cortisol awakening response in posttraumatic stress disorder. Psychoneuroendocrinology, 31, 209-215.

Wessel, I., Merckelbach, H., \& Dekkers, T. (2002). Autobiographical memory specificity, intrusive memory, and general memory skills in Dutch-Indonesian survivors of World War II era. Journal of Traumatic Stress, 15, 227-234.

Wilson, K., \& French, C. C. (2006). The relationship between susceptibility to false memories, dissociativity, and paranormal belief and experience. Personality and Individual Differences, 41, 1493-1502. 
Wohlfarth, T. D., van den Brink, W., Winkel, F. W., \& ter Smitten, M. (2003). Screening for posttraumatic stress disorder: An evaluation of two self-report scales among crime victims. Psychological Assessment, 15, 101-109.

Wolf, O. T. (2003). HPA axis and memory. Best Practice \& Research Clinical Endocrinology and Metabolism, 17, 287-299.

Wolf, O. T., Convit, A., McHugh, P. F., Kandil, E., Thorn, E. L., De Santi, S., et al. (2001a). Cortisol differentially affects memory in young and elderly men. Behavioral Neuroscience, 115, 1002-1011.

Wolf, O. T., Fujiwara, E., Luwinski, G., Kirschbaum, C., \& Markowitsch, H. J. (2005). No morning cortisol response in patients with severe global amnesia. Psychoneuroendocrinology, 30, 101-105.

Wolf, O. T., Kudielka, B. M., Hellhammer, D. H., Hellhammer, J., \& Kirschbaum, C. (1998). Opposing effects of DHEA replacement in elderly subjects on declarative memory and attention after exposure to a laboratory stressor. Psychoneuroendocrinology, 23, 617-629.

Wolf, O. T., Kuhlmann, S., Buss, C., Hellhammer, D. H., \& Kirschbaum, C. (2004). Cortisol and memory retrieval in humans: Influence of emotional valence. Annals of the New York Academy of Sciences, 1032, 195-197.

Wolf, O. T., Schommer, N. C., Hellhammer, D. H., McEwen, B. S., \& Kirschbaum, C. (2001b). The relationship between stress induced cortisol levels and memory differs between men and women. Psychoneuroendocrinology, 26, 711720 .

Wolf, O. T., Schommer, N. C., Hellhammer, D. H., Reischies, F. M., \& Kirschbaum, C. (2002). Moderate psychosocial stress appears not to impair recall of words learned 4 weeks prior to stress exposure. Stress, 5, 59-64.

Wolkowitz, O. M., Reus, V. I., Weingartner, H., Thompson, K., Breier, A., Doran, A., Rubinow, D., \& Pickar, D. (1990). Cognitive effects of corticosteroids. American Journal of Psychiatry, 147, 1297-1303.

Wood, G. E., \& Shors, T. J. (1998). Stress facilitates classical conditioning in males, but impairs classical conditioning in females through activational effects of ovarian hormones. Proceeding of the National Academy of Sciences of the United States of America, 95, 4066-4071.

Wood, G. E., Beylin, A. V., \& Shors, T. J. (2001). The contribution of adrenal and reproductive hormones to the opposing effects of stress on trace conditioning in males versus females. Behavioral Neuroscience, 115, 175-187.

Woolley, C. S., Gould, E., McEwen, B. S. (1990). Exposure to excess glucocorticoids alters dendritic morphology of adult hippocampal pyramidal neurons. Brain Research, 531, 225-231.

Wüst, S., Federenko, I., Hellhammer, D. H., \& Kirschbaum, C. (2000a). Genetic factors, perceived chronic stress, and the free cortisol response to awakening. Psychoneuroendocrinology, 25, 707-720. 
Wüst, S., van Rossum, E. F., Federenko, I. S., Koper, J. W., Kumsta, R., \& Hellhammer, D. H. (2004). Common polymorphisms in the glucocorticoid receptor gene are associated with adrenocortical responses to psychosocial stress. Journal of Clinical Endocrinology and Metabolism, 89, 565-573.

Wüst, S., Wolf, J., Hellhammer, D. H., Federenko, I., Schommer, N., \& Kirschbaum, C. (2000b). The cortisol awakening response: Normal values and confounds. Noise and Health, 7, 77-85.

Yehuda, R. (1997). Stress and glucocorticoid. Science, 275, 1662-1663.

Yehuda, R. (2002). Post-traumatic stress disorder. New England Journal of Medicine, 346, 108-114.

Yehuda, R., Golier, J. A., \& Kaufman, S. (2005). Circadian rhythm of salivary cortisol in Holocaust survivors with and without PTSD. American Journal of Psychiatry, 162, 998-1000.

Yehuda, R., Kahana, B., Binder Brynes, K., Southwick, S. M., Mason, J. W., \& Giller, E. L. (1995). Low urinary cortisol excretion in Holocaust survivors with posttraumatic stress disorder. American Journal of Psycbiatry, 152, 982-986.

Yehuda, R., Southwick, S. M., Nussbaum, G., Wahby, V., Giller, E. L., Mason, J. W. (1990). Low urinary cortisol excretion in patients with posttraumatic stress disorder. Journal of Nervous Mental Disease, 178, 366-369.

Zoellner, L. A., Foa, E. B., Brigidi, B. D., \& Przeworski, A. (2000). Are trauma victims susceptible to "false memories?" Journal of Abnormal Psychology, 109, 517-524.

Zola-Morgan S. M., \& Squire, L. R. (1990). The primate hippocampal formation: Evidence for a time-limited role in memory storage. Science, 250, 288-290. 


\section{Summary}

Our memory is by no means infallible. In fact, there is a general consensus among experts about the reconstructive nature of episodic memory and its proneness to distortion and confabulation. That is, individuals may remember events or facts differently from how they originally occurred or may even remember events that did not take place at all. This consensus mainly stems from a vast body of research investigating whether people may come to falsely believe or remember fabricated information. Although these studies employed various research paradigms, like post-event or parental misinformation methods, most of them have merely shown participants' increased confidence that some fictitious childhood event might have happened to them, or have led them to falsely believe that some aversive event actually did happen in their childhood. Only a handful studies have really succeeded in implanting full-blown pseudo-memories.

Notwithstanding the vigorous pseudo-memory research from the past decade(s), it is not yet fully understood which factors may contribute to the development of false recollections. A recent study by Payne and co-workers (2002) showed that individuals who were exposed to acute stress displayed higher levels of pseudo-memories relative to non-stressed controls. Payne et al. conjectured that excessive levels of the primary human stress hormone cortisol were responsible for stressed participants' vulnerability to false recollections. The present dissertation further investigates the role of acute stress and cortisol elevations in the development of pseudo-memories, reviews episodic pseudo-memory studies that were carried out thus far and draws attention to their limitations, and examines whether the prominent rates at which people report to have seen non-existent images of highpublicity events can be accounted for by how people are probed for their knowledge of such footage.

Chapter 1 provides the reader with an overview of the history of the stress concept, explains the normal human stress response, considers whether long term stress exposure can cause irreversible brain damage, and evaluates the short term effects of glucocorticoids (i.e., primary stress hormones) on memory processes. Furthermore, Chapter 1 will briefly highlight the relationship between stress, amnesia, and pseudo-memories.

Quite a number of studies have demonstrated that acute stress can cause impairments in declarative memory performance, although this effect depends on the valence of the memory material. In Chapter 3, a study that further investigated the effects of acute stress on declarative memory performance as a function of stimulus valence is outlined. This study shows that stressed participants display impaired recall of neutral words, while their recall of positive emotional and negative emo- 
tional words remains unaffected. Participants who were stressed, however, generated more errors of commission than control participants.

Chapter 4 reports on two studies that attempted to replicate Payne and coworkers' (2002) findings showing that acute stress may lead people to falsely recognize non-presented words (i.e., critical lures). However, we were unable to replicate these findings in that stressed participants in both studies falsely recalled and recognized the non-presented critical lures at similar rates as non-stressed controls. The second experiment described in Chapter 4 also provides evidence that the magnitude of the cortisol responses cannot account for these non-significant findings. That is to say, this experiment shows that even participants who exhibit high cortisol responses do not demonstrate elevated levels of false recall or false recognition when compared to low cortisol responders or controls. Moreover, no significant correlations between false recall and recognition rates and delta increases in cortisol were detected.

Subsequently, Chapter 5 explores whether acute stress or the ensuing cortisol elevations can influence source monitoring performance (i.e., the ability to make attributions about the origins of memories, knowledge and beliefs). This study indicates that relative to controls, participants exposed to the stressor exhibited better correct identification of the source of their memories than non-stressed controls. Furthermore, relative to controls, stressed participants were more accurate at discriminating targets from distracters. This implies that exposure to acute stress may render people less likely to confuse the source of their memory.

Chapter 6 empirically tests the hypothesis that neuroendocrine alterations of the hypothalamic-pituitary-adrenal axis may explain delayed recall of childhood sexual abuse (CSA). Morning cortisol responses and diurnal cortisol patterns of women reporting continuous, repressed, or recovered memories of CSA were compared to non-abused control participants. Neither the awakening rise nor the diurnal pattern of cortisol differed between CSA groups and controls, with all four groups showing a straightforward morning response and a typical diurnal rhythm of cortisol secretion. Thus, contrary to what one would predict on the basis of certain popular theories from the recovered memory literature, this chapter provides tentative evidence that there are no abnormalities in cortisol patterns of women who have repressed, recovered, or continuous CSA memories.

An ever growing body of literature maintains to examine pseudo-memories. Chapter 7 argues that not all research paradigms that have been employed over the years have succeeded in implanting full-blown pseudo-memories. It is asserted that examination of behavioural consequences of the memory manipulations is crucial to progress in research on pseudo-memories.

Chapter 7 also addresses the so-called crashing memory paradigm in which individuals claim to have seen non-existent footage of high-publicity events. Chapter 8 explores whether the ambiguity and/or specificity of the critical crashing memory question may help explain the high rates at which people tend to endorse these 
false reports. This study makes clear that while 38 percent of participants overall erroneously reported having seen non-existent footage of the Pim Fortuyn assassination, the number of participants falsely reporting to have seen the non-existent video footage was greatly reduced by explicitly mentioning the boundaries of the critical incident for which they were probed.

In conclusion, the General Discussion summarizes the current dissertation's main findings and suggests some ideas that might prove fruitful for future research to pursue. 


\section{Samenvatting}

Ons geheugen is allesbehalve perfect. Onder geheugenexperts bestaat consensus over het reconstructief te werk gaan van het menselijke geheugen dat daardoor vatbaar is voor allerhande distorties en confabulaties. Met andere woorden, mensen herinneren zich gebeurtenissen soms anders dan hoe ze werkelijk plaats vonden of herinneren zich zelfs gebeurtenissen die in werkelijkheid helemaal niet plaats vonden. Deze consensus is voornamelijk te danken aan een scala aan studies die onderzochten of mensen ten onrechte kunnen gaan geloven in fictieve gebeurtenissen en of zij daar zelfs herinneringen aan gaan vormen. Hoewel deze studies verscheidene onderzoeksparadigma's gebruikten, zoals het presenteren van misleidende informatie, zou men kunnen stellen dat het merendeel van deze studies enkel aangetoond heeft hoe mensen gebeurtenissen waarschijnlijker kunnen gaan vinden na bepaalde geheugenmanipulaties. Slechts een klein aantal studies gaat een stap verder en toont aan dat mensen ook daadwerkelijk kunnen gaan geloven in de hen gesuggereerde misleidende informatie. Maar een paar studies zijn erin geslaagd individuen volledige, gedetailleerde pseudo-herinneringen te laten vormen.

Niettegenstaande het vele onderzoek van de afgelopen decennia, is men er nog niet helemaal uit welke factoren kunnen bijdragen aan het ontstaan van pseudo-herinneringen. Een studie van Payne en haar collegae (2002) toonde onlangs aan dat proefpersonen na blootstelling aan een acute stressor meer pseudoherinneringen ontwikkelden ten opzichte van een (niet gestresste) controlegroep. Payne e.a. veronderstelden dat excessief verhoogde niveaus van cortisol (het primaire menselijke stresshormoon) verantwoordelijk waren voor de door hen gevonden resultaten. Het huidige proefschrift rapporteert over verder onderzoek naar de relatie tussen acute stress en cortisol enerzijds, en het ontstaan van pseudoherinneringen anderzijds. Verder zal dit proefschrift een overzicht bieden van het onderzoek dat totnogtoe is verricht naar pseudo-herinneringen, en de beperkingen ervan bloot leggen. Ook zal er onderzocht worden waarom zoveel mensen in voorgaande onderzoeken aangaven zich beelden te herinneren van prominente, hoog emotionele gebeurtenissen, die in feite helemaal niet bestaan.

Hoofdstuk 1 beschrijft kort de geschiedenis van het concept "stress" en de normale menselijke stress respons, staat stil bij de vraag of chronische stress onherroepelijke schade aan de hersenen kan toebrengen, en omschrijft de mogelijke effecten op het (declaratieve) geheugen van kortstondige stress en glucocorticoïden. Bovendien zal dit hoofdstuk kort de relatie tussen stress, amnesie, en pseudoherinneringen bespreken.

Hoewel het effect mede afhankelijk is van de valentie van het stimulusmateriaal, hebben talloze onderzoeken aangetoond dat acute stress geheugenondermij- 
nend kan werken. Hoofdstuk 3 beschrijft een studie die de interactie tussen stimulus valentie en de effecten van acute stress verder onderzoekt. Deze studie toont aan dat acute stress leidt tot slechtere prestaties op een geheugentaak als het gaat om neutrale woorden, maar dat emotioneel positieve en emotioneel negatieve woorden relatief immuun zijn voor dit effect. Ook werd er door proefpersonen die vooraf gestresst werden meer commissie fouten gemaakt ten opzichte van de niet gestresste controle proefpersonen.

Hoofdstuk 4 beschrijft twee studies waarin werd geprobeerd de resultaten van Payne en collegae (2002), die aantoonden dat acute stress tot meer pseudoherinneringen kan leiden, te repliceren. Beide studies slaagden daar niet in: het aantal pseudo-herinneringen verschilde niet tussen de stress en de controle groepen. De tweede studie laat bovendien zien dat er geen verschillen zijn tussen mensen die sterke cortisol stijgingen vertonen en mensen met minder sterke reacties, waardoor verschillen in cortisol reacties maar moeilijk verantwoordelijk geacht kunnen worden voor deze nulbevindingen. Overigens, er werden geen significante correlaties ontdekt tussen het aantal pseudo-herinneringen en individuele cortisol reacties.

Vervolgens onderzocht de studie in Hoofdstuk 5 of acute stress invloed heeft op het aantal bronverwarringsfouten (dwz., fouten in het toeschrijven van een bron aan bepaalde herinneringen, kennis, of opvattingen). Deze studie laat zien dat ten opzichte van niet gestresste controle proefpersonen, de gestresste proefpersonen beter in staat zijn om de bron van hun herinneringen in kaart te brengen. Ook waren zij beter in het onderscheiden van de echte stimuli van afleidende stimuli. Deze resultaten impliceren dat in stressvolle situaties, mensen beter in staat zijn om de bron van hun herinnering te achterhalen.

In Hoofdstuk 6 vindt de lezer een empirische toetsing van de hypothese dat hervonden herinneringen - traumatische herinneringen waarvan diegenen die ze ervaren zeggen dat ze gedurende enige tijd verdrongen of ontoegankelijk voor hen waren - verklaard kunnen worden door neuroendocrine veranderingen in de werking van de hypothalamus-hypofyse-bijnier as. Hiertoe werden de ochtend reacties en het circadiane ritme van cortisol secretie gemeten onder mensen die claimen traumatische herinneringen te hebben hervonden of verdrongen, mensen die altijd hebben geweten een traumatische gebeurtenis te hebben meegemaakt in de kindertijd, en niet getraumatiseerde controle proefpersonen. Noch de ochtend respons, noch het circadiane ritme van cortisol afscheiding verschilde tussen deze groepen, waarbij alle groepen een normale cortisol stijging in de ochtend en continu afnemende waarden over de dag vertoonden. In tegenstelling tot wat wel eens in de populaire literatuur over hervonden herinneringen wordt geventileerd, levert deze studie geen enkele ondersteuning voor het idee dat neuroendocrine veranderingen in de hypothalamus-hypofyse-bijnier as werking verantwoordelijk kunnen zijn voor het verdringen en later weer terugvinden van traumatische herinneringen.

Nogal wat experimentele studies beweren volledige, autobiografische pseudoherinneringen te hebben onderzocht, dan wel hierover harde uitspraken te kunnen 
doen. Hoofdstuk 7 beargumenteert waarom niet al deze studies hierover harde uitspraken kunnen en mogen doen. De in zulke studies gehanteerde methodologie laat dat immers niet toe. Verder wordt in Hoofdstuk 7 geopperd dat indien onderzoek naar pseudo-herinneringen vooruitgang wil blijven boeken en nauwer wil aansluiten bij praktisch relevante kwesties (bv., fictieve herinneringen an seksueel misbruik), er in het laboratorium ook moet worden gekeken naar de aanwezigheid van gedragsconsequenties van de toegepaste geheugenmanipulaties.

Hoofdstuk 7 bespreekt eveneens studies die van het zogenaamde "crashing memory" paradigma gebruik maken. In dergelijk onderzoek wordt proefpersonen gevraagd of ze zich een bepaald videofragment van een hoog emotionele, publieke gebeurtenis kunnen herinneren, dat echter in werkelijkheid helemaal niet bestaat. Veelal geeft het merendeel van proefpersonen aan het niet-bestaande filmfragment te hebben gezien. In Hoofdstuk 8 wordt onderzocht of de ambiguiteit en/of de specificiteit van de vraagstelling gedeeltelijk zou kunnen verklaren waarom zoveel mensen beweren niet-bestaande videofragmenten te hebben gezien. De resultaten tonen aan dat hoewel 38 procent van alle ondervraagden beweert (niet-bestaande) videobeelden te hebben gezien van de moord op Pim Fortuyn, het aantal mensen dat deze bewering is toegedaan mede afhangt van hoe precies men de vraag omschrijft.

Tot slot zal de Algemene Discussie een korte synthese van de onderzoeksresultaten van dit proefschrift weergeven, en enkele ideeën voor vervolgonderzoek beschrijven. 


\section{Dankwoord}

Onderzoek doe je niet alleen, maar is het resultaat van velen. De voorbije jaren hebben dan ook heel wat mensen geholpen bij het tot stand komen van dit proefschrift. Ik wil dan ook iedereen van harte dank heten, en enkelen wil ik daarbij expliciet vernoemen (diegene die ik bij vergetelheid niet noem: mijn oprechte excuses).

Beste Harald en Marko, promotor en co-promotor, bedankt. Harald, je staat bekend als een erudiete wetenschapper, een autoriteit in zijn vakgebied met een uitzonderlijke kennis van de vakliteratuur. Dat kan ik volmondig bevestigen. Daarnaast ben je ook nog een uitstekende promotor die steeds weer tijd maakte om manuscripten na te kijken en te voorzien van buitengewoon grondig commentaar, waaruit ik heel veel kon leren. Harald, ik dank jou voor alle tijd en moeite die je in mij stopte en omdat je mij de mogelijkheid gaf om te assisteren met enkele merkwaardige dossiers. Marko, steeds was je onmiddellijk bereikbaar en bereid om over onderzoeksvoorstellen, interessante congressen, budgettering, maar ook zaken uit de media of andere interessante wetenswaardigheden van gedachten te wisselen. Keer op keer maakte je tijd voor mijn vragen en de manuscripten die ik je voorlegde kwamen altijd spoedig retour voorzien van gepast commentaar. Marko, bedankt voor je geduld en de voortreffelijke begeleiding die ik van je mocht ontvangen. Harald en Marko, ik heb jullie spontane, verhelderende inbreng bij het bedenken van nieuwe experimenten erg gewaardeerd. Ik heb erg veel van jullie beiden geleerd en verheug me op onze toekomstige samenwerking. Ik prijs mezelf erg gelukkig dat ik jullie als begeleiders mocht hebben. Daarbij weet ik jullie beiden niet alleen als wetenschapper te waarderen, maar ook als mens.

Graag wil ik alle collega's van de vakgroep Experimentele Psychologie bedanken voor het stimulerend onderzoeksklimaat. Ook het instituut EPP dank ik hiervoor. Bovenal wil ik mijn directe collega's van de Interfacultaire werkgroep RechtsPsychologie (IRP) bedanken voor de goede samenwerking, de altijd interessante bijeenkomsten, de geanimeerde discussies, de gezellige etentjes, en de erg leuke jaarlijkse retraite. Dus bedankt: Beatrijs, Corine, Elke, Ewout, Han, Hans, Harald, Henk, Henry, Ingrid, Kim, Maaike, Maarten, Marko, Mirthe, Peter, Robert, Saskia, Timo, en Wilma. Ook dank aan de ex-IRP'ers Ineke en Sander. Extra dank gaat uit naar Kim voor het steeds weer feilloos organiseren van alle IRPactiviteiten.

Ingrid, ook voor jou een speciaal woord van dank: Jij was het die me als student enthousiasmeerde voor het onderzoek, me de eerste kneepjes van het vak leerde, en zo mijn verdere carrière heeft beïnvloedt. Anita, ook jij bedankt omdat je 
me de kans gaf me als jouw student-assistent verder te bekwamen in het onderzoek.

Beste Maarten en Christophe, paranimfen. Bedankt om tijdens de promotie samen met mij op het podium te willen staan, en in het bijzonder voor jullie vriendschap en steun. Maarten, dank je voor de aangename werksfeer en de gesprekken die we over alles en nog wat voerden.

Naast mijn directe collega's hebben ook andere UM-ers een bijdrage geleverd aan het huidige proefschrift. Ik denk daarbij aan alle studenten die de afgelopen jaren met het verzamelen en invoeren van alle data hebben geholpen. Ik dank jullie voor de inzet en volharding die nodig was bij het werven en testen van alle proefpersonen. Ook wil ik van de gelegenheid gebruik maken om José Sulon te bedanken voor het analyseren van alle cortisol monsters. Nancy Nicolson wil ik bedanken voor haar hulp met de DAC-analyses (hoofdstuk 6) en haar uitvoerig commentaar op een eerdere versie van bijhorend manuscript.

Tot slot wil ik enkele mensen bedanken die me te allen tijde hebben aangemoedigd bij deze uitdaging. Pa en ma, bedankt dat jullie mij de kans hebben gegeven om verder te studeren en me gesteund hebben in alles wat ik wilde bereiken. Schoonouders en naaste familie, bedankt voor jullie steun en vertrouwen in een goede afloop. Christophe, bedankt voor je relativerende opmerkingen, de soms diep filosofische en soms lichtzinnige maar steeds vermakelijke gesprekken, de gezellige avonden en etentjes, en omdat je de beste vriend bent die ik me wensen kan. Last but not least: Liefste Linda, je onvoorwaardelijke steun hielp me in goede én minder goede tijden. Zonder jou was het nooit gelukt. Dank je wel, voor alles, maar in het bijzonder datgene wat geen betrekking heeft op dit proefschrift. 


\section{Curriculum vitae}

Tom Smeets was born in Heusden-Zolder (Belgium) on April 13th 1978. He started his study in Psychology at Maastricht University in September 1999 and graduated cum laude the 31st of May 2003. In June 2003, he commenced his Ph-D research at the Department of Experimental Psychology, Maastricht University, where he has conducted several studies on the effect of acute (psychosocial) stress and cortisol on memory. He currently holds a position as Postdoctoral Fellow at the same department, where he continues his research on stress and memory.

Tom Smeets werd op 13 april 1978 geboren te Heusden-Zolder (België). In september 1999 startte hij met de studie psychologie te Maastricht, die hij in mei 2003 cum laude afrondde. In juni 2003 werd hij als Assistent In Opleiding aangesteld bij de vakgroep Experimentele Psychologie van de Universiteit Maastricht, waar hij onderzoek deed naar de effecten van stress en cortisol op het geheugen. Momenteel is hij bij diezelfde vakgroep werkzaam als Post-doc onderzoeker, alwaar hij verder onderzoek blijft verrichten naar stress en het geheugen. 


\section{List of publications}

\section{International journal articles}

Cima, M., Smeets, T., \& Jelicic, M. (submitted for publication). Self-reported trauma, cortisol levels, and aggression in psychopathic and non-psychopathic prison inmates.

Giesbrecht, T., Smeets, T., \& Merckelbach, H. (submitted for publication). Dissociative experiences on ice. Peritraumatic and trait dissociation during the cold pressor test.

Giesbrecht, T., Smeets, T., Merckelbach, H., Jelicic, M., \& Leppink, J. (submitted for publication). Acute dissociation after one night of sleep loss.

Giesbrecht, T., Smeets, T., Merckelbach, H., \& Jelicic, M. (in press). Depersonalization experiences in undergraduates are related to heightened stress cortisol responses. The Journal of Nervous and Mental Disease.

Horselenberg, R., Merckelbach, H., Smeets, T., Franssens, D., Peters, G. J. Y., \& Zeles, G. (2006). False confessions in the lab: Do plausibility and consequences matter? Psychology, Crime \& Law, 12, 61-75.

Jansen, A., Smeets, T., Boon, B., Nederkoorn, C., \& Mulkens, S. (in press). Vulnerability to interpretation bias in overweight children. Psychology \& Health.

Jansen, A., Smeets, T., \& Nederkoorn, C. (2006). I see what you see: The lack of a self-serving body image bias in eating disorders. British Journal of Clinical Psychology, 45, 123-135.

Jelicic, M., Smeets, T., Peters, M. J. V., Candel, I., Horselenberg, R., \& Merckelbach, H. (2006). Assassination of a controversial politician: Remembering details from another non-existent film. Applied Cognitive Psychology, 20, 591-596.

Merckelbach, H., Jelicic, M., Smeets, T., \& Merten, T. (submitted for publication). Tod auf dem Bahnübergang oder Wie eine geltend gemachte Amnesie forensisch begutachtet wird.

Merckelbach, H., Peters, M., Jelicic, M., Brands, I., \& Smeets, T. (2006). Detecting malingering of Ganser-like symptoms with tests: A case study. Psychiatry and Clinical Neurosciences, 60, 636-638.

Merckelbach, H., Smeets, T., Geraerts, E., Jelicic, M., Bouwen, A., \& Smeets, E. (2006). I haven't thought about this for years! Dating recent recalls of vivid memories. Applied Cognitive Psychology, 20, 33-42. 
Peters, M., Cima, M., Smeets, T., Jelicic, M., \& Merckelbach, H. (submitted for publication). Did I say that word or did you? Executive dysfunctions and susceptibility to leading questions influence memory functioning but not source attribution in schizophrenia.

Peters, M., Smeets, T., Giesbrecht, T., Jelicic, M., \& Merckelbach, H. (submitted for publication). Confusing action and imagination: Action source monitoring in schizotypy.

Smeets, T., Candel, I., \& Merckelbach, H. (2004). Accuracy, completeness, and consistency of emotional memories. American Journal of Psychology, 117, 595-609.

Smeets, T., Geraerts, E., Jelicic, M., \& Merckelbach, H. (in press). The awakening rise and diurnal pattern of cortisol and delayed recall of childhood sexual abuse memories. Psychiatry Research.

Smeets, T., Jelicic, M., \& Merckelbach, H. (2006). Stress-induced cortisol responses, sex differences, and false recollections in a DRM-paradigm. Biological Psychology, 72, 164-172.

Smeets, T., Jelicic, M., \& Merckelbach, H. (2006). The effect of acute stress on memory depends on word valence. International Journal of Psychophysiology, 62, 3037.

Smeets, T., Jelicic, M., Merckelbach, H., Peters, M., Fett, A., Taverniers, J., Henquet, C., \& Dautzenberg, J. (in press). Enhanced memory performance on an internal-internal source monitoring task following acute psychosocial stress. Behavioral Neuroscience.

Smeets, T., Jelicic, M., Peters, M.J.V., Candel, I., Horselenberg, R., \& Merckelbach, H. (2006). "Of course I remember seeing that film!'-How ambiguous questions generate crashing memories. Applied Cognitive Psychology, 20, 779-789.

Smeets, T., Leppink, J., Jelicic, M., \& Merckelbach, H. (submitted for publication). Shortened versions of the Gudjonsson Suggestibility Scale meet the standards.

Smeets, T., Merckelbach, H., Horselenberg, R., \& Jelicic, M. (2005). Trying to recollect past events: Confidence, beliefs, and memories. Clinical Psychology Review, $25,917-934$.

\section{National journal articles}

Horselenberg, R., \& Smeets, T. (2005). Valse bekentenissen. Advocatenblad, 14, 620624.

Jansen, A., Nederkoorn, C., Smeets, T., Havermans, R., \& Martijn, C. (2006). Jij ziet, jij ziet, wat ik niet zie: Het vertekende lichaamsbeeld van gezonde vrouwen. De Psycholoog, 41, 518-526.

Merckelbach, H., Peters, M., Jelicic, M., Brands, I., \& Smeets, T. (2006). Hoe meneer Ganser zijn basketbalteam naar de overwinning voerde. Maandblad Geestelijke Volksgezondheid, 61, 614-627. 
Merckelbach, H., Smeets, T., Peters, M., \& Jelicic, M. (2005). Op weg naar een valse bekentenis. Een schizofrene patiënt wordt verhoord. Maandblad Geestelijke Volkesgezondheid, 60, 827-243.

Smeets, T. (2004). Waarom bekentenissen niet als enige bewijsmiddel mogen gelden. Skepter, 17, 18-21.

Smeets, T., Merckelbach, H., Horselenberg, R., \& Jelicic, M. (2004). Vroeger met je hand door een ruit gevallen: Schatting, geloof, of herinnering? De Psycholoog, 39, 485-491.

\section{Book contributions and various}

Jelicic, M., Merckelbach, H., \& Smeets, T. (2005). Industrie en onderzoek. Reactie op G. C. G. Dehue, 'Verdienen aan neerslachtigheid' (MGv-05-3). Maandblad Geestelijke Volksgezondheid, 60, 372-373.

Jelicic, M., Smeets, T., Candel, I., van Suijdam, M., \& Merckelbach, H. (2006). No, I don't remember seeing video footage of the killing of Theo van Gogh! Misinformation manipulations do not always elicit false memories. In: D. Canter (Series Ed.) \& K. Nixon (Vol. Ed.), Forensic recall and eyewitness testimony (pp. 21 25). London: IA-IP Publishing.

Smeets, T. (2006). Getuige-deskundigen in strafzaken. Maandblad Geestelijke Volksgezondheid, 61, 453-455.

Smeets, T., Jelicic, M., \& Merckelbach, H. (2006). Reduced hippocampal and amygdalar volume in DID: Not such clear evidence [Letter to the Editor]. American Journal of Psychiatry, 163, 1643.

Smeets, T., \& van Koppen, P.J. (2004). Ontdekken en herontdekken van het verleden. In K. Hendriks (Ed.), V aag verleden. Hoe ik ging geloven in fictieve herinneringen. Amsterdam: L.J. Veen. 


\section{Stressing Memories}

Acute Stress, Cortisol, and Psendo-memories

\section{Tom Smeets}

Today, there is a broad consensus among cognitive psychologists about the reconstructive nature of episodic memory and its proneness to distortion and confabulation. Despite the vigorous research of the past decades, researchers are still searching for factors that contribute to the formation of pseudo-memories, or which type of person is particularly vulnerable to these false recollections. The aim of the present dissertation is to investigate whether acute stress and/or the consequential cortisol (i.e., the primary human stress hormone) elevations might foster the development of pseudo-memories. A secondary aim of this dissertation is to take a closer look at what type of pseudo-memory research has been carried out to date, highlight some of the limitations that are inherent in the current research paradigms, and suggest some new directions. The thesis discusses how the current findings relate to contemporary work and suggests some directions for future research. 